\title{
Cognitive Flexibility in the context of Stress and Depressive Disorders
}

\author{
Robert Lukasz Gabrys
}

\section{THESIS}

Submitted to the Faculty of Graduate and Postdoctoral Affairs in Partial Fulfillment of the requirements for the degree of

Doctor of Philosophy

in

Neuroscience

\author{
Carleton University \\ Ottawa, Canada \\ (C)2017 \\ Robert Lukasz Gabrys
}




\begin{abstract}
Disturbances of cognitive flexibility may contribute to difficulties in emotion regulation, which can lead to the emergence of depressive illness. However, there are limited data available as to how stressful experiences influence cognitive flexibility. The purpose of the present research was to examine the contribution of different stressor experiences, including selfreported early-life trauma, on cognitive flexibility, and how disturbances in this regard were related to heightened depressive symptoms and to stress-reactivity. Given that the neutrophin brain-derived neurotrophic factor (BDNF) has been linked to mood, notably depression, the link between a BDNF polymorphism and cognitive flexibility was also examined. In Study 1 ( $N=$ 64), an acute psychosocial stressor (the Trier Social Stress Test; TSST) was accompanied by enhanced cognitive flexibility, as reflected through greater set-shifting performance on the Wisconsin Card Sorting Task (WCST), and this relationship was mediated by heightened threat appraisal. However, the enhanced set-shifting performance was not apparent among individuals with elevated depressive symptoms. Study $2(N=239)$ demonstrated that, among Met allele carriers for the BDNF Val66Met gene polymorphism, greater frequency of traumatic events was associated with decreased cognitive flexibility (i.e., reduced set-shifting performance of the WCST), and these relations depended on type of trauma experienced and gender. In Studies 3A, $\mathrm{B}$, and $\mathrm{C}$, a cognitive flexibility questionnaire (CFQ) was developed which identified the ways in which cognitive flexibility might be manifested in stressful situations. The CFQ, which comprised two sub-scales (cognitive control and cognitive resources), exhibited high internal consistency, and was associated with depressive symptoms even after controlling for other measures that have been linked to depression. Finally, in Study $4(N=44)$, it was demonstrated that reduced cognitive flexibility, as assessed by the CFQ, was associated with negative affect
\end{abstract}


and cortisol levels following the TSST. Collectively, the present findings indicate that the impact of stressful events on cognitive flexibility depends on the nature of the stressor as well as personal characteristics, including gender and genetic disposition. Moreover, the present research further supports the notion that disturbances in fundamental cognitive control processes, such as cognitive flexibility, might contribute to the maintenance of negative emotional states and neuroendocrine activation, and might contribute to the evolution of depressive illness. 


\section{Acknowledgements}

First and foremost, I would like to extend a great deal of gratitude to my mentor, Dr. Hymie Anisman, for all of the knowledge, encouragement, and support he has provided over the course of my graduate studies. I have learned many things, both professionally and personally, from Dr. Anisman, and for that I will always be grateful. I would also like to thank my thesis committee members, Dr. Kim Hellemans and Dr. Alfonso Abizaid, for their expertise and guidance throughout this entire research endeavour. My friends and colleagues, especially Robyn McQuaid, Opal McInnis, Jesse Howell, Christopher Rudyk, and Niko Tzakis, you deserve a great deal of appreciation for your support and for making graduate school a fun and exciting

time. As well, I would like to thank Diane Trenouth as well as all the staff, faculty, and students in the Department of Neuroscience that have created a positive, stimulating, and encouraging environment to work in throughout the years. Finally, and most importantly, I would like to thank my parents and Adrian for their unconditional and unwavering support from beginning to end. I am forever indebted to you. 


\section{Table of Contents}

Title

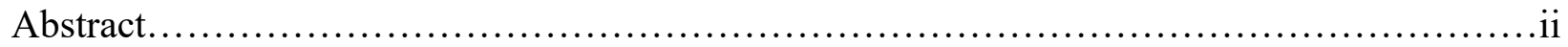

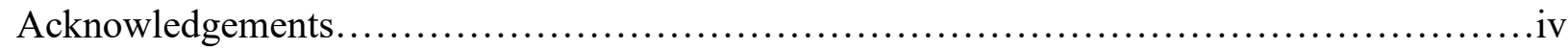

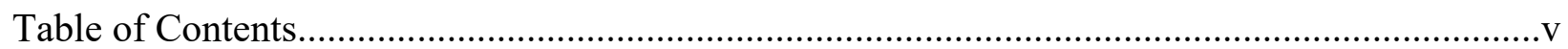

List of Tables.................................................................................................................

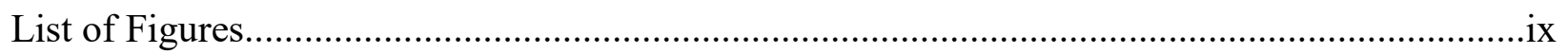

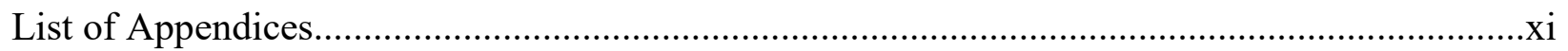

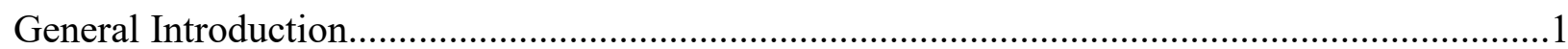

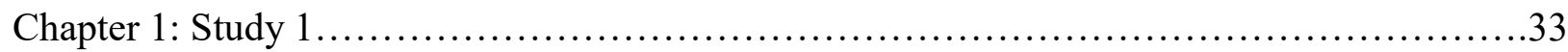

Abstract......................................................................

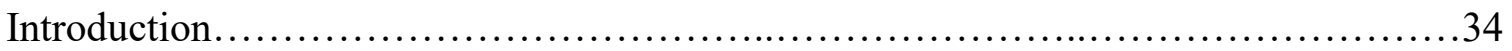

Methods...........................................................................

Results...............................................................42

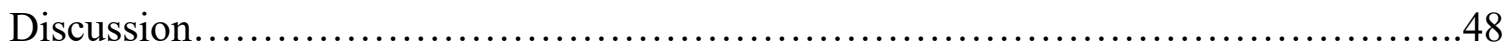

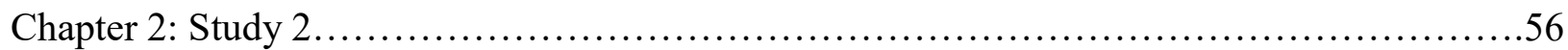

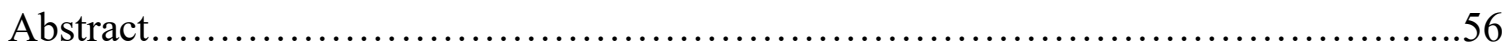

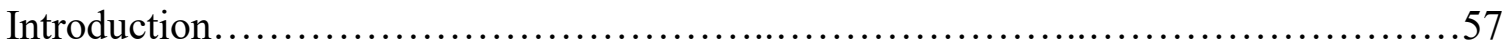

Methods.......................................................................60 


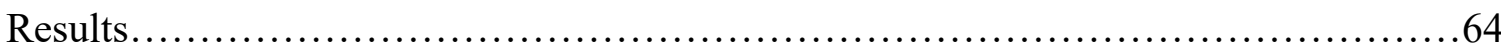

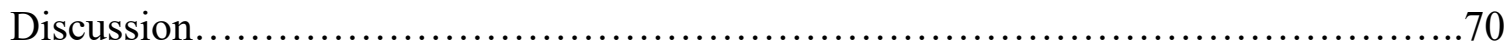

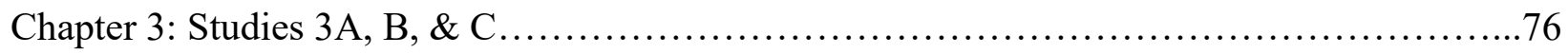

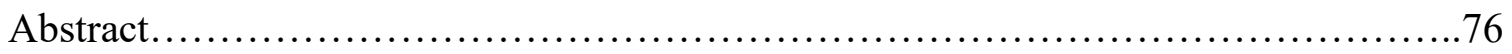

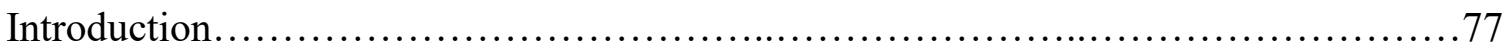

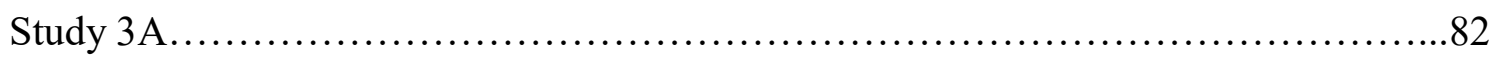

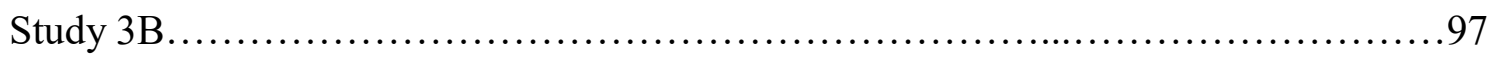

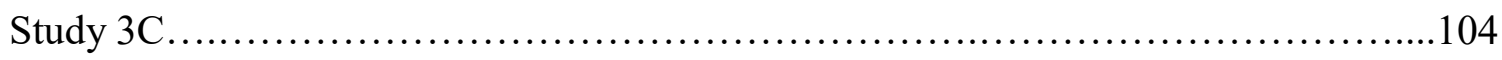

General Discussion............................................................111

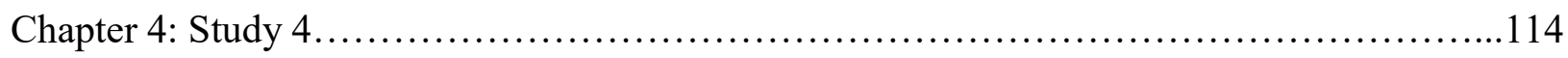

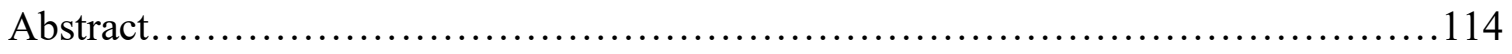

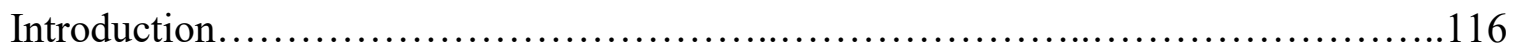

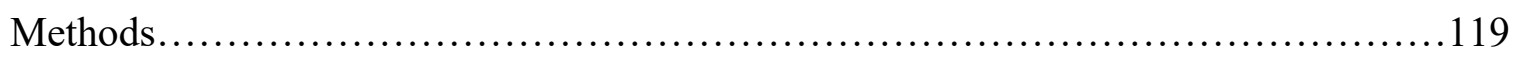

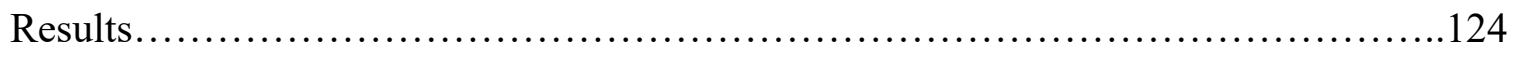

Discussion........................................................................ 134

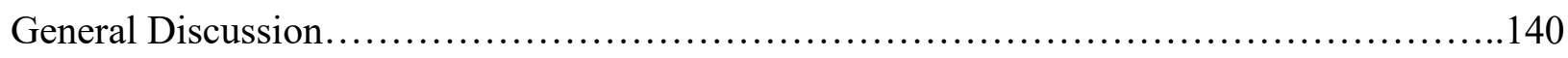

Limitations and Conclusions................................................. 146

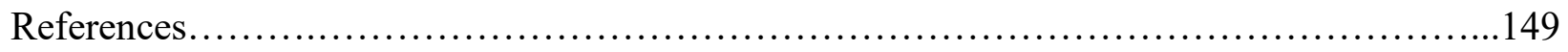

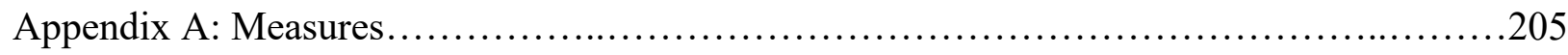




\section{List of Tables}

\section{Chapter 3 - Study 1}

Table 1. Promax Rotated Pattern Matrix for the CFQ..................................88

Table 2. Zero-order correlations comparing the relation between the CFQ and CFI and validation measures of cognitive flexibility/rigidity.............................. 91

Table 3. Partial correlations between the CFQ and validation measures of cognitive flexibility/rigidity after controlling for the Cognitive Flexibility Inventory.............91

Table 4. Partial correlations between the CFQ and depressive symptoms after controlling for other measures assessing aspects of cognitive flexibility/rigidity......................94 


\section{List of Figures}

\section{Chapter 1 - Study 1}

Figure 1. Differences $( \pm \mathrm{SEM})$ in (a) perseverative versus non-perseverative errors, (b) trials to first category, and (c) failures to maintain an attentional set among individuals in the stressor and control conditions.................................................. 43

Figure 2. Salivary cortisol levels $( \pm$ SEM) over time between individuals in the stressor and control conditions

\section{Chapter 2 - Study 2}

Figure 1. Mean ( \pm S.E.M.) gender differences in the frequency of different forms of traumatic events.

Figure 2. The moderating role of the BDNF Val66Met polymorphism and gender in the relationship between frequency of general traumas and frequency of perseverative errors on the WCST.

Figure 3. The moderating role of the BDNF Val66Met polymorphism and gender in the relationship between frequency of physical punishment and frequency of perseverative errors on the

WCST..... .68

Figure 4. The moderating role of the BDNF Val66Met polymorphism and gender in the relationship between frequency of emotional abuse and frequency of perseverative errors on the WCST. 


\section{Chapter 3 - Study 3B}

Figure 1. Moderating role of cognitive flexibility in the relation between dysfunctional attitudes and symptoms of depression (A), and negative cognitive style and depressive symptoms (B) 101

\section{Chapter 3 - Study 3C}

Figure 1. Multiple mediation analysis examining the mediating effect of threat and uncontrollability appraisals in the relationship between cognitive flexibility and depressive symptoms................................................. 108

Figure 2. Multiple mediation analysis examining the mediating effect of problem- and emotionfocused coping in the relationship between cognitive flexibility and depressive symptoms.

\section{Chapter 4 - Study 4}

Figure 1. Mean differences ( \pm S.E.M.) between the stressor and control groups in the intensity of negative (A) and positive (B) affect over time.

Figure 2. Mean differences ( \pm S.E.M.) between the stressor and control groups in perceived threat (A) and uncontrollability (B) over time. 126

Figure 3. Immediately post-stressor, the mediating role of threat appraisal in the relation between stressor condition and negative affect was moderated by differences in cognitive control.

Figure 4. Immediately post-stressor, the mediating role of uncontrollability in the relation between stressor condition and negative affect was moderated by differences cognitive resources............................................................... 128 
Figure 5. At 40 minutes post-stressor, the mediating role of threat appraisal in the relation between stressor condition and negative affect was moderated by differences cognitive control.

Figure 6. At 40 minutes post-stressor, the mediating role of uncontrollability in the relation between stressor condition and negative affect was moderated by differences cognitive control.

Figure 7. At 40 minutes post-stressor, the mediating role of uncontrollability in the relation between stressor condition and negative affect was moderated by differences cognitive

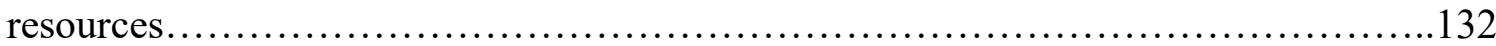

Figure 8. The mediating role of uncontrollability in the relation between stressor condition and plasma cortisol AUCi was moderated by differences in cognitive resources...........134 


\section{List of Appendices}

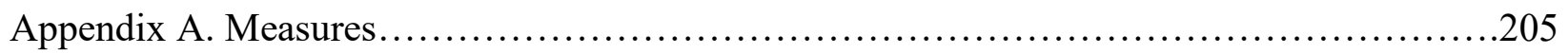




\section{General Introduction}

Depressive disorders are characterized by persistent negative mood, and are typically accompanied by repetitive negative thinking as well as negative biases in information processing, including attention, memory, and appraisal/interpretation processes (Gotlib \& Joorman, 2010; Joorman \& D’Avanzata, 2010; Matthews \& Macleod, 2005). Moreover, depressed individuals engage in ineffective coping and emotion regulation strategies, such as rumination, at the expense of proactive methods, such as problem-solving and cognitive reappraisal/restructuring (Joorman \& Gotlib, 2010; Matheson \& Anisman, 2003; NolenHoeksema, 2000). It is less clear why depressed individuals, or those at risk for the illness, engage in these self-defeating cognitive and behavioral responses.

To address this question, increased attention has been directed towards understanding the contribution of fundamental cognitive (executive) control processes in the regulation of emotions (Okon-Singer et al., 2015; Ochsner, Silvers, \& Buhle, 2012; Ochsner \& Gross, 2005; 2008), and how disturbances in these processes might favor the maintenance and exacerbation of negative affective states (Joormann, 2010; Joormann \& Gotlib, 2010). To be sure, reduced or impaired cognitive functioning has frequently been observed among depressed individuals, with the most consistently reported deficit being that of cognitive flexibility (Austin et al., 1992; Beats et al., 1996; Elliot et al., 1997; Grant et al., 2001; Kinderman et al., 2000; Martin et al., 1991; Merriam et al., 1999; Purcell et al., 1997; Trivedi \& Greer, 2014). Importantly, the disturbed cognitive flexibility (and cognitive functioning, in general) is not only present during the course of a depressive episode, but persists following illness remission (Trivedi \& Greer, 2014), and was associated with increased risk for relapse (Alexopoulos et al., 2000; Majer et al., 2004) 
Although a general deficit in cognitive flexibility is a central characteristic of depressive pathology, this disturbance appears to be especially pronounced in the context of negative (as opposed to positive or neutral) emotional information (Deveney \& Deldin, 2006; Williams, Mathews, \& MacLeod, 1996; Joormann, 2004). Thus, it was postulated that the specific inability to disengage from negative emotional information might be a defining feature of depression, distinguishing this illness from other forms of psychopathology (Joormann, Yoon, \& Zetsche, 2007; Joormann, 2010; Koster et al., 2010). Moreover, impaired cognitive control has been associated with more frequent utilization of rumination and reduced engagement in cognitive reappraisal, characteristic of depressive pathology (Joorman \& Gotlib, 2010; Joorman, 2010)

Stressful events can have a considerable impact upon cognitive functioning (Arsten, 2009, Arsten et al., 2015; Lupien, 2007; Lupien, McEwen, \& Gunnar, 2009; Sandi, 2013) and play a fundamental role in the evolution of depressive disorders (Kessler, 1993). Relatively few studies, however, have examined the effects of acute and chronic stressors on cognitive flexibility, especially in humans, and the available data from animal based studies have provided inconsistent findings, partly owing to the utilization of different stressor protocols and behavioral tasks employed to assess cognitive flexibility (Hurtubise \& Howland, 2017).

The present research was aimed at an analysis of the relationship between different stressors (both acute and chronic) and cognitive flexibility, as assessed by a commonly used behavioral task (i.e., the Wisconsin Card Sorting Task; WCST), and the processes mediating these relationships. It was also of interest to determine whether individual differences, including gender and genetic background, moderated the relationship between stressful events and cognitive flexibility. As alluded to earlier, disturbances in cognitive control and flexibility may underlie the difficulties of emotional regulation that are characteristic of depressive disorders. 
Thus, yet another aim of the present research was to determine whether diverse stressor-elicited emotional responses would be differentially expressed with respect to cognitive inflexibility, and to what extent this would be related to elevated symptoms of depression. In doing so, a selfreport measure of cognitive flexibility (the CFQ) was developed that captured the fundamental cognitive processes reflected in behavioral tasks assessing cognitive flexibility (i.e., the ability to modify cognitive content and behavioral strategies), and applied these processes to stressful events and depressive symptoms, and whether the CFQ predicted emotional and neuroendocrine (i.e., cortisol) responses following an acute psychosocial stressor.

\section{Defining and assessing cognitive flexibility}

Cognitive flexibility is generally defined as the ability to adjust or modify cognitive content ("mental sets") and behavioral strategies in response to changes in environmental demands (Cragg \& Chevalier, 2012; Ionesca, 2012). Given such a broad definition, it may not be surprising that this ability can be expressed in many different ways. For example, in the case of decision-making, a display of cognitive flexibility might comprise weighing the costs and benefits of several different actions. Similarly, generating and comparing several different solutions to a problem, or even engaging in multi-tasking, might reflect flexible cognitive processing. As will be discussed later, cognitive flexibility might also be expressed as the ability to modify appraisals and coping strategies when faced with stressful circumstances, and an inability to do so may be a central characteristic of psychopathology, including depressive illness. In this regard, however, the context or the specific demands of a particular situation may be an important factor with respect to how cognitive flexibility is ultimately expressed and assessed. 
At the most fundamental level, this ability is considered to reflect multiple cognitive (executive) control processes, including attention, working memory, conflict and error monitoring as well as response selection and inhibition (Dajani \& Uddin, 2015). Disruptions in any of these cognitive control processes might thus result in a loss of cognitive flexibility, which is generally expressed as perseverative thoughts and behaviors. Cognitive flexibility has predominantly been assessed through various behavioral tasks, including set-shifting, taskswitching, and reversal-learning paradigms (also referred to as affective shifting). Importantly, although each of these tasks was designed to measure the construct of cognitive flexibility, they differ considerably in their characteristics and demands. As such, performance on each task has been associated with distinct neural activation patterns and was affected by variations in different neurotransmitter systems (Arsten, 2011; Klanker et al., 2013; Kehagia, Murray, \& Robbins, 2010). The present research was primarily concerned with set-shifting, as this has been the most consistently reported type of deficit among depressed individuals. Accordingly, this review primarily focuses on the neurobiological aspects of this form of cognitive flexibility.

\section{Wisconsin card sorting task (WCST)}

The WCST is the most commonly used task to assess set-shifting. On the WCST, the individual is asked to match test cards to reference cards based on color, shape, or number of stimuli on the cards. Feedback is provided after each match, enabling the individual to acquire the correct rule of classification, or attentional (cognitive) set. After a fixed number of correct matches, the rule is changed, without notice, and the individual must shift their attention to a new method of classification. The main outcome measure of the WCST is the frequency of perseverative errors, which refers to number of trials in which the participant continues to respond to a previously correct, but now incorrect, stimulus. Non-perseverative errors, by 
contrast, refers to all other types of errors, including those associated with an inability to acquire or maintain an attentional (cognitive) set.

\section{Intra- and extra-dimension (ID/ED) shift task}

An analogue to the WCST is ID/ED shift task, which uses only two stimulus dimensions (i.e., color and shape) (Downes et al., 1989). The ID/ED task also decomposes the specific processes inherent to the WCST. Specifically, in the ID/ED task, an intra-dimensional (ID) shift involves shifting between new stimuli but within the same perceptual dimension, and reflects the acquisition of an attentional set. An extra-dimensional (ED) shift, involves a shift in responding from one dimension (e.g. color) to another discrimination rule (e.g. shape), and this is referred to as a shift in an attentional set. Similar to the WCST, set-shifting difficulties are measured by the frequency of perseverative responding to a previously, but no longer, relevant stimulus.

\section{Set-shifting in animals}

The most commonly used task to assess set-shifting in rodents is the Attentional SetShifting Task (ASST) (Birrell and Brown, 2000; Bissonette et al., 2008). In the ASST, rodents are initially trained to dig for a food reward in one of two bowls based on either an olfactory (e.g., cinnamon and cumin) or medium/texture (e.g., wood shavings and saw dust) cue. During ID shift phase, rodents are presented with new exemplars but within the same relevant dimension. In the ED shift phase, the relevant dimension (e.g., olfactory) becomes irrelevant, and a new dimension becomes relevant (texture). Attentional set-shifting deficits are assessed by the amount of trials in which the rat (or mouse) continues to respond to a previously rewarded stimulus. 


\section{Neural circuitry of cognitive flexibility}

Cognitive flexibility is a complex ability resulting from the coordinated effort of multiple cognitive control processes. From this perspective, it may not be surprising that this ability is governed by multiple brain regions. To be sure, neuroimaging studies have demonstrated widespread activation of fronto-parietal and fronto-striatal neural circuits during performance of various behavioral tasks assessing set-shifting (Braver et al., 2003; Dove et al, 2000; Hamsphire \& Owen, 2006; Hyafil et al., 2009; Monchi et al., 2001; Smith et al., 2004). Likewise, lesions of various prefrontal, parietal, and striatal regions (or pathways connecting these regions) can result in impaired set-shifting performance (Birrell \& Brown, 2000; Bissonette et al., 2008; Block et al., 2007; Dias et al. 1996a, b; 1997; Fox et al., 2003; Milner, 1963; Ng et al., 2007; Newman \& McGaughy, 2011; Oualian \& Gisquet-Verrier, 2010; Owen et al., 1991; Ragozzino et al., 2002; Robbins, 2007). In the present section, a review is presented of the main brain regions involved in set-shifting and the unique cognitive process that each brain region might contribute to this ability

Prefrontal Cortex (PFC)

Neuroimaging studies have consistently demonstrated activation of prefrontal cortical regions during performance on the WCST and the ID/ED shift task (Braver et al., 2003; Dove et al, 2000; Hamsphire \& Owen, 2006; Hyafil et al., 2009; Monchi et al., 2001; Smith et al., 2004). Furthermore, lesions to the various regions of the PFC have been accompanied by perseverative responding, characterized by the inability to disengage from a previously, but no longer, relevant stimulus (Dias et al. 1996a, b; 1997; Milner, 1963; Owen et al., 1991).

The PFC can be functionally categorized into distinct cortical regions, with each contributing to a specific cognitive process involved in set-shifting. For example, performance 
on the WCST has been associated with sustained activation of the dorsolateral PFC (Monchi et al., 2001), which is consistent with this region's role in working memory processes, including the maintenance and updating of currently relevant information (Hamsphire \& Owen, 2006; Monchi et al., 2001). In contrast, transient activation of the ventrolateral PFC (or the inferior frontal sulcus) occurred specifically during the receipt of negative feedback on the WCST, which corresponds to the need to adopt a shift in attention or a new behavioral strategy (Konishi et al., 1998; 1999a, b; Monchi et al., 2001; Nakahara et al., 2002). Findings from such studies pointed to this region being involved in selecting currently relevant cognitive-behavioral strategies, while inhibiting those that are no longer relevant or appropriate (Aron et al., 2004; Monchi et al., 2001; Petrides, 2005).

\section{Posterior Parietal Cortex}

The posterior parietal cortex (PPC) appears to play a supporting role to the lateral PFC in set-shifting (Braver et al., 2003; Fox et al., 2003; Monchi et al., 2001; Hampshire \& Owen, 2006), with lesions to this region producing a preservative-type deficit, similar to that evident following medial PFC lesions in rodents (Fox et al., 2003). Activation of the PPC and the posterior PFC has been observed at the point where a newly appropriate behavioral strategy is being acquired during the course of a set shift (Monchi et al., 2001), suggesting that these regions might be important for stimulus response mapping, or the ability to acquire newly appropriate (or relevant) cognitive strategies (Fox et al., 2003; Monchi et al., 2001).

Beyond the PFC and PPC, transient activation of the anterior cingulate cortex (ACC) has been observed during the receipt of negative feedback in the WCST indicating that the current behavioral strategy is no longer appropriate (Monchi et al., 2001). The ACC, however, may not be directly involved in initiating an attentional shift, but rather might play a greater role in 
conflict monitoring and error processing (Botvinick et al., 2004; Kerns et al., 2003). In line with this view, lesions to the ACC in nonhuman primates and rodents produced a selective deficit in ID shifting and not on ED shifting (thus, not a set-shifting impairment per se), and were associated with more trials and errors required to complete a set-shifting task ( $\mathrm{Ng}$ et al., 2007; Newman \& McGaughy, 2011; Oualian \& Gisquet-Verrier, 2010). Thus, the ACC may be important for signaling that an error had been made, and this information is likely transmitted to dorso- and ventro-lateral prefrontal regions, which then adjust cognitive and behavioural strategies accordingly (Kerns et al., 2003; McDonald et al, 2000).

Cognitive flexibility is not limited to fronto-parietal regions, and seems to be dependent on optimal striatal functioning (Robbins, 2007). Inactivation of the dorsomedial striatum or the nucleus accumbens core (NAc) as well as asymmetrical disconnections between the PFC and NAc or PFC and mediodorsal nuclei of thalamus (MD), was associated with impaired setshifting performance in rats (Block et al., 2007; Ragozzino et al., 2002). In line with these findings, set-shifting difficulties were reported among humans with focal lesions to the basal ganglia as well as among individuals during the early phase of Parkinson's disease, which is characterised by dorsal striatum dysfunction (Cools et al., 1984, 2001a,b, 2003; Downes et al., 1989; Gauntlett-Gilbert et al., 1999; Owen et al., 1993). Importantly, the striatum appears to contribute to a different aspect of cognitive flexibility relative to the PFC or PPC. Specifically, unlike PFC or PPC damage, which is associated with perseveration to a previously relevant attentional set, striatal damage seems to be associated with the inability to acquire and maintain a newly relevant attentional set (Ragozzino et al., 2002). Thus, while the PFC regions might be primarily responsible for inhibiting inappropriate cognitive strategies, the striatum may be responsible for determining and maintaining a novel strategy. 


\section{Neurochemical regulation of cognitive flexibility}

Pharmacological studies indicated that monoamines, including dopamine, norepinephrine, and serotonin, played a central role in the regulation of cognitive flexibility, including set-shifting (Arsten, 2011; Klanker et al., 2013; Kehagia, Murray, \& Robbins, 2010). It appeared that each neurotransmitter system regulated unique cognitive (or biological) processes involved in this ability, and could have dissociable roles in mediating aspects of cognitive flexibility (Roberts, \& Robbins, 2007; Robbins \& Roberts, 2007), although it is understood that these actions will also vary across brain regions. Furthermore, recent evidence demonstrated that optimal levels of cognitive flexibility might not be restricted to monoamine variations, and likely involves other neurobiological mechanisms, including brain-derived neutrophic factor (BDNF) (D’Amore et al., 2013; Ortega et al., 2013; Sakata et al., 2013). The present section will briefly synthesize the literature concerning monoamine and BDNF manipulations on cognitive flexibility, with a specific focus on the unique processes these neurochemicals might contribute to this ability.

\section{Dopamine}

Dopamine (DA) is known to play a role in cognition, especially in reward-based learning (e.g., reward prediction error) and working memory (Cools \& D’Esposito, 2011; Montague et al., 2004; Schultz et al., 1997), and thus might be involved in cognitive flexibility. Consistent with this view, in animals, global and brain region-specific (e.g., PFC) depletion of DA impaired performance on behavioural tasks assessing cognitive flexibility (Klanker et al., 2013). Likewise, among un-medicated individuals with early Parkinson's Disease (PD), which is characterized by significant dopaminergic cell loss in the dorsal striatum (Damier et al., 1999; Kish et al., 1988), set-shifting impairments were apparent (Cools \& Robbins, 2004). Moreover, drugs that increase 
fronto-striatal DA levels could improve set-shifting performance, especially among individuals who exhibited a deficit in this ability (Cools \& Robbins, 2004; Mehta et al., 2004)

The involvement of DA in cognitive flexibility appears to be mediated by $\mathrm{D}_{1}$ and $\mathrm{D}_{2}$ receptor signalling, as blockade of either of these receptors in the medial PFC of rats produced preservative responding on a strategy-shifting task (Ragozzino, 2002), whereas D1 stimulation facilitated this ability (Nikiforuk, 2012). Furthermore, whereas blockade of D2 receptors in humans impaired set-shifting, stimulation of these receptors enhanced this ability (Kimberg et al., 1997; Mehta et al., 2001; 2004; van Holstein et al., 2011). Considering such findings, it was suggested that D1 and D2 receptors might have complimentary actions in relation to cognitive flexibility (Floresco \& Magyar, 2006). Specifically, phasic DA neuronal firing, which is associated with high DA levels, stimulates D2 receptors, and renders the PFC more flexible, allowing it to process multiple stimuli and mental representations (Seamans \& Yang, 2004). By contrast, tonic DA neuronal firing, which is associated with moderate extra-cellular concentrations of DA, activates D1 receptors and facilitates the ability to focus on individual stimuli (or mental representation), while preventing accessibility of potential distractions (Seamans \& Yang, 2004). In the context of cognitive flexibility, phasic firing stimulating D2 receptors might then facilitate the ability of the PFC to disengage from previous cognitive strategies and compare the viability of alternative cognitive or response options, whereas tonic firing, that stimulates D1 receptors, might promote the stability of a new cognitive strategy (Floresco \& Magyar, 2006).

\section{Norepinephrine}

Norepinephrine (NE) manipulations using medications, such as clonidine, guanfacine and beta-blockers (e.g., propranolol), have generally had no detectable effects on cognitive flexibility 
in humans (Chaimberlain \& Robbins, 2013). The lack of any action was attributed to small sample sizes or that cognitive flexibility tasks used for humans might be too simple and thus not suitable to differentiate differences based on pharmacological manipulations (Chaimberlain \& Robbins, 2013). However, animal studies have implicated NE involvement in this ability. Lesions of the dorsal noradrenergic ascending bundle (Tait et al., 2007) and deafferentation of NE terminals in the medial PFC (McGaughy et al., 2008) was associated with impaired setshifting in rats. Moreover, chronic treatment with a NE reuptake blocker (desipramine) in rats enhanced set-shifting (Lapiz et al., 2007), although excessively high NE release can have the opposite effect (Cain et al., 2011; Newman et al., 2008).

Although it is not entire clear as to what the role of NE is in cognitive flexibility, it is possible that this neurotransmitter acts through its involvement in attention and arousal (Arnsten, 2000; Aston-Jones \& Cohen, 2005; Kehagia et al., 2010). In essence, NE appears to have an "inverted U" - shaped relationship with cognition, with both low and high NE release impairing cognitive functioning, depending on the engagement of different types of receptors. Norepinephrine has its highest affinity for $\alpha 2$-adrenergic receptors, and lower affinity for $\alpha 1$ - and $\beta$-adrenergic receptors (Arnsten, 2000). Moreover, the levels of NE that are released during alert, non-stressed periods optimize prefrontal cognitive functioning by engaging $\alpha 2 \mathrm{~A}$-receptors (Arnsten \& Goldman-Rakic, 1985), whereas the high levels of NE that are released in response to a stressor impair PFC-related cognitive processes by stimulating lower-affinity $\alpha 1$-receptors and $\beta 1$-receptors (Birnbaum et al., 1999; Ramos et al., 2005). In line with these findings, blockade of $\alpha 1$ receptors in rats improved set-shifting, whereas chronic $\alpha 2$ receptor antagonism had the opposite effect (Rowe et al., 1996). Likewise, it has been reported that intra-medial PFC infusions of a postsynaptic $\alpha 1$ receptor antagonist prevented the attentional set-shifting 
improvement observed following systemic injections of an adrenergic auto-receptor antagonist (atipamezole) in rats (Lapiz and Morilak, 2006).

\section{Serotonin}

The involvement of serotonin (5-HT) in cognitive flexibility is less clear than that of DA and NE. In humans, acute tryptophan depletion (ATD), which significantly reduces central 5-HT, had no effects on set-shifting performance (Evers et al., 2005a, b; Gallagher et al., 2003; Hughes et al., 2002; 2003; Murphy et al., 2002; Talbot et al., 2006, but see Rogers et al., 1999). In animals, however, acute administration of selective 5-HT reuptake inhibitors, such as escitalopram and fluoxetine, as well as systemic treatment with a 5- $\mathrm{HT}_{2 \mathrm{~A}}$ antagonist enhanced set-shifting (Baker et al., 2011; Nikiforuk \& Popik, 2011). Furthermore, acute systemic administration of a 5-HT6 receptor agonist in rats facilitated ED set shifting (Burnham et al., 2010), and attenuated the setshifting deficit associated with 5-HT depletion elicited by phencyclidine (Rodefer et al., 2008), whereas administration of a $5-\mathrm{HT}_{7}$ receptor antagonist improved set-shifting performance (Nikiforuk, 2012). Thus, the effects of 5-HT neurotransmission on set-shifting ability might be mediated by 5- $\mathrm{HT}_{2} \mathrm{~A}, 5-\mathrm{HT}_{6}$, and 5- $\mathrm{HT}_{7}$ receptors (Burnham et al., 2010; Baker et al., 2011; Nikiforuk, 2012).

\section{Brain-derived neurotrophic factor}

Cognitive flexibility is dependent on the structural and functional integrity of prefrontal cortical neurons. Thus, processes that might facilitate neuronal functioning within this brain region might be involved in optimizing cognitive flexibility. In this regard, BDNF is densely expressed in the hippocampus as well as the PFC (Pezawas et al. 2004), and influences neuronal survival, dendritic and axonal growth, and synaptic plasticity (Pang \& Lu 2004; Poo, 2001). This 
neurotrophic factor has frequently been implicated in various hippocampal-mediated functions, such as learning and memory (Cunha, 2010). However, its potential involvement in cognitive flexibility has hardly been explored. Nevertheless, intra-striatal injections of BNDF in mice facilitated the acquisition of strategy shifting, reflected by diminished response perseveration in the previously acquired strategy (D'Amore et al., 2013). Along similar lines, attenuation of activity-dependent BDNF expression impaired spatial memory reversal and contextual memory extinction in rats (Sakata et al., 2013), and reduced BDNF levels were accompanied by impaired reversal learning following high doses of nicotine in mice (Ortega et al., 2013). Clearly, additional research is needed concerning the contribution of BDNF to cognitive flexibility, and whether targeting this neurotrophic factor may play an important role in treating psychiatric conditions that are characterized by a deficit in this ability, including depressive disorders. Nonetheless, the data currently available appear to be promising.

\section{Genetics of Cognitive flexibility}

Gene-association studies have identified several gene variants, or single nucleotide polymorphism (SNPs), that might contribute to individual differences in cognitive flexibility, with the most current evidence focusing on SNPs related to DA regulation and functioning. The Val158Met COMT gene polymorphism has been associated with the efficiency of DA degradation in the PFC, with the Met allele being related to less COMT activity than the $\mathrm{Val}$ allele, and thus presumably higher PFC DA levels (Chen et al., 2004). Individuals homozygous for the Met allele performed better on the WCST, reflected by fewer perseverative errors, than those carrying the Val allele (Bruder et al., 2005; Egan et al., 2001; Joober et al., 2002; Malhotra et al., 2002; Mattay et al., 2003; Rosa et al., 2004), although several studies failed to find this association (Ho et al., 
2005; Taerk et al., 2004; Tsai et al., 2003). Another DA-related gene polymorphism that has been associated with cognitive flexibility, the $C 957 T$ polymorphism of the DRD2 gene, affects $\mathrm{D}_{2}$ receptor availability. Individuals carrying the $T$ allele have higher striatal $\mathrm{D}_{2}$ receptor availability compared to those carrying a $C$ allele (Hirvonen et al., 2009), and carriers of the $C C$ genotype, compared with carriers of the CT/TT genotypes, displayed reduced set-shifting ability in the WCST (Rodriguez-Jimenez et al., 2006).

Beyond gene polymorphisms related to DA, relatively few studies have examined the contribution of other polymorphisms to individual differences in cognitive flexibility, although some evidence suggested that the serotonin transporter linked polymorphic region (5-HTTLPR) might be involved. The 5-HTTLPR regulates the efficacy of the 5-HT transporter and, in humans, the short $(s)$ allele is associated with reduced expression of 5-HT transporters compared to the long ( $l$ ) allele (Canli \& Lesch, 2007), and carriers of the $s$ allele s might be better at set-shifting (Borg et al. 2009).

There has been evidence that mutations of the BDNF gene might also predict differences in cognitive flexibility. In this regard, the Met allele of the BDNF Val66Met polymorphism has been associated with reduced BDNF expression, hippocampal and dorsolateral PFC volume reductions, poor performance on hippocampal-dependent memory tasks, increased stressreactivity, and increased risk for mood and anxiety disorders (Bueller et al., 2006; Chen et al., 2006; Egan et al., 2003; Hariri et al., 2003; Pezawas et al., 2004). Based on these findings, it might be expected that Met carriers would also exhibit reduced cognitive flexibility compared to $\mathrm{Val}$ homozygotes. Consistent with this view, carriers of the Met allele performed significantly worse than $\mathrm{Val} / \mathrm{Val}$ homozygotes on the WCST, but this was only true among individuals with bipolar disorder and not controls or those with schizophrenia (Rybakowski et al., 2003). A more recent 
study, however, failed to find any association, even among individuals with bipolar disorder (Zeni et al., 2013). Limited data supporting a link between BDNF mutations and cognitive flexibility in healthy humans are currently available.

The impact of certain genetic variants on cognitive and emotional functioning may depend on environmental factors (Belsky \& Pluess, 2009).Several studies indicated that the impact of early life experiences, including stressful and traumatic events, on later-life cognitive and affective functioning was moderated by the 5-HTTLPR as well as the BDNF Val66Met polymorphism (Caldwell et al., 2013; Caspi et al., 2003; Clasen et al., 2012; Conway et al., 2013; Gatt et al., 2009; Hosang et al., 2014). Surprisingly, however, studies have not examined the potential moderating effects of these or other polymorphism in relation to proximal and early life stressors and cognitive flexibility.

\section{Stressor effects on cognitive flexibility}

Stressful events can have multiple effects on cognitive functioning, with the outcome (i.e., facilitating or impairing) depending on complex interactions between the characteristics of the stressor (e.g., intensity, duration, and controllability) and the specific aspect of cognition under study (Arsten, 2009; Arsten et al., 2015; Lupien, 2007; Lupien, McEwen, \& Gunnar, 2009; Sandi, 2013). Generally, mild to moderate stressors facilitate cognitive functioning, including the acquisition and maintenance of (especially threat-related) information (but impair its retrieval) or when the cognitive or mental operations are relatively simple (i.e., low cognitive load) (Cahill, Gorski, \& Le, 2003; Hupbach \& Fieman, 2012; Kuhlmann, Piel, \& Wolf, 2005; Roozendraal, McEwen, Chattarji, 2009; Schoofs, Wolf, \& Smeets, 2009; Stauble, Thompson, \& Morgan, 2013). More severe, or chronic, stressors, by contrast, tend to impair various aspects of 
cognition, including executive functions (e.g., working memory), although these events can improve performance on well-rehearsed tasks (Hurtubsie \& Howland, 2016; Marin et al., 2011; McEwen \& Morrison, 2013). In contrast to these findings, the data relevant to the effects of stressors on cognitive flexibility are relatively limited, especially in humans. Furthermore, it is difficult to draw conclusions from the available data, since each study used different stressors and different tasks to assess cognitive flexibility. Still, it is important to recognize the effects of these stressor experiences on cognitive flexibility.

\section{Acute stressors}

Under normal, non-stress conditions, the PFC is thought to exert "top-down" control over various sensory and limbic structures, allowing for flexible cognitive behavioral responses (Miller \& Cohen, 2001). When stressors are encountered, prefrontal cognitive functions may be compromised as a result of excessive DA and NE release, and "bottom-up" processing, mediated by structures such as the amygdala and basal ganglia, predominates (Arnsten, 2009). The consequence of this switch from top-down to bottom-up processing results in fast, well-learned, and reflexive behavioural responses, at the expense of more deliberate and flexible behaviors. From this perspective, it would be expected that cognitive flexibility would be impaired under stressful conditions. Surprisingly, however, only one study examined the effects of an acute stressor on cognitive flexibility, indicating that a group version of the TSST was followed by reduced set-shifting ability on the WCST, but only among males (Shields et al., 2016). The available animal data are also limited, but indicated that strong acute stressors (e.g., acute tail pinch) impaired set-shifting (Butts et al, 2013), whereas less intense stressors (e.g., 30 minutes of restraint stress) had no effect on this ability (Thai et al., 2013). Clearly, more research is needed 
to determine under which conditions acute stressors can facilitate or impair cognitive flexibility, and the processes mediating these effects, although provisionally it might be that performance is linked to stressor severity.

\section{Chronic stressors}

In humans, only one study has examined the relation between chronic stressors and cognitive flexibility. One month of self-reported stress impaired set-shifting, and this deficit was associated with disrupted functional connectivity between several regions within the PFC and posterior parietal areas (Liston et al., 2009). Interestingly, in this study, reductions in perceived distress over the subsequent month was accompanied by improved set-shifting ability and elevated fronto-parietal connectivity. Given the correlational nature of the study, it is not possible to determine whether the chronic stressor resulted in impaired set-shifting or whether reduced cognitive flexibility lead to greater perceived stress.

Animal studies have provided evidence for a more direct role of chronic stressors on cognitive flexibility. Various chronic stressor protocols, including chronic unpredictable stressors (CUS), repeated restraint stressors, and maternal separation, reliably produced setshifting deficits in rodents (Bondi et al., 2008; 2010; Liston et al., 2006; Nikiforuk \& Popik, 2011), although chronic intermittent cold stressors had no effect on this ability (Lapiz-Bluhm et al., 2009). Moreover, the impairments in set-shifting observed following chronic stressor exposure might be mediated by altered monoaminergic functioning. Specifically, chronic pretreatment with either a selective NE reuptake blocker (desipramine) or selective 5-HT reuptake blockers (e.g., citalopram and escitalopram) prevented the set-shifting deficits induced by CUS as well as repeated restraint stress (Bondi et al. 2008, 2010; Nikiforuk \& Popik, 2011).

Additionally, administration of a D1 agonist reversed set-shifting impairments, suggesting that 
altered DA functioning might also contribute to stress-induced impairments in set-shifting (Nikiforuk, 2012).

Additionally, chronic stressors produce marked reductions of dendritic dendrite length, branching, and spine density in the medial PFC of rodents (Holmes \& Wellman, 2009), which may have been due to excessive and prolonged exposure to glucocorticoids (Wellman, 2001). It is possible that in addition dysregulation of central monoaminergic activity, impaired cognitive flexibility following chronic stressors might be mediated by excessive release of glucocorticoids, resulting in morphological changes within the prefrontal cortical regions (Listen et al., 2006). Although the data are limited in this regard, inhibiting corticosterone synthesis prior to daily repeated stressors prevented set-shifting impairments that normally followed repeated restraint stress (Nikiforuk and Popik, 2011). As well, a chronic restraint stressor induced a selective impairment in set-shifting in rodents and a corresponding retraction of apical dendritic arbors in the medial PFC (Liston et al., 2006)

Early life stressors

Cognitive flexibility skills typically begin developing in early childhood, with a sharp increase in the competence in this ability appearing between 7 and 9 years of age (Dajani \& Uddin, 2015). By 10 years of age, cognitive flexibility is largely developed (Dick, 2014), although improvements in this ability can persist throughout adolescence and into adulthood (Anderson, 2002; Hunter \& Sparrow, 2012), reaching a peak between the ages of 21 and 30 years (Cepeda et al., 2001). The development of cognitive flexibility is thought to reflect the development and maturation of the PFC (Dajani \& Uddin, 2015), and thus, factors which alter normal PFC development might compromise cognitive flexibility. Surprisingly, relatively few studies have examined the relationship between early life stressors and cognitive flexibility 
across different developmental stages. Nevertheless, the current evidence suggests that the relationship between early life events and later cognitive flexibility differences might depend on the nature, particularly the severity, of the events experienced. Specifically, unpredictable environments (e.g., frequent changes in residence or parent's job/work status) or low socioeconomic status have been aligned with enhanced set-shifting performance (Chen \& Miller, 2012; Mittal et al., 2015). Childhood trauma (e.g., physical abuse and neglect), by contrast, has frequently been associated with difficulties in attentional set-shifting in humans (George et al., 2015; Mothes et al., 2015; Spann et al., 2012).

As described earlier, the relationship between early life stressors and later-life cognitive and affective functioning was moderated by the 5HTTLPR and the Val66Met polymorphism of the BDNF (Caldwell et al., 2013; Caspi et al., 2003; Clasen et al., 2012; Conway et al., 2013; Gatt et al., 2009; Hosang et al., 2014). Given the involvement of 5-HT and BDNF in the cognitive flexibility, it is possible that 5HTTLPR and the BDNF polymorphism, as well as gene polymorphisms related to DA and NE functioning, might moderate the relationship between early life stressors and cognitive flexibility as well. This possibility, however, has yet to be investigated.

\section{Cognitive (in)flexibility in depressive disorders}

Depressive disorders have frequently been associated with disturbances in cognitive flexibility, with the most consistent deficits being observed on set-shifting tasks (Austin et al., 1992; Beats et al., 1996; Elliot et al., 1997; Grant et al., 2001; Kinderman et al., 2000; Martin et al., 1991; Merriam et al., 1999; Purcell et al., 1997).The disturbance in cognitive flexibility (or set-shifting) appears to be dependent on the individuals age, severity of depressive symptoms, 
and previous depressive episodes, with the most pronounced deficits being among severely depressed (e.g., melancholic) or elderly individuals (Austin et al., 1999; Grant et al., 2001;

Ottowitz et al., 2002). Importantly, diminished cognitive flexibility (and cognitive/executive control, in general) has not only been observed during the acute phase of depressive illness, but was also present during remission (Paelecke-Haberman et al., 2005; Paradiso et al., 1997), and has been suggested to increase the likelihood of depressive relapse (Alexopoulos et al., 2000). Surprisingly, there appears to be an absence of neuroimaging studies examining the neural correlates of cognitive flexibility (i.e., performance on tasks assessing this ability) among depressed individuals. Thus, it can only be speculated that in light ofthe involvement of frontostriatal and fronto-parietal circuits in set-shifting (Monchi et al., 2001), the impairments in cognitive flexibility present among depressed individuals might be accompanied by dysfunction of these neural circuits. Supporting this view, neuroimaging studies have reported reductions in grey matter volume in prefrontal regions (reviewed in Drevets et al., 2008) and striatal regions (Baumann et al., 1999; Husian et al., 1991; Krishnan et al., 1992; Pizzagalli et al., 2009b) among depressed individuals. As well, depressed individuals tend to exhibit reduced cerebral blood flow (CBF) and glucose metabolism in dorsal prefrontal regions, including the dorsolateral and dorsomedial PFC and the dorsal ACC (Baxter et al 1989; Drevets, 2001, 2001), and these abnormalities can be reversed with antidepressant treatment (Bench et al 1995; Mayberg et al 1999). This said, these data do not speak to whether these regions are linked to set-shifting disturbances that may or may not occur in depressed individuals.

Aberrant dorsal PFC and ACC activity has also been observed under cognitive challenge (i.e., during performance on cognitive tasks) among depressed individuals, although the direction of these changes (i.e., hypo- verse hyper-activation) varies across studies (Barch et al., 2003; 
Elliot et al., 1997; George et al., 1997; Harvey et al., 2005; Hugdahl et al., 2004; Okada et al., 2003; Videbech et al., 2003; 2004). Interestingly, when depressed individuals perform worse on cognitive tasks relative to non-depressed individuals, they displayed attenuated activity in the dorsolateral PFC and ACC (Elliott et al 1997; Okada et al 2003). However, when performing to the level of non-depressed individuals (but not better), those who were depressed exhibited greater activity in these regions (Harvey et al., 2005; Matsuo et al., 2007; Walter et al., 2007). Thus, it was suggested that depressed individuals might need to recruit more cognitive (neural) resources in order to match the performance of non-depressed individuals on effortful cognitive tasks (Harvey et al., 2005). Such cognitive efforts may place heavy demands on depressed individuals' cognitive/neural resources, leading to progressive exhaustion of these resources, ultimately leading to diminished cognitive functioning (Harvey et al., 2005). To extend this notion to cognitive flexibility, depressed individuals, and perhaps those vulnerable to depression, might need to utilize more cognitive (neural) resources in order to shift their attention away from inappropriate or negative emotional information and, if these resources become depleted, perseverative response styles might ensue.

The disturbance in cognitive flexibility appears to be especially pronounced in the present of negative emotional information (Deveney \& Deldin, 2006; Joormann, 2004; Murphy, Michael, \& Sahakian, 2012). For instance, in an emotional variant of the WCST, depressed individuals exhibited greater difficulties shifting their attention away from negative emotional stimuli (i.e., more perseverative errors) compared to non-depressed individual, whereas the opposite pattern was observed for positive information (Deveney \& Deldin, 2006). To be sure, this specific difficulty in disengaging from negative emotional information might serve to maintain negative cognitive and emotional states that define depressive disorders (Joormann et 
al., 2007; Joormann, 2010). Accordingly, the remainder of the present review discusses the various behaviors through which disturbances in cognitive flexibility might be manifested among depressed individuals, and the potential neural correlates underlying these behaviors.

\section{Attention and Memory}

Attentional biases have been well-documented in depressive disorders (Mathews \& MacLeod, 2005), characterized by a preferential bias toward negative emotion information (Broomfield et al., 2007; Erickson et al., 2005; Gotlib et al., 2004; Murphy et al., 1999; Segal et al., 1995; Williams et al., 1996). It appears that, among depressed individuals this bias occurs in later, rather than early stages of information processing (Foland-Ross \& Gotlib, 2012). For instance, on the dot-probe task ${ }^{1}$, when emotional face expressions were presented for $1000 \mathrm{~ms}$, depressed individuals, in comparison to non-depressed participants, displayed longer reaction times (reflecting an attentional bias) to sad but not happy, angry, or fearful faces (Donaldson et al., 2007; Gotlib et al., 2004). Interestingly, this negative attentional bias has also been observed in remitted depressed adults (Joorman \& Gotlib, 2007) as well as non-depressed girls who were at familial risk for depression (Joorman et al., 2007b; Kujawa et al., 2011), suggesting that attentional biases might be a marker or risk for onset and recurrence of depressive episodes. Consistent with these findings, in an eye-tracking experiment, depressed individuals spent more time looking at pictures featuring sadness and loss than non-depressed individuals (Eizenman et al., 2003). However, depressed individuals were no more likely than non-depressed individuals to shift their attention toward negative stimuli, but once their attention was focused on negative stimuli, they spent considerably more time looking at these stimuli than did non-depressed

\footnotetext{
${ }^{1}$ In the dot-probe task, a pair of stimuli (words or faces) is presented simultaneously (one stimulus is neutral and the other is emotional), and the individual is asked to respond to a probe that replaces either the neutral or the emotional stimulus. Allocation of attention to the spatial position of the stimulus is determined from response latencies to the probes (Gotlib \& Joorman, 2010).
} 
controls (Caseras et al., 2007; Kellough et al., 2008). It thus appears that depressed individuals might not automatically orient towards negative information, but once such information has captured their attention, they spend more time processing this information, possibly stemming from reduced cognitive control and inhibition (Gotlib \& Joorman, 2010; Joorman et al., 2007).

Several studies documented heightened amygdala activity among depressed individuals during the passive viewing of negative stimuli (Anand et al., 2005; Ditcher et al., 2009; Peluso et al., 2009; Siegle et al., 2002, 2007; Victor et al., 2010; but see Davidson et al., 2003; Irwin et al., 2004), which may reflect an attentional bias towards negative information (Foland-Ross \& Gotlib, 2012). Enhanced amygdala activity among depressed individuals also persists even after the removal of a negative stimulus (Siegle et al., 2002; 2007). Specifically, during presentation of personally relevant negative words depressed individuals displayed a sustained amygdala response that persisted for up to 30 seconds (Siegle et al., 2002), In fact, this activity was sustained even when depressed individuals engaged in a subsequent non-emotional distraction task (Sternberg memory task) designed to induce activation in brain regions (dorsolateral PFC) hypothesized to inhibit amygdala activity (Siegle et al., 2002). Given these findings, it appears that difficulties disengaging attention from negative emotional information may be rooted, or at least related to, persistent amygdala activation as well as difficulties of prefrontal regions to inhibit such activity, although this does not preclude the involvement of other cortical or limbic regions.

It was proposed that excessive processing of negative information might facilitate enhanced memory of negative, compared to positive life experiences (Mathews \& MacLeod 2005; Joorman, 2007). Consistent with this suggestion, depressed individuals were frequently reported to remember more negative information and less positive information, relative to non- 
depressed individuals (Bradley et al, 1995a, b; Dunbar \& Lishman, 1984; Direnfeld \& Roberts, 2006; Gur et al., 1992; Matt et al., 1992; Mogg et al., 1995; Ruiz-Caballero \& Bermudez, 1995; Rinck \& Becker, 2005). The amygdala is centrally involved in the encoding and retrieval of emotional material (Cahill et al., 1995; Adolphs et al., 1997), and this structure exerts a bottomup influence on other brain regions, including the hippocampus, which subserves episodic memory formation and retrieval (Steinvorth et al., 2005). Thus, neuroimaging studies that attempted to elucidate the neural underpinnings of negative memory biases for negative material have focused largely on activation of this region. For instance, following a sad mood induction, bilateral amygdala response during the encoding of emotional material predicted increased recall of negative (but not of positive) words in formerly depressed individuals (Ramel et al., 2007), but not among non-depressed individuals or in remitted depressed participants who had not received a sad mood induction. Increased memory sensitivity for negative material in depressed individuals was also associated with greater activity in the right amygdala during successful encoding of this material. Once more, this activation pattern was not present in non-depressed individuals during the encoding of negative stimuli, or in depressed individuals during the encoding of positive stimuli (Hamilton \& Gotlib, 2008). Furthermore, increased activation of the amygdala in depressed individuals was accompanied by increased functional connectivity between the amygdala and the hippocampus, caudate, and putamen, suggesting that during the encoding of negative material, depressed individuals engage a broader neural network that is involved, more generally, in enhancing memory for affective information (Hamilton \& Gotlib, 2008).

Appraisal and Interpretation 
Depressive disorders have been associated with negative appraisals and interpretive biases concerning personally meaningful life experiences (Abramson et al., 1989; Beck, 1967), including a tendency to attribute negative life events to internal (that they themselves are fundamentally flawed or worthless), stable (likely to persist over time), and global (likely to affect all aspect of life) causes (Abramson et al., 1989). Additionally, individuals with heightened depressive symptoms appraise potential stressors as relatively threatening (Folkman \& Lazarus, 1986).

The amygdala has been implicated in the automatic processing of biological significant stimuli, particularly those of threatening nature (Davis, 1994; Phelps \& Anderson, 1997). For example, electrical stimulation or abnormal activation of specific amygdala nuclei in rodents can produce a complex pattern of behavioural and autonomic changes that resemble a state of fear (Davis \& Whalen, 2001), whereas lesions to this site impair the acquisition and expression of conditioned fear responses (LeDoux, 2000). Functional neuroimaging studies with healthy individuals demonstrated that the amygdala responds to complex pictures of fearful and threatening scenes, such as snarling dogs, snakes, explosions, and attacks (Davis \& Whalen, 2001) as well as various facial stimuli, particularly those reflecting fear (Breiter et al., 1996; Morris et al., 1996), disgust (Phillips et al., 1997), sadness (Bliar et al., 1999) or anger (Hariri et al., 2002). In contrasts, humans with lesions to the amygdala display a deficit in the processing of fearful facial expression, whereas their processing of other facial expressions is less consistently compromised (Adolphs et al., 1995).,

Depressed individuals often exhibit over-activation of the amygdala (especially the left amygdala) in response to fearful or sad faces, even when these faces are presented for very short time periods $(<14 \mathrm{~ms})$, so that the individual was not consciously aware of ever seeing them ( $\mathrm{Fu}$ 
et al., 2004; Sheline et al., 2001). They also displayed increased amygdala activity in anticipation of negative, but not upon expecting neutral or positive stimuli (Abler et al., 2007). These findings are consistent with the heightened resting-state metabolic rate in the amygdala (Drevets et al., 1992). Given the role of the amygdala in the automatic processing of threatening and aversive stimuli, abnormal activity within this brain region may be associated with, or perhaps contribute, to the dysfunctional appraisals typically observed among depressive individuals. Importantly, appraisal of personally meaningful events is a continuous process, such that the initial appraisal may be followed by reappraisal (Lazarus, 1999; Gross, 2002). In this regard, the PFC has been implicated in effortful appraisals, and in particular the process of reappraisal (Ochsner \& Gross, 2005). Several neuroimaging studies demonstrated increased activation in predominately dorsal prefrontal and cingulate regions when individuals were instructed to reduce negative emotions by reappraising a negative situation, and this was further associated with attenuated amygdala and insular activity. These same prefrontal and cingulate regions were activated when individuals were asked to reappraise a situation in order to increase negative affect, although in this case, this form of reappraisal was accompanied by increased amygdala and insula activity (Ochsner \& Gross, 2005). Thus, it seems that the initial rapid appraisal and emotional responses elicited by a stimulus or situation might involve prefrontal regions which either inhibit or enhance the emotional responses that might be evoked by the amygdala.

Individuals with heightened depressive symptoms engage in reappraisal less frequently than non-depressed individuals (Garnefski \& Kraaj, 2006; Gross \& John, 2003), and these actions might be linked to abnormal activity within the dorsal aspects of the PFC and the cingulate. As described earlier, under resting conditions, depressed individuals typically exhibit attenuated activity within dorsolateral PFC and the dorsal ACC, coupled with heightened 
amygdala activity (Phillips et al., 2003). Furthermore, these individuals require greater recruitment of the dorsal PFC and ACC in order to perform to the level of non-depressed individuals on complex problem-solving tasks (Harvey et al., 2005). It may be that depressed individuals need to engage more prefrontal resources in order endorse effortful reappraisals, but this has, as yet, not been evaluated. It is conceivable that persistent utilization of these prefrontal resources, as in the context of chronic stressors, might progressively exhaust them, a cognitive form of allostatic overload, making reappraisal that much more difficult, and ultimately favoring the evolution and maintenance of depressive states.

\section{Coping and Emotion Regulation}

Depressive disorders have frequently been associated with the endorsement of inappropriate or effective coping strategies (Matheson \& Anisman, 2003; Nolen-Hoeksema, 2000). In particular, depressed individuals tend to engage in more emotion-focused coping (e.g., rumination and blame) and less frequent utilization of problem-focused coping methods, including problem-solving and cognitive restructuring (Matheson \& Anisman, 2003; Ravindran et al., 1999, 2002). The endorsement of problem-focused coping strategies might very well reflect flexible cognitive processing given that they involve searching for multiple or alternative solutions to a problem (i.e., problem-solving) and reinterpreting negative situations in terms of potentially positive experiences (i.e., cognitive restructuring). By contrast, certain emotionfocused coping strategies, especially rumination, might reflect cognitive inflexibility given that they involve stereotypical and perseverative thinking about the reasons for and meaning of one's own sad, dysphoric affect (Davis \& Nolen-Hoeksema, 2000; Nolen-Hoeksema et al., 2008). 
Although emotion-focused coping has generally been associated with negative outcomes, the effectiveness of certain coping strategies in mitigating distress or negative emotional responses may be context or situation dependent (Carver et al, 1989; Folkman \& Lazarus; 1985; Matheson \& Anisman, 2003). In emotionally charged situations, for example, emotional approach coping might be beneficial as it involves purposeful efforts to acknowledge and understand one's emotions, thus facilitating the individual's ability to come to terms with their feelings and might allow them to begin to let go (Stanton et al., 2000). Moreover, individuals typically do not use any particular coping strategy in isolation of others, and shifts from one strategy to another might occur as the situation demands (Matheson \& Anisman, 2003; Kelly et al., 2007). For instance, rumination can be combined with emotion-focused coping as well as with problem-focused methods (Kelly et al., 2007). Thus, cognitive flexibility can also be expressed as the ability to alternate between different coping strategies, including problem- and emotion-focused methods, according to changes in stressor demands or personal goals (Cheng, 2001; Matheson \& Anisman, 2003). Following from this notion, the persistent utilization of a narrow range of coping methods across different situations might favour the development of depressive states (Cheng, 2001, 2003; Matheson \& Anisman, 2003).

Several studies have attempted to elucidate the neural underpinnings of rumination, pointing to a network of brain regions, collectively referred to as the default mode network (DMN) (Berman et al., 2011; Cooney et al., 2010; Hamilton et al., 2011; Ray et al., 2005). The DMN, which is made up of the medial PFC, ventral-anterior and posterior cingulate, and inferior parietal regions, is typically activated when an individual is awake but not focused on a particular task or external environment (Raichle et al., 2001). Instead, this network is engaged during self-referential processing (Gunsward et al., 2001), which has been considered to be 
central process in rumination (Nejad et al., 2013). Using a rumination induction task, Cooney et al. (2010) examined individual differences in neural activity during rumination ("think about what people notice about your personality") versus concrete distraction ("think about a row of shampoo bottles on display") among depressed and non-depressed individuals. In comparison to non-depressed individuals, those who were depressed exhibited increased activation in the OFC, ventral ACC, and dorsolateral PFC during rumination versus concrete distraction. Additionally, during rumination versus abstract distraction neural activity was greater for depressed than for non-depressed individuals in the amygdala, rostral anterior cingulate/medial PFC, dorsolateral PFC, posterior cingulate, and para-hippocampus. Based on these findings, it was suggested that rumination might be associated with enhanced recruitment of limbic, medial and dorsolateral prefrontal regions in depressed relative to non-depressed individuals (Cooney et al., 2010). Importantly, rumination may be reflective (e.g., problem solving aimed at alleviating distress) or brooding (e.g., passively dwelling on the iniquity of one's current state) (Davis \& Nolen-Hoeksema, 2000). Reflective and brooding rumination are predictive, respectively, of lower and higher levels of depressive symptomatology years later, suggesting that reflective rumination is an adaptive cognitive process, whereas brooding is maladaptive (Treynor et al., 2003). Depressed individuals displayed more neural connectivity of the DMN, especially between the ventral and posterior cingulate cortex, compared to non-depressed individuals during rest periods, but this was not apparent during task engagement. Importantly, the restperiod connectivity correlated with self-report measures of depressive rumination and brooding, but not reflection (Berman et al., 2011). In line with these observations, among depressed individuals, higher levels of depressive rumination and lower levels of reflective rumination were associated with increased DMN dominance (Hamilton et al., 2011). 
During goal-oriented behaviour, the DMN is deactivated and another network, the taskpositive network (TPN), becomes engaged (Fox et al., 2005). The TPN, which is made up of the dorsal PFC and ACC, and the parietal cortex, governs several effortful cognitive processes, including cognitive control, attention, and working memory, and is typically activated during complex problem-solving task (Barch et al., 2003; Elliot et al., 1997; George et al., 1997; Harvey et al., 2005; Hugdahl et al., 2004; Okada et al., 2003; Videbech et al., 2003; 2004). From this perspective, the TPN is likely to be the network involved in problem-focused coping strategies. As previously noted, neuronal loss as well as hypo-functioning of several components of the TPN (e.g., dorsolateral PFC and dorsal ACC) have been observed among depressed individuals (Phillips et al., 2003), which might explain the under-utilization of problem-focused coping strategies (Matheson \& Anisman, 2003). Moreover, depressed individuals have been shown to require greater recruitment of several regions comprising the TPN in order to match the performance of non-depressed individuals (Harvey et al., 2005). Thus, depressed individuals may need to engage more TPN resources in order endorse problem-focused coping strategies. Furthermore, as in the case of stressor reappraisals, persistent utilization of the TPN, as might occur in the context of chronic stressors, might exhaust this network's resources (Harvey et al., 2005) so that DMN activation might predominate. This, in turn, would lead to a shift from problem-focused coping methods to emotion-focused coping, ultimately contributing to the evolution and maintenance of depressive illness. This view is in line with the increased DMN over TPN dominance observed among depressed individuals (Hamilton et al., 2011)

Finally, as previously mentioned, effective coping might involve the ability to alternate between coping strategies, including those geared towards problem-solving and those aimed at regulating emotions (Cheng, 2001; Matheson \& Anisman, 2003). From a neuroanatomical 
perspective, this might mean the ability to shift between the engagement of the TPN and the DMN. A brain region central to the switching between states of relative dominance of the DMN and TPN is the fronto-insular cortex (Sridharan et al., 2008). Thus, this might be a critical region involved in alternating between coping strategies. Interestingly, non-depressed individuals exhibited increased fronto-insular cortex response at the onset of increases in DMN activity, whereas depressed individuals displayed increased fronto-insular cortex at the onset of TPN (Hamilton et al., 2011). Given these findings, and the involvement of the DMN and TPN in emotion- and problem-focused coping, respectively, abnormal activity in the fronto-insular cortex might be a neural explanation for the excessive utilization of emotion-focused coping strategies at the expense of problem-oriented methods typically observed in depressed individuals (Matheson \& Anisman, 2003; Ravindran et al., 1999, 2002).

\section{Summary and Conclusion}

As outlined in the present review, cognitive flexibility plays a pivotal role in the ability to adapt to a continuously changing environment, and thus, has been considered as a hallmark of human cognition. Additionally, the available data suggest that cognitive flexibility involves multiple brain regions, but not surprisingly, prefrontal cortical areas are fundament for this ability. Although most studies that assessed cognitive flexibility were concerned with monoamine variations, it is likely that multiple neurochemical processes contribute in this regard. Given the importance of neurotrophic factors, such as BDNF, in both memory processes

and mood states, it is reasonable to propose further evaluation of growth factors in underlying the links between cognitive flexibility and mood states that are influenced by stressful experiences. There are limited data available assessing the link between particular genes and cognitive processes, although the data that available have pointed to the involvement of monoamine- 
related polymorphisms in cognitive flexibility. Even though gene x environment (stressor experiences) interactions were implicated in depressive disorders, comparable data have not been reported in relation to cognitive flexibility. Likewise, there has only been a single, inconclusive study concerning the relationship between BDNF and cognitive flexibility.

In addition to its importance in everyday situations, such as problem-solving, recent evidence has suggested that cognitive flexibility (and cognitive control processes in general) may play a pivotal role in the regulation of emotions. Indeed, disturbances in cognitive flexibility have frequently been associated with various mood and anxiety disorders, including depressive illness, and reduced cognitive control has been associated with increased use of rumination. Yet, little is known about how stressors might serve to impair or enhance this ability. To be sure, stressful events can have multiple effects on cognition, depending on the characteristics of the stressor, and cognitive flexibility is considered to be the manifestation of multiple cognitive processes. From this perspective, it may not be surprising that the effects of stressors on cognitive flexibility might vary with different situational demands and individual characteristics, including age, gender, and genetic predisposition. Certainly, more research is needed in order to understand how stressors affect cognitive flexibility, and the processes through which this occurs.

The present research set out to examine the relationship between different types of stressors (e.g., acute and chronic) and cognitive flexibility, as assessed through the WCST, and the cognitive and biological processes mediating these relationships. A second goal of the present research was to explore the ways through which cognitive flexibility (or inflexibility) might be expressed in stressful situations, and how this might, ultimately, be associated with symptoms of depression. 


\title{
CHAPTER 1
}

\section{The effects of an acute psychosocial stressor on cognitive flexibility: Relation to stressor appraisals and depressive symptoms}

\begin{abstract}
Acute stressors have frequently been shown to influence cognitive functioning, including learning and memory, but less is known about how cognitive flexibility is affected. The present investigation examined the effects of an acute psychosocial stressor (the Trier Social Stress Test; TSST) on a specific aspect of cognitive flexibility comprising set-shifting, as assessed through the Wisconsin Card Sorting Task (WCST). Among male and female undergraduate students $(N=$ 64), exposure to the TSST, which increased cortisol levels, was associated with fewer perseverative and non-perseverative errors as well as fewer trials to learn the initial sorting category. The enhancing effects of the TSST on set-shifting (fewer perseverative errors) were mediated by heightened threat appraisals. In the stressor condition (i.e., TSST), elevated symptoms of depression were associated with more perseverative and non-perseverative errors as well as more frequent failures to maintain an attentional set, and these relations were mediated by greater perceptions of uncontrollability. The present findings suggest that, by acting through the appraisal process, an acute stressor can linked to aspects of cognitive flexibility (attentional set-shifting), which might facilitate the disengagement of a previously, but no longer, effective behavioral response. Among depressed individuals, however, these stressor-elicited enhancements might not occur and instead individuals persist in the utilization of ineffective behavioral strategies.
\end{abstract}




\section{INTRODUCTION}

Stressful events can have a considerable impact on cognitive functioning, varying with the nature of the stressor encountered (Arsten, Raskind, Taylor, \& Connor, 2015; Lupien, Maheu, Tu, Fiocco, \& Schramek, 2007; McEwen \& Morrison, 2013). Generally, chronic stressors have consistently impaired a broad range of cognitive functions (Hurtubsie \& Howland, 2016; Marin et al., 2011; McEwen \& Morrison, 2013), whereas acute stressors had either enhancing and impairing effects depending on cognitive domain examined. For example, in the context of learning and memory, acute stressors facilitated encoding and consolidation processes, especially for threat-related or emotional information, but impaired retrieval of previously acquired information (Roozendraal, McEwen, Chattarji, 2009; Cahill, Gorski, \& Le, 2003; Kuhlmann, Piel, \& Wolf, 2005). Working memory can also be impaired following an acute stressor (Arsten \& Goldman-Rokic, 1998; Luethi, Meier, \& Sandi, 2009; Schoofs, Preuss, \& Wolf, 2008), although this effect might depend on characteristics of the stressor, such as its controllability (Arsten, 2009) as well as specific sub-processes of working memory (Hupbach \& Fieman, 2012; Schoofs, Wolf, \& Smeets, 2009; Stauble, Thompson, \& Morgan, 2013). It has been suggested that acute stressors, particularly those which are uncontrollable, can impair cognitive functions governed by the prefrontal cortex (PFC), while enhancing processes mediated by limbic regions (e.g., amygdala and basal ganglia) through changes of catecholamine and glucocorticoid activity (Arsten et al., 2015).

In comparison to learning and memory processes, less is known about the effects of acute stressors on cognitive flexibility, or the ability to modify cognitive content (e.g., "sets") and subsequent behavioral strategies in response to changes in environmental demands (Hurtubise \& Howland, 2017). In this regard, exposure to an acute psychosocial stressor (e.g., the Trier Social 
Stress Test; TSST) was accompanied by reduced performance on several measures thought to reflect cognitive flexibility, including solving anagrams and a compound remote associates task (Alexander et al., 2007) as well as multi-tasking (Plessow et al., 2012). It is possible that acute stressors, through neurochemical or hormonal (e.g., cortisol) changes induced, have divergent effects on tasks that assess various aspects of cognitive flexibility. Accordingly, the primary goal of the present study was to examine the effects of an acute psychosocial stressor on a specific aspect of cognitive flexibility, namely attentional set shifting, as assessed by the Wisconsin Card Sorting Task (WCST; Grant \& Berg, 1948; Heaton, Chelune, Talley, Kay, \& Curtiss, 1993). Furthermore, as changes of cortisol levels have been associated with prefrontal cognitive functions (Lupien et al., 2007), we hypothesized that the effects of an acute stressor on cognitive flexibility would be mediated by cortisol elevations.

The characteristics of the stressor might contribute to whether an individual engages in flexible or inflexible cognitive and behavioral responses (Arsten et al., 2015). When faced with a stressful event, perceptions of threat might mobilize and allocate cognitive resources in order to contend with a stressor and, if the stressor is perceived as being controllable, then PFC-governed cognitive functions, such as cognitive flexibility, might be enhanced. However, if the stressor is perceived as uncontrollable, PFC-mediated cognitive abilities may become impaired, thereby contributing to a reduction in cognitive flexibility, so that relatively automatic and inflexible responses predominate. In essence, uncontrollable stressors might favor the endorsement of reflexive or habitual (automatic) responses, whereas controllable stressors might favor more flexible reactions (Arsten et al., 2015). Accordingly, we assessed whether stressor appraisals related to threat and control mediated the effects of an acute stressor on WCST performance. 
Finally, depressed individuals have frequently been shown to exhibit disturbances in cognitive functioning, especially cognitive flexibility (Fossati, Ergis, \& Allilaire, 2001; Snyder, 2013; Trivedi \& Greer, 2014). However, attentional set-shifting performance amongst individuals expressing depressive features has not been examined following an acute stressor. Thus, another central aim of the present study was to examine the relationship between depressive symptoms and WCST performance following an acute stressor, and to determine whether this relation was mediated by perceptions of threat, control, and affective state.

\section{METHODS}

\section{Participants}

Initially, 80 participants were recruited from an online computerized recruitment system used by the university. From this initial sample, 16 individuals were excluded due to the use of anti-depressants and/or psychostimulants (e.g., methylphenidate), presence of a neurological disorder or learning disability. The final participant sample comprised 64 (female: $n=44$ ) Carleton University undergraduate students. Participant age ranged from 17 to $25(M=19.26$, $S D=1.91)$ and reported ethnicities included White $(52.3 \%, n=34)$, Black $(23.1 \%, n=15)$, Arab/West Asian (9.2\%, $n=6)$, South Asian (4.6\%, $n=3)$, Latin American/Hispanic $(3.1 \%, n=$ $2)$, Asian $(1.5 \%, n=1)$, and other $(6.2 \%, n=4)$.

\section{Procedure}

All procedures in this study were approved by the Carleton University Ethics Committee for Psychological Research. Laboratory sessions were conducted between 1300 and 1730 hr, and participants were asked not to eat, drink (with the exception of water) or smoke for at least an hour before arriving to the session. Once informed consent was signed, participants filled out several questionnaires assessing demographic information (e.g., age and gender), general health 
(e.g., neurological disorders) and medication history (e.g., psychostimulants), and depressive symptomatology. This allowed participants 30 minutes to habituate to the laboratory environment.

Following the 30-minute relaxation period, participants were randomly assigned to either the stressor or control condition. In the stressor condition, participants were instructed that they would be given 5 minutes to prepare for an employment task comprising a five-minute speech and five-minute mental arithmetic task in front of a panel of graduate student judges. In addition, participants were told they were being videotaped during the psychosocial stressor. Immediately following the 5-minute preparatory period, participants in the stressor condition underwent the TSST.

Participants in the control condition were asked to complete an employment task, which comprised writing down their strengths and past work/volunteer experience on a form. Following the TSST or control task, participants were given 5 minutes to complete two questionnaires concerning measures of stressor appraisal and affect scores. At 5 minutes, participants completed a computerized version of the WCST. As the study was part of a larger effort to define factors that promote resilience, in addition to the WCST, participants also completed other tasks (e.g., a decision-making task and an implicit learning task), which were administered in a counterbalanced manner. Once these tasks were completed, which required approximately 20 to 25 minutes, participants completed a questionnaire (21-item Beck Depression Inventory) measuring depressive symptoms and were then fully debriefed concerning the purpose of the present study. 


\section{Cognitive Flexibility: Attentional Set-Shifting}

Cognitive flexibility, assessed through attentional set-shifting was determined using a computerized version of the WCST, referred to as Berg's Card Sorting Task (BCST), provided by the Psychology Experiment Building Language (PEBL) version 0.14 (Mueller \& Piper, 2014). The BCST consists of a 128-card deck with each card containing a different combination of one of four shapes, colors, and quantities. Four key cards are displayed at the top of the screen as a guide to help determine which of the four stacks the deck's up-card is sorted to. The deck is revealed one card at a time, and the visible card is matched to key cards depending on the particular rule (unknown to the participant) for a given set. After ten cards have been successfully matched, the set is completed and the sorting rule changes (also unknown to the participant). The new rule must be discovered using trial and error via feedback received after each card is sorted. After a card is sorted, the participant is provided with feedback regarding whether it was sorted correctly (i.e., according to the current rule). This process continues until the participant either sorts all 128 cards, or until the participant successfully completes 9 sets/categories, whichever comes first (for more information see Fox et al., 2013). The BCST takes approximately 10 minutes to complete.

The BCST assesses several performance measures, including the number of categories experienced (from 1 to 9 ) and categories completed (from 0 to 9). The primary measures of the $\mathrm{BCST}$ are the type of errors the individual makes. Perseverative errors occur when the individual continues to sort cards according to a previously, but no longer, relevant or correct sorting rule. These types of errors were the central measure of reduced cognitive flexibility, attentional set-shifting. In the present study, perseverative errors were computed according to procedures described by Heaton et al. (1993). Non-perseverative errors, by contrast, refer to all 
other errors. Among the non-perseverative errors are failures to maintain set, which refers to selecting an incorrect card once a sorting rule has been learned (i.e., switching after the fifth correctly sorted card). Failures to maintain a set are thought to represent distractibility or difficulties maintaining information online in working memory (Barcelo \& Knight, 2002). Finally, the BCST also assesses trials to first category, which refers to the speed of which an individual acquires an attentional set.

\section{Salivary Cortisol}

Saliva samples were collected in Salivette tubes, (Sarstedt, Germany), 30 mins after participant's arrival to the laboratory (baseline) as well as 5, 15, 30, and 45 mins following the TSST or control condition. Immediately following the experimental session, saliva samples were frozen at $-80^{\circ} \mathrm{C}$. Following the manufacturer's protocol, a competitive radioimmunoassay, 125I kit (ICN Biomedicals Inc., Irvine, CA), was used to determine, in duplicate, salivary cortisol levels. The intra- and inter-assay variability was $<10 \%$. The minimum detectable level of cortisol was $0.02 \mu \mathrm{g} / \mathrm{dl}$ and the specificity was $100 \%$ cortisol. One participant did not have five valid cortisol measures and thus were appropriately removed from the repeated measures and AUCi analysis.

\section{Measures}

Stressor appraisals. A shortened version of the Stress Appraisal Measure (SAM; Peacock and Wong, 1990) was used to assess three dimensions of stressor appraisals immediately following the TSST (or control task). In particular, this short version included 12 items concerning perceptions of threat (“How threatening was this situation?"), uncontrollability ("Was this a totally hopeless situation?" "Was the outcome of this situation uncontrollable by anyone?"), and control-by-self ("Did you have the ability to do well in this situation?", "Did you 
have the skills necessary to achieve a successful outcome to this situation?'). Scores for each appraisal dimension were computed by taking the mean across all four relevant items. These items were measured on a five-point scale ranging from 1 (not at all) to 5 (extremely), with higher scores indicating higher levels of perceived threat $(\alpha=.88)$, control-by-self $(\alpha=.75)$, or uncontrollability $(\alpha=.70)$

Mood. The 20-item Positive and Negative Affect Schedule (PANAS; Watson, Clark, \& Tellegen, 1988) was used to assess positive and negative affect post stressor. Responses ranged on a six-point scale from 1 (very slightly or not at all) to 5 (extremely). Positive and negative affect scores were computed by summing across all 10 relevant items for each subscale. Cronbach's alphas were: positive (.85) and negative (.90).

Depression. The 21-item Beck Depression Inventory (BDI) was used as a measure of depressive symptomatology (Beck, Ward, \& Mendelson, 1961). Each item comprises one of four options, ranging from low to high depressive symptomatology. Total scores were calculated by summing across all items $(\alpha=.88)$.

Statistical Analyses

Statistical analyses were performed using SPSS for Windows 18.0 (SPSS Science, Chicago, IL). A MANOVA was used to determine the effects of the TSST condition on stressor appraisals, including perceived threat, uncontrollability, and control-by-self, followed by an independent-sample t-test was used to examine differences between the stressor and control group on depressive scores. A MANOVA was also used to examine the effects of the TSST on positive and negative mood.

Cortisol changes over time as a function of stressor condition were analyzed using a 2 (Stressor Condition) x 5 (Time: 5 time-points) mixed measures ANOVA with Time serving as 
the within-group factor. Follow-up comparisons comprised t-tests with a Bonferroni correction to maintain the alpha level at 0.05. A MANOVA was conducted to examine differences between the stressor and control groups on performance indices of the WSCT, including categories experienced, categories completed, perseverative errors, non-perseverative errors, failures to maintain set, and trials to first category. In addition, area under the curve (AUCi) was computed in order to examine changes of cortisol in relation to the WCST (Pruessner, Kirshbaum, Meinlschmid, \& Hellhammer, 2003).

Multiple linear regression analyses were used to examine the unique contribution of specific stressor appraisals on perseverative errors, non-perseverative errors, failures to maintain set, and trials to first category (threat, uncontrollability, and control-by-self appraisals were entered as predictors simultaneously). Mediation analyses were conducted using the PROCESS add-on to SPSS provided by Preacher \& Hayes (Preacher \& Hayes, 2008; Hayes, 2013), using 5000 bootstrap samples and 95\% Bias Corrected Confidence Intervals (C.I.). A multiple mediation analysis was used to examine mediating effects of threat and uncontrollability in the relation between stressor condition and perseverative errors. Multiple linear regression analyses, in which positive and negative affect were entered in as predictors simultaneously, was used to examine the relation between mood and perseverative, non-perseverative errors, failures to maintain set, and trials to figure category.

Pearson's correlation coefficients were determined between cortisol AUCi and WCST performance indices. A simple mediation analysis was used to examine whether cortisol AUCi mediated the relation between stressor condition and perseverative errors on the WCST. Hierarchical linear regression analyses were implemented to examine the moderating role of depressive symptoms in the relation between stressor condition and WCST performance indices. 
Pearson's correlation coefficients were determined between symptoms of depression and WCST performance indices as well as stressor appraisals, affect, and cortisol AUCi in the control as well as stressor condition. Multiple mediation analyses served to examine the mediating role of threat and uncontrollability in the relation between depressive symptoms and WCST performance. Finally, simple mediation analyses were implemented to examine the mediating role of negative affect in the relation between depressive symptoms and WCST performance.

\section{RESULTS}

Acute stressor effects on WCST performance

A MANOVA revealed no significant group differences in the number of categories experienced in the WCST, $F(1,62)=.33, p>.05$, or the number of categories completed, $F(1$, $62)=.39, p>05$. However, individuals in the stressor condition made significantly fewer perseverative errors, $F(1,62)=4.09, p<.05$ and non-perseverative errors, $F(1,62)=4.15, p<$ .05 (Figure 1A). They also learned the first sorting category quicker, $F(1,62)=4.57, p<.05$ (Figure 1B), but group differences were not observed in the ability to maintain an attentional set, $F(1,62)=.82, p>.05$ (Figure 1C). 

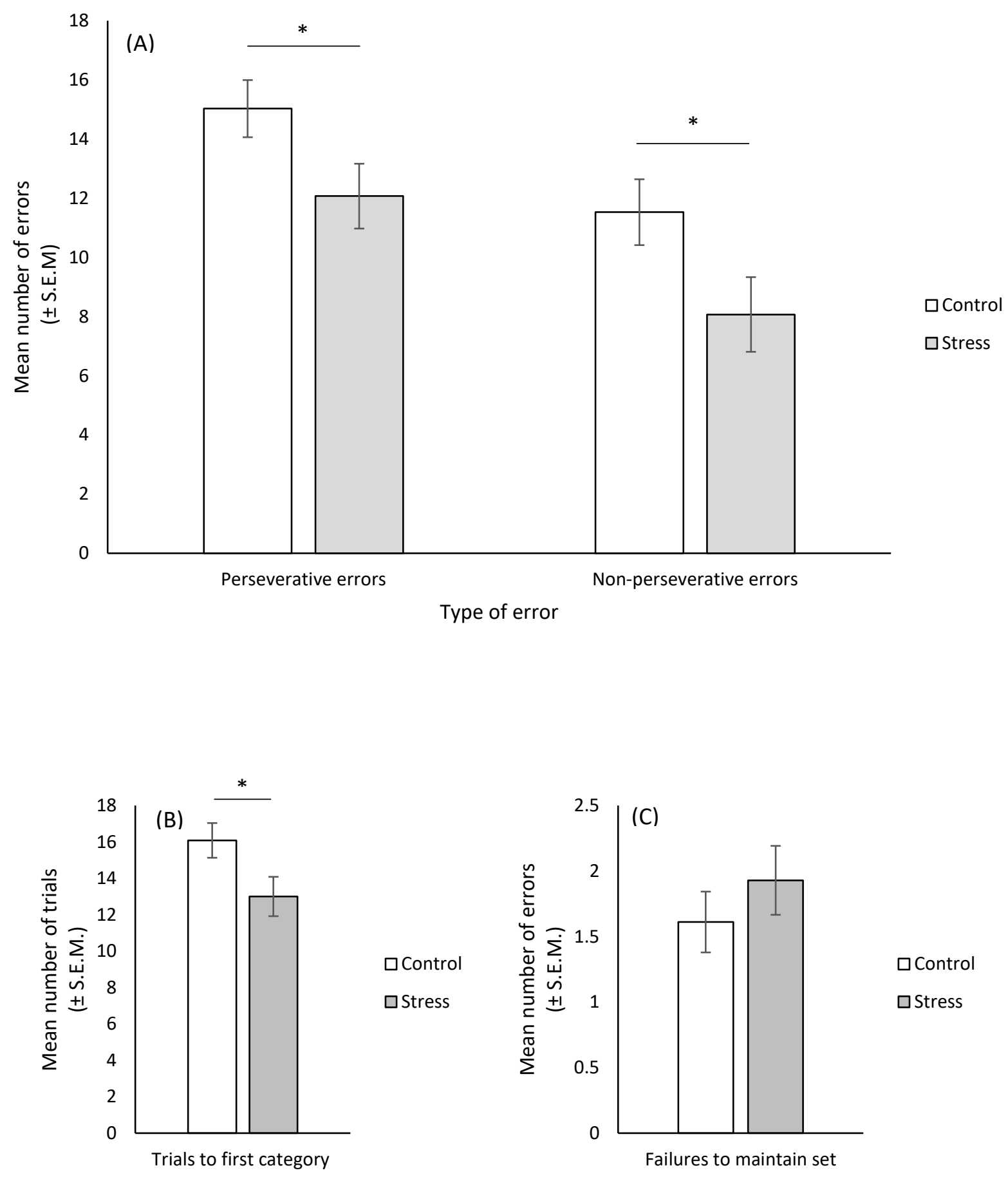

Figure 1. Differences $( \pm$ S.E.M.) in (a) perseverative versus non-perseverative errors, (b) trials to first category, and (c) failures to maintain an attentional set among individuals in the stressor and control conditions. Note $* \mathrm{p}<.05$ 


\section{Relation between cortisol levels and WCST performance}

Results of a mixed measures ANOVA revealed a significant effect of Time, $F(4,59)=$ 9.347, $p<.001$, as well as a Stressor Condition $\mathrm{x}$ Time interaction, $F(4,59)=8.20, p<.001$. As illustrated in Figure 2, and confirmed by follow-up tests of the simple effects comprising this interaction, individuals in the stressor condition exhibited elevated cortisol levels 5, 15, 30, and 45 minutes following the TSST compared to those who were in the control condition. Moreover, relative to the control condition $(M=-10.59, S D=28.70)$, a larger cortisol AUCi $(M=14.25, S D$ $=26.48)$ was apparent in the stressor condition, $t(62)=-3.55, p<.001$.

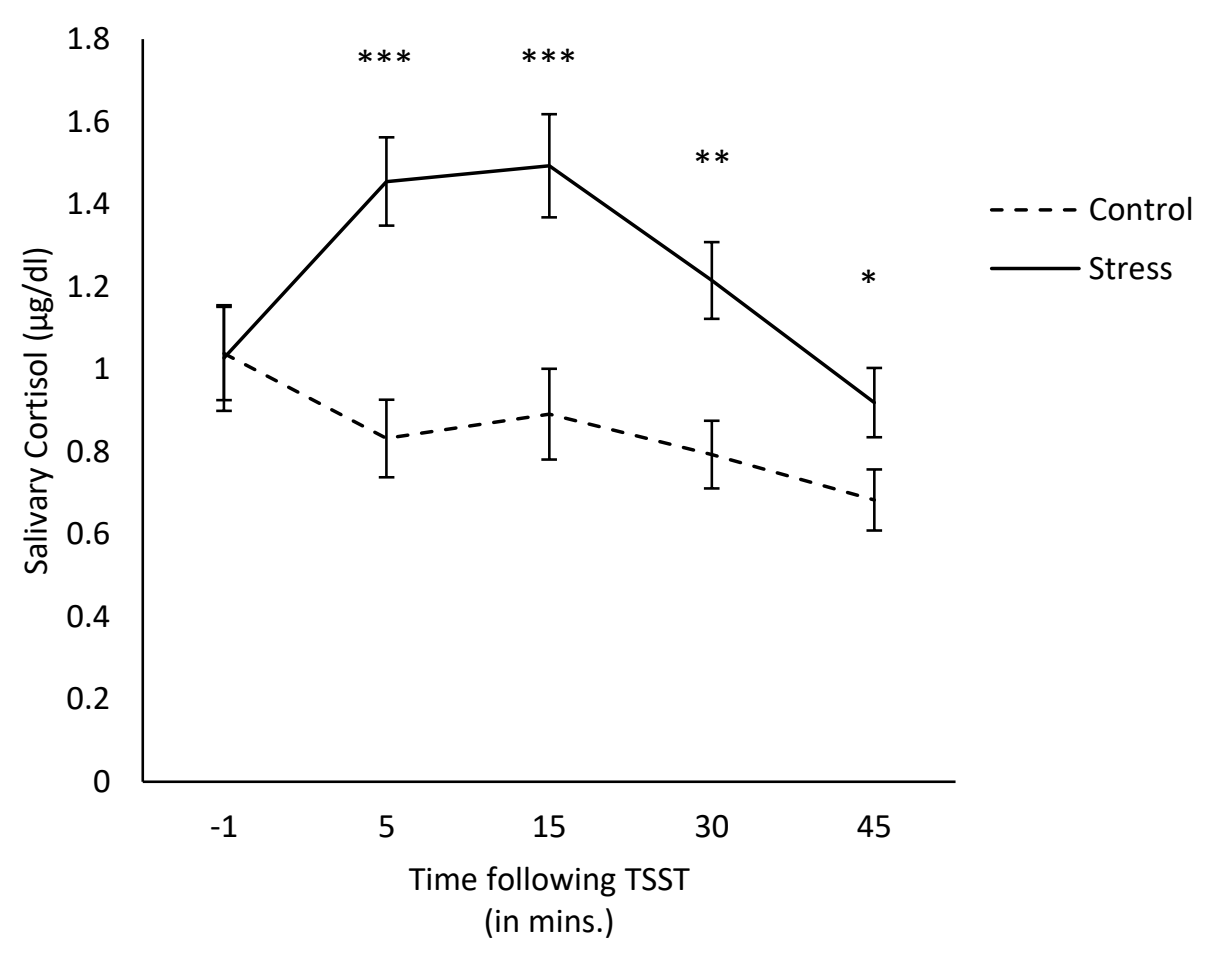

Figure 2. Salivary cortisol levels $( \pm$ S.E.M. $)$ over time between individuals in the stressor and control conditions. Note: ${ }^{* * *} p<.001,{ }^{* *} p<.01,{ }^{*} p<.05$. 
Next, we examined zero-order correlations between cortisol AUCi and performance measures of the WCST. Greater cortisol AUCi was associated with fewer perseverative errors $(r$ $=-.26, p<.05)$, but AUCi did not significantly mediate the relation between stressor condition and perseverative errors $(95 \%$ C.I.: $-2.64, .44)$. Furthermore, cortisol AUCi was unrelated to non-perseverative errors $(r=-.20, p>.05)$, failures to maintain a set $(r=-.01, p>.05)$, or number of trials required to learn the first category $(r=-.20, p>.05)$.

The mediating role of stressor appraisals in the relation between an acute stressor and WCST performance

As expected, the stressor (i.e., TSST) was appraised as being more threatening, $F(1,63)$ $=18.94, p<.001$, and there was a trend for individuals to perceive the stressor condition as more uncontrollable, $F(1,63)=3.47, p=.07$. Perceptions of control-by-self, $F(1,63)=.05, p>.05$, by contrast, did not differ between the stressor and control conditions. Given these findings, it was of interest to determine whether performance on the WCST was associated with the way in which the stressor (or control condition) was appraised.

A multiple linear regression analysis revealed that when the three appraisal dimensions were considered (i.e., threat, uncontrollability, and control-by-self), only perceptions of threat and uncontrollability accounted for unique variance in perseverative errors on the WCST. In particular, greater perceived threat was associated with fewer perseverative errors $(b=-1.74$, S.E. $=.80, p<.05)$, whereas greater perceived uncontrollability was related to more perseverative errors $(b=2.38, S . E .=1.13, p<.05)$. A multiple mediation analysis revealed that only perceptions of threat (but not uncontrollability) significantly mediated the relation between stressor condition and perseverative errors (95\% C.I.: $-2.85,-.03)$. Specifically, compared to the 
control condition, the stressor condition was appraised as being more threatening, and this, in turn, was related to fewer perseverative errors. If fact, these relations were unique to perseverative errors as, according to several additional multiple regression analyses, threat and uncontrollability were not related non-perseverative errors, failures to maintain set, or trials to first category $(p ’ s>.05)$.

\section{Relation between mood and WCST performance}

As expected, individuals in the stressor condition reported significantly greater negative mood, $F(1,63)=36.79, p<.001$, but did not differ in positive mood, $F(1,63)=.23, p>.05$. Results of several multiple regression analyses indicated that positive and/or negative mood were not related to perseverative errors, non-perseverative errors, or trials to first category ( $p$ 's $>.05)$. However, greater negative mood $(b=.40, S . E .=.19, p=.05)$, but not positive $\operatorname{mood}(b=-.20$, S.E. $=.23, p>.05)$, was associated more frequent failures to maintain an attentional set.

Moreover, the relation between stressor condition and more frequent failures to maintain a set was mediated by heightened negative affect $(95 \%$ C.I.: .13, 1.23).

\section{Relation between depressive symptoms and WCST performance}

Depressed individuals have frequently been shown to exhibit disturbances in cognitive functioning, particularly cognitive flexibility (Trivedi \& Greer, 2014). It was, therefore, of interest to determine whether the effects of an acute stressor on the WCST performance would be moderated by symptoms of depression. Hierarchical linear regression analyses, however, indicated that depressive symptoms did not significantly moderate the effects of the stressor on any of the performance indices of the WCST.

Despite the absence of significant moderating effects, we examined the relation between depressive symptoms and performance indices of the WCST in the stressor $(n=28)$ and control 
$(n=37)$ conditions separately. In the control condition, depressive symptoms were not associated with perseverative errors $(r=.11, p=.52)$, failures to maintain an attentional set $(r=$ $.27, p=.12)$, or trials to first category $(r=.09, p=.60)$, although there was a moderate correlation between depressive symptoms and non-perseverative errors $(r=.31, p=.06)$. In the stressor condition, by contrast, heightened depressive symptoms were related to more failures to maintain an attentional set $(r=.47, p=.01)$ and, although not quite reaching the .05 alpha level of statistical significance, more perseverative $(r=.36, p=.06)$ and non-perseverative errors $(r=$ $.35, p=.07)$.

Given the relationship between depressive symptoms and performance on the WCST in the stressor condition, we examined potential mediators in linked to appraisals. In the stressor condition, heightened depressive symptoms were associated with greater perceived threat $(r=$ $.53, p<.01)$ and uncontrollability $(r=.46, p=.01)$, but not perceptions of control-by-self $(r=-$ $.21, p>.05)$. Additionally, depressive symptoms were related to negative affect $(r=.52, p<.01)$ but not positive affect ( $r=-.23, p>.05)$, and symptoms of depression were not related to cortisol $\operatorname{AUCi}(r=-.27, p>.05)$.

Following these analyses, we examined whether, in the stressor condition, the relation between depressive symptoms and performance outcomes on the WCST were mediated by perceptions of threat and uncontrollability as well as negative affect. As indicated by several multiple mediation analyses, uncontrollability significantly mediated the relationship between depressive symptoms and perseverative errors $(95 \%$ C.I.: .02, .30) as well as non-perseverative errors $(95 \%$ C.I.: .02, .32). Specifically, heightened levels of depression were associated with greater perceived uncontrollability, which was related to more perseverative as well as none perseverative errors. As well, uncontrollability mediated the relationship between depressive 
symptoms and failures to maintain an attentional set $(95 \%$ C.I.: .00, .11). In particular, elevated depressive symptoms were associated greater perceived uncontrollability concerning the stressor (i.e., the TSST), and this in turn was related to more failures to maintain a set. In contrast to these findings, negative affect did significantly mediate the relationship between depressive symptoms and WCST outcomes $(p$ 's $>.05)$.

\section{DISCUSSION}

Acute stressor effects on cognitive flexibility

The purpose of the present study was to examine the effects of an acute stressor on a specific type of cognitive flexibility, namely attentional set-shifting. As indicated by the present findings, exposure to an acute psychosocial stressor enhanced attentional set-shifting on the WCST, such that individuals more readily disengaged from previously, but no longer, relevant (or appropriate) information and behavioral responses. These stressor effects, however, were not limited to attentional set-shifting, as individuals in the stressor condition generally made fewer errors, including non-perseverative errors. Furthermore, stressor exposure was accompanied by more rapid acquisition of the initial attentional set, which might have partly contributed to the overall enhanced performance on the WCST among individuals in the stressor condition. However, performance on the WCST, and attentional set-shifting in particular, may be dependent on multiple cognitive processes which operate in parallel or sequentially, including attentional control, working memory, performance and error monitoring, and response selection and inhibition (Konishi et al., 1999a,b; Monchi et al., 2001). Thus, it is difficult to identify through which specific cognitive processes the stressor came to produce enhanced attentional set-shifting. 
In the present investigation, stressor effects on working memory, which was assessed by the number of times individuals failed to maintain an attentional set, was not observed. This may have been due to a floor effect as individuals in both the stressor and control conditions made relatively few errors specific to failures to maintain set (approximately 1 to 2). Although this may seem contrary to some previous findings (Luethi et al., 2009; Schoofs et al., 2008), it is important to consider that the effects of an acute stressor could potentially be dependent upon the specific aspects of working memory being assessed (Hupbach \& Fieman, 2012; Stauble et al., 2013). In the present investigation, as previously reported (Shoofs et al., 2009), exposure to an acute stressor had no effect on the "maintenance" of relevant information in working memory. Although the WCST does not allow for direct measurement of the "updating" component of working memory, this is believed to be an important process in WCST performance (Konishi et al., 1999a). Accordingly, it is possible that the enhancement in attentional set-shifting following the acute stressor might have been facilitated by increased speed in updating working memory content. Collectively, these data suggest that exposure to a moderate acute stressor can enhance cognitive processes (e.g., attention and working memory) that might facilitate adaptation to changes in the environment. In the context of the present study, this was ultimately manifested as the increased ability to disengage from previously, but no longer, appropriate behavioral responses and a shift towards newly effective response strategies.

These findings might seem contradictory to earlier studies that indicated that a stressor in the form of the TSST was associated with diminished performance on several measures of cognitive flexibility (Alexander, 2007; Plessow et al., 2012). As previously discussed, however, cognitive flexibility can be manifested in several ways, and each aspect of this ability might involve different underlying cognitive processes. It is, therefore, possible that even similar 
stressors could have distinct effects on different forms of cognitive flexibility. Specifically, it has been reported that exposure to the TSST was accompanied by disturbed performance on a remote associates test and solving anagrams (Alexander et al., 2007), which might reflect cognitive processes involved in creativity (Mednick, 1968). The behavioral task utilized in the present study (i.e., WCST), by contrast, more directly assesses an aspect of executive functioning involved in the ability to shift attention between multiple sources of information according to changes in task contingencies (Grant \& Berg, 1948; Konishi et al., 1998; Monchi et al., 2001). In essence, exposure to an acute stressor might enhance particular processes (e.g., attention and working memory) involved in cognitive flexibility, but could potentially still impair other features of flexibility, including creativity (Alexander, 2007; Byron, Khazanchi, \& Nazarian, 2010).

Exposure to the TSST reportedly disturbed performance in a task-switching paradigm (Plessow et al., 2012). In task-switching paradigms, the central measures are "switch costs", which refer to the difference in performance (i.e., response times and percentage of errors) on trials where individuals switch from one task to another relative to trials in which the same task is performed (Monsell, 2003). In the WCST, as mentioned earlier, the central performance measure is the frequency of perseverative errors, reflected by the number of times the individual continues to sort according to a previously, but no longer, correct sorting rule. Acute stressors might, therefore, facilitate cognitive processes necessary to disengage from no longer relevant information, but temporarily impair processes required for efficient multi-tasking, such as the reconfiguration and implementation of newly relevant task requirements (Plessow et al., 2012). 


\section{Relationship between cortisol and cognitive flexibility}

Acute stressors are typically accompanied by relatively immediate elevations in catecholamines (i.e., dopamine and norepinephrine), followed by the release of glucocorticoids (e.g., cortisol in humans), and it is through these neurochemical changes that stressors can influence PFC-dependent cognitive functions (Arsten et al., 2015; McEwen, 2013; Lupien, 2007). However, the impact of catecholamine and glucocorticoid release on PFC-dependent cognitive functions appear to follow an inverted-U shape. Specifically, whereas cognitive functioning is generally impaired by excessive release of these neurochemicals, relatively moderate elevations of catecholamine and glucocorticoid release can have enhancing effects (Artsen et al, 2015; Cools \& D'Esposito, 2011; Lupien, 2007).

In the present investigation, individuals in the stressor condition exhibited the expected elevations and subsequent decrease of cortisol levels, whereas those in the control condition displayed the typical cortisol decline over overtime. Interestingly, it was observed that greater cortisol AUCi was specifically associated with enhanced attentional set-shifting (i.e., fewer perseverative errors), but not the acquisition or maintenance of an attentional set. Given these findings, it might be that relatively moderate elevations of cortisol enhanced prefrontal cognitive functions which, in the context of the present study, was reflected through the ability to disengage from no longer relevant (or appropriate) information and behavioral responses.

Cortisol levels, however, did not mediate the relation between stressor condition and attentional set-shifting. It is possible that the stressor was accompanied by other neurochemical changes that had a more direct effect on the prefrontal activity, which then facilitated the enhancement in attentional set-shifting. As discussed earlier, in addition to glucocorticoids, the 
effects of acute stressors of PFC-dependent cognitive functions, might be mediated by catecholamine changes (Arsten et al., 2015).

Stressor appraisals and cognitive flexibility

Acute stressors, especially if they are uncontrollable, can favor a shift from top-down to bottom-up processing, which is subsequently accompanied by greater engagement in habitual, over flexible, behavioral responses (Arsten et al., 2015). We, therefore, examined whether the way in which the stressor was appraised would be associated with varying levels in attentional set-shifting performance. As expected, the TSST was appraised as more threatening, but was not considered particularly uncontrollable. Furthermore, individuals in the TSST and control conditions did not differ in their perceived ability to do well (i.e., control-by-self), which might suggest that the TSST did not overly tax individual's coping resources.

Interestingly, appraisals concerning threat and uncontrollability were specifically associated with attentional set-shifting, but not with the acquisition or maintenance of an attentional set. In particular, heightened threat was associated with enhanced attentional setshifting, whereas greater perceived uncontrollability was accompanied by reduced set-shifting. Accordingly, heightened perceived threat might serve to direct cognitive resources (e.g., attention) towards information that may be relevant to resolving a stressor, whereas perceptions of control contribute to the subsequent behavioral response. Specifically, situations perceived as uncontrollable might be accompanied by difficulties disengaging from a previously, but no longer, effective behavioral response. Situations perceived as controllable, by contrast, might be associated with increased adaptability to changing environmental demands.

Although threat and uncontrollability were related to attentional set-shifting, only perceptions of threat mediated the effects of the stressor on set-shifting. Specifically, the TSST 
was accompanied by heightened perceived threat, which, in turn, was associated with enhanced attentional set-shifting. Although perceptions of uncontrollability did not mediate the effects of the stressor on attentional set-shifting, this does not lessen the significance of this appraisal dimension. Indeed, the lack of mediating effects might have been due to the fact that the TSST was not perceived as particularly uncontrollable. Additionally, the present findings suggest that the influence of appraisal processes on attentional set-shifting might not be limited to stressful situations.

\section{Depressive Symptoms and cognitive flexibility}

Disturbances in cognitive flexibility have frequently been aligned with psychopathology, including depressive disorders (Trivedi \& Greer, 2014). Depressed individuals, especially those who were severely depressed (e.g., melancholic) or elderly (Austin et al., 1999), exhibited difficulties shifting their attention away from a previously, but no longer, appropriate behavioral responses. In contrast, in the present investigation, depressive symptoms were largely unrelated to performance on the WCST under basal (control) conditions. Interestingly, however, following an acute stressor, heightened depressive symptoms were associated with more frequent perseverative and non-perseverative errors as well as more failures to maintain an attentional set, and these relationships were mediated by greater perceived uncontrollability. Thus, through greater perceived uncontrollability, depressed individuals might fail to allocate the cognitive resources (attention and working memory) necessary to modify behavioral strategies according to changing environmental (e.g., task) demands.

Although, in the present study, it is not entirely clear how perceptions of uncontrollability among depressed individuals might have been associated with diminished attentional and working memory processes; however, uncontrollable stressors have been associated with 
impairments in prefrontal cognitive functions (Arsten et al., 2015). Thus, it is possible that operating through increased perceptions of uncontrollability, depressed individuals fail to recruit brain regions (e.g., prefrontal, anterior cingulate, and parietal cortices) involved in attentional set-shifting (Monchi et al., 2001), which might ultimately have contributed to the persistent engagement in ineffective or inappropriate behavioral strategies.

\section{Limitations and conclusions}

In considering the implications of the present study, several factors need to be considered. In this regard, certain aspects of cognition, such as executive functioning, tends to decline with increasing age (Deary et al., 2009). Moreover, stressors can have different effects on cognition across the lifespan (McEwen \& Morrison, 2013). As such, it is uncertain whether the enhancing effects of an acute stressor on attentional set-shifting reported in the presented study (which comprised relatively young individuals) would be apparent among older individuals. As well, participants in the present study comprised relatively few males and thus gender comparisons, particularly concerning the effects of cortisol changes on attentional set-shifting, were not possible. Finally, depressive severity was assessed using a self-report questionnaire and, while a fair number of individuals reported relatively moderate to high depressive symptoms, the majority of individuals were not necessarily depressed. Nonetheless, it is interesting that, when challenged, certain cognitive impairments were unmasked even among relatively mild to moderately depressed individuals.

Despite these limitations, the present findings suggest that exposure to an acute stressor can have enhancing effects on certain cognitive processes necessary in order to adapt to changing environmental demands, and that the stressor appraisal processes plays an important role in this regard. Among depressed individuals, however, exposure to an acute stressor might serve to 
impair cognitive functioning, resulting in the persistent utilization of ineffective behavioral strategies. Taken together, the present findings might provide further insight into the ways depressed and non-depressed individuals might cope with stressful life events. 


\title{
CHAPTER 2
}

\section{Traumatic life events and cognitive flexibility: moderating role of sex and the BDNF Val66Met gene polymorphism}

\begin{abstract}
Disturbances of cognitive flexibility have frequently been associated with psychiatric disorders, including depressive illness, yet the factors which might contribute to such disturbances are not fully understood. The present investigation examined whether a history of different forms of traumatic events was related reduced cognitive flexibility, as assessed by the Wisconsin Card Sorting Task (WCST), and whether the relationship between moderated by a BDNF polymorphism, sex, or a combination of both variables. Among male and female undergraduate students $(N=239)$, the relationship between trauma type and perseverative errors (i.e., measure of cognitive flexibility) on the WCST was moderated by both BDNF genotype and gender. Specifically, for both males and females, greater frequency of general traumas was related to more frequent perseverative errors among individuals carrying the Met allele of the BDNF polymorphism, but not among those who were $\mathrm{Val}$ homozygotes. Moreover, greater frequency of physical punishment was associated with more frequent perseverative errors only among males carrying the Met allele, whereas greater frequency of emotional abuse was related to more frequent perseverative errors among females carrying the Met allele of the BDNF gene polymorphism. The present findings that the relationship between traumatic life events and disturbed cognitive flexibility might depend on a combination of type of trauma, sex, and BDNF genotype.
\end{abstract}




\section{INTRODUCTION}

Cognitive flexibility refers to the ability to modify cognitive (attentional) sets or behavioral strategies in response to changing environmental demands. At the most fundamental level, this ability is the manifestation of several cognitive (executive) control processes which operate sequentially or in parallel, including attention, conflict/error detection, working memory, and response inhibition (Dajani \& Uddin, 2015). From this perspective, cognitive flexibility has primarily been assessed through behavioral paradigms, such as the Wisconsin Card Sorting Task (WCST) (Grant \& Berg, 1948). Disturbances in cognitive flexibility, as assessed through behavioral tasks, have frequently been associated with psychiatric illnesses, including depressive disorders (Austin et al., 2001; Ceaser et al., 2008; Chamberlain et al., 2006; Trivedi \& Grier, 2014). However, the factors which may contribute to impaired or reduced cognitive flexibility are not fully known.

Monoamine, especially dopamine, activity within prefrontal, striatal, and parietal neural circuits has been shown to play a critical role in regulating cognitive flexibility (Kehagia, Murray, \& Robbins, 2010; Klanker, Feenstra, \& Denys, 2013). As such, most current research has focused on gene variants, or single nucleotide polymorphisms (SNPs), affecting dopamine signaling and how these might contribute to individual differences in cognitive flexibility (Joober, 2002; Logue \& Gould, 2014; Malhotra et al., 2002; Mattay et al., 2003). As an example, the Val158Met polymorphism of catechol-O-methyltransferase (COMT) gene, which has been associated with altered degradation of dopamine (and norepinephrine) primarily in the prefrontal cortex, has been related to differences in performance on the WSCT (Barnett et al., 2007; Malhotra et al., 2002). 
Brain Derived Neurotrophic Factor (BDNF) plays an important role in the growth and differentiation of neurons during brain development, synaptic plasticity, and maintenance of neurons in adult life (Lewin \& Barde, 1996), and has been implicated as a key neurobiological mediator of learning and memory processes, including long-term potentiation (Cunha et al., 2010; Egan et al., 2003; Kovalchuck et al., 2002). Although relatively few studies have examined the significance of BDNF to cognitive flexibility in humans, research in animals has demonstrated that manipulation of central BDNF can influence performance on a rodent analog of the WCST (D'Amore, Tracey, \& Parikh, 2013; Sakata et al., 2013; Savitz et al., 2006). For instance, intra-striatal infusions of BDNF in mice facilitated strategy shifting by minimizing perseverative responding to a previously acquired strategy (D'Amore et al, 2013), and attenuation of activity-dependent BDNF expression in rate impaired spatial memory reversal and contextual memory extinction (Sakata et al., 2013). As such, it is possible that genes regulating the expression of BDNF might also contribute to individual differences in cognitive flexibility.

A common polymorphism in the BDNF gene (Val66Met) produces a Val to Met amino acid substitution of the pro-BDNF sequence, which leads to disruptions of BDNF functioning (Egan et al., 2003). The Met allele has been related to reduced hippocampal and prefrontal volume (Pezawas et al., 2004), and disturbances in learning and memory processes (Bath \& Lee, 2006). However, the potential contribution of this BDNF polymorphism to cognitive control functions, such as cognitive flexibility, has not been extensively explored. Thus, the initial purpose of the present investigation was to examine whether the BDNF Val66Met polymorphism was related to individual differences in cognitive flexibility, as assessed by the WCST.

The BDNF polymorphism has been shown to interact with previously experienced life events in producing several cognitive, affective, and neurobiological outcomes (Caldwell et al., 
2013; Carballedo et al., 2013; Gatt et al., 2009; Perroud et al., 2008). Specifically, among individuals who carried the Met allele of the BDNF gene, early life stressors were associated with deficits in working memory as well as anxiety and depressive symptoms (Gatt et al., 2009). Furthermore, greater frequency of early life stressors was associated with reduced volume in prefrontal and hippocampal regions among Met allele carriers, but not those who were homozygous of the $\mathrm{Val}$ allele (Gatt et al., 2009). Given these findings, it possible that the relationship between previously experienced life stressors and cognitive flexibility might also be moderated by the BDNF Val66Met polymorphism. However, in the context of cognitive flexibility, these relationships might further depend on the nature, or severity, of the stressful experience. In this regard, less severe experiences, such as frequent changes in residence or parent's job/work status, were aligned with enhanced cognitive flexibility (i.e., set-shifting performance on the WCST) later in life (Mittal et al., 2015), whereas experiences of childhood trauma (e.g., physical abuse and neglect) were associated with reduced performance of the WCST among adolescents (Spann et al., 2012). Considering these findings, it was also interest to determine whether the moderating role of the BDNF polymorphism in the relation between traumatic life events and cognitive flexibility was dependent on the nature of the trauma, including general traumas (e.g., serious injury or illness, death of a loved one, separation or divorce of parents), physical punishment, emotional abuse, and sexual abuse.

Additionally, it has previously been reported that males and females differ in their performance of the WCST (Aly et al., 2015; Boone et al., 1993). As well, sex differences have been suggested to contribute to differential sensitivity (e.g., increased risk of depressive and anxiety disorders) to different forms of stressful and traumatic life events (Freedman, et al., 2002; Kendler, Thornton, \& Prescott, 2001; Rudolph \& Hammen, 1999). Thus, as a final aim of 
the present study, we examine whether the moderating role of the BDNF polymorphism in the relationship between different forms of traumatic events and cognitive flexibility was further dependent on sex differences.

\section{METHODS}

\section{Participants}

The present study involved 239 (female: $n=147$ ) Carleton University undergraduate students. Given that BDNF polymorphism frequency varies across cultural groups (e.g., EuroCaucasian vs Asian), all participants were of Euro-Caucasian decent, ranging in age from 17 to $31(M=19.38, S D=3.37)$. None of the participants reported a neurological disorder or learning disability; 24 participants were currently taking anti-depressant and/or anti-anxiety medication, and 2 individuals were using the psychostimulant methylphenidate.

\section{Procedure}

Once signed informed consent was obtained, participants provided a saliva sample for genotyping after which they completed a computerized version of the WCST. Participants then responded to a series of demographic questions as well as a measure of early life trauma, depressive mood, and anxiety. All procedures were approved by the Carleton University Ethics Committee for Psychological Research.

\section{Genotyping}

Samples for genotyping were collected using Norgen collection kits (Norgen Biotek Corp., Thorold, Ontario Canada). Genomic DNA was extracted from the sample collection kits according to the manufacturer's instructions, and diluted to approximately equal concentration (20 ng/ $\mu \mathrm{L}$ ). Samples were sent for genotyping to McGill University and Génome Québec 
Innovation Centre (Montreal, Canada). Using polymerase chain reaction (PCR), the DNA was amplified, and QIAXcel was used to determine amplification status. Shrimp alkaline phosphatase was used to remove all unincorporated deoxyribose nucleoside triphosphates (dNTPs). One probe per marker was used to do a single base extension and the product was desalted using $6 \mathrm{mg}$ of resin. The product was spotted on Agena BioScience 96-well chips using a Samsung Nanodispenser, and the chip read by a Mass Spectrometer. A manual analysis was done for each marker. Primer sequences were as follows:

BDNF Val ${ }^{66}$ Met forward: ACGTTGGATGTACTGAGCATCACCCTGGA

BDNF Val ${ }^{66}$ Met reverse: ACGTTGGATGGCTTGACATCATTGGCTGAC

BDNF Val ${ }^{66}$ Met probe: TCCAACAGCTCTTCTATCA.

The allele distribution for the BDNF Val66Met polymorphism was $157 \mathrm{Val} / \mathrm{Val}, 61$ $\mathrm{Val} / \mathrm{Met}$, and $4 \mathrm{Met} / \mathrm{Met}$, which met Hardy-Weinberg Equilibrium expectations, $\chi^{2}(1)=.484, p=$ 0.486. Due to the relative infrequency of Met homozygotes, we collapsed across $\mathrm{Val} / \mathrm{Met}$ and Met/Met carriers, as done in previous studies (Caldwell et al., 2013). A total of 17 individuals were excluded from the genotype analyses because we were either unable to determine a genotype from the samples provided or the individual chose not to provide a saliva sample.

\section{Cognitive Flexibility: Attentional Set-Shifting}

Cognitive flexibility, assessed through attentional set-shifting, was determined using a computerized version of the WCST, specifically Berg's Card Sorting Task (BCST), provided by the Psychology Experiment Building Language (PEBL) version 0.14 (Mueller \& Piper, 2014). The BCST consists of a 128 card deck with each card containing a different combination of one 
of four shapes, colors, and quantities. Four key cards are displayed at the top of the screen as a guide to help determine to which of the four stacks the deck's up-card is sorted. The deck is revealed one card at a time, and the visible card is matched to key cards depending on the particular rule (unknown to the participant) for a given set. After ten cards have been successfully matched (i.e., the participant has acquired the rule for the first attentional set), the set is completed and the sorting rule changes (also unknown to the participant). The new rule must be discovered using trial and error and through feedback received after each card is sorted. After a card is sorted, the participant is provided with feedback regarding whether it was sorted correctly (i.e., according to the current rule). This process continues until the participant either sorts all 128 cards, or until the participant successfully completes 9 sets/categories (for more information see Fox et al., 2013). The BCST takes approximately 10 minutes to complete.

The primary measures of the BCST are the type of errors the individual makes.

Perseverative errors occur when the individual continues to sort cards according to a previously, but no longer, relevant or correct sorting rule. These types of errors were the central measure of reduced cognitive flexibility within the attentional set-shifting paradigm. In the present study, perseverative errors were computed according to procedures described by Heaton et al. (1993). Non-perseverative errors, by contrast, refer to all other errors. Among the non-perseverative errors are failures to maintain set, which refers to selecting an incorrect card once a sorting rule has been learned (i.e., switching after the fifth correctly sorted card). Failures to maintain a set are thought to represent the individual's distractibility or difficulties maintaining information in working memory (Barcelo \& Knight, 2002). Finally, the BCST also assesses trials to first category, which refers to the speed of which an individual acquires an attentional set. 
Measures

Traumatic life events. The frequency of traumatic life events was assessed using a modified version of the short form of the Early Trauma Inventory - Short Report (ETI-SR; Bremner, Bolus, \& Mayer, 2007). The short form of the ETI-SR is a 27-item self-report questionnaire assessing various types of early life events, including i) general traumas (e.g., serious personal injuries or illness, death or serious illness of a parent, sibling, or friend, family history of violence mental illness, or alcohol or drug abuse), ii) physical punishment, iii) emotional abuse, and iv) sexual events. For each item, participants are asked to indicate the frequency, from 0 (never) to 5 (more than 10 times), of which each event has occurred. Scores for each subscale (i.e., general trauma, physical punishment, emotional abuse, and sexual events) are computed by summing across all items comprising the specific form of life event. The original ETI-S asks respondents to indicate the frequency of specific life events that occurred only prior to the age of 18 . We modified these criteria by asking respondents to indicate the frequency of each life event that occurred between the ages of 0 to 5,6 to 12,13 to 18 , and $18+$. For the present study, since we were specifically interested in examining the relationship the type of trauma (and not the timing of these events) and cognitive flexibility, we summed across all age ranges for each type of trauma. Thus, in the present investigation, we had total frequency scores for general traumas, physical punishment, emotional abuse, and sexual abuse.

Anxiety. State anxiety was assessed using the Spielberger State Trait Anxiety Inventory (STAI) (Spielberger et al., 1983). The 20-item state anxiety scale was used to measure current feelings of anxiety following the TSST. Items ranged from 1 (not at all) to 4 (very much), where higher scores indicate greater state anxiety. Total scores were obtained by summing across all items $(\alpha=.95)$. 
Affective state. The 20-item Positive and Negative Affect Schedule (PANAS; Watson, Clark, \& Tellegen, 1988) was used to assess positive and negative affect post stressor. Responses ranged on a six-point scale from 1 (very slightly or not at all) to 5 (extremely). Positive and negative affect scores were computed by summing across all 10 relevant items for each subscale. Cronbach's alphas were: positive (.85) and negative (.90).

Depression. The 21-item Beck Depression Inventory (BDI) was used as a measure of depressive symptomatology (Beck, Ward, \& Mendelson, 1961). Each item comprises one of four options, ranging from low to high depressive symptomatology. Total scores were calculated by summing across all items $(\alpha=.88)$.

Statistical Analyses

Statistical analyses were performed using SPSS for Windows 18.0 (SPSS Science, Chicago, IL). Univariate analyses of variance (ANOVAs) were used to examine differences in WCST performance indices (i.e., perseverative and non-perseverative errors, failures to maintain an attentional set, and number of trials to learn the first sorting category) as a function of gender and BDNF genotype. Correlational analyses were conducted using Pearson's product moment correlations. Hierarchical linear regression analyses were used to examine the moderating role of gender and BDNF genotype in the relation between different types of trauma and WCST performance indices. Significant moderations were followed up using a web utility for simple slopes (Preacher et al., 2006).

\section{RESULTS}

Performance on the WCST, including the frequency of perseverative and nonpreservative errors, failures to maintain an attentional set, and number of trials required to learn the first category, was not related state anxiety, positive and negative affect, or depressive 
symptoms $(p$ 's $>.05)$. Furthermore, individuals who were currently taking psycho-active medications did not differ in any of the WCST performance indices. As such, in the present study, the observed findings appeared be independent of current mood state or medication status.

An independent samples t-test revealed that males $(M=12.22, S D=5.22)$ committed more perseverative errors than females $(M=10.92, S D=4.60), t(230)=1.99, p<.05$. Males $(M$ $=14.87, S D=5.75)$ also took slightly more trials to learn the first sorting category than females $(M=13.50, S D=4.27), t(150.94)=1.94, p=.05$. In contrast, no sex differences were observed for the frequency of non-perseverative errors or failures to maintain an attentional set ( $p$ 's $>.05)$. Furthermore, as shown in Figure 1, males reported a greater frequency of physical punishment than females, $t(162.91)=4.06, p<.001$. No sex differences were observed for the frequency of general traumas, emotional abuse, or sexual abuse ( $p$ 's $>.05)$.

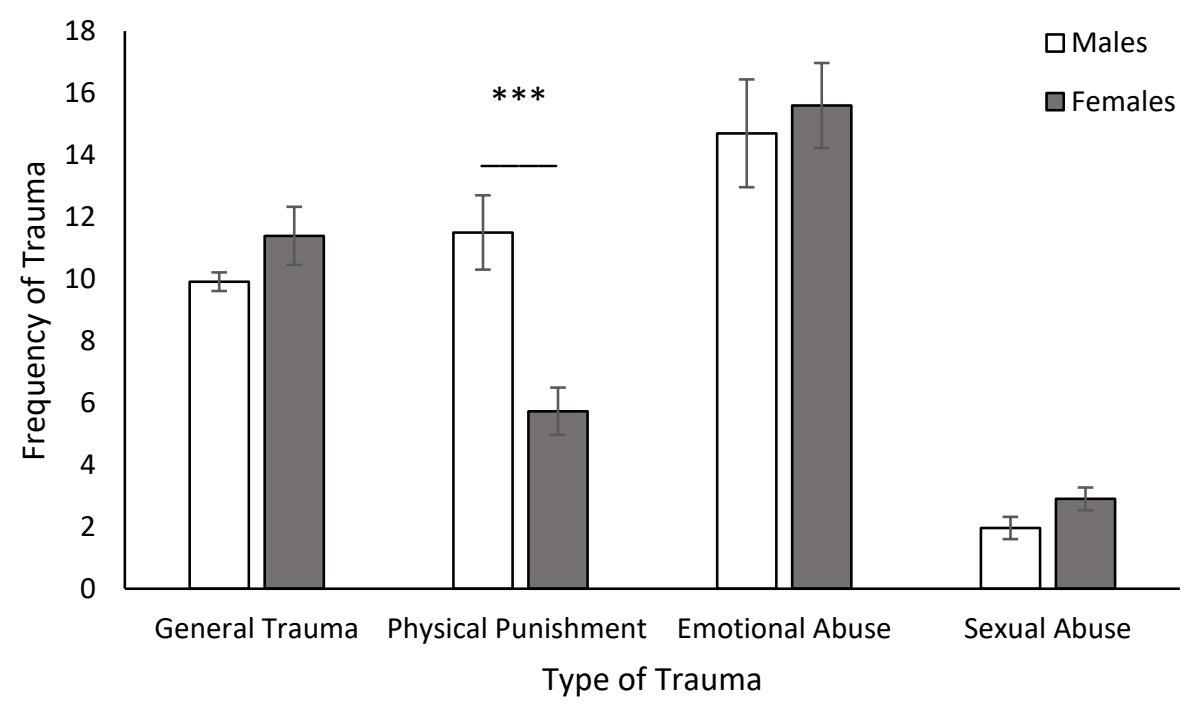

Figure 1. Mean ( \pm S.E.M.) sex differences in the frequency of different forms of traumatic events. 
The BDNF Val66Met polymorphism in relation cognitive flexibility

A univariate ANOVA indicated that individuals who carried the Met allele of the BDNF polymorphism committed significantly more perseverative errors on the WCST (i.e., reduced cognitive flexibility or attentional set-shifting performance) than those who were homozygous for the $\mathrm{Val}$ allele, $F(1,228)=5.57, p<.05$. As well, males committed more perseverative errors than females, $F(1,228)=4.82, p<.05$. No significant Genotype $\mathrm{x}$ Sex interaction, $F(1,228)=$ $1.42, p>.05$, was observed for the frequency of perseverative errors on the WCST. Moreover, no significant BDNF genotype, Sex, or Genotype x Sex differences were observed for the frequency of non-perseverative errors, failures to maintain an attentional set, or number of trials required to learn the first sorting category ( $(p$ 's $>.05)$.

Traumatic life events in relation to cognitive flexibility: moderating role sex and the BDNF Val66Met polymorphism

Zero-order correlations indicated that more frequent perseverative errors (i.e., reduced attentional set-shifting performance) were modestly related to a greater frequency of selfreported general traumas, $r=.14, p<.05$, and physical punishment, $r=.13, p=.057$, but not emotional, $r=.10, p>.05$, and sexual, $r=.08, p>.05$, abuse. Moreover, more frequent nonperseverative errors were related to greater frequency of general traumas, $r=.17, p<.01$, but not other forms of trauma ( $p$ 's $>.05$ ). As well, failures to maintain an attentional set and the number of trials required to learn the first sorting category were unrelated to any type of trauma.

A hierarchical regression analysis revealed a significant General Traumas x Genotype, $\Delta R^{2}=.02, F(1,226)=5.75, p<.05$, General Traumas x Sex, $\Delta R^{2}=.02, F(1,226)=5.69, p<.05$, and a General Traumas x Genotype $\mathrm{x} \operatorname{Sex}, \Delta R^{2}=.05, F(1,226)=6.11, p<.01$, interaction in 
relation to the frequency of perseverative errors on the WCST. As illustrated in Figure 2A, among males, greater frequency of general traumas was modestly, but not significantly, associated with more frequent perseverative errors among Val homozygotes, $b=.11, t=1.83, p$ $=.07$, and this relationship was much stronger those carrying a Met allele, $b=.26, t=4.09, p<$ .001. In contrast, among females, greater frequency of general traumas was not related to perseverative errors among $\mathrm{Val}$ homozygotes, $b=-.04, t=-.95, p>.05$, and only modestly related among females carrying the Met allele, $b=.10, t=2.08, p<.05$ (Figure 2B).
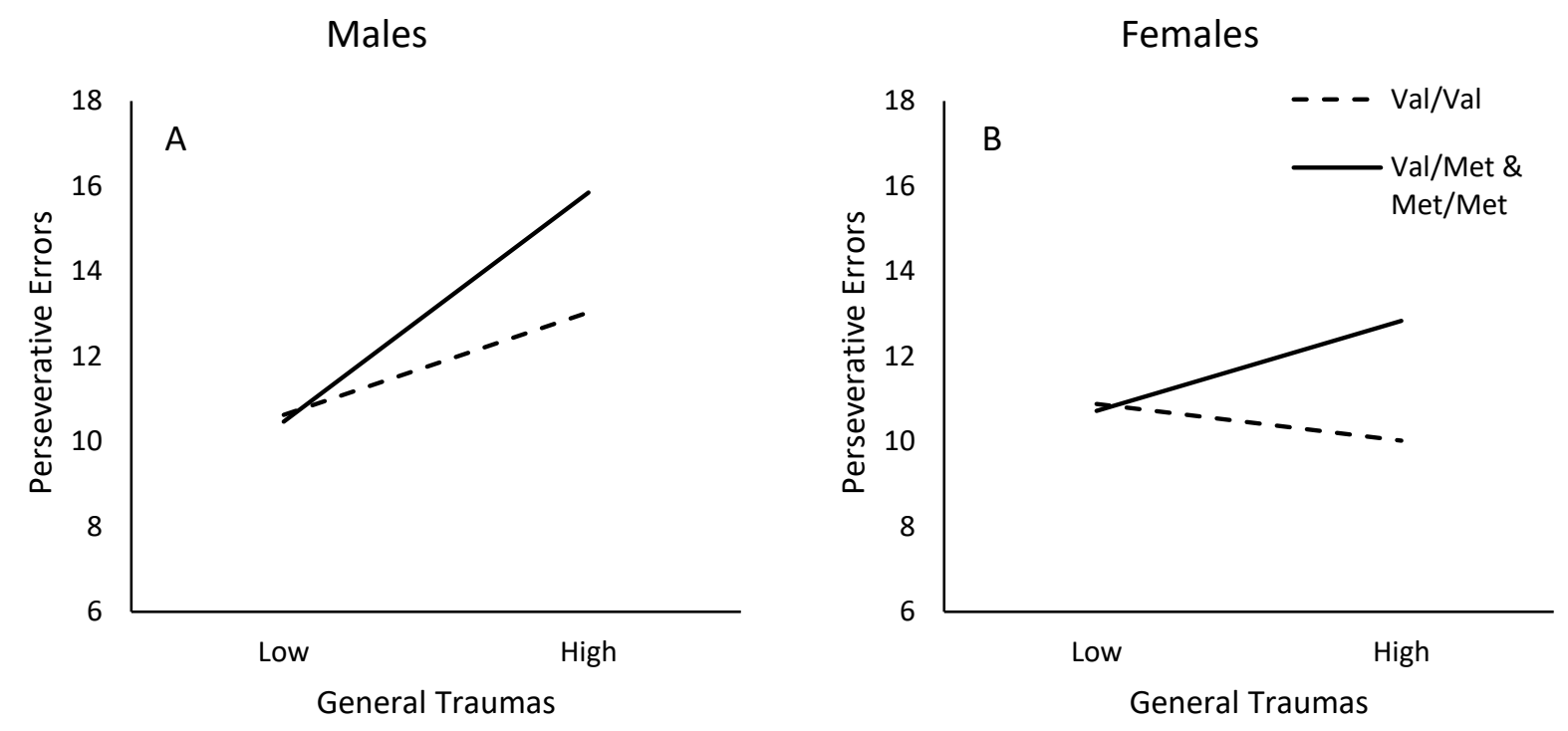

Figure 2. The moderating role of the BDNF Val66Met polymorphism and sex in the relationship between frequency of general traumas and frequency of perseverative errors on the WCST. *Note: low frequency of general traumas $=1$ S.D. below the mean, high frequency of general traumas $=1$ S.D. above the mean 
Similar to the findings observed for general traumas, a Physical Punishment x Genotype $\mathrm{x}$ Sex interaction was also observed in relation to the frequency of perseverative errors, although this interaction was just shy of the .05 statistical significance level, $\Delta R^{2}=.02, F(1,226)=2.85, p$ $=.06$. As illustrated in Figure 3A, among males, greater frequency of physical punishment was related to more frequent perseverative errors among Met carriers, $b=.14, t=2.54, p=.01$, but not among $\mathrm{Val}$ homozygotes, $b=.07, t=1.33, p>.05$. In contrast, among females, physical punishment was not related to the frequency of perseverative errors among Met carriers, $b=.02$, $t=.29, p>.05$, or those who were homozygous for the $V a l$ allele, $b=-.05, t=-1.06, p>.05$ (Figure 3B).
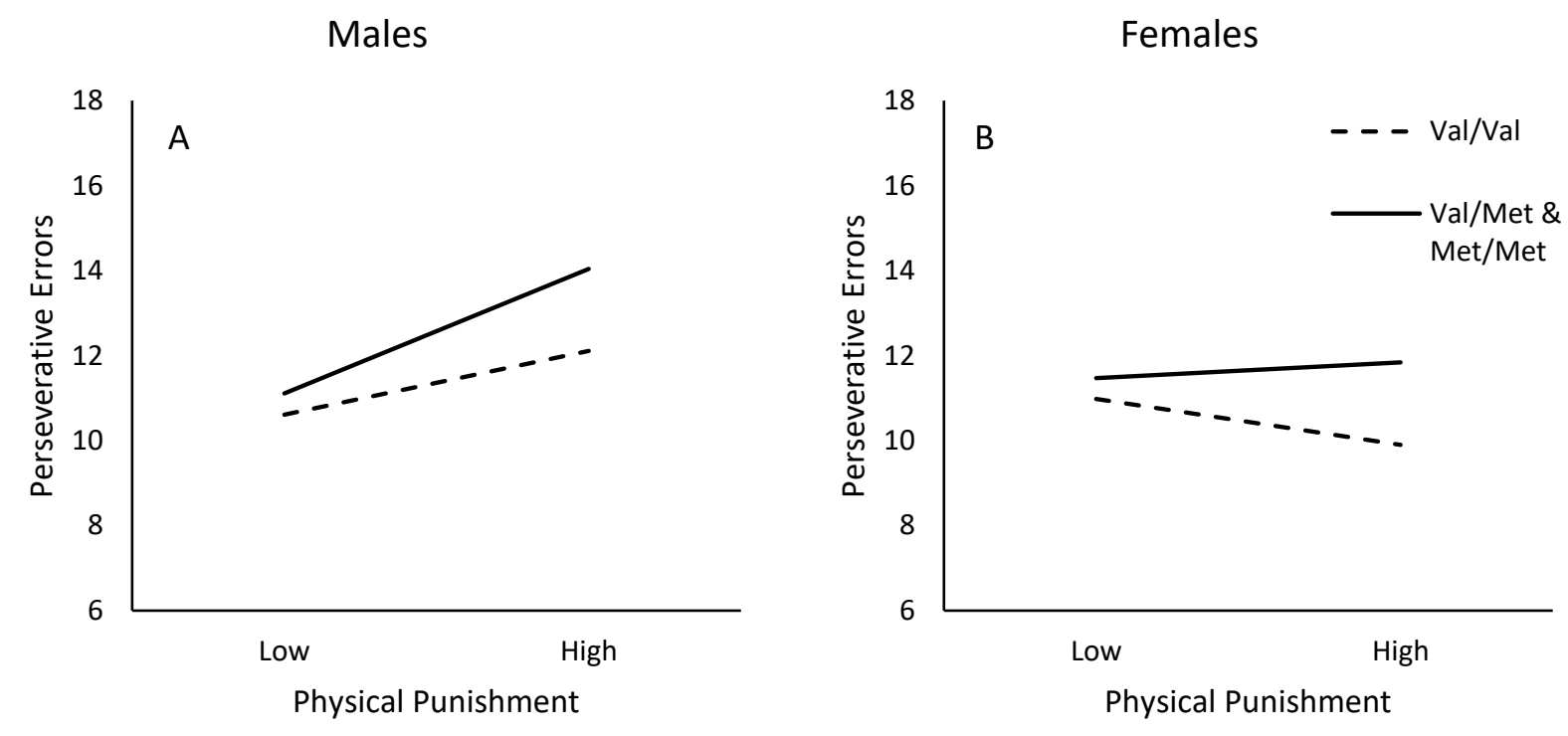

Figure 3. The moderating role of the BDNF Val66Met polymorphism and sex in the relationship between frequency of physical punishment and frequency of perseverative errors on the WCST. *Note: low frequency of physical punishment $=1$ S.D. below the mean, high frequency of physical punishment $=1$ S.D. above the mean 
In contrast to these relations, we did not observe a significant Emotional Abuse $\mathrm{x}$ Genotype $\mathrm{x}$ Sex interaction in relation to the frequency of perseverative errors committed on the WCST, $\Delta R^{2}=.02, F(1,226)=1.99, p=.14$. However, given a prior hypothesis, we proceeded with follow-up simple slopes analysis to determine whether the relation between emotional abuse and perseverative errors varied as a function of BDNF genotype, gender, or a combination of both these variables. In contrast to the relations observed for general trauma and physical punishment, as shown in Figure 4A, among males, emotional abuse frequency was unrelated to perseverative errors for both Met carries, $b=.05, t=1.25, p>.05$, or Val allele homozygotes, $b$ $=-.01, t=-.43, p>.05$. However, among females, greater frequency of emotional abuse was related to more frequent perseverative errors among Met allele carries, $b=.09, t=2.51, p=.01$, but not $V a l$ homozygotes, $b=.02, t=.75, p>.05$ (Figure 4B). 
Males

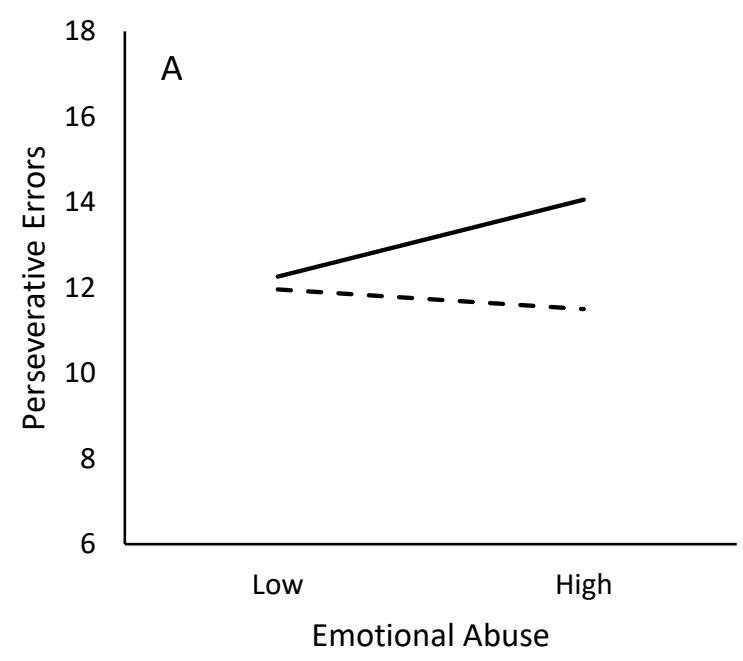

Females

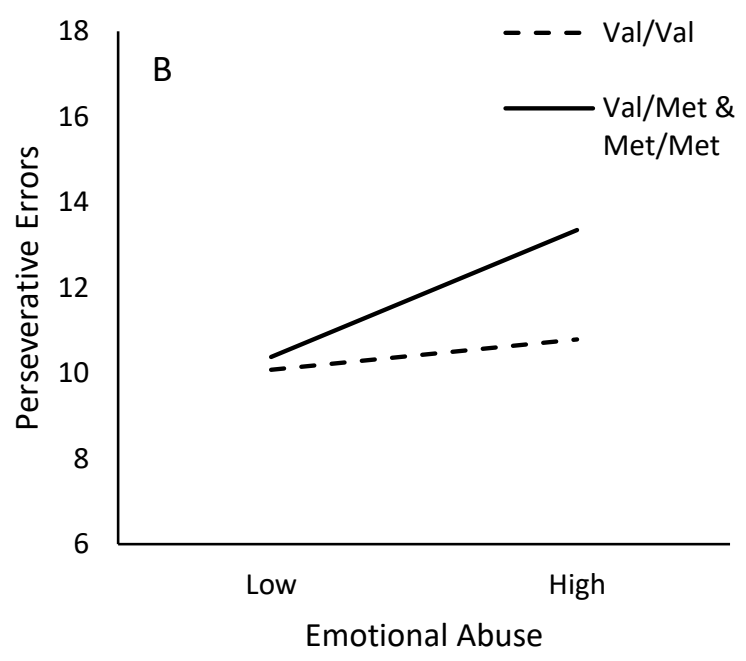

Figure 4. The moderating role of the BDNF Val66Met polymorphism and sex in the relationship between frequency of emotional abuse and frequency of perseverative errors on the WCST. *Note: low frequency of emotional abuse = 1 S.D. below the mean, high frequency of emotional abuse $=1$ S.D. above the mean

Finally, we did not observe a Sexual Abuse x Genotype x Sex interaction in relation to the frequency of perseverative errors, $\Delta R^{2}=.00, F(1,226)=.03, p>.05$. Moreover, as indicated by additional hierarchical regression analyses, the Trauma $\mathrm{x}$ BDNF genotype $\mathrm{x}$ Sex interaction effects were unique to attentional set-shifting performance (i.e., frequency of perseverative errors) as the combination of these variable were not related to non-perseverative errors, failures to maintain an attentional set, or the number of trial required to learn the first sorting category. 


\section{DISCUSSION}

The Met allele of the BDNF Val66Met polymorphism (RS6265) contributes to altered cellular packaging and tracking of BDNF, and has been associated decreased cortical volume in prefrontal and hippocampal regions, impaired learning and memory processes, and increased risk for depressive disorders (Bath \& Lee, 2006; Gatt et al., 2009; Pezawas et al., 2004). In the present investigation, we examined whether this BDNF polymorphism was associated with impaired cognitive flexibility (assessed by the WCST), an ability which has been shown to be impaired among depressed individuals (Trivedi \& Greer, 2014), and the additional contribution of previously experienced traumatic events and sex in this regard.

In the present investigation, in comparison to those who were $\mathrm{Val}$ allele homozygotes for the BDNF gene, individuals who carried the Met allele exhibited reduced attentional set-shifting (or cognitive flexibility), as reflected by more frequent perseverative errors on the WCST. This effect was unique to set-shifting, as the BDNF polymorphism did not contribute to differences in the frequency of non-perseverative errors, including the ability to acquire (i.e., number of trial required to learn the first sorting category) or maintain an attentional set. Moreover, these findings were likely not attributable to mood state, as state anxiety, positive or negative affect, or depressive symptoms were not related to set-shifting ability.

The present findings a similar to those observed among individuals with bipolar disorder, whereby those who carried the Met allele performed significantly more perseverative errors on the WCST than those who were Val homozygotes, but did not differ in the frequency of nonperseverative errors or the number of trials needed to learn the first sorting rule (Rybakowski et al., 2006). As well, these findings are reminiscent of those observed in rodents, wherein selective disruption of activity-dependent BDNF protein expression (i.e., in BDNF mutant rats) did not 
affect working, spatial or fear memory, but contributed to pronounced perseverative responding, reflected by impairments of reversal learning in a spatial memory task as well as in extinction of fear memory (Sakata et al., 2013). Thus, the Met allele of the BDNF gene (or disruptions in BDNF functioning) does not appear to influence the ability to acquire or maintain an attentional set (or behavioral strategy). Instead, it appears to specifically disrupt cognitive flexibility, specifically the ability to shift attention away from a previously, but no longer, appropriate behavioral strategy and towards one that is newly effective.

In addition to BDNF genotype, we observed that sex also contributed to differences in in WCST performance. Specifically, males committed modestly more perseverative errors (reduced set-shifting) than females, but did not differ in the frequency of non-perseverative errors, including the ability to acquire and maintain an attentional set. Consistent with these findings, it has previously been reported, among healthy individuals, women generally performed better on the WCST than men (Aly et al., 2015; Boone et al., 1993), which has led to the suggestion that performance on this behavioral task should be interpreted within the context of sex differences (Boone et al., 1993). The present findings are also in line with the evidence indicating that, through differences in brain structure, function, and chemistry (Cosgrave, Mazure, \& Staley, 2007), males and females differ in certain cognitive abilities (Jorm et al., 2004; Mozley et al., 2001; Weis et al., 2003). Thus, considering sex differences when examining the factors which may contribute to variability in cognitive functioning may be valuable.

As discussed earlier, the BDNF Val66Met polymorphism has previously been shown to moderate the relationship between early life stressors and cognitive, affective, and neurobiological outcomes (Gatt et al., 2009). In essence, among individuals with a greater frequency of early life stressors, those who carried the Met allele of the BDNF gene exhibited 
reduced volume in the prefrontal cortex and hippocampus as well as impaired working memory and elevated symptoms of depression and anxiety (Gatt et al., 2009). Consistent with these findings, in the present investigation, this BDNF polymorphism moderated the relationship between previously experienced traumatic events and cognitive flexibility. Moreover, we observed that the moderating effect of the BDNF polymorphism was specific to the type of trauma experienced relative to sex differences. Specifically, greater frequency of general traumas (e.g., personal injury or illness, death of a loved one, and family disturbances) was associated with reduced cognitive flexibility (as reflected through more frequent perseverative errors on the WCST) among individuals who carried the Met allele, but not among those who were homozygous for the $\mathrm{Val}$ allele. Moreover, although the strength of these relations slightly differed between males and females, greater frequency of general traumas was equally associated with reduced cognitive flexibility in both males and females who carried the Met allele.

The BDNF polymorphism moderated the relationship between physical punishment and cognitive flexibility as well as emotional abuse and cognitive flexibility, but these effects were further dependent on sex. Greater incidence of physical punishment was related to more frequent perseverative errors on the WCST among males who carried the Met allele, but not among females who exhibited this genotype. In contrast, more frequent emotional abuse was associated with decreased cognitive flexibility among female Met carriers, but not male Met carriers. Furthermore, for both males and females, physical punishment and emotional abuse was unrelated to cognitive flexibility among those who were $\mathrm{Val}$ allele homozygotes.

Together, these findings are consistent with evidence indicating that the Met allele of the BDNF gene is generally accompanied by reduced cognitive functioning and increased affective disturbances (Gatt et al., 2009), and that individuals who carry the Met allele are more adversely 
affected stressful and traumatic experiences (Elzinga et al., 2011; Gatt et al., 2009; Hosang et al., 2014). However, to our knowledge, this appears to be the first study to suggest that the relationship between traumatic life events and reduced cognitive flexibility among Met carriers might be dependent on not only the nature of the trauma experienced, but also the contribution gender differences in this regard. That being said, it is not entirely clear as to why, among Met carrier, physical punishment was associated with reduced cognitive flexibility among males, whereas emotional abuse was more related to impaired flexibility among females. However, as previously discussed, it has been reported that males and females not only differ in the occurrence of specific types of stressful and traumatic events, by also in their sensitivity and responses to different stressors (Freedman, et al., 2002; Kindler, Thornton, \& Prescott, 2001; Tolin \& Foa, 2006). From this perspective, the detrimental effects associated with the Met allele of the BDNF gene on cognitive and affective functioning might vary between males and females depending on the nature of the traumatic events experienced.

Several limitations should be considered regarding the findings of the present study. The assessment of early life trauma (i.e., the ETI-SR) was determined through a self-report measure, which might have been susceptible to memory biases and difficulties recalling early life events. Moreover, given that the frequency of gene polymorphisms varies across ethnic groups, and that their influence on behavior might vary by ethnicity, the present sample included only EuroCaucasian individuals. Therefore, the present findings might not be generalizable to other ethnicities. As well, in the present investigation, symptoms of depression were not associated with set-shifting performance. This was not entirely surprising given that disturbed set-shifting was particularly evident among severely depressive or elderly individuals (Austin et al., 2001; Grant et al., 2001), and the present sample comprised young and high functioning individuals, 
with relative low levels of depression. This said, it is possible that the contribution of disturbed set-shifting to depressive pathology might be most apparent under conditions where a challenge was experienced (e.g., following an acute or chronic stressor).

Taken together, the present findings indicate that early life stressors and the BDNF polymorphism might be synergistically related to disturbed attentional set-shifting. In essence, among Met allele carriers of the BDNF gene, a history of early life stressors might not impair the initial acquisition of information or the adoption of a particular behavioral strategy. However, as the contingencies within a given situation change, the presence of the Met allele, for whatever reason, might be accompanied by difficulties disengaging from a previously established strategy and acquiring a new, effective behavioral response. These findings might provide further insight related to the factors that might contribute to depressive disorders, which have been associated disturbances of cognitive flexibility. 


\title{
CHAPTER 3
}

\section{Cognitive flexibility in the context of stress and depressive disorders: The Cognitive Flexibility Questionnaire}

\begin{abstract}
The present investigation examined whether aspects of cognitive flexibility relevant to stressful experiences were related to low-grade depressive symptoms. Study 1 presents the cognitive flexibility questionnaire (CFQ), a brief self-report measure that incorporated cognitive flexibility as reflected through attentional control, stressor appraisals, and coping. The CFQ largely comprised two factors, cognitive control and cognitive resources and had adequate construct validity and reliability. Low levels of cognitive flexibility remained significantly associated with heightened depressive symptoms, even after accounting for factors such as emotion regulation and rumination. In Study 2, cognitive flexibility moderated the relation between dysfunctional attitudes and negative cognitive style on symptoms of depression. Study 3 revealed that the relation between reduced cognitive flexibility and elevated depressive symptoms was mediated by negative stressor appraisals and the endorsement of ineffective coping. Together, the present findings provide insight into the processes through which disturbances in cognitive flexibility might promote depressive symptoms.
\end{abstract}




\section{INTRODUCTION}

Depressive disorders have frequently been associated with cognitive dysfunction, particularly disturbed cognitive flexibility (Trivedi \& Greer, 2014), which appears to be especially marked when processing negative emotional information (Deveney \& Deldin, 2006). Disturbed cognitive flexibility might not only facilitate repetitive negative thinking but could potentially contribute to the maintenance of negative information processing biases, such as those related to attention and memory (Gotlib \& Joormann, 2010; Mathews \& MacLeod, 2005). In fact, the inability to disengage from negative emotional information may be a central characteristic of depression (Joorman, Talbot, \& Gotlib, 2007).

Cognitive flexibility has predominantly been assessed through laboratory-based behavioral tasks, such as the Wisconsin Card Sorting Test (WCST; Grant \& Berg, 1948) and the intra- and extra-dimensional (ID/ED) shift task (Downes et al., 1989). Although useful in identifying the presence of disturbances in cognitive functioning among depressed individuals, performance on these measures does not capture the processes by which disturbed cognitive flexibility can be manifested in the context of stressful events, which are germane to the emergence and maintenance of depressive pathology (Kendler, Karkowski, \& Prescott, 1999).

\section{Cognitive Flexibility in the Context of Stressful Events and Depressive Disorders}

Cognitive flexibility refers to the ability to modify "cognitive sets" in response to changes in the environment or the individual's intentions. At the most basic level, this ability involves shifting attention between multiple sources of information. During stressful situations, this might entail directing attention towards information relevant to resolving a stressor, and away from nonessential information. As the characteristics of the stressor change, this might further involve shifting attention to newly relevant information. Given the limited capacity of 
cognitive resources (e.g., working memory) (Baddeley, 2003), the ability to focus on relevant information might be highly functional in dealing with stressful situations effectively.

Stressful events often provoke negative emotional responses, which can bias cognitive processing (e.g., attention and memory) towards negative information (Mathews \& MacLeod, 2005; Yiend, 2010). Initially, this may serve to direct necessary resources towards issues that must be dealt with. However, a preoccupation with negative emotional information may ultimately contribute to exacerbated negative mood (Joorman et al., 2007). Indeed, depressed individuals typically exhibit difficulties disengaging from negative emotional information (Deveney \& Deldin, 2006; Joorman et al., 2007). Thus, cognitive flexibility in this context might be manifested through the ability to disengage from negative emotional information, allowing the individual to identify more relevant sources information to cope effectively with a stressful situation.

When individuals encounter a stressful situation, an appraisal is made as to whether the event poses a threat to their well-being and the extent to which they can exert control over the situation, which ultimately gives rise to a specific emotional response (Lazarus \& Folkman, 1984). Depressed individuals tend to make negative appraisals to stressful situations (Abramson, Metalski, \& Alloy, 1989; Folkman \& Lazarus, 1986), although it might be the persistent application of negative appraisals (or attributions) across different stressful situations might serve to maintain negative mood, and ultimately promote depressive pathology (Fresco, Williams, \& Nugent, 2006; Fresco, Rytwinski, \& Craighead, 2007). Cognitive flexibility in this context might be reflected through the ability to modify appraisals according to the characteristics of the situation, and thus buffer against the emergence of depressive symptoms. Furthermore, the appraisal process is dynamic, such that the initial appraisal of a situation is 
typically followed by a reappraisal (Lazarus \& Folkman, 1984). Failure or difficulty in reappraising stressful situations appropriately might contribute to the maintenance of negative emotional responses, and hence the emergence of depressive pathology (Garnefski \& Kraaij, 2006; Gross \& John, 2003). In essence, reappraisals involve shifting "cognitive sets" and thus may be a way through which cognitive flexibility is expressed in stressful situations.

Cognitive flexibility may also play a role in the selection and endorsement of particular coping strategies. For instance, searching for multiple or alternative solutions to a problem and reinterpreting negative events in terms of potentially positive experiences (i.e., cognitive restructuring) requires some degree of cognitive flexibility. In contrast, emotion-focused coping, such as rumination (i.e., brooding), likely reflects cognitive inflexibility encompassing stereotypical and perseverative thinking (Nolen-Hoeksema, Wisco, \& Lyubomirsky, 2008). In addition, cognitive flexibility might underpin coping flexibility, which can take several forms, including having a broad coping repertoire, a well-balanced coping profile, cross-situational variability in the strategy deployed, and perceived ability to cope with varied changes (Cheng, Lau, \& Chan, 2014). Disturbed cognitive flexibility (e.g., an inability to generate multiple alternative coping methods), in contrast, might contribute to the tendency for depressed individuals to rigidly apply the same coping strategies across diverse situations, even if they have proven to be ineffective (Cheng, 2001; Fresco et al., 2006; 2007).

Among the ways in which cognitive flexibility can be manifested in stressful situations, they can generally be clustered into three core processes: 1) attention (e.g., attentional setshifting) 2) appraisals (e.g., changes in appraisals or reappraisal) and 3) coping methods (e.g., generating and switching between multiple coping strategies). Several behavioral and self-report measures have been developed to assess each of these aspects of cognitive flexibility. Yet, there 
are no measures that encompass all of the components of cognitive flexibility within a single questionnaire.

\section{Development of the Cognitive Flexibility Questionnaire (CFQ)}

The Cognitive Flexibility Inventory is a self-report measure that addresses aspects of cognitive flexibility relevant to stressful situations and depressive pathology (Denis \& Vander Wal, 2010). This measure considers the ability to perceive multiple alternative explanations for life occurrences, the generation of multiple alternative solutions to difficult situations (alternatives component), and the tendency to perceive difficult situations as controllable (control component). This instrument was primarily designed to assess the type of cognitive flexibility necessary for individuals to challenge and replace maladaptive thoughts with more balanced and adaptive thinking, and to assess the effectiveness of thought challenging techniques in cognitive behavioral treatments for depression and other psychopathological conditions (Denis \& Vander Wal, 2010).

It was of interest in the present investigation to capture several additional factors that are not the focus of the Cognitive Flexibility Inventory that might be tightly linked to mood disturbances. Difficulties in disengaging from negative cognitive and emotional states might be a defining feature of dysphoric mood (Joorman et al., 2007). Thus, we assessed a questionnaire designed to tap individual differences in cognitive flexibility in the presence of negative cognitive and emotional states, which are often precipitated by stressful events. Indeed, Accordingly, the CFQ was designed to measure cognitive flexibility in the context of stressful events reflected through three core processes: 1) cognitive (attentional) control over negative thoughts and emotions, 2) changes of reappraisals in order to attenuate negative emotional responses, and 3) the capability of generating multiple alternative coping strategies in the 
presence of distracting or potentially irrelevant information (e.g., negative thoughts, memories, or emotions).

The development of the CFQ was influenced by several constructs that were also included in the Cognitive Flexibility Inventory, particularly those concerning appraisals and coping. However, the two scales differ in several important respects. Whereas the Cognitive Flexibility Inventory assesses the type of cognitive flexibility necessary for individuals to challenge and replace maladaptive thoughts (Denis \& Vander Wal, 2010), the CFQ was meant to assess the self-perceived ability to disengage, or shift attention away, from stressor-elicited negative cognitive and emotional states. Fundamentally, whereas the Cognitive Flexibility Inventory assesses the tendency to perceive difficult situations as controllable, the CFQ assesses cognitive control over negative thoughts and emotions that are elicited by stressful situations. In this regard, it was hypothesized that low levels of cognitive control would facilitate repetitive negative thinking (and rumination) as well as contribute to the maintenance of negative, cognitive processing biases (e.g., attention and memory), and hence the maintenance of negative mood. Moreover, the Cognitive Flexibility Inventory assesses the ability to perceive multiple alternative explanations for life occurrences. The CFQ, in contrast, measures the extent to which individuals perceives themselves as capable of modifying their appraisal (i.e., reappraisal) of a stressful situation in order control or change their cognitive and emotional states. As with cognitive control, it was hypothesized that difficulties in reappraisal would serve to maintain negative cognitive and emotional states elicited by a stressful event. Finally, whereas the Cognitive Flexibility Inventory assesses the ability to generate multiple alternative solutions to difficult situations, the CFQ extends this by assessing whether individuals are still capable of generating multiple alternative coping options/solutions despite the presence of negative 
thoughts, memories, and emotions. It was hypothesized that a preoccupation with distracting emotional information may hinder the ability to generate multiple effective coping strategies, thus contributing to the endorsement of a limited number of coping methods.

Study 1 describes the newly-developed brief self-report measure of cognitive flexibility that would be relevant in the context of stressful experiences and depressive disorders, and that captures process that are not readily assessed by other self-report measures. A second study investigated whether cognitive flexibility, as assessed by the CFQ, moderated dysfunctional attitudes and negative cognitive style in predicting symptoms of depression. Finally, a third study examined whether cognitive flexibility was associated with stressor appraisals and coping styles, and how these, in turn, were related to depressive symptoms.

\section{Study 3 A. Development and psychometric properties of the CFQ}

The aim of Study 1 was to develop and refine the factor structure and reliability of a set of items that provisionally comprised the Cognitive Flexibility Questionnaire. To determine the construct validity of the final form of the CFQ, we examined the relation between this measure and the Cognitive Flexibility Inventory (Denis \& Vander Wal, 2010) as well as several measures (described below) that may reflect cognitive flexibility in stressful situations. This study also examined whether the CFQ provided additional or unique value in predicting stressor-related processes (appraisals and coping), above and beyond that of the Cognitive Flexibility Inventory.

Finally, we examined whether the CFQ was predictive of depressive symptoms after controlling for processes, such as perseverative negative thinking and rumination, which have previously been associated with depression. 


\section{METHODS}

Based on a detailed review of the literature pertaining to stress, coping, and emotion regulation as well as executive functions/cognitive control and cognitive biases among depressed individuals, 116 items were generated that reflected cognitive flexibility/inflexibility in the context of stressful experiences. The preliminary items were presented to 25 graduate students in Psychology and Behavioral Neuroscience who responded to the items and provided feedback concerning which of the preliminary items were redundant or worded in a confusing or ambiguous manner. Items were further eliminated based on low inter-item correlations $(r<.20)$, redundancy $(r>.80)$, confusing or ambiguous wording, or respondents' interpretations of the item not fully representing the current conceptualization of cognitive flexibility. Based on these analyses, we retained 44 items which were used in the present study.

\section{Participants and Procedure}

Participants comprised 314 Carleton University undergraduates ( $n=224$ females), with a mean age of $19.82(S D=3.96)$. Self-reported ethnicities included Caucasian $(72.6 \%, n=228)$, Black $(5.7 \%, n=18)$, Asian $(4.5 \%, n=14)$, Arab (4.1\%, $n=13)$, South Asian $(3.8 \%, n=12)$, Hispanic (2.2\%, $n=7)$, Aboriginal $(1.9 \%, n=6)$, South East Asian, 1.0\% $(n=3)$, and other (e.g., mixed ethnicity, $4.1 \%, n=13$ ). Participants were recruited through the university's computerized recruitment system to complete an online survey in which they responded to the 44 item CFQ, which was introduced as follows:

\footnotetext{
"The purpose of this questionnaire is to determine what you generally think/feel/do when stressful situations provoke negative thoughts and emotions. Of course, you may act differently depending on the situation, but try to think of what you usually think/feel/do when you are stressed or upset. Using the scale below, indicate the extent to which agree or disagree with the following statements.

Generally, in stressful situations ... (specific question follows here)"
} 
Participants responded to each statement on a scale ranging from 1 (strongly disagree) to 7 (strongly agree). In addition, participants completed several measures that assessed a similar or the opposite construct, and their current depressive symptoms. Upon completion of the study, participants were debriefed (verbally and in writing) and compensated with course credit.

\section{Measures}

The Cognitive Flexibility Inventory (CFI; Denis \& Vander Wal, 2010), a 20 item measure assessed two aspects of cognitive flexibility: 1) the ability to perceive multiple alternative explanations for life occurrences and to generate multiple alternative solutions to difficult situations (alternatives), 2) and the tendency to perceive difficult situations as controllable (control). Each statement was rated by the participant from 1 (strongly disagree) to 7 (strongly agree). Scores for each subscale were computed by first reversing item scores where relevant, and then summing the appropriate items for each subscale. The internal reliabilities for the alternatives and control subscales were .93 and .85 , respectively.

The Coping Flexibility Questionnaire (COFLEX; Vriezekolk et al, 2012) is a 13-item questionnaire assessing the capability of switching between assimilative and accommodative coping strategies (versatility) and the capability of generating and considering coping options, and appraising the suitability of a coping strategy in a given situation (reflective coping). Each statement was rated from 1 (seldom or never) to 4 (almost always), and higher scores indicated greater coping flexibility. Scores for each subscale were computed by summing all relevant items. The internal reliabilities for the versatility and reflective coping subscales were .78 and .65 , respectively.

The Emotion Regulation Questionnaire (ERQ; Gross \& John, 2003) is a 10-item questionnaire assessing individual differences in the habitual use of two emotion regulation 
strategies: cognitive reappraisal and expressive suppression. All items are rated on a scale from 1 (strongly disagree) to 7 (strongly Agree). Scores were computed by summing all respective items for the cognitive reappraisal and expressive suppression subscales, with higher scores indicating greater use of that particular emotion regulation strategy. The internal reliabilities for the cognitive reappraisal and expressive suppression subscales were .90 and .80 , respectively.

The Ruminative Response Scale (RRS; Treynor, Gonzalez \& Nolen-Hoeksema, 2003) is a widely used 22-item questionnaire assessing ruminative response styles to sad or depressed mood. For each statement, participants respond on a scale from 1 (almost never) to 4 (almost always). The total rumination score was computed by summing all 22 items. The internal reliability of the RRS in the present study was .94 .

The Perseverative Thinking Questionnaire (PTQ; Ehring et al., 2011) is a 15-item questionnaire assessing content-independent repetitive negative thinking. Participants respond to each item on a scale 0 (never) to 4 (almost always) and a total score was computed by summing all 15 items. The internal reliability of the PTQ in the present study was .96.

The Beck Depression Inventory (BDI; Beck, 1961) is a widely used 21-item measure which assesses the intensity of depressive symptoms. For each item, participants respond to one of four options which range from low to high depression symptomatology. Total scores were calculated by summing across all 21 items. The internal reliability of the BDI in the present study was .92 .

\section{RESULTS}

Exploratory Factor Analysis

An initial principal components analysis with Promax rotation (given that the factors were expected to be correlated) was conducted to explore the factor structure of the 44 item CFQ 
and to select the final items for this measure. The Kaiser measure of sampling adequacy was .95 and Bartlett's Test of Sphericity was $\chi^{2}(946)=8275.46, p<.001$. Although inspection of eigenvalues and the scree plot suggested a 7 -factor solution, only the first 2 factors explained greater than $10 \%$ of the total variance across items (Factor $1=39.11 \%$ and Factor $2=11.02 \%$ ). Furthermore, examination of the unrotated loadings suggested that factors 3 to 7 were not major components as they had fewer than 3 items with substantial (> .40) factor loadings (Zwick \& Velicer, 1986). A parallel analysis, in which the actual eigenvalues were compared to average eigenvalues derived from a series of randomly generated data sets (in this case 100 samples), also suggested the presence of 2 factors explaining $50.13 \%$ of the variance.

Based on these analyses, 18 items were eliminated from the initial 44-item pool based on poor factor loadings $(<.40)$ onto the 2 primary factors, those that appeared redundant, or were not consistent with the constructs represented by the two primary factors. Using Promax rotation, a second principal components analysis was conducted on the remaining 26 items, wherein the number of factors extracted was restricted to 2 . The Kaiser measure of sampling adequacy was .94 and Bartlett's Test of Sphericity was $\chi^{2}(325)=4349.79, p<.001$. Table 1 presents the factor structure of the 26-item CFQ as well as the eigenvalues and percentage of variance accounted for by each of the 2 factors. Scores for each factor/subscale were computed by taking means across all appropriate items (indicated in bold in Table 1).

After examining the content represented by the items within each factor, we named factors 1 and 2 Cognitive Control and Cognitive Resources, respectively. The cognitive control factor largely contains items pertaining to attentional and inhibitory control (e.g., "It is easy for me to shift my attention to other things if I am upset") or lack of control (e.g., "It's hard for me to ignore negative emotions once they have been provoked"). The cognitive resources factor, by 
contrast, is composed of items that mostly reflect appraisal, reappraisal (e.g., "I manage my thoughts or feelings by reframing the situation"), and coping processes (e.g., "I take the time to think of several ways to best cope with the situation"). The correlation between the two factors was .59 , suggesting a moderate degree of overlap between the factors.

To determine the plausibility of a single factor measure, a composite cognitive flexibility score was computed by taking the mean across all 26 items. This single factor measure yielded an internal reliability of .94 , suggesting that a composite score can be computed to assess cognitive flexibility in general, or subscale scores can be computed to assess specific dimensions of cognitive flexibility. 
Table 1. Promax Rotated Pattern Matrix for the CFQ.

Item

Factor 1 Factor 2

1. I get easily distracted by upsetting thoughts or feelings.*

$-.19$

2. My thoughts and emotions interfere with my ability to concentrate.*

.84

$-.18$

3. It's hard for me to shift my attention away from negative thoughts or feelings.*

4. I have a hard time managing my emotions.*

5. It's difficult for me to let go of intrusive thoughts or emotions.*

6. It is easy for me to ignore distracting thoughts.

7. I feel like I lose control over my thoughts and emotions.*

8. I find it easy to set-aside unpleasant thoughts or emotions.

9. It's hard for me to put things in perspective when I'm upset.*

10. It's hard for me to ignore negative emotions once they have been provoked.*

11. It is easy for me to shift my attention to other things if I am upset.

12. I can easily suppress upsetting memories.

13. I can remain in control of my thoughts and emotions.

14. It is easy for me to reassess a negative experience into a positive one.

15. Putting a positive spin on a bad experience comes fairly easy to me.

16. I find it easy to look for something positive, even when I am stressed.

17. I take the time to think of more than one way to resolve the problem.

18. I approach the situation from multiple angles. 
20. I take the time to see things from different perspectives before reacting. $\quad-.12$

21. I take the time to think of several ways to best cope with the situation. $\quad .13 \quad \mathbf{. 7 0}$

22. I weigh many options before choosing how to take action.

23. I manage my thoughts or feelings by reframing the situation.

24. I control my thoughts and feelings by putting the situation in context.

25. I can easily think of multiple coping options before deciding how to respond.

26. I control negative thoughts and emotions by modifying the way I think about the situation.

Eigenvalue

$\operatorname{Mean}(S D)$

Cronbach's $\alpha$

Note: * reversed items. Bold indicates the factor in which the items where retained.

\section{Construct, Convergent, and Incremental Validity}

As shown in Table 2, the CFQ composite (total) score as well as both subscale scores were positively related to both components of the Cognitive Flexibility Inventory. Although the correlations were all significant, they were of moderate size, suggesting that the two measures also have distinctive properties. Additional construct validation was obtained by examining the correlation between the CFQ and several measures assessing emotion regulation, coping flexibility, perseverative negative thinking, and rumination. Higher composite scores on the CFQ were associated with greater utilization of cognitive reappraisal, but not expressive suppression (i.e., inhibiting emotional responses). In addition, higher composite scores on the CFQ were also 
associated with greater coping versatility, whereas lower scores on the CFQ were associated with increased perseverative negative thinking and rumination. As indicated by the relations in Table 2 , the subscales comprising the CFQ were predictive of different processes relevant to stressful events and depressive disorders. Specifically, the cognitive control subscale was aligned with perseverative thinking and rumination, whereas the cognitive resources subscale was more strongly associated with coping flexibility. For comparison purposes, Table 2 also presents the correlations between both components of the Cognitive Flexibility Inventory and the validation measures previously outlined (i.e., emotion regulation, coping flexibility, perseverative negative thinking and rumination). Although, the CFQ and the Cognitive Flexibility Inventory were both similarly related to versatility in coping and rumination, several noteworthy differences were apparent between the two measures. Whereas the CFQ was a stronger predictor of cognitive reappraisal and perseverative negative thinking, the Cognitive Flexibility Inventory was more aligned with reflective coping and expressive suppression. 
Table 2. Zero-order correlations comparing the relation between the CFQ and CFI and validation measures of cognitive flexibility/rigidity.

\begin{tabular}{|c|c|c|c|c|c|c|c|c|}
\hline & $\begin{array}{l}\text { Control } \\
(\mathrm{CFI})\end{array}$ & $\begin{array}{c}\text { Alternatives } \\
(\mathrm{CFI})\end{array}$ & $\begin{array}{c}\text { Versatility } \\
\text { (COFLEX) }\end{array}$ & $\begin{array}{c}\text { Reflective } \\
\text { Coping } \\
\text { (COFLEX) }\end{array}$ & $\begin{array}{c}\text { Cognitive } \\
\text { Reappraisal } \\
\text { (ERQ) }\end{array}$ & $\begin{array}{c}\text { Expressive } \\
\text { Suppression } \\
\text { (ERQ) }\end{array}$ & $\begin{array}{c}\text { Rumination } \\
\text { (RRS) }\end{array}$ & $\begin{array}{c}\text { Perseverative } \\
\text { Thinking } \\
\text { (PTQ) }\end{array}$ \\
\hline CFQ total & $.54 * * *$ & $.49 * * *$ & $.54 * * *$ & .11 & $.58 * * *$ & .01 & $-.52 * * *$ & $-.67 * * *$ \\
\hline CFQ Cognitive Control & $.55 * * *$ & $.33 * * *$ & $.48 * * *$ & .02 & $.52 * * *$ & .03 & $-.58 * * *$ & $-.73 * * *$ \\
\hline CFQ Cognitive Resources & $.38 * * *$ & $.66^{* * *}$ & $.52 * * *$ & $.24 * * *$ & $.52 * * *$ & -.03 & $-.27 * * *$ & $-.38 * * *$ \\
\hline CFI Control & - & - & $.49 * * *$ & .08 & $.32 * * *$ & $-.17 * *$ & $-.50 * * *$ & $-.56 * * *$ \\
\hline CFI Alternatives & - & - & $.44 * * *$ & $.37 * * *$ & $.37 * * *$ & $-.12 *$ & $-.20 * * *$ & $-.21 * * *$ \\
\hline
\end{tabular}

Note. CFQ = Cognitive Flexibility Questionnaire; CFI = Cognitive Flexibility Inventory; COFLEX = Coping Flexibility Questionnaire; ERQ = Emotion Regulation Questionnaire; RRS = Ruminative Response Scale; PTQ = Perseverative Thinking Questionnaire $* p<.05, * * * p<.001$

Table 3. Partial correlations between the CFQ and validation measures of cognitive flexibility/rigidity after controlling for the Cognitive Flexibility Inventory.

\begin{tabular}{lcccccc}
\hline & $\begin{array}{c}\text { Versatility } \\
\text { (COFLEX) }\end{array}$ & $\begin{array}{c}\text { Reflective } \\
\text { Coping } \\
\text { (COFLEX) }\end{array}$ & $\begin{array}{c}\text { Cognitive } \\
\text { Reappraisal } \\
\text { (ERQ) }\end{array}$ & $\begin{array}{c}\text { Expressive } \\
\text { Suppression } \\
\text { (ERQ) }\end{array}$ & $\begin{array}{c}\text { Rumination } \\
(\text { RRS) }\end{array}$ & $\begin{array}{c}\text { Perseverative } \\
\text { Thinking } \\
(\text { PTQ) }\end{array}$ \\
\hline CFQ total & $.31 * * *$ & -.06 & $.46 * * *$ & $.16^{* *}$ & $-.37 * * *$ & $-.57 * * *$ \\
CFQ Cognitive Control & $.26 * * *$ & -.08 & $.43 * * *$ & $.17 * *$ & $-.42 * * *$ & $-.62 * * *$ \\
CFQ Cognitive Resources & $.30 * * *$ & .01 & $.38 * * *$ & .09 & $-.13 *$ & $-.28 * * *$
\end{tabular}

Note. CFQ = Cognitive Flexibility Questionnaire; COFLEX = Coping Flexibility Questionnaire; ERQ = Emotion Regulation Questionnaire; RRS = Ruminative Response Scale; PTQ $=$ Perseverative Thinking Questionnaire $* p<.05 * * p<.01 * * * p<.001$ 
As shown in Table 3, when controlling for the Cognitive Flexibility Inventory, the composite CFQ as well as both subscales remained significantly associated with cognitive reappraisal and coping versatility as well as perseverative negative thinking and rumination, suggesting that the CFQ provides unique variance in predicting these processes. However, several noteworthy reductions in correlation strength between the CFQ and the validation measures were observed after controlling for the Cognitive Flexibility Inventory. First, the cognitive resources subscale of the $\mathrm{CFQ}$ was no longer significantly associated with reflective coping, suggesting that the Cognitive Flexibility Inventory accounts for the bulk of the variance in this form of coping. Additionally, a positive correlation between the CFQ and cognitive control subscale and expressive suppression was observed, suggesting a statistical suppression effect likely due to the negative correlation between the expressive suppression and the Cognitive Flexibility Inventory. Thus, while both measures assess cognitive flexibility, the CFQ and the Cognitive Flexibility Inventory are predictive of different processes relevant to stressful events and depressive pathology.

Table 4 shows that lower composite CFQ scores as well as both subscales were directly related to depressive symptoms. Similarly, lower scores on the control $(r=-.46)$ and alternatives $(r=-.24)$ components of the Cognitive Flexibility Inventory were also related to elevated depressive symptoms, although to a lesser extent. As well, the relations between the CFQ and subscales and depressive symptoms remained highly significant even after independently controlling for both components of the Cognitive Flexibility Inventory as well as the Emotion Regulation Questionnaire, Coping Flexibility Questionnaire, Perseverative Thinking Questionnaire, and the Ruminative Response Scale. Thus, the constructs assessed within the 
CFQ reflect features associated with depressive symptoms that are not addressed by other measures. 
Table 4. Partial correlations between the CFQ and depressive symptoms after controlling for other measures assessing aspects of cognitive flexibility/rigidity.

\begin{tabular}{|c|c|c|c|c|c|c|c|c|c|}
\hline & \multicolumn{9}{|c|}{ Depressive symptoms controlling for } \\
\hline & $\begin{array}{l}\text { Zero-order } \\
\text { correlation }\end{array}$ & $\begin{array}{c}\text { Control } \\
\text { (CFI) }\end{array}$ & $\begin{array}{l}\text { Alternatives } \\
(\mathrm{CFI})\end{array}$ & $\begin{array}{c}\text { Versatility } \\
\text { (COFLEX) }\end{array}$ & $\begin{array}{c}\text { Reflective } \\
\text { Coping } \\
\text { (COFLEX) }\end{array}$ & $\begin{array}{c}\text { Cognitive } \\
\text { Reappraisal } \\
\text { (ERQ) }\end{array}$ & $\begin{array}{c}\text { Expressive } \\
\text { Suppression } \\
\text { (ERQ) }\end{array}$ & $\begin{array}{l}\text { Rumination } \\
\text { (RRS) }\end{array}$ & $\begin{array}{c}\text { Perseverative } \\
\text { Thinking } \\
\text { (PTQ) }\end{array}$ \\
\hline CFQ total & $-.55 * * *$ & $-.39 * * *$ & $-.50 * * *$ & $-.47 * * *$ & $-.54 * * *$ & $-.44 * * *$ & $-.56^{* * *}$ & $-.31 * * *$ & $-.26 * * *$ \\
\hline CFQ Cognitive Control & $-.56 * * *$ & $-.41 * * *$ & $-.52 * * *$ & $-.49 * * *$ & $-.56 * * *$ & $-.46 * * *$ & $-.58 * * *$ & $-.29 * * *$ & $-.25 * * *$ \\
\hline CFQ Cognitive Resources & $-.37 * * *$ & $-.23 * * *$ & $-.28 * * *$ & $-.25 * * *$ & $-.37 * * *$ & $-.22 * * *$ & $-.37 * * *$ & $-.26 * * *$ & $-.20 * * *$ \\
\hline
\end{tabular}

Note. CFQ = Cognitive Flexibility Questionnaire; CFI = Cognitive Flexibility Inventory; COFLEX = Coping Flexibility Questionnaire; ERQ = Emotion

Regulation Questionnaire; RRS = Ruminative Response Scale; PTQ = Perseverative Thinking Questionnaire $* * * p<.001$ 


\section{DISCUSSION}

The present investigation describes a brief self-report measure intended to assess cognitive flexibility in stressful situations and whether disturbances in this ability are related to depressive symptoms. The final version of the CFQ comprised 26 items that addressed two distinct, although overlapping dimensions of cognitive flexibility. The cognitive control dimension addressed primarily cognitive (attentional) control over negative thoughts and emotions elicited by a stressful event. The cognitive resources dimension addressed changes of appraisals (i.e., reappraisal) and the capability of generating multiple alternative coping strategies in the presence of distracting or potentially irrelevant information (e.g., negative thoughts, memories, or emotions). Each of these characteristics has the potential to diminish negative emotional responses.

Preliminary factor analysis and reliability analyses indicated that both the composite CFQ score and the subscale scores were suitable for the measurement of cognitive flexibility and specific dimensions, respectively. Although the composite CFQ score may provide a comprehensive index of cognitive flexibility, the cognitive control and cognitive resources subscales may provide better insight into the processes involved in cognitive flexibility. Indeed, the two subscales of the CFQ are predictive of different processes relevant to stressful events and depressive disorders. Whereas, the cognitive control subscale was particularly aligned with perseverative thinking and rumination, the cognitive resources subscale was more strongly associated with coping flexibility.

Although the CFQ and Cognitive Flexibility Inventory were both designed to assess cognitive flexibility, these measures are predictive of different features relevant to stressful events and depressive disorders. Indeed, whereas the Cognitive Flexibility Inventory reflects the 
type of cognitive flexibility necessary for individuals to challenge and replace maladaptive thoughts, and may thus be useful in assessing the effectiveness of cognitive behavioral techniques for the treatment of depressive disorders (Denis \& Vander Wal, 2010). The CFQ addresses the ability to disengage, or shift attention away, from stressor-elicited negative cognitive and emotional states, and might thereby be more useful in examining the impacts of chronic stressors on dimensions of cognitive flexibility (control and resources).

Ultimately, the goal behind the development of the CFQ was to capture disturbances of cognitive flexibility that may be associated with heightened depressive symptoms that are not singularly captured by other self-report measures. Reduced cognitive flexibility, as assessed by CFQ and subscales, was related to elevated depressive symptoms, even after accounting for emotion regulation, coping flexibility, perseverative negative thinking and rumination, and as such suggests that the CFQ might, in fact, identify important aspects of cognitive flexibility (e.g., cognitive control and cognitive resources) that are aligned with depressive symptoms. However, it remains to be determined whether the CFQ accounts for unique variance, above and beyond, other coping methods, such as positive reframing. 


\section{Study 3B. Cognitive flexibility in relation to cognitive vulnerabilities to depression}

Cognitive vulnerabilities in relation to depression can be expressed in a variety of forms. They may comprise negative self-schemas and dysfunctional attitudes, such as perfectionistic standards and unreasonable expectations of self-worth, which bias cognitive processing (e.g., attention and memory) towards negative, schema-congruent information (Beck, 1967; Beck, Rush, Shaw, \& Emery, 1979). Similarly, they can be manifested as a negative cognitive (attributional) style, characterized by the habitual application of negative inferences concerning the causes and consequences of stressful or negative life events (Abramson et al., 1989; Alloy et al., 1999).

These cognitive vulnerabilities may reflect disturbances in cognitive flexibility as they involve extreme and rigid expectations of self-worth and stereotypical negative inferences of negative life events (Alloy et al., 1999; Beck et al., 1979). Alternatively, dysfunctional attitudes and a negative cognitive style may represent the cognitive content characteristic of depression (e.g., 'I am a failure') whereas cognitive flexibility might facilitate processes facilitating disengagement from negative cognitive content. From this perspective, low levels of cognitive inflexible might favor sustained focus on negative cognitive content (e.g., dysfunctional attitudes and a negative cognitive style), thereby promoting the emergence and maintenance of depressive symptoms. To test this hypothesis, the purpose of Study 2 was to determine whether the effects of dysfunctional attitudes and/or negative cognitive style on depressive symptoms were moderated by individual differences in cognitive flexibility. 


\section{METHODS}

\section{Participants and Procedure}

Participants comprised 171 Carleton University undergraduates $(n=116,67.8 \%$

females), recruited through the university's computerized recruitment system. Participant's mean age was $20.08(S D=3.96)$, and their self-reported ethnicity included Caucasian $(52.6 \%, n=90)$, $\operatorname{Arab}(10.5 \%, n=18)$, Black $(9.9 \%, n=17)$, South Asian $(7.0 \%, n=12)$, Asian $(5.3 \%, n=9)$, Hispanic (3.5\%, $n=6)$, South East Asian $(1.2 \%, n=2)$, Aboriginal $(0.6 \%, n=1)$, and other (e.g., mixed ethnicity, 5.3\%,n=9). Seven individuals (4.1\%) did not report ethnic background. Upon completion of the study, all participants were debriefed and compensated with course credit.

\section{Measures}

Cognitive Flexibility. The 26-item CFQ was used to assess individual differences in cognitive flexibility $(\alpha=.93)$.

Dysfunctional Attitudes. The Dysfunctional Attitudes Scale - short form 1(DAS-SF1; Beevers, Strong, Meyer, Pilkonis, \& Miller, 2007) was used to assess dysfunctional beliefs that are thought to reflect a person's self-evaluation, including concerns about approval from others, prerequisites for happiness, and perfectionist standards. Each of the 9 statements is rated on a scale from 1 (totally agree) to 4 (totally disagree). The total score was computed by subtracting 5 from an item score and summing across all 9 items. Higher score reflects greater dysfunctional attitudes. The internal reliability of the DAS-SF1 in the present study was .81 . 
Negative Cognitive Style. The Cognitive Style Questionnaire-Short Form (CSQ-SF; Meins et al., 2012) was used to assess negative cognitive style. This measure consists of 8 hypothetical scenarios concerning negative life events. For each scenario, the participant is asked to "Imagine themselves vividly in each situation" and respond to 9 items, on a scale ranging from 1 (strongly agree) to 5 (strongly disagree), assessing 5 explanatory dimensions (i.e., internality, globality and stability as well as self-worth and negative consequences). A total negative cognitive style score was computed by reverse scoring appropriate items and then summing across all 64 items. Higher scores reflect a more negative cognitive style. Cronbach's $\alpha$ in the present study was .91 .

Depressive Symptoms. The 21-item Beck Depression Inventory (BDI; Beck, 1961) was used to assess the intensity of depressive symptoms $(\alpha=.91)$.

\section{RESULTS}

Examination of zero-order correlations revealed that reduced cognitive flexibility was associated with significantly greater dysfunctional attitudes $(r=-.31, p<.001)$, greater negative cognitive style $(r=-.28, p<.001)$, and heightened depressive symptoms $(r=-.49, p<.001)$. A hierarchical linear regression was conducted to examine whether cognitive flexibility moderated the relation between dysfunctional attitudes and depressive symptoms. Standardized scores for cognitive flexibility and dysfunctional attitudes were entered on the first step, and the interaction (cross-product of standardized scores) between cognitive flexibility and dysfunctional attitudes was entered on the second step. This analysis revealed significant main effects of dysfunctional attitudes $(b=3.39, S E=.51, p<.001)$ and cognitive flexibility $(b=-2.81, S E=.50, p<.001)$ as well as a significant interaction between dysfunctional attitudes and cognitive flexibility on depressive symptoms $\left(\Delta R^{2}=.09, F(1,165)=28.05, p<.001\right)$. As depicted in Figure 1A, simple 
slopes analysis (Preacher, Curran, \& Bauer, 2006) indicated that greater dysfunctional attitudes were related to heightened depressive symptoms among individuals with lower cognitive flexibility $(b=5.64, S E=.62, p<.001)$, but not for those with greater cognitive flexibility $(b=$ $1.14, S E=.69, p=0.103)$.

A hierarchical linear regression also revealed that depression scores varied with negative cognitive style $(b=2.30, S E=.56, p<.001)$ and cognitive flexibility $(b=-3.57, S E=.55, p<$ $.001)$, as well as the interaction between negative cognitive style and cognitive flexibility $\left(\Delta R^{2}=\right.$ $.04, F(1,167)=11.27, p=.001)$. Follow-up simple slopes analyses, depicted in Figure 1B, revealed that greater negative cognitive style was significantly related to heightened depressive symptoms among individuals with lower cognitive flexibility $(b=3.57, S E=.60, p<.001)$, but not for those with higher cognitive flexibility $(b=1.04, S E=.75, p=0.166)$. Separate hierarchical regressions were conducted to determine whether the cognitive control and cognitive resources subscales of the CFQ moderated the relationship between dysfunctional attitudes and symptoms of depression. In both instances the outcomes were congruent with the findings obtained using the total CFQ scores. In effect, the analyses involving the subscales did not provide information beyond that associated with the total CFQ scores. 

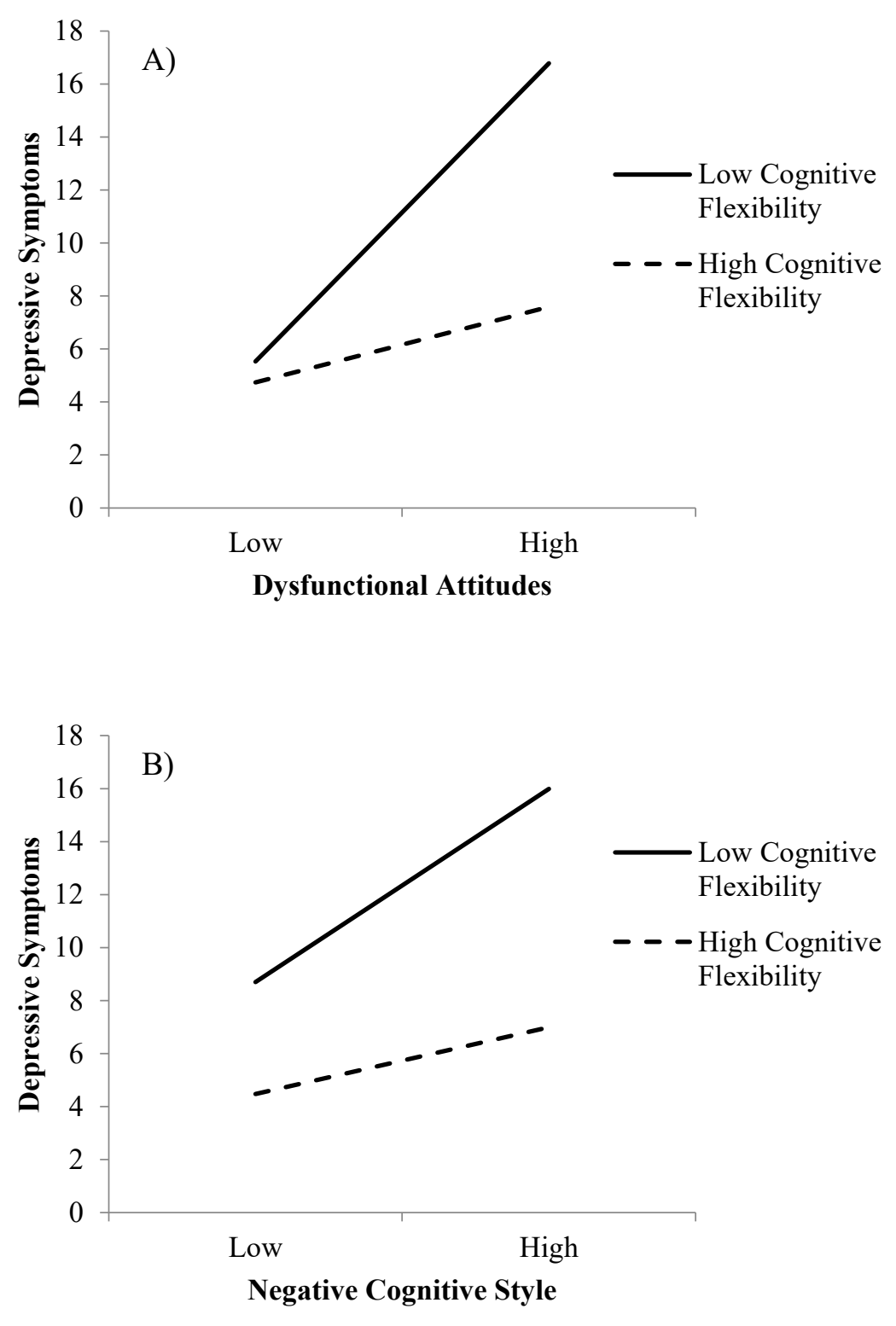

Figure 1. Moderating role of cognitive flexibility in the relation between dysfunctional attitudes and symptoms of depression (A), and negative cognitive style and depressive symptoms (B). 


\section{DISCUSSION}

Dysfunctional attitudes and a negative cognitive style have frequently been observed among depressed individuals, and have been considered to be vulnerability factors for the development of depressive illness (Alloy et al., 1999; Ingram, Miranda \& Segal, 2006). The present findings indicated that the relation between dysfunctional attitudes or a negative cognitive style and depressive symptoms might primarily be apparent among individuals who exhibit reduced cognitive flexibility.

In the presence of stressful or negative life events, dysfunctional attitudes are believed to activate a set of otherwise dormant, negative self-schemas, which are expressed as negative automatic thoughts concerning the self, world, and future (Beck, 1987; Beck et al., 1979). Although activation of negative schemas is likely to elicit a dysphoric state, the impact of such cognitions on depressive pathology might depend on the extent to which they remain active. Similarly, although inferring negative causes and consequences to negative life events (e.g., "It's my fault that this happened, it will have serious implications in all areas of my life, and is likely to happen again") is likely to initially promote a dysphoric state (Abramson et al., 1989), such inferences may not invariably lead to depressive illness. Instead, the impact of such negative inferences might depend upon whether they are modified or maintained over time.

A high degree of cognitive flexibility, and in particular greater cognitive control, may facilitate processes that can alleviate negative affect. In contrast, disturbed cognitive flexibility might contribute to a preoccupation and exacerbation of negative cognitive content (e.g., 
negative self-schemas or negative inferences), thereby maintaining or enhancing negative mood. Consistent with this suggestion, it has reported that the relation between negative cognitive styles and depressive symptoms was only evident among individuals high in rumination (Robinson \& Alloy, 2003), which has been associated with cognitive inflexibility (Davis \& Nolen-Hoeksema, 2000; see also Study 1). 
Study 3C. Cognitive flexibility in relation to stressor appraisals, coping style, and depressive affect

How individuals appraise and cope with stressful events plays an important role with respect to vulnerability or resilience to depressive pathology. For instance, depressed individuals generally appraise stressful events as being more threatening (Folkman \& Lazarus, 1986) and frequently perceive themselves as lacking personal control over such events (Abramson et al., 1989). These appraisals are often accompanied or followed by increased utilization of emotionfocused and avoidant coping strategies at the expense of problem-oriented coping methods (Matheson \& Anisman, 2003; Whatley, Foreman, \& Richards, 1998). In contrast, cognitive flexibility has been associated with more problem-focused coping and social support seeking, and less engagement in emotion-focused and avoidant coping methods, including wishing thinking (Dennis \& Vander Wal, 2010). Through effects on appraisals and coping, cognitive inflexibility may favor the development of depressive symptoms in response to stressful experiences. Study 3 examined whether the relation between reduced cognitive flexibility and heightened depressive symptoms was mediated by negative appraisals of stressful events, particularly those encompassing threat and control, as well as by the persistent use of particular coping styles.

\section{METHODS}

Participants 
Participants comprised 368 Carleton University students ( $n=288,78.3 \%$ females), with a mean age of $19.53(S D=1.59)$. Self-reported ethnicity included Caucasian $(70.9 \%, n=261)$, Asian $(6.8 \%, n=25)$, Black $(6.0 \%, n=22)$, Arab (5.2\%, $n=19)$, South Asian $(4.1 \%, n=15)$, Latin American $(2.2 \%, n=8)$, South East Asian $(1.6 \%, n=6)$, Aboriginal $(0.3 \%, n=1)$, and other (e.g., mixed ethnicity, 3.0\%,n=11).

\section{Procedure}

As in Study 1, participants were recruited through the university's online computerized recruitment system, and participated in an online survey-based study. Following informed consent, participants were asked to reflect on a recent and personally meaningful academic situation that they found stressful, and to evaluate this situation on several stress appraisal dimensions (e.g., threat, challenge, and controllability). The central theme for the majority of participants was “overwhelmed with school work (e.g., exams and term papers)". Several additional measures where then completed which assessed cognitive flexibility, coping style, and current symptoms of depression. Upon completion of the study, participants were debriefed and compensated with course credit.

\section{Measures}

Cognitive Flexibility. The CFQ was used to assess cognitive flexibility, which once more exhibited high internal reliability (see Table 5).

Stressor Appraisals. The Stress Appraisal Measure (SAM; Peacock \& Wong, 1990) was used to assess several dimensions of stress appraisals in response to the academic stressor, including, perceptions of threat (e.g., "How threatening is the situation?"), challenge ("How eager are you to tackle this problem"), centrality ("Does this situations have important 
consequences for you?"), control-by-self (“Do you have the ability to do well in this situation?"), control-by-others (“Is there someone or some agency you can turn to for help if you need it?"), and uncontrollable-by-anyone ("Is the situation totally hopeless?"). The response format consisted of a five-point rating scale ranging from 1 (not at all) to 5 (a great amount). Scale scores for each appraisal dimension were calculated by obtaining the mean rating for items comprising each scale.

Coping Style. Coping style was assessed using the Survey of Coping Profile Endorsement (SCOPE; Matheson \& Anisman, 2003). The SCOPE is a 50-item measure assessing the frequency of endorsement of 13 coping strategies. For each item, respondents were asked to indicate the extent to which they had demonstrated each of the behaviours as a way of dealing with stressors in recent weeks using a scale of 0 (Never) to 4 (Frequently). Scores were computed by taking the mean across all relevant items for each form of coping. Based on previous use of the SCOPE (Matheson \& Anisman, 2003) and a principal component analysis, three broad clusters of coping were examined. Specifically, problem-focused coping comprised problem-solving, cognitive restructuring, active distraction, humour, and social support seeking; emotion-focused coping included rumination, emotional expression, emotional containment, other-blame, and self-blame; and avoidant coping comprised avoidance, wishful thinking, and passive resignation.

Depressive Symptoms. The 21-item BDI (Beck, 1961) was again used to assess the intensity of depressive symptoms.

RESULTS 
Consistent with Study 1 and 2, greater cognitive flexibility was associated lower levels of depressive symptoms $(r=-.58, p<.001)$. Furthermore, in response to an academic stressor (e.g., being overwhelmed with school work), individuals who reported greater cognitive flexibility appraised the event as less threatening $(r=-.37, p<.001)$, more challenging $(r=.29, p<.001)$, perceived greater control by the individual $(r=.27, p<.001)$, and as less uncontrollable by anyone $(r=-.13, p=.011)$. In contrast, cognitive flexibility was not related to perceptions of centrality $(r=-.09, p=.099)$ or whether the situation was controllable by others $(r=.02, p$ $=.676)$.

A multiple mediation analysis, using 5000 bootstrap sample and $95 \%$ bias corrected confidence intervals (Preacher \& Hayes, 2008), was conducted to examine whether specific dimensions of stressor appraisals mediated the relation between cognitive flexibility and symptoms of depression. In this regard, only the appraisals that were significantly associated with cognitive flexibility were considered in the analysis. As depicted in Figure 1, when considering all four appraisal dimensions, perceptions of threat (95\% C.I.: -.90, -.12) and uncontrollability $(95 \%$ C.I.: -.60, -.05) significantly mediated the relation between cognitive flexibility and symptoms of depression. Although greater cognitive flexibility was also related to higher challenge and control-by-self appraisals, they did uniquely not serve as mediators in the relation between cognitive flexibility and depressive symptoms challenge (95\% C.I.: -..42, .17 and 95\% C.I.: -.67, .09, respectively). 


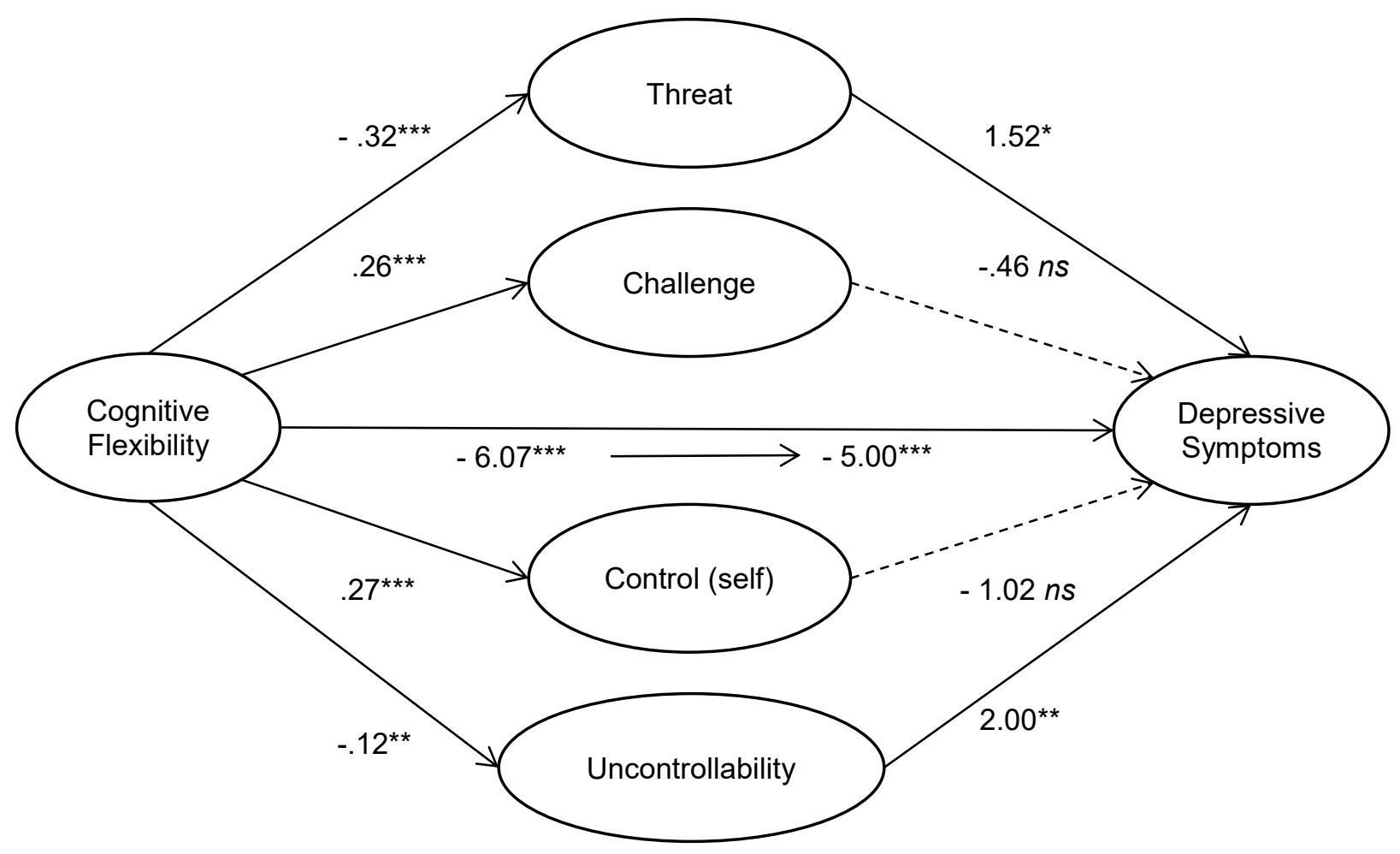

Figure 1. Multiple mediation analysis examining the mediating effect of threat and uncontrollability appraisals in the relationship between cognitive flexibility and depressive symptoms. Note $* p<.05 * * p<.01 * * * p<.001$

Additionally, individuals who were more cognitively flexible, reported greater endorsement of problem-focused coping $(r=.33, p<.001)$ as well as lower utilization of both emotion-focused $(r=-.54, p<.001)$ and avoidant coping $(r=-.31, p<.001)$. A second multiple mediation analysis, illustrated in Figure 4, indicated that both problem-focused (95\% C.I.: -1.10, $-.41)$ and emotion-focused (95\% C.I.: $-3.39,-1.89)$ coping significantly mediated the relation between cognitive flexibility and depressive symptoms. Although greater cognitive flexibility was related to lower avoidant coping, when considering problem- and emotion-focused coping in the model, avoidant coping $(95 \%$ C.I.: $-.47, .14)$ did not serve as a unique mediator in the 
relationship between cognitive flexibility and symptoms of depression. To examine whether these mediating effects were unique to specific dimensions of cognitive flexibility, several additional multiple mediation analyses were conducted using the cognitive control and cognitive resources subscales of the CFQ. Once again, these analyses indicated that the subscales did not provide meaningful information beyond that obtained using the total CFQ scores. Thus, only the analyses involving the total scores are provided here.

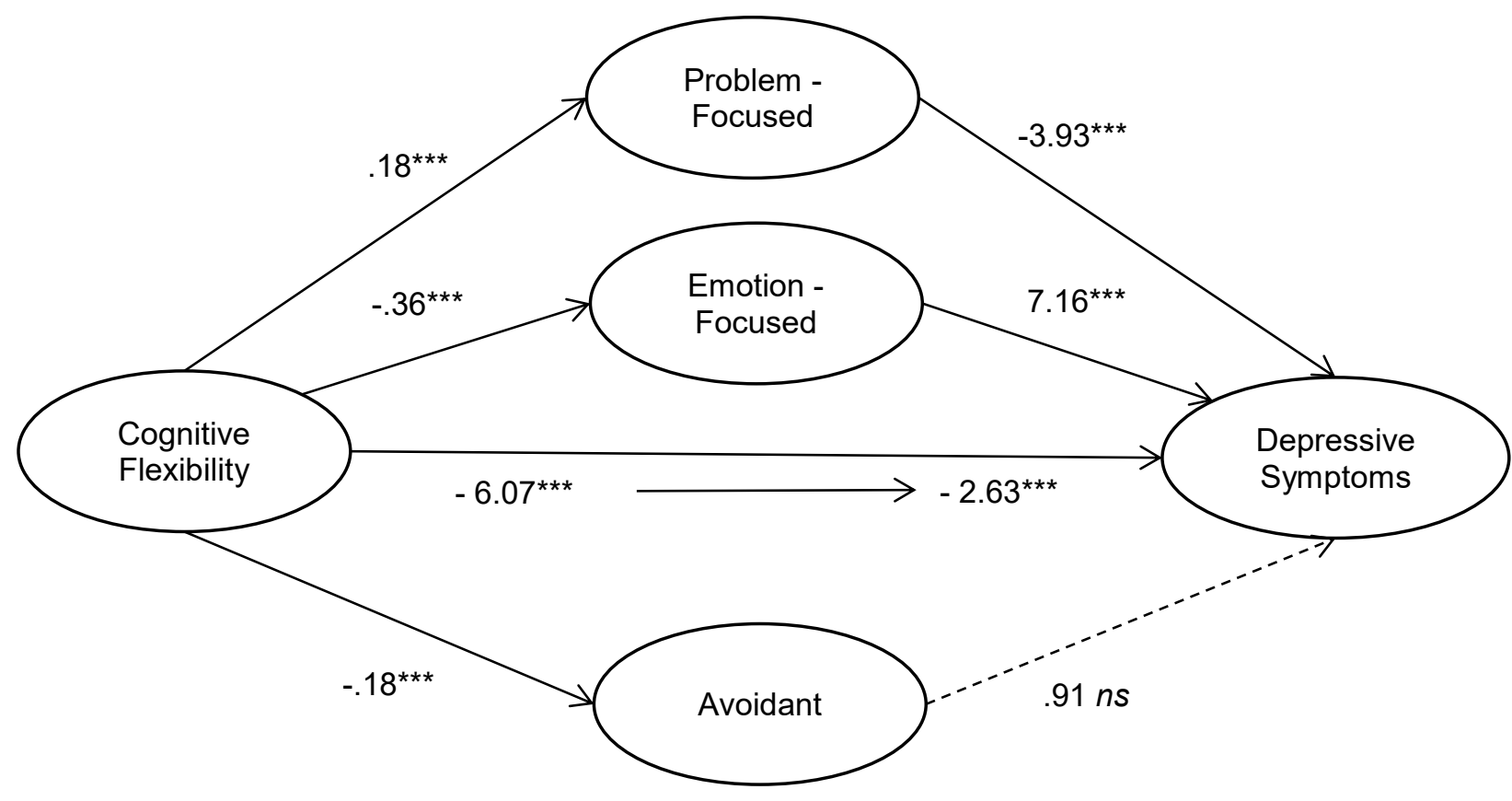

Figure 2. Multiple mediation analysis examining the mediating effect of problem- and emotionfocused coping in the relationship between cognitive flexibility and depressive symptoms. Note $* * * p<.001$ 


\section{DISCUSSION}

It appeared that the link between reduced cognitive flexibility and heightened depressive symptoms occurred through negative appraisals concerning stressful events, notably those involving perceived threat and uncontrollability. These findings were not unexpected as the perceived lack of control over life events, as well as greater perceived threat have long been considered fundamental in the evolution of depressive symptomatology (Abramson et al., 1989; Beck, 1987; Benassi, Sweeney, \& Dufour, 1988; Folkman \& Lazarus, 1986). In addition, as mentioned earlier, a central aspect of cognitive flexibility is reappraisal, or the ability to view a stressful situation from multiple perspectives or by reinterpreting a negative situation as being more positive. In this regard, although a stressful situation might initially elicit negative appraisals (e.g., perceptions of threat and uncontrollability), individuals with greater cognitive flexibility might be more inclined to modify their initial appraisals (e.g., reinterpreting these events as challenge and controllable), leading to the attenuation of negative emotional responses. In contrast, individuals with reduced cognitive flexibility would be more like to automatically assign negative appraisals and display difficulties in changing them over time, resulting in the maintenance or exacerbation of negative affect.

As previously observed using the CFI (Dennis \& Vander Wal, 2010), greater cognitive flexibility assessed by the CFQ, was associated with lower utilization of emotion-focused (e.g., rumination and blame) and avoidant (e.g., passive resignation and wishful thinking) coping, and greater endorsement of problem-focused coping methods, including problem-solving and cognitive restructuring. The data, although correlational, were in line with the view that reductions in cognitive flexibility might be related to depressive symptoms is through the persistent engagement in less effective coping. In this respect, individuals with limited cognitive 
flexibility may become preoccupied with distracting emotional information and mood states, thus hindering their ability to generate multiple effective coping strategies, and contributing to the endorsement of a limited number of coping methods. As a result, individuals who are less cognitively flexible may rely more on emotion-focused and avoidant coping at the expense of problem-focused coping methods.

\section{GENERAL DISCUSSION}

The present studies examined the links between cognitive flexibility in the context of stressful experiences and symptoms of depression. In doing so, a brief self-report measure (the CFQ) was developed that captured several indices of cognitive flexibility relevant to stressful situations. The cognitive control dimension was designed to capture fundamental processes involved in cognitive flexibility, including attentional control and attentional set shifting in response to negative thoughts and emotions. The cognitive resources dimensions of the CFQ was designed to address more complex, or higher order, forms of cognitive flexibility, including the ability to modify appraisals (i.e., reappraisal) and to generate multiple alternative coping strategies in the presence of negative thoughts and emotions. The CFQ appeared to address aspects of cognitive flexibility that are not captured by other self-report measures, including the Cognitive Flexibility Inventory.

Whereas dysfunctional attitudes and a negative cognitive style represent the cognitive content characteristic of depression (Robinson \& Alloy, 2003), cognitive flexibility might favor processes that facilitate disengagement from negative cognitive content. Disturbances in cognitive flexibility, however, might engender a different form of cognitive vulnerability that contributes to the maintenance of negative cognitive and emotional states. In this regard, depressive symptoms might vary with both the presence of negative cognitive content (e.g., 
dysfunctional attitudes and a negative cognitive style) and disturbed cognitive processes (e.g., cognitive flexibility or cognitive control). There appeared to be several ways through which disturbed cognitive flexibility might be associated heightened depressive symptoms. In particular, individuals who reported reduced cognitive flexibility more readily assigned negative appraisals to a personally meaningful stressor (in this case an academic challenge) and endorsed more emotion-focused and diminished problem-focused coping, which were linked to depressive symptoms.

There were several limitations regarding the present findings. As the studies were crosssectional, it is not possible to conclude that reduced cognitive flexibility contributes to the emergence and maintenance of depressive pathology. Chronic stressors have been associated with disturbed cognitive flexibility (Liston, McEwen, \& Casey, 2009), but prospective studies are necessary to determine whether reductions in cognitive flexibility are predictive of subsequent elevations of depressive symptoms. Furthermore, the present series of studies comprised undergraduate students who in the main did not display clinically significant symptoms of depression. As such, the generalizability of the current findings may be somewhat limited. Clinical samples would be of obvious benefit in assessing the link between cognitive flexibility and depressive features, and could also be used to determine whether interventions aimed at enhancing cognitive flexibility would be accompanied by alleviation of depressive symptoms.

Despite these limitations, the present findings point to ways in which cognitive flexibility might be expressed in the context of stressful situations, and that reductions in this ability are associated with heightened depressive symptoms. The brief self-report measure provided may be useful in identifying certain aspects of cognitive flexibility that might be disturbed among 
stressed or depressed individuals, including cognitive control and cognitive resources, which could be potential targets for the development of intervention strategies. 


\title{
CHAPTER 4
}

\section{Emotional and neuroendocrine responses following an acute psychosocial stressor: moderating role of cognitive flexibility}

\begin{abstract}
Disturbances in cognitive control may underlie the difficulties of emotional regulation that are characteristic of depressive disorders. However, it is less certain how disturbed cognitive control leads to prolonged emotional and neuroendocrine responses following a stressor. The present investigation examined whether the intensity and duration of emotional and cortisol responses elicited by the Trier Social Stress Test (TSST) were mediated by stressor appraisals, and whether this relation was moderated by cognitive flexibility, as assessed by the Cognitive Flexibility Questionnaire (CFQ). Among undergraduate students $(N=44)$, greater perceived threat and uncontrollability mediated the relation between stressor condition increased negative affect immediately post-stressor, and this relation was moderated by individual differences in cognitive control and resources. Specifically, the TSST was appraised as being more threatening by individuals with low cognitive control, which was related to increased negative affect. Similarly, the TSST was interpreted as uncontrollable by individuals with low, but not high, cognitive resources, which was then accompanied by elevated negative affect. At 40 minutes following the stressor, the relation between stressor condition and negative affect, which was mediated by greater perceived threat and uncontrollability, was once again moderated by cognitive control and resources. However, at 40 minutes following the stressor TSST was still appraised as threatening and uncontrollable, but these appraisals were more strongly associated with negative affect among individuals with low cognitive control and cognitive resources. Finally, the relation between stressor condition and plasma cortisol levels was mediated by
\end{abstract}


greater perceived uncontrollability, but among individuals with low cognitive resources. In essence, differences in cognitive flexibility might play an important role in regulating the emotional and neuroendocrine responses elicited by an acute stressor, although the processes involved in these effects might vary over the course of a stressful encounter. 


\section{INTRODUCTION}

Depressive disorders have been associated with disturbances in cognitive (executive) control, with the most notable deficits comprising working memory, response inhibition, and setshifting (Austin et al., 2001; Grant et al., 2001; Trivedi \& Greer, 2014). These disturbances have not only been observed during the course of a depressive episode, but were also evident during recovery from the illness (Paelecke-Haberman et al., 2005; Paradiso et al., 1997), and could potentially contribute to depressive relapse (Alexopoulos et al., 2005; Paelecke-Haberman et al., 2005). Cognitive control processes my influence the flexibility in the ability to adapt to continuously changing environmental demands and, thus, impairments in these abilities might contribute to rigid and automatic responses, ultimately compromising goal-directed behavior.

Cognitive control processes also play a role in the regulation of emotions (Gotlib \& Joormann, 2010; Joormann, 2010; Ochsner \& Gross, 2005; Ochsner, Silvers, \& Buhle, 2012). In the context of depressive disorders, disturbed or reduced cognitive control abilities can hinder an individual's ability to disengage from negative emotional material, resulting in negative thinking, and the maintenance and exacerbation of corresponding negative mood states (Joorman, Yoon, \& Gotlib, 2007). Reduced cognitive control can also favor the utilization of ineffective emotion regulation strategies, such as rumination, and undermining an individual's ability to engage in more effective strategies, including cognitive reappraisal (Joorman, 2010; Koster et al., 2011).

In line with the view that disturbances in cognitive control might promote the emergence and maintenance of depressive states (Joorman, 2010), a self-report measure (i.e., the Cognitive Flexibility Questionnaire, CFQ) was developed to examine how cognitive flexibility might be associated with elevated depressive symptoms, and the processes through which this might occur (see Chapter 3). The CFQ, as seen earlier, comprises two distinct, yet overlapping, dimensions 
that assess processes (e.g., attention, appraisal and reappraisal, and coping) through which cognitive flexibility might be expressed in stressful situations. The cognitive control dimension of the CFQ addresses the extent to which an individual can control intrusive and repetitive (primarily negative) thoughts and emotions ordinarily elicited by a stressful situation. As assessed by this dimension of the CFQ, individuals with greater cognitive control find it relatively easy to shift their attention away from negative cognitive and emotional states, whereas those with low cognitive control are readily distracted by negative emotional states and find it difficult to limit repetitive negative thinking and the maintenance of negative emotions. The second dimension of the CFQ, the cognitive resources dimension, assesses an individual's ability to engage in a set of deliberate (effortful) behaviours that can facilitate an appropriate, or favorable, appraisal of a stressful situation and the flexible selection of a broad range of coping strategies. As measured by this dimension of the CFQ, individuals with greater cognitive resources tend to approach stressful situations from multiple perspectives prior to responding, manage negative thoughts and emotions by reframing or reappraising stressful situations, and generate multiple and alternative coping strategies prior to selecting a response. Individuals with low cognitive resources, by contrast, tend to respond more impulsively, automatically, and are more reactive in their cognitive, emotional, and behavioral responses.

As described in Chapter 3, cognitive flexibility (including cognitive control and cognitive resources dimensions) moderated the relationship of negative cognitive styles and symptoms of depression. Specifically, higher levels of dysfunctional attitudes (i.e., a set of unrealistic attitudes and beliefs concerning the self, the world, and the future) as well as a more negative cognitive style (i.e., the tendency to attribute that causes and consequences of negative life events to internal, stable, and global sources) were related to heightened depressive symptoms primarily 
among individuals who reported low levels of cognitive flexibility. Furthermore, the relationship between decreased cognitive flexibility and heightened depressive symptoms was mediated by negative stressor appraisals (e.g., appraising a stressful event as threatening and less controllable), greater engagement of emotion focused coping (e.g., rumination), and diminished use of problem-focused methods (e.g., cognitive restructuring).

In light of these relations, the purpose of the present investigation was to examine whether individual differences in specific dimensions of the CFQ (i.e., cognitive control and cognitive resources) moderated the effects of the Trier Social Stress Test (TSST) on the intensity of negative emotional responses evident immediately and 40 minutes following the stressor. It was hypothesized that the mediating role of stressor appraisals (e.g., perceptions of threat, uncontrollability, and control-by-self, the latter comprising the evaluation of an individual's coping resources or their ability to do well in a stressful situation) in the relation between stressor condition and emotional responses would be moderated by cognitive flexibility.

In addition to precipitating negative emotional states, stressful events are often associated with the activation of the hypothalamic-pituitary-adrenal (HPA) axis, culminating with the release of cortisol from the adrenal glands (Smith \& Vale, 2009). In the short term, the secretion of cortisol serves an adaptive purpose in mobilizing essential (cognitive and biological) resources to deal with a stressor encounter (McEwen, 1998). However, excessive or prolonged release of this glucocorticoid can contribute to excessive "wear and tear" on multiple biological systems (allostatic overload), resulting in diminished cognitive functioning and the evolution of pathological outcomes, including depressive illness (McEwen, 2004; McEwen \& Morrison, 2013). Thus, the ability to regulate HPA axis activity effectively, along with other stress 
response systems, might be an important mechanism involved in an individual's resilience to psychopathology.

It is becoming increasingly apparent that cognitive control processes might not only serve to regulate emotions, but can also influence the physiological responses precipitated by stressful events (Compton, Hofheimer, \& Kazinka, 2014; Shields et al., 2016). For example, cognitive reappraisal, which involves multiple cognitive control processes (Ochsner et al., 2012), moderated the release and duration of cortisol as well as heart rate variability in the presence of a stressful event (Denson et al., 2014; Gaab et al., 2003; Jamieson et al., 2012). In contrast, following an acute psychosocial stressor, the tendency to utilize rumination, which has been associated with diminished cognitive control (Koster et al., 2011), was related to delayed cortisol recovery (Stewart et al., 2013; Zoccola, Quas, \& Yim, 2010). Thus, it was hypothesized that cognitive flexibility (i.e., cognitive control and cognitive resources) would moderate the impact of the TSST on plasma cortisol responses.

\section{METHODS}

\section{Participants}

The present study comprised male $(n=20)$ and female $(n=24)$ undergraduate students from Carleton University ranging in age from $18-26$ years $\left(M_{\text {age }}=20.17, S D=2.25\right)$. Participants represented an ethnically diverse sample consisting of White $(40.9 \%, n=18)$, South Asian $(15.9 \%, n=7)$, Arab/West Asian (9.1\%, $n=4)$, South East Asian $(6.8 \%, n=3)$, Latin American/Hispanic $(6.8 \%, n=3)$, Black $(4.5 \%, n=2)$, Asian $(2.3 \%, n=1)$, Aboriginal $(2.3 \%, n$ $=1)$, and other $(9.1 \%, n=4)$. None of the participants reported a current physical illness/condition, and five reported being currently diagnosed with an anxiety or depressive 
disorder. None of the participants were taking anti-inflammatory, anti-anxiety, or anti-depressant medications. Of the female participants, 10 were taking an oral contraceptive.

\section{General Procedure}

Participants were recruited through the university's computerized recruitment system in which they completed a brief on-line pre-screening questionnaire that determined their eligibility for the laboratory session. The on-line pre-screening questionnaire assessed the presence of several exclusion criteria, including medical conditions or medications that may influence hormone functioning as well as potential issues surrounding blood collection (e.g. a fear of needles, previous history of nausea or fainting during blood collection, or difficulty with veins). Participants who met eligibility criteria were randomly assigned to either the TSST $(n=21)$ or control $(n=23)$ condition and invited to participate in the laboratory session.

\section{Laboratory Session}

All procedures in this study were approved by the Carleton University Ethics Committee for Psychological Research. Laboratory sessions were conducted between 1300 and $1730 \mathrm{hr}$, and all participants were asked to not eat, drink (with the exception of water), smoke, or exercise for at least an hour before arriving to the session.

Following the introduction to the study and the acquisition of written informed consent, participants were given 15 minutes to habituate to the laboratory environment. During this time, they completed several questionnaires concerning demographic information (e.g., age, gender, and ethnicity) as well as the presence of any physical or psychological illnesses and current medication. At 15 the minute mark, a registered nurse inserted a catheter into the participants' 
non-dominant arm for blood collection. Participants were then asked to relax for an additional 10 minutes.

Following the relaxation period, participants in the stress condition were instructed that they would be given 5 minutes to prepare for an "employment task" comprising a five-minute speech followed by a five-minute mental arithmetic task in front of a panel of 3 graduate student judges. A video camera was also set up and the participants were told their performance would be videotaped and later evaluated. Immediately following the 5 minute preparatory period, participants in the stressor condition underwent the TSST. Participants in the control condition were also asked to complete an employment task, but this comprised writing their strengths and past work/volunteer experience on a form.

Following the TSST or control task, participants were given 10 minutes to complete measures of stressor appraisals and current mood. At 10 minutes, participants completed two working memory tasks which were part of a parallel study. These tasks took approximately 30 minutes to complete. Following the completion of the working memory tasks, participants completed the same stressor appraisals and mood measures as well as a measure assessing cognitive flexibility. During this time (at 45 minutes post-TSST), the catheter was removed and, once the questionnaires were completed, participants were debriefed.

\section{Measures}

Stressor appraisals. The Stress Appraisal Measure (SAM; Peacock and Wong, 1990) was used to assess differences in stressor appraisals between the TSST and control groups (i) immediately post-TSST and (ii) 40 minutes post-TSST (30 minute following the initial appraisal). For the purposes of the present investigation, only perceptions of threat and 
uncontrollability (i.e., the extent to which the outcome of a stressful situation in uncontrollable or unresolvable) were of interest. Scores for each appraisal dimension were computed by taking the mean across all relevant items. These items were measured on a five-point scale ranging from 1 (not at all) to 5 (extremely), with higher scores indicating greater levels of perceived threat and uncontrollability. The internal consistencies of stressor appraisals immediately following the stressor/control condition were: threat $(\alpha=.73)$ and uncontrollability $(\alpha=.92)$. The internal consistencies of stressor appraisals 40 minutes following the stressor/control condition were: threat $(\alpha=.78)$ and uncontrollability $(\alpha=.92)$.

Mood. The Positive and Negative Affect Schedule - Expanded Version (PANAS-X; Watson \& Clark, 1999) was used to assess the intensity of emotional responses (i) immediately post-TSST and (ii) 40 minutes post-TSST (30 minutes following the initial emotional response). In the present study, only the subscales for positive and negative affect were utilized. Responses ranged on a six-point scale from 0 (not at all) to 6 (extremely), with higher scores indicating greater intensity of positive or negative affect. The internal consistency of items for emotions immediately post-stressor/control condition was .85 and .93 for positive and negative affect, respectively. The internal consistency of items for these affective states 40 minutes poststressor/control condition was .88 and .96 positive and negative affect, respectively

Cognitive Flexibility. The 26-item Cognitive Flexibility Questionnaire was used to assess individual differences in two dimensions of cognitive flexibility, including cognitive control ( $\alpha=$ $.95)$ and cognitive resources $(\alpha=.89)$.

\section{Blood Collection}

Blood samples were collected continuously using a Dakmed ambulatory pump, at a low draw rate $(2.08 \mathrm{~mL} / 5 \mathrm{~min})$ into chilled EDTA coated vacutainer tubes. For each time-point of 
interest, samples were taken at an increased draw rate $(6.9 \mathrm{~mL} / 5 \mathrm{~min})$. These time-points included: i) immediately before the TSST or control task (25 minutes after the participant's arrival to the study session), and then at 5, 15, 30, and 45 minutes post-task. Approximately $2.75 \mathrm{~mL}$ of plasma was collected for each of the time-points of interest. Following collection, plasma samples were centrifuged for 15 minutes at $4^{\circ} \mathrm{C}$ and $2100 \mathrm{~g}$, and were immediately aliquoted into Eppendorf tubes and frozen at $-80^{\circ} \mathrm{C}$.

Of the 44 participants, there were 3 individuals from whom blood samples could not be obtained due to complications with small veins.

\section{Plasma Cortisol}

Plasma cortisol was determined in duplicate by a radioimmuno assay (RIA) using the ${ }^{125} \mathrm{I}$ kit obtained from ICN Biomedicals Inc., Irvine, CA. The assays were performed according to the manufacturer's instructions. The intra-assay variability was less than $8 \%$ and the minimum detectable concentration was $0.17 \mu \mathrm{g} / \mathrm{dL}$.

\section{Statistical Analyses}

Statistical analyses were performed using SPSS for Windows 18.0 (SPSS Science, Chicago, IL). Changes in stressor appraisals and mood over time as a function of experimental condition (stressor vs control) were analyzed using a using a 2 (Stressor Condition) x 2 (Time: 2 time-points) mixed measures ANOVA with Time serving as the within-group factor. Follow-up comparisons comprised t-tests with a Bonferroni correction to maintain the alpha level at 0.05. Similarly, changes of plasma cortisol levels as a function of experimental condition were analyzed using a 2 (Stressor Condition) x 5 (Time: 5 time-points) mixed measures ANOVA. Follow-up comparisons, once again, comprised t-tests with a Bonferroni correction to maintain 
the alpha level at 0.05 . In addition, area under the curve with respect to increased cortisol (AUCi) (Pruessner et al., 2003) was computed to examine the relation between a cumulative cortisol response and stressor appraisals and mood state. Mediation analyses were conducted using the PROCESS add-on to SPSS provided by Preacher \& Hayes (Preacher \& Hayes, 2008; Hayes, 2013), using 5000 bootstrap samples and 95\% Bias Corrected Confidence Intervals (C.I.). Hierarchical linear regression analyses were used to examine the moderating role of cognitive flexibility in the relation between stressor condition and stressor appraisals, mood, and cortisol response. Significant moderations were followed up using a web utility for simple slopes (Preacher et al., 2006). Moderated mediation analyses were conducted using bootstrapping procedures and confidence intervals were based on 5000 resamples (Preacher et al., 2007).

\section{RESULTS}

\section{Changes in emotional state over time as a function of stressor condition}

A mixed measures ANOVA revealed a main effect of Time $F(1,42)=22.91, p<.001$ and a Time $\mathrm{x}$ Stressor Condition interaction $F(1,42)=8.86, p=.005$ for the intensity of negative affect following exposure to the TSST (control condition). As illustrated in Figure 1A, immediately post-stressor, individuals who had undergone the TSST reported significantly greater levels of negative affect in comparison to those who were in the control condition, $t$ (42) $=2.94 p=.005$. However, 40 minutes following the stressor, individuals in the TSST and control conditions no longer differed in this regard, $t(42)=-1.41 p>.05$. Moreover, among individuals exposed to the TSST condition, the intensity of negative affect declined from immediately poststressor to $40 \mathrm{~min}$ afterwards, $t(42)=5.392 p<.001$, but did not change among those in the control condition, $t(42)=1.31 p>.05$. 
As shown in Figure 1B, although a main effect of Time, $F(1,42)=11.57, p<.001$, was observed for positive affect, the Time $\mathrm{x}$ Condition interaction, $F(1,42)=1.56, p>.05$, was not significant. As depicted in Figure 1B, a small decline of positive effects was apparent over time, being marginally more pronounced among individuals in the stressor condition.
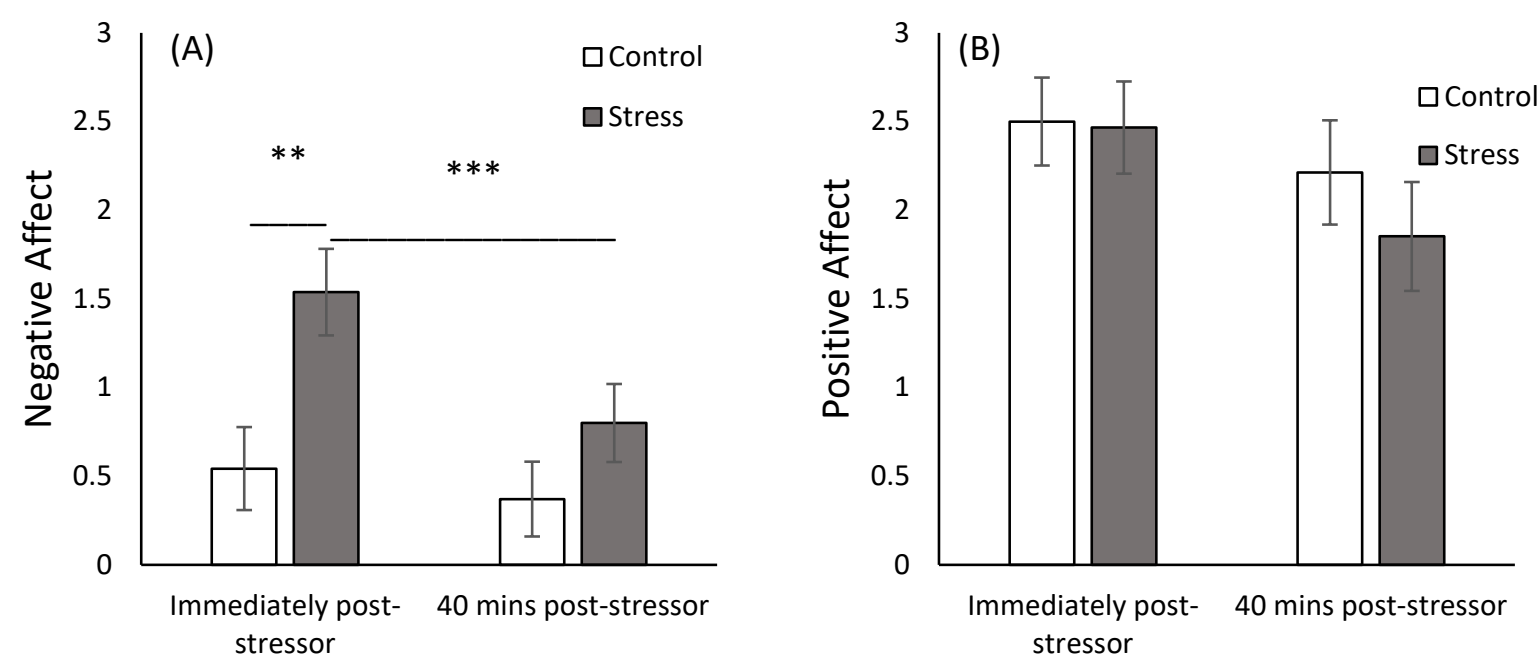

Figure 1. Mean differences ( \pm S.E.M.) between the stressor and control groups in the intensity of negative (A) and positive (B) affect over time. ${ }^{* * *} p<.001, * * p<.01$

Stress appraisals over time as a function of stressor condition

A mixed measures ANOVA of perceived threat revealed no significant main effect of Time, $F(1,42)=2.48, p>.05$, or the Time $\mathrm{x}$ Condition interaction, $F(1,42)=2.14, p>.05$, Similarly, no significant main effect of Time, $F(1,42)=1.39, p>.05$, or Time $\mathrm{x}$ Condition interaction, $(F(1,42)=1.22, p>.05$, was observed for perceived uncontrollability. As illustrated in Figures 2A and B, immediately and 40 min following the stressor, the TSST was appraised as more threatening and uncontrollable than the control condition. 

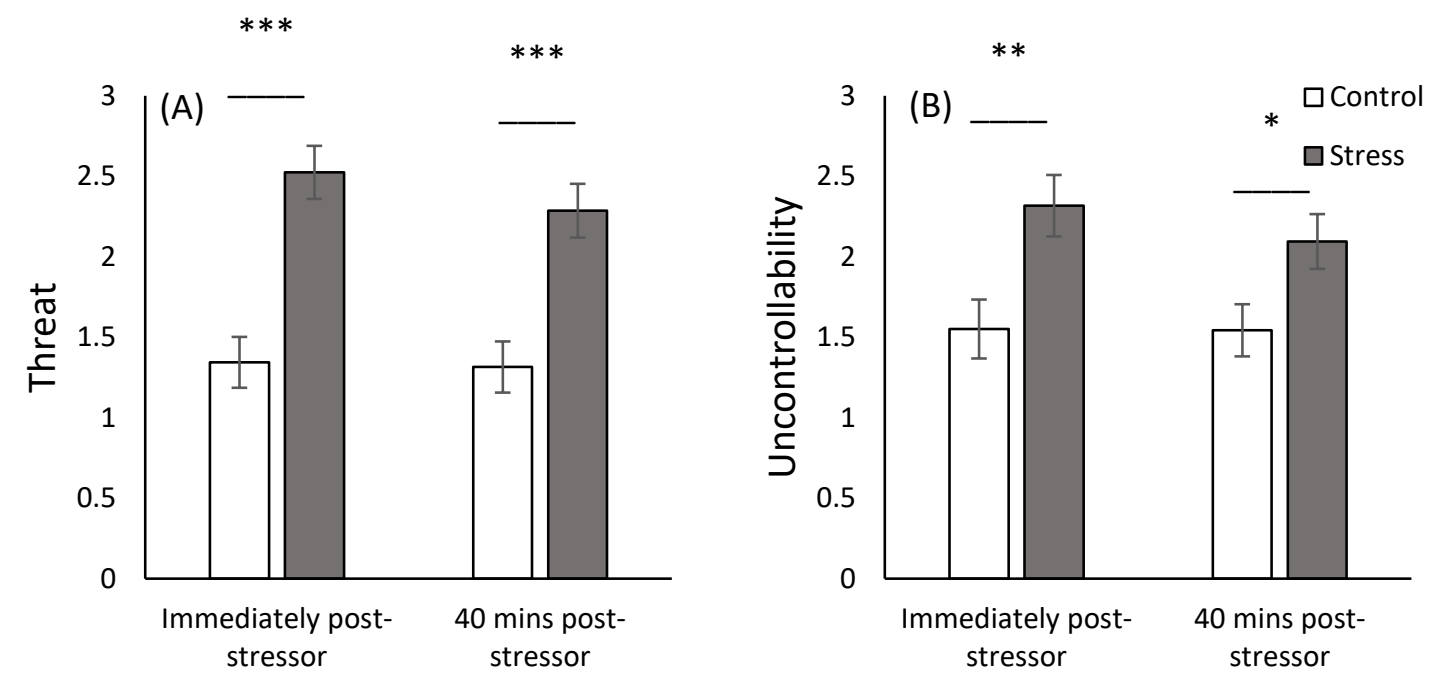

Figure 2. Mean differences ( \pm S.E.M.) between the stressor and control groups in perceived threat (A) and uncontrollability (B) over time. ${ }^{* * *} p<.001, * * p<.01, * p<.05$.

The mediating role of stressor appraisals in the relation between stressor condition and negative affect was time-dependently moderated by cognitive flexibility

Immediately following the TSST (or control) condition, a moderated mediation analysis revealed that the relationship between stressor condition and negative affect was mediated by threat appraisals, and these relations were moderated by individual differences in cognitive control. As illustrated in Figure 3, the TSST was appraised as more threatening among individuals with low cognitive control, and heightened perceived threat was then accompanied by an immediate increase in negative affect. 


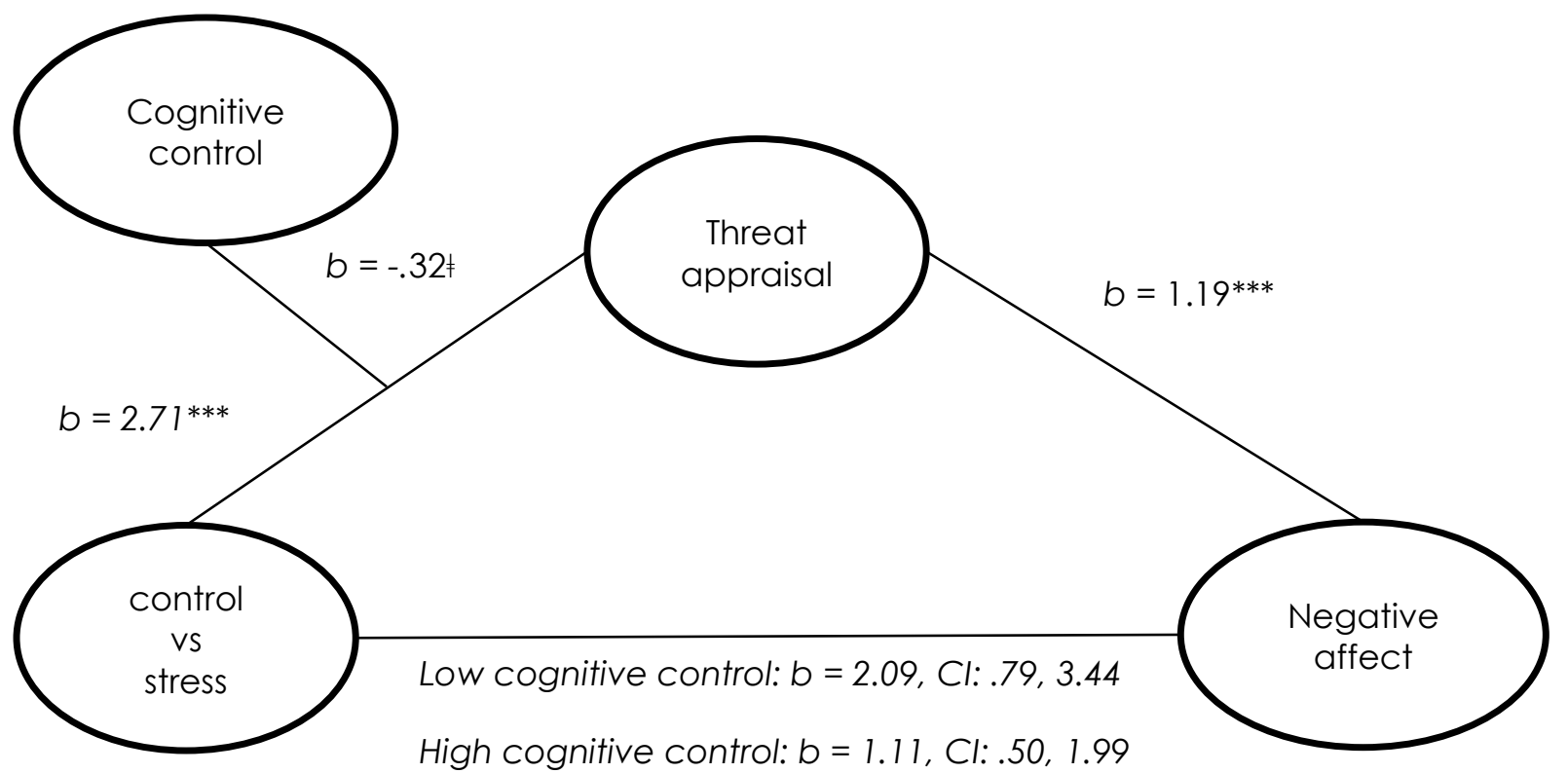

Figure 3. Immediately post-stressor, the mediating role of threat appraisal in the relation between stressor condition and negative affect was moderated by differences in cognitive control. Note: low cognitive control $=1 S . D$. below the mean, high cognitive control $=1 S . D$. above the mean.

$* * * p<.001, \neq p=.07$

Similarly, immediately following the TSST (or the control) condition, the relationship between stressor condition and negative affect was also mediated by perceived uncontrollability, but in this case these relations were moderated by individual differences in cognitive resources (but not cognitive control). As shown in Figure 4, the TSST was appraised as uncontrollable among individuals with low, but not high, cognitive resources, and elevated perceptions of uncontrollability were then associated with an immediate increase in negative affect. 


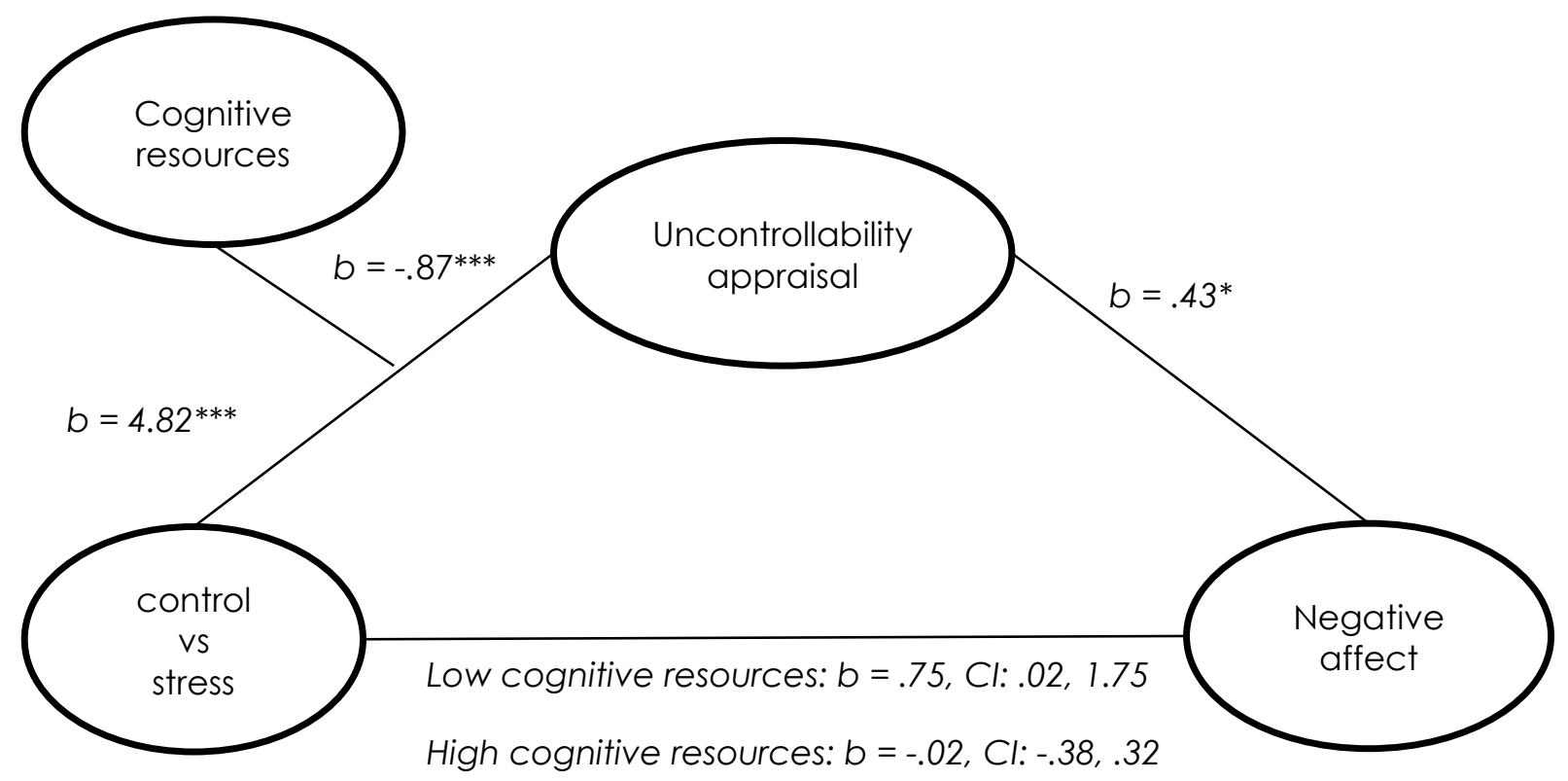

Figure 4. Immediately post-stressor, the mediating role of uncontrollability in the relation between stressor condition and negative affect was moderated by differences cognitive resources. Note: low cognitive resources $=1 S . D$. below the mean, high cognitive resources $=1 S . D$. above the mean. $* * * p<.001, * p<.05$

A somewhat different profile of relations was apparent when measures were taken $40 \mathrm{~min}$ following the stressor. At this time, the relation between stressor condition and negative affect was again mediated by threat appraisal, and this relation was, once more, moderated by individual differences in cognitive control. However, as illustrated in Figure 5, at this time, the relationship between threat appraisal and negative affect was moderated by cognitive control. Specifically, heightened perceived threat was accompanied by the maintenance of elevated negative affect, particularly among individuals with low cognitive control (Figure 5). 


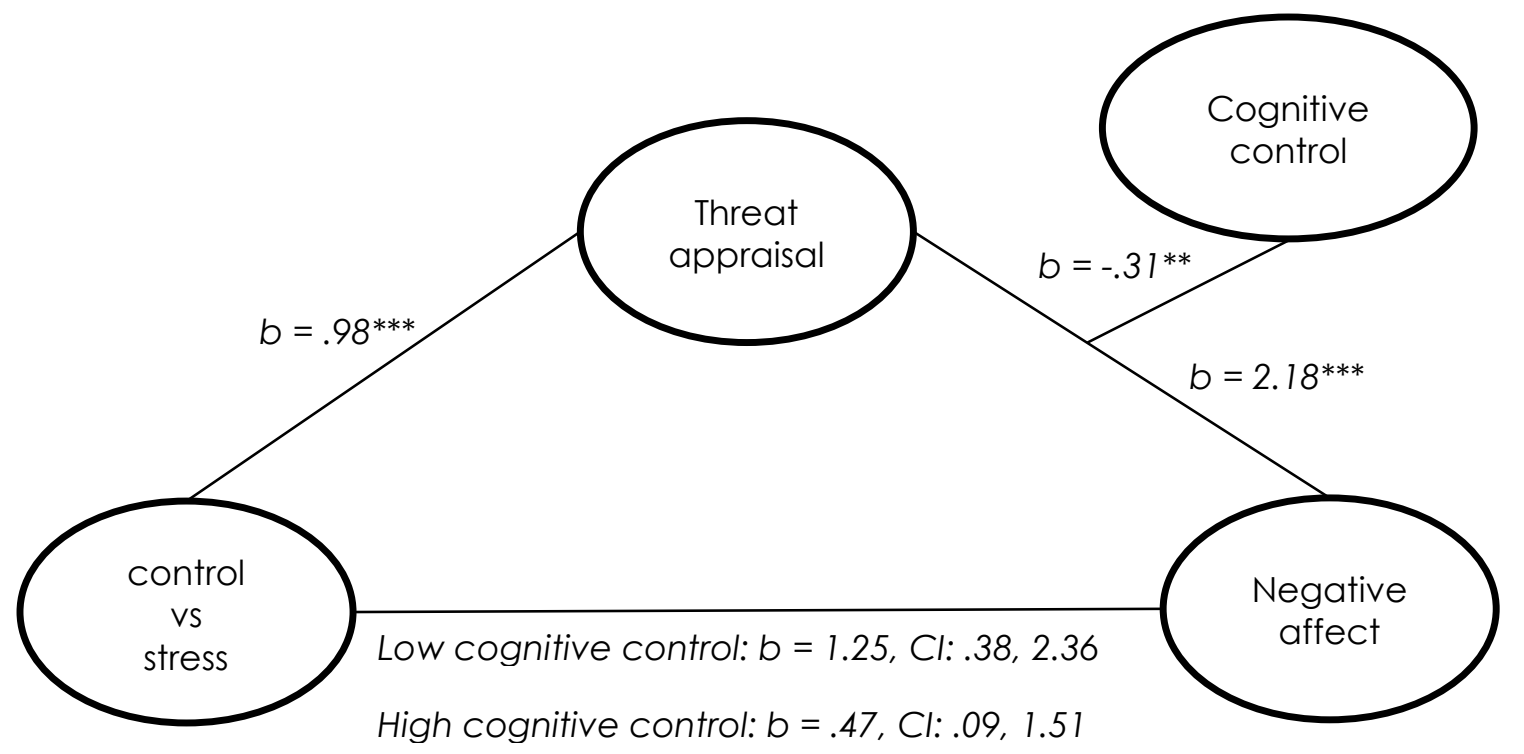

Figure 5. At 40 minutes post-stressor, the mediating role of threat appraisal in the relation between stressor condition and negative affect was moderated by differences cognitive control. Note: low cognitive control $=1 S . D$. below the mean, high cognitive control $=1 S . D$. above the mean. $* * * p<.001, * * p<.01$

The relation between stressor condition and negative affect, which was mediated by threat appraisal, was also moderated by individual differences in cognitive resources. Once again, threat appraisals mediated the relation between stressor condition and negative affect. However, heightened perceived threat at 40 minutes post-stressor was related to sustained negative affect particularly among individuals with low cognitive resources (not shown).

Like the effects observed in Figure 5, the relationship between stressor condition and negative affect 40 minutes following the TSST (control) condition was mediated by perceived uncontrollability. The relation between uncontrollability and negative affect, however, was moderated by differences in cognitive control. Forty minutes following the stressor, as shown in 
Figure 6. the TSST was still appraised as uncontrollable, but perceived uncontrollability was only related to negative affect among individuals with low cognitive control.

This effect was unique to cognitive control as differences in cognitive resources did not moderate the relation between perceived uncontrollability and negative affect. However, an alternative moderated mediation analysis paralleled the findings observed immediately poststressor (see Figure 4). Specifically, the relation between stressor condition and perceptions of uncontrollability was moderated by differences cognitive resources, such that individuals with low cognitive resources still (i.e., 40 minutes after the stressor) appraised the TSST as more uncontrollable than those with high cognitive resources. Greater perceived uncontrollability at this time was then accompanied by the maintenance elevated negative affect (Figure 7). 


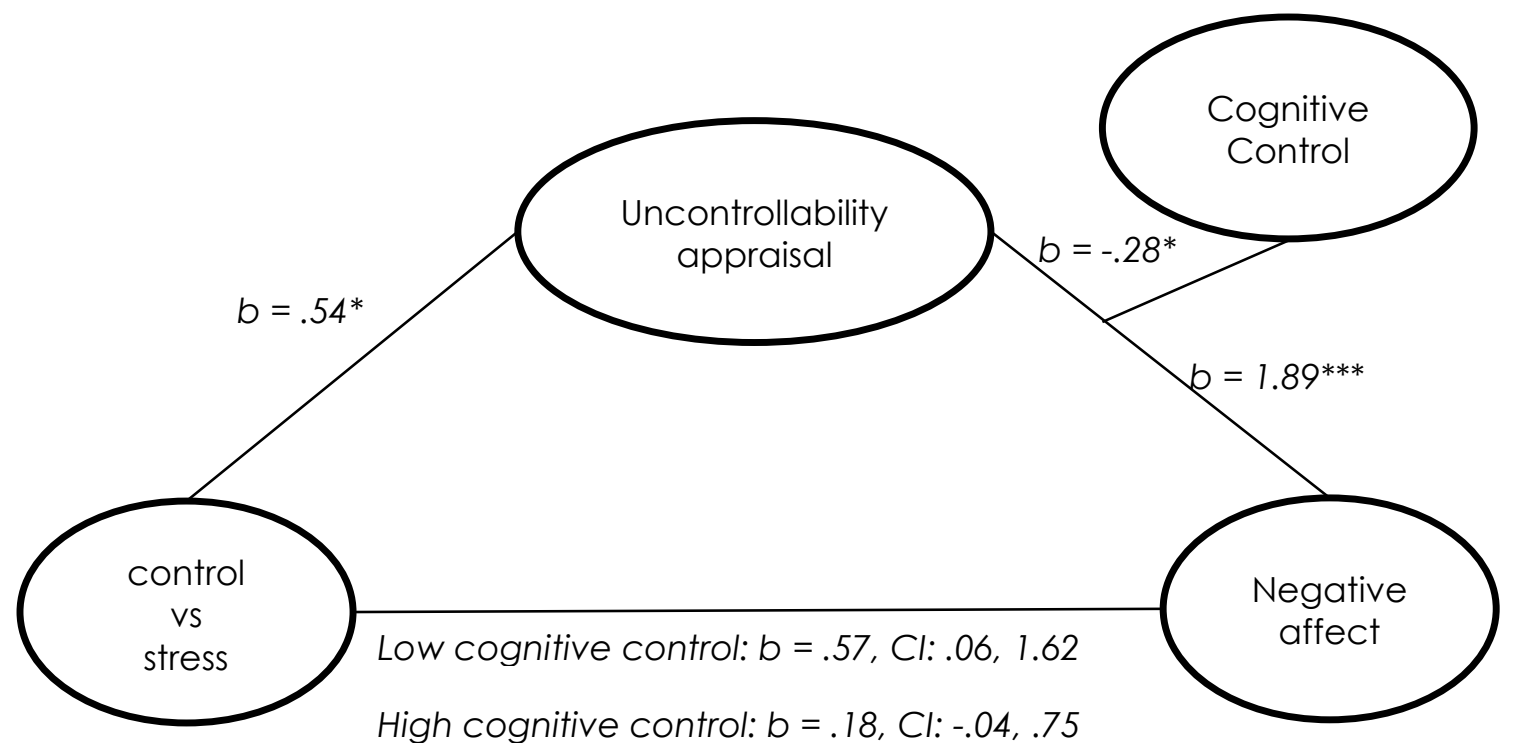

Figure 6. At 40 minutes post-stressor, the mediating role of uncontrollability in the relation between stressor condition and negative affect was moderated by differences cognitive control. Note: low cognitive control $=1 S . D$. below the mean, high cognitive control $=1 S . D$. above the mean. $* * * p<.001, * p<.05$ 


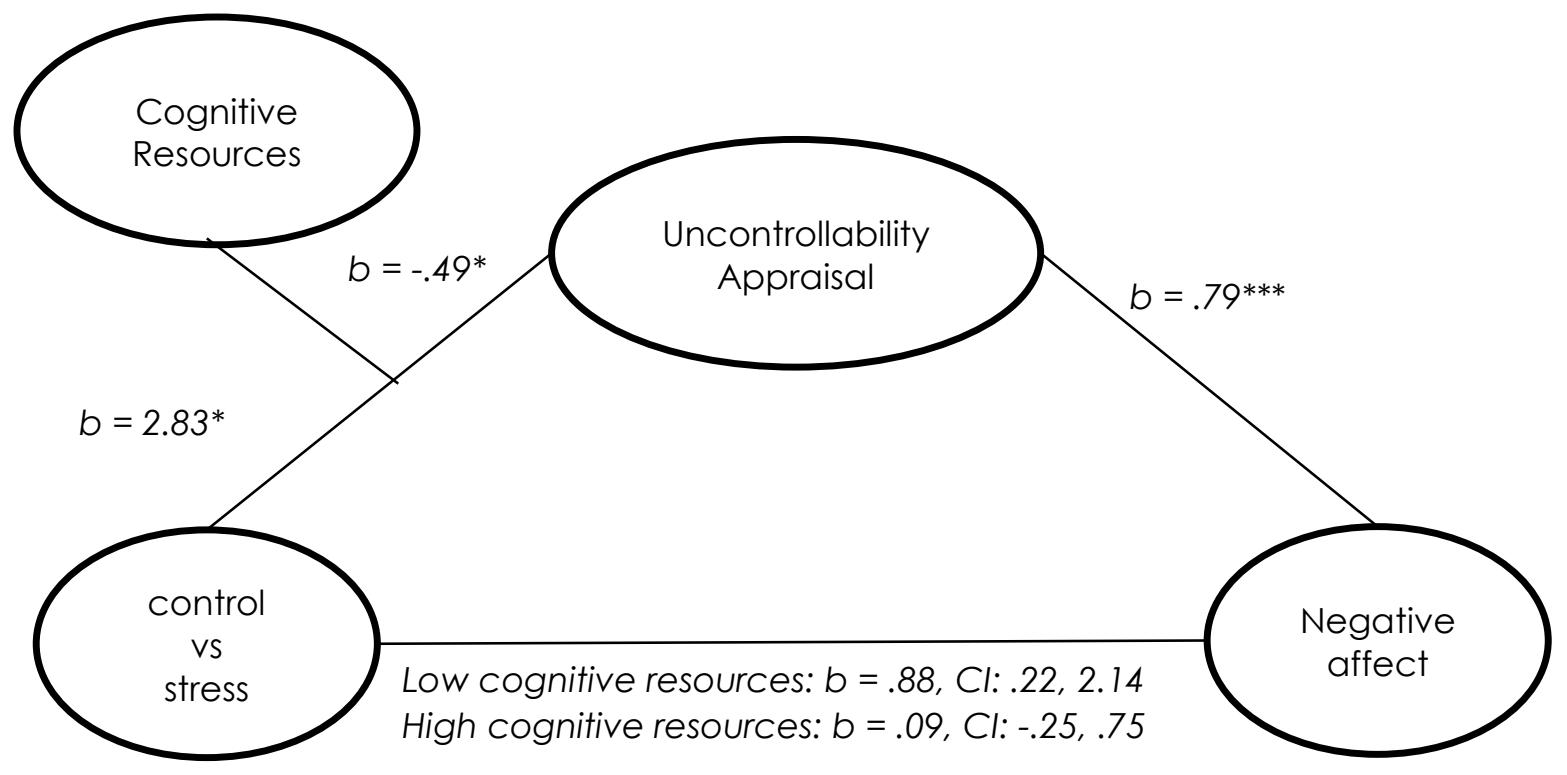

Figure 7. At 40 minutes post-stressor, the mediating role of uncontrollability in the relation between stressor condition and negative affect was moderated by differences cognitive resources. Note: low cognitive resources $=1 S . D$. below the mean, high cognitive resources $=1 S . D$. above the mean. $* * * p<.001, * p<.05$

Plasma cortisol response following the TSST (control) condition

A mixed measures ANOVA of plasma cortisol levels revealed a significant main effect of Time $(F(1,36)=4.34, p=.006)$ as well as a Time $\mathrm{x}$ Condition interaction $F(1,36)=4.67, p=$ .004. Follow-up comparison indicated that individuals in the TSST and control conditions did not differ in plasma cortisol levels at baseline, $t(39)=-.28, p>.05$, or 5 minutes post-stressor, $t$ $(39)=-1.43, p>.05$. By contrast, those in the TSST condition displayed higher plasma cortisol levels 15 minutes, $t(39)=-2.13, p=.04$, and 30 minutes, $t(39)=-2.76, p=.009$, following the TSST, but this difference was less pronounced at 45 minutes post-TSST, $t(42)=-1.77 p=.08$. Consistent with this analysis, an independent samples t-test of cortisol area under the curve, indicated that individuals in the TSST condition $(M=201.71, S . D .=168.74)$ exhibited a 
significantly greater cortisol AUCi relative to those in the control condition $(M=-15.58, S . D .=$ 211.79), $t(39)=-3.62, p<.001$.

The mediating role of stressor appraisals in the relation between stressor condition and plasma cortisol response was moderated by cognitive flexibility

As illustrated in Figure 8, a moderated mediation analysis revealed that the relationship between stressor condition and plasma cortisol AUCi was mediated by heightened perceived uncontrollability, and that this relationship was moderated by differences in cognitive resources. Specifically, the TSST was accompanied by greater perceived uncontrollability among individuals with low, but not high, cognitive resources, and this in turn was associated with elevated plasma cortisol AUCi. In contrast, perceived threat did not mediate the relationship between stressor condition and plasma cortisol AUCi. As well, the relation between stressor condition and plasma cortisol AUCi was not mediated by negative affect. 


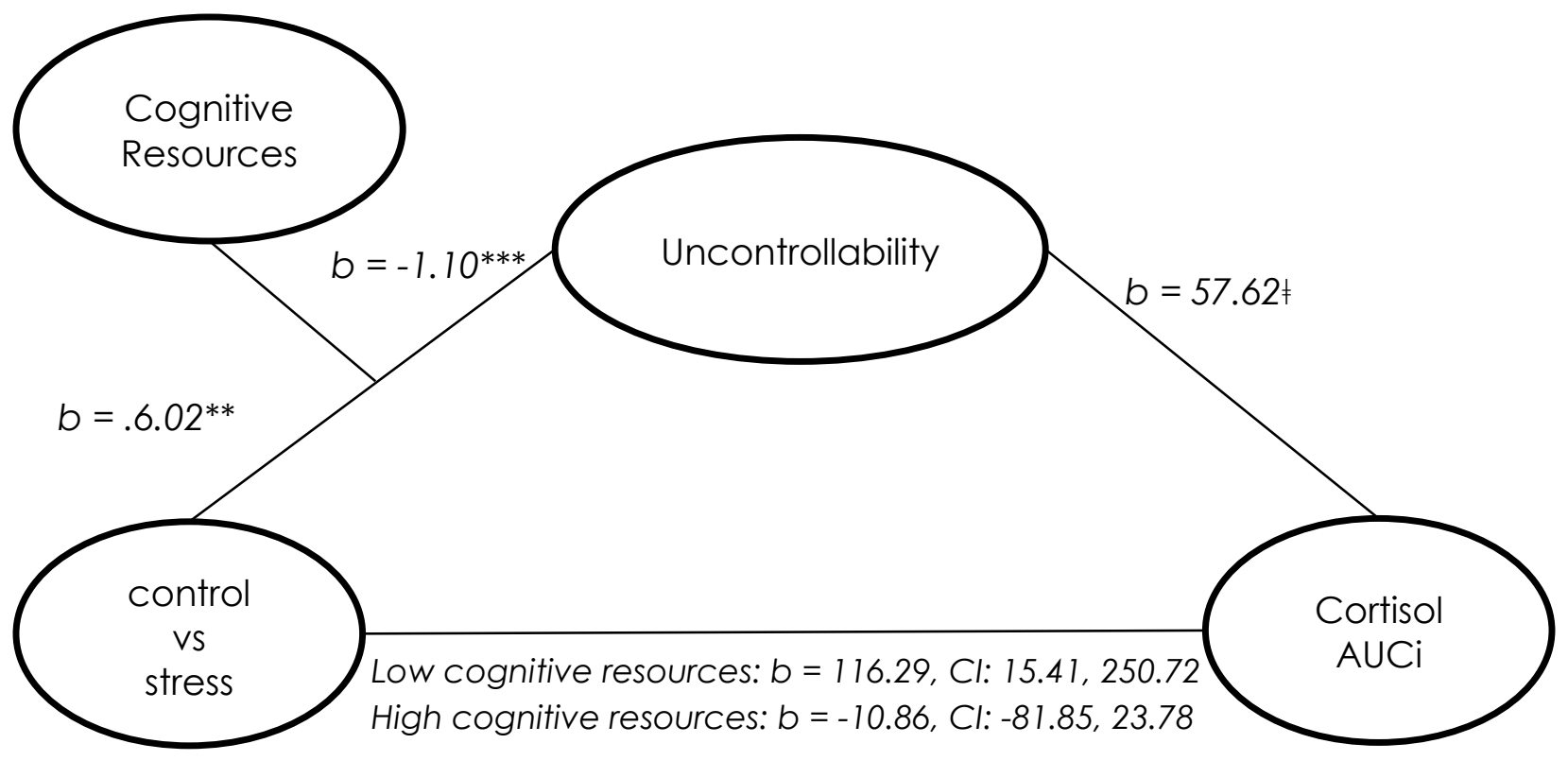

Figure 8. The mediating role of uncontrollability in the relation between stressor condition and plasma cortisol AUCi was moderated by differences in cognitive resources. Note AUCi $=$ Area under the Curve increase; Low cognitive resources $=1$ S.D. below the mean, high cognitive resources $=1$ S.D. above the mean. ${ }^{* * *} p<.001, * * p<.01, \neq p=.09$

\section{DISCUSSION}

Stressful experiences are commonly associated with negative emotional states, yet the intensity and duration of these responses varies considerably between individuals. For some individuals, negative emotional states are relatively transient, whereas for others, negative mood states can persist and become progressively worse over time. Given the reciprocal relationship between cognition and emotion, increased attention has been directed towards understanding how fundamental cognitive control processes, including working memory, response inhibition, and attentional (mental) set-shifting, can serve to regulate emotional responses (Gotlib \& Joormann, 2010; Joormann, 2010; Ochsner \& Gross, 2005; Ochsner et al., 2012). In essence, 
reduced cognitive control has been suggested to result in difficulties disengaging from negative cognitive content (e.g., thoughts or memories), leading to the maintenance and exacerbation of negative mood, and ultimately the emergence of depressive pathology (Joorman et al. 2007; Joorman, 2010). In support of these suggestions, we demonstrated that individual differences in cognitive flexibility, an ability considered to be the manifestation of multiple cognitive control processes, can influence the intensity and duration of negative emotional states following an acute psychosocial stressor.

As expected, in the present study, the TSST was accompanied by an immediate increase in negative affect, although the intensity of this emotional response changed over a relatively brief period. As previously reported (Britton et al., 2012; Hellhammer \& Schubert, 2011), among individuals who experienced the TSST, the intensity of negative affect diminished over the course of 40 minutes, to the point that the TSST and control group no longer differed in negative mood. Positive affect, by contrast, did not differ between the stressor and control groups immediately and 40 minutes following the stressor (control) conditions.

When faced with a stressful situation, the appraisal process plays a central role in the generation of an emotional responses (Lazarus, 2006; Lazarus \& Folkman, 1984). Consistent with this view, immediately post-stressor, the TSST was appraised as more threatening and uncontrollable, and this in turn, was accompanied by an immediate increase in negative affect. However, immediately following the stressor, individuals with low cognitive control appraised the TSST as more threatening than those with high cognitive control, and greater perceived threat was related to increased negative affect. Similarly, individuals with low, but not high, cognitive resources interpreted the TSST as uncontrollable, which in turn, was accompanied by more pronounced negative affect. Indeed, the present findings parallel the evidence 
demonstrating that the utilization of cognitive reappraisal, an emotion regulation strategy that is governed by multiple cognitive control processes, can serve to regulate negative emotional states (Buhle et al., 2014; Goldin et al., 2008; Ochsner \& Gross, 2008)

Interestingly, unlike the observed attenuation in negative affect over time, stressor appraisals appeared to remain relatively stable. At 40 minutes following the stressor, the TSST was still appraised as more threatening and uncontrollable than the control condition. Initially, these findings might seem counterintuitive given the close relationship between appraisals and emotions (Lazarus, 2006), and thus, changes in negative affect would be expected to co-vary with stressor appraisals. Although the appraisal process plays an important role in the initial generation of emotions, the subsequent endorsement of certain coping or emotion regulation strategies can also influence the intensity and duration of an emotional response (Lazarus, 2006; Lazarus \& Folkman, 1984; Nolen-Hoeksema, Wisco, \& Lyubomirsky, 2008). For example, once a stressful situation has been appraised as threatening, which is likely to elicit an initial negative emotional response, the ensuing endorsement of rumination can maintain and exacerbate negative emotions (Nolen-Hoeksema et al., 2008). However, if the initial threat evaluation is followed by cognitive reappraisal (i.e., reinterpreting the meaning of a situation to either reduce negative emotion or increase positive emotions), the initial negative emotional response might dissipate over time (Gross, 2002).

In support of this perspective, we observed that the relationship between stressor condition and negative affect 40 minutes following the stressor encounter was, once again, moderated by individual differences in cognitive control and resources. However, the nature of these moderating effects 40 minutes following the stressor were different than those observed immediately post-stressor. The TSST was still appraised as more threatening and uncontrollable 
than the control condition 40 minutes following the stressor encounter. However, the relationship between heightened threat appraisals and negative affect was most pronounced among individuals with low cognitive control and cognitive resources. Likewise, greater perceived uncontrollability was only related to elevated negative affect among individuals with low, but not high, cognitive control. Moreover, as observed immediately post-stressor, individual differences in cognitive resources moderated the relation between stressor condition and uncontrollability, which in turn, as related to the maintenance of negative affect over time.

In essence, the present findings suggest that individual differences in cognitive flexibility, including cognitive control and resources, can influence the intensity and duration of negative affect following an acute stressor. However, the processes through which this occurs might change over time following a stressful encounter. When first faced with a stressful event, differences in cognitive flexibility might influence how the stressor is initially appraised, which might then impact the immediate emotional response. Over time, however, individual differences in cognitive flexibility might serve to regulate the emotional states that were precipitated by the initial (negative) stressor appraisal.

Together, the present findings are very much in line with the evidence suggesting that disturbances in cognitive control, through influences on emotion regulation, can result in the maintenance and exacerbation of negative emotional states, and increase the risk for dispersive pathology (Joorman, 2010). The present findings are also consistent with the view that the relation between reduced cognitive flexibility (including cognitive control and resources) and elevated depressive symptoms was mediated by unfavorable stressor appraisals as well as the endorsement of ineffective coping strategies. 
In addition to the precipitation of negative mood states, stressors that elicited shame or anger are typically associated with increased levels of cortisol (Dickerson \& Kemeny, 2004 Rapopow et al., 2010). Similar to findings previously reported (Kirschbaum et al., 1993; Kudielka et al., 2007), individuals who had undergone the TSST displayed an increase of plasma cortisol levels, which, over the course of the experiment declined to the levels observed among those who were in the control condition. Moreover, the relationship between stressor condition and the intensity and duration of plasma cortisol responses was mediated by greater perceived uncontrollability. These findings parallel previous observations indicating that uncontrollable stressors, as opposed to those that were more controllable, were particularly effective in producing elevations in cortisol in humans and corticosterone in rodents (Dickerson \& Kemeny, 2004). These findings also suggest that the appraisal process, namely control appraisals, might play an important role in determining the intensity and duration of cortisol changes following an acute stressor in humans (Gaab et al., 2005).

In the present investigation, differences in cognitive resources moderated the relation between stressor condition and plasma cortisol levels through perceptions of uncontrollability. The TSST was appraised as especially uncontrollable by individuals with low, but not high, cognitive resources, and this in turn, was accompanied by a larger and more prolonged plasma cortisol response. Although no previous studies have examined the contribution of cognitive flexibility to cortisol level changes in response to an acute stressor, the present findings parallel the evidence indicating that cognitive reappraisal, which is arguably a manifestation of cognitive flexibility, can influence psychological (e.g., emotions) as well as physiological responses to stressors (Denson et al., 2014; Gaab et al., 2003; Jamieson et al., 2012). The present findings are also in line with the evidence showing that rumination, which has also been linked to reduced 
cognitive flexibility (Davis \& Nolen-Hoeksema, 2000), predicted delayed cortisol recovery following a stressor (Strewart et al., 2013; Zoccola, Quas, \& Yim, 2010; Zoccola, Dickerson, \& Zaldivar, 2008)

\section{Limitations and conclusion}

Several limitations need to be considered when interpreting the findings of the present study. The number of participants in the study was modest, and thus did not allow for analyses of several potentially important moderating variables (e.g., gender). Further, the CFQ, which measured trait-like, as opposed to state, cognitive control and resources. As such, it was not possible to determine whether individuals with high or low cognitive control and resources engaged in the behaviours comprising these constructs (e.g., considered the stressor from multiple viewpoints, reappraised the stressor to reduce negative thoughts and emotions, and shifted their attention away from negative cognitive and emotional states) during and following the stressor encounter. Nevertheless, these findings parallel previous studies demonstrating that stable individual differences which can influence how an individual approaches stressful situations, including trait mindfulness, emotional intelligence, and resilience, can buffer the effects of the TSST on negative emotional states (Brown, Weinsten, \& Creswell, 2012; Mikolajczak et al., 2007; Mikolajczak et al., 2010).

Despite these limitations, the present findings support the growing body of evidence indicating that disturbances in cognitive control processes might contribute to exaggerated and sustained negative emotional and neuroendocrine responses that are typically elicited by stressors. From this perspective, it might be expected that enhancing cognitive control and flexibility might play an important role in promoting effective emotion regulation strategies, which can enhance resilience. 


\section{General Discussion}

According to cognitive theories of depression, certain cognitive styles, such as negative self-schemas (i.e., attitudes and beliefs) pertaining to the self, world, and future, as well as negative attributional/inferential tendencies, can influence risk of a depressive episode, especially in the presence of a negative life event (Abramson et al., 1989; Beck, 1991). These models have provided valuable insight into the cognitive content (e.g., a sense of worthlessness, hopelessness, and helplessness) that characterize depressive illness (Abramson et al., 1989; Beck, 1991), but much less is known regarding the cognitive processes which lead to the maintenance of negative cognitive states, and thus, sustained negative affect. In an attempt to address this question, efforts have been made to examine how disturbances in fundamental cognitive (executive) control processes, including selective attention, working memory, and inhibitory control, might contribute to sustained negative cognitions and emotions (Joormann, 2010; Joormann \& Gotlib, 2010). In this regard, reduced or impaired cognitive control has been associated with the inability to disengage from negative emotional information, potentially through the utilization of ineffective emotion regulation strategies, such as rumination, at the expense of cognitive reappraisal techniques (Joormann, 2010; Joormann \& Gotlib, 2010). In extending this literature, the initial purpose of the present research was to investigate how cognitive flexibility, which is considered to be the expression of multiple cognitive control processes (Dajani \& Uddin, 2015), can be affected by stressful events.

A commonly held view is that when confronted with a stressful situation, "top-down" cognitive control functions (governed mainly by the prefrontal cortex; PFC) can be weakened, and "bottom-up" processes, mediated by the amygdala and basal ganglia, will predominate. The behavioural expression of this shift from top-down to bottom-up processing reflects a tendency 
to engage in more reflexive and emotionally reactive behavioural responses as opposed to more self-regulatory and goal-oriented behavior (Arsten, 2009; Arsten et al., 2015). In support of this view, reduced or impaired PFC-dependent cognitive abilities (e.g., working memory, inhibition, and flexibility) have been observed following various stressor paradigms, whereas processes mediated by limbic regions (e.g., acquisition and consolidation of emotional information) were enhanced (Cahill et al., 2003; Hupbach \& Fieman, 2012; Kuhlmann et al., 2005; Roozendraal et al., 2009; Schoofs et al., 2009; Stauble et al., 2013). However, other studies have painted a more complex picture, indicating that cognitive control processes might not always be impaired following a stressor and that, under certain circumstances, stressors might facilitate these abilities (Parker et al., 2005; Porcelli et al., 2008; Yeun et al., 2009). Consistent with this perspective, we observed (in Study 1) that an acute psychosocial stressor (i.e., the Trier Social Stress Test; TSST) was accompanied by enhanced cognitive flexibility, as reflected through set-shifting ability on the Wisconsin Card Sorting Test (WCST).

The impact of stressful events on cognitive control functioning appears to be highly dependent upon the characteristics of the stressor that has been encountered (Arsten et al., 2009; Sandi, 2013). In the context of acute stressors, more severe and uncontrollable challenges typically provoked impaired cognitive control functioning (e.g., working memory) more readily than stressors of a mild and controllable nature (Arsten et al., 2009; Sandi, 2013). Moreover, in comparison to acute events, chronic stressor can have much more profound impacts on a broad range of cognitive abilities (Dias-Ferreria et al., 2009; Jett et al., 2017; Mika et al., 2012; Mizoguchi et al., 2000). Thus, it is possible that the enhancing effects on set-shifting performance observed in Study 1 might not have been apparent following a more severe or protracted stressor. 
Individuals vary considerably in their behavioral (and emotional) responses to similar stressful situations, which might be related to differences in the way they appraise the characteristics of a stressor (Lazarus \& Folkman, 1984). In fact, for this reason we focused on the stressor appraisal process throughout the present research, and hypothesized that this process might be essential for cognitive flexibility. Consistent with this perspective, in Study 1, we observed that enhanced set-shifting performance following the TSST was mediated by heightened perceived threat. Accordingly, we hypothesized that when confronted with a stressful situation, heightened (perceived) threat might serve to mobilize necessary cognitive (e.g., attention) resources required to adapt to a continuously changing environment, presumably owing to the engagement of prefrontal cortical neurons. We also suggested that upon being confronted by a threat, differences in perceived controllability over the situation might determine whether an individual engages in flexible or rigid behavioral strategies. In this regard, it was observed in Study 1 that perceived uncontrollability did not differ among individuals in the TSST and in the control condition, and might thus account for the stressor provoking enhanced rather than impaired set-shifting performance. Consistent with this view, it has been suggested that stressors of an uncontrollable nature might be more likely to impair PFC-mediated cognitive abilities than those which are controllable (Artsen, 2009).

Depressive disorders have frequently been aligned with impaired cognitive flexibility, including set-shifting ability, which may stem from dysfunction of prefrontal cortical regions (Austin et al., 2001; Hammer \& Ardal, 2009; Trivedi \& Greer, 2014). In Study 1, the enhancing effects of the acute stressor on set shifting ability did not occur among individuals with elevated depressive symptoms. This observation is in line with the suggestion that depressed individuals might need to engage more cognitive (and neuronal) resources in order to function to the level of 
non-depressed individuals, especially in cognitively demanding situations (Harvey et al., 2005; Matsuo et al., 2007; Walter et al., 2007). Placing such high demands on limited cognitive resources might result in their relatively rapid exhaustion of, ultimately diminishing cognitive functioning (Harvey et al., 2005). Following from this perspective, a loss of prefrontal/cognitive resources might explain account for depressed individuals' tendency to engage in reflexive and emotionally reactive coping and emotion regulation strategies at the cost of more effective, goaloriented coping methods, including problem solving and cognitive reappraisal.

Early life adversity, including traumatic life events, is among the strongest risk factors for depressive disorders (Heim \& Nemeroff, 2001). However, the impact of these events on subsequent depressive risk can be modified, for better or for worse, by an individual's genetic disposition (Heim \& Binder, 2012). Several gene variants have been identified as potential risk factors for depression (Bet al., 2008; Caspi et al., 2003; Gatt et al., 2009; Heim et al., 2009), although the processes (cognitive, affective, or biological) through which each polymorphism contributes to depressive illness varies considerably (Hasler et al., 204). In the present research, we focused on the BDNF Val66Met polymorphism since this neurotrophic factor plays an important role in neuroplasticity (Cunha, 2010; Pang \& Lu 2004; Poo, 2001) and was linked to elevated rumination, especially if early life stressors had been encountered (Beevers et al., 2009; Clasen et al., 2012; Gatt et al., 2009). Consistent with this view, the Met allele of this gene polymorphism was previously associated with rumination, especially in the backdrop of early life stressors (Beevers et al., 2009; Clasen et al., 2012; Gatt et al., 2009) . As indicated earlier, this BDNF polymorphism moderated the relation between traumatic life events and cognitive flexibility, in that greater frequency of traumatic events was accompanied by reduced set-shifting performance, but only among individuals carrying the Met allele of this polymorphism. In light 
of these findings, and those previously reported (D’Amore et al., 2013; Sakata et al., 2013), it was suggested that the Met allele of the BDNF gene (or disruptions in BDNF functioning) might specifically disrupt the ability to shift attention away from a previously, but no longer, appropriate behavioral strategy and towards one that is newly effective (i.e., cognitive flexibility). It was observed in the present investigation that the moderating effect of the BDNF genotype on set-shifting ability was dependent on the nature of the trauma experienced as well as the individual's sex. Greater incidence of physical punishment was related to reduced set-shifting ability among males who carried the Met allele, but not among females with this genotype. However, more frequent emotional abuse was associated with decreased set-shifting among female Met carriers, but not male Met carriers. In contrast to these sex differences, greater frequency of general traumas (e.g., personal injury or illness, death of a loved one, and family disturbances) was associated with reduced set-shifting among both males and females who carried the Met allele. Collectively, these findings underscore the importance of considering the influence of gender differences when examining the impact of stressful events on cognitive and affective functioning, even in gene-association studies. Moreover, these data reinforce earlier findings concerning the sex-dependent influence of stressors on cognitive and emotional functioning, including the occurrence of depressive pathology (Hankin, Mermelstien, \& Roesch, 2007; Nolen-Hoeksema, 2001; Rudolph \& Hammen, 1999; Stroud, Salovey, \& Epel, 2002)

Laboratory-based behavioral tasks designed to assess cognitive flexibility, including the WCST, have provided valuable insight into the nature of cognitive deficits exhibited among depressed individuals. To be sure, the use of the WCST has been critical in understanding the effects of an acute stressor on cognitive flexibility (Chapter 1) and the involvement of the BDNF Val66Met gene in this ability (Chapter 2). Yet, the utility of the WCST and other behavioral 
measures can come with several limitations, particularly in clinical settings. The implementation of behavioral paradigms can be time consuming, difficult to score, and repeated use is subject to practice effects. As such, tracking changes in cognitive flexibility among depressed individuals over time and determining the effectiveness of certain treatment strategies might not be feasible in all settings. Behavioral measures might also not capture other the ways in which cognitive flexibility, or disturbances in this ability, is related to depressive disorders (Johnco, Wuthrich, \& Rapee, 2014). For instance, as suggested throughout the present research, a defining characteristic of depressive pathology might by the inability to disengage from negative cognitive and emotional states (Joorman \& Gotlib, 2010). Thus, a second aim of the present research was to develop a brief self-report measure of cognitive flexibility (the CFQ) that mirrored the flexibility assessed through behavioral tasks (e.g., shifting cognitive or attentional sets). In doing so, it was expected that the cognitive inflexibility measured through this test might provide further insight into the cognitive disturbances associated with depression.

As described in Chapter 3, the CFQ comprises two distinct, yet overlapping, dimensions that assess processes (e.g., attention, appraisal, and coping) through which cognitive flexibility might be expressed in stressful situations. The cognitive control dimension of the CFQ addresses the extent to which an individual can control intrusive and repetitive (primarily negative) thoughts and emotions ordinarily elicited by a stressful situation. The second dimension of the CFQ, the cognitive resources dimension, assesses an individual's ability to engage in a set of deliberate (effortful) behaviours that can facilitate an appropriate, or favorable, appraisal of a stressful situation and the flexible selection of a broad range of coping strategies. As demonstrated by the studies in Chapter 3, reduced cognitive flexibility, as measured by the CFQ, was consistently associated with elevated depressive symptoms, and was also related to 
increased preservative thinking and rumination as well as reduced cognitive reappraisal and coping flexibility. Moreover, even after controlling for other measures (e.g., the cognitive flexibility inventory, the perseverative thinking questionnaire, and the emotion regulation questionnaire), the CFQ remained highly related to depressive symptoms, suggesting that this measure assesses processes linked to depression that are not contained within other self-report measures. In this regard, in Chapter 4 we demonstrated diminished cognitive flexibility, assessed by the CFQ, might contribute to sustained negative emotional and neuroendocrine responses following an acute stressor. Collectively, the findings presented in Chapters 3 and 4 suggested that the CFQ might be a promising and easy-to-use tool that can facilitate the understanding of cognitive processes relevant to depressive disorders. Certainly, however, additional research is needed, including the use of clinical samples of depression and a broader set of individuals, in order to determine the validity of the CFQ across multiple demographics and the factors (e.g., interventions) which might promote the processes measured this questionnaire.

\section{Limitations and conclusions}

As outlined in each of the chapters, the findings of the present research should be interpreted cautiously given several limitations which exist across each of the studies. In this regard, the studies included in the present research comprised relatively young and healthy undergraduate students. Given that stressors can have different effects across the life span (Lupien et al., 2009), and that cognitive functioning varies with age (Glisky, 2007), it might be informative to examine these relationships across different demographic factors. As well, the severity of depressive symptoms in the present series of studies was assessed using a self-report questionnaire in a student sample, which might not be relevant to a clinical sample. It would be of obvious value to consider the validity of the CFQ in the context of clinically depressed 
individuals as well as in community samples. Furthermore, some of the studies (e.g., Chapters 1 and 4) included in the present research consisted of relatively small samples. It was, therefore, not uniformly possible to examine potential individual differences (e.g., in relation to sex and age)in the observed relationships. This said, the purpose of Chapters 1 and 4 was to establish the fundamental effects of stressors on cognitive flexibility (Chapter 1) and how this flexibility might serve to regulate emotional and neuroendocrine responses following a stressor (Chapter 4). Thus, the findings in these chapters should be considered preliminary, although intended to inform larger scale projects that include an evaluation of individual differences.

Despite these limitations, the present research generally supported the notion that disturbances in fundamental cognitive control processes and cognitive flexibility might contribute to the maintenance of negative emotional states (and, perhaps, neuroendocrine activation), thereby favoring the evolution of depressive illness (Gotlib \& Joormann, 2010; Joorman \&Gotlib, 2010). It is possible that targeting these cognitive control processes might be a useful preventative treatment strategy for depression. In support of this view, it has been demonstrated that cognitive control training, through computerized working memory tasks and mindfulness-based techniques, can reduce rumination and depressive symptoms as well as prevent the re-occurrence of depressive episodes among remitted individuals (Brunoni et al., 2014; Chambers et al., 2008; Hoorelbeke et al., 2015). Cognitive training exercises might not only serve to enhance cognitive functioning, in general, resulting in greater occupational and social function functioning, but might also improve emotional regulation, much like physical exercise has frequently been proposed to do so (Bherer, Erickson, \& Liu-Ambrose, 2013; Gomez-Pinilla \& Hillman, 2013; Hogan, Mata, \& Carstensen, 2013). Ultimately, the 
development of methods to promote cognitive control as might be a useful tool to promoting positive mental health. 


\section{References}

Abercrombie, H.C., Schaefer, S.M., Larson, C.L., Oakes, T. R., Lindgren, K. A., Holden, J.E. et al., (1998). Metabolic rate in the right amygdala predicts negative affect in depressed patients. Neuroreport, 9, 3301-07.

Abler, B., Erik, S., Herwig, U., \& Walter, H. (2007). Anticipation of aversive stimuli activates extended amygdala in unipolar depression. Journal of Psychiatry Research, 41(6), 511-22.

Abramson, L.Y., Metalski, G.I., \& Alloy, L.B. (1989). Hopelessness depression: a theory-based subtype of depression. Psychological Review, 96(2), 358-72.

Adolphs R, Tranel D, Damasio H \& Damasion AR (1995). Fear and the human amygdala. Journal of Neuroscience, 15(9), 5879-91.

Alexander, J.K., Hillier, A., Smith, R.M., Tivarus, M.E., \& Beversdorf, D.Q. (2007). Betaadrenergic modulation of cognitive flexibility during stress. Journal of Cognitive Neuroscience, 19(3), 468-478.

Alexopoulos, G. S. (2005). Depression in the elderly. The lancet, 365(9475), 1961-1970.

Alloy, L.B., Abramson, L.Y., Whitehouse, W.G., Hogan, M.E., Tashman, NA ... \& Donovan P. (1999) Depressogenic cognitive styles: predictive validity, information processing and personality characteristics, and developmental origins. Behavior Research and Therapy, 37(6), 503-531.

Anand A, Li Y, Wang Y, Wu J, Gao S, Bukhari L et al., (2005). Activity and Connectivity of Brain Mood Regulating Circuit in Depression: A Functional Magnetic Resonance Study. Biological Psychiatry, 57(10), 1079-88. 
Anisman H, Merali Z \& Hayley S (2008). Neurotransmitter, peptide and cytokine processes in relation to depressive disorder: Comorbidity between depression and neurodegenerative disorders. Progress in Neurobiology, 85(1), 1-74.

Aron AR, Robbins TW \& Poldrack RA (2004). Inhibition and right inferior cortex. Trends in Cognitive Science, 8(4), 170-7.

Arnsten AF \& Goldman-Rakic PS (1985). Alpha 2-adrenergic mechanisms in prefrontal cortex associated with cognitive decline in aged nonhuman primates. Science, 230(4731), 1273-76.

Arnsten, A.F.T. \& Goldman-Rakic, P.S. (1998). Noise stress impairs prefrontal cortical cognitive function in monkeys: evidence for a hyperdopaminergic mechanism. Archives in General Psychiatry, 55:362-369.

Arsten AF (2000). Through the looking glass: differential noradenergic modulation of prefrontal cortical function. Neural Plasticity, (1-2), 133-46.

Arsten AF (2009). Stress signalling pathways that impair prefrontal cortex structure and function. Nature Reviews Neuroscience, 10, 410-22.

Arnsten, A.F.T., Raskind, M.A., Taylor, F.B., \& Connor, D.F. (2015). The effects of stress exposure on prefrontal cortex: Translating basic research into successful treatments for post-traumatic stress disorder. Neurobiology of Stress, 1, 89-99.

Austin MP, Mitchell P \& Goodwin GM (2001). Cognitive deficits in depression: possible implications of functional neuropathology. British Journal of Psychiatry, 178, 200-06.

Baddeley, A. (2003). Working memory: looking back and looking forward. Nature Reviews. Neuroscience, 4(10), 829-839 
Baker PM, Thompson JL, Sweeney JA \& Ragozzino E (2011). Differential Effects of 5-HT2A and 5-HT2C Receptor Blockade on Strategy-Switching, Behavioural Brain Research, 219(1), 123-131.

Barceló, F., \& Knight, R. T. (2002). Both random and perseverative errors underlie WCST deficits in prefrontal patients. Neuropsychologia, 40(3), 349-356.

Barch DM, Sheline YI, Csernansky JG \& Snyder AZ (2003). Working Memory and Prefrontal Cortex Dysfunction: Specificity to Schizophrenia Compared with Major Depression. Biological Psychiatry, 53, 376-84.

Bari A, Theobald, DE, Caprioli D, Mar AC, Aidoo-Micah A, Dalley JW \& Robbins TW (2010). Serotonin modulates sensitivity to reward and negative feedback in a probabilistic reversal learning task in rats. Neuropsychopharmacology, 35, $1290-1301$.

Barnett, J. H., Jones, P. B., Robbins, T. W., \& Müller, U. (2007). Effects of the catechol-Omethyltransferase Val158Met polymorphism on executive function: a meta-analysis of the Wisconsin Card Sort Test in schizophrenia and healthy controls. Molecular psychiatry, 12(5), 502-509.

Bath, K. G., \& Lee, F. S. (2006). Variant BDNF (Val66Met) impact on brain structure and function. Cognitive, Affective, \& Behavioral Neuroscience,6(1), 79-85.

Baudin, A., Blot, K., Verney, C., Estevez, L., Santamaria, J., Gressens, P., ... \& Naudon, L. (2012). Maternal deprivation induces deficits in temporal memory and cognitive flexibility and exaggerates synaptic plasticity in the rat medial prefrontal cortex. Neurobiology of learning and memory, 98(3), 207-214.

Baumann BDP, Krell D, Diekmann S, Leschinger A, Stauch R, Wurthman C, Bernstein H-G, Bogerts B (1999) Reduced volume of limbic system-affiliated basal ganglia in mood 
disorders: preliminary data from a post mortem study. J Neuropsychiatry Clin Neurosci $11: 71-78$

Baxter, L. R., Phelps, M. E., Mazziotta, J. C., et al (1985)/ Cerebral metabolic rates for glucose in mood disorders. Studies with positron emission tomography and fluorodeoxyglucose F18. Archives of General Psychiatry, 42, 441- 447.

Beats BC, Sahakian BJ \& Levy R (1996). Cognitive performance in tests sensitive to frontal lobe dysfunction in the elderly depressed. Psychol Med, 26:591-604.

Beck, A.T., Ward, C. H., Mendelson, M., Mock, J., \& Erbaugh, J. (1961). An inventory for measuring depression. Archives of General Psychiatry, 4, 561-571.

Beck, A. T. (1967). Depression: Causes and treatment. Philadelphia: University of Pennsylvania Press.

Beck AT, Rush AJ, Shaw BF, \& Emery G (1979). Cognitive therapy of depression. New York, The Guilford Press.

Beevers, C.G., Strong, D.R., Meyer, B., Pilkonis, P.A., \& Miller, I.R. (2007). Efficiently assessing negative cognition in depression: an item response theory analysis of the Dysfunctional Attitude Scale. Psychological Assessment, 19(2), 199-209.

Bellebaum C, Koch B, Schwarz M \& Daum I (2008). Focal basal ganglia lesions are associated with impairments in reward-based reversal learning. Brain, 131(3), 829-41.

Belski J \& Pluess M (2009). Beyond Diathesis Stress: Differential Susceptibility to Environmental Influences. Psychological Bulletin, 135(6), 885-908.

Benassi, V.A., Sweeney, P.D., \& Dufour, C.L. (1988). Is there a relation between locus of control orientation and depression? Journal of Abnormal Psychology, 97(3), 357-367. 
Bench CJ, Frackowiak RSJ \& Dolan RJ (1995). Changes in regional cerebral blood flow on recovery from depression. Psychological Medicine, 25(2), 247-61.

Berman MG, Peltier S, Nee DE, Kross E, Deldin PJ \& Jonides J (2011). Depression, rumination and the default network. Scan, 6, 548-555.

Berman KF, Doran AR, Pickar D \& Weinberger DR (1993). Is the mechanism of prefrontal hypofunction in depression the same as in schizophrenia? Regional cerebral blood flow during cognitive activation. British Journal of Psychiatry, 162, 183-92.

Birnbaum S, Gobeske KT, Auerbach J, Taylor JR \& Arnsten AF (1999). A role for norepinephrine in stress-induced cognitive deficits: $\alpha$-1-adrenoceptor mediation in the prefrontal cortex. Biological Psychiatry, 46(9), 1266-1274.

Birrell JM \& Brown VJ (2000). Medial frontal cortex mediates perceptual attentional set shifting in the rat. Journal of Neuroscience, 20(11), 4320-4324.

Bissonette GB, Powell EM \& Roesch MR (2013). Neural structures underlying set-shifting: roles of the medial prefrontal cortex and the anterior cingulate cortex. Behavioural Brain Research, 250(1), 91-101.

Bissonette GB, Martins GJ, Franz TM, Harper ES, Schoenbaum G, \& Powell EM (2008). Double dissociation of the effects of medial and orbital prefrontal cortical lesions on attentional and affective shifts in mice. Journal Neuroscience, 28, 11124-30

Block AE, Dhanji H, Thompson-Tardif SF, \& Floresco SB (2007). Thalamic-prefrontal corticalventral striatal circuitry mediates dissociable components of strategy set shifting. Cerebral Cortex, 17, 1625-36. 
Bondi CO, Jett JD \& Morilak DA (2010). Beneficial effects of desipramine on cognitive function of chronically stressed rats are mediated by alpha1-adrenergic receptors in medial prefrontal cortex. Progress in Neuro-Psychopharmacology and Biological Psychiatry 34, 913-923.

Borg J, Henningsson S, Saijo T, Inoue M, Bah J, Westberg L, et al. (2009): Serotonin transporter genotype is associated with cognitive performance but not regional 5-HT1A receptor binding in humans. International Journal of Neuropsychopharmacology, 12, 783-792.

Bosia M, Anselmetti S, Pirovano A, Ermoli E, Marino E, Bramanti P, Smeraldi E \& Cavallaro R (2010). 5HTTLPR functional polymorphism in schizophrenia: executive vs. sustained attention dissociation. Prog. neuropsychopharmacol. Biological Psychiatry, 34(1), 81-85.

Botvinick MM, Cohen JD \& Carter CS (2004). Conflict monitoring and anterior cingulate: an update. Trends in Cognitive Science, 8(12), 539-46.

Borg J, Henningsson S, Saijo T, Inuoe M, Bah J, Westberg L et al., (2009). Serotonin transporter genotype is associated with cognitive performance but not regional 5-HT1A receptor binding in humans. International Journal of Neuropsychopharmacology, 12, 783-792.

Boulougouris V, Glennon JC \& Robbins TW (2008). Dissociable effects of selective 5-HT2A and 5-HT2C receptor antagonists on serial spatial reversal learning in rats. Neuropsychopharmacol, 33(8), 2007-2019.

Boulougouris A, Castane A \& Robbins TW (2009). Dopamine D2/D3 receptor agonist quinpirole impairs spatial reversal learning in rats: investigation of D3 receptor involvement in persistent behavior. Psychopharmacology, 202, 611-20.

Boulougouris V \& Robbins TW (2010). Enhancement of spatial reversal learning by 5-HT2C receptor antagonism is neuroanatomically specific. Journal of Neuroscience, 30, 930-938. 
Bradley, B.P., Mogg, K., Millar,N., \& White,J.(1995a). Selective processing of negative information: effects of clinical anxiety, concurrent depression, and awareness. $J$. Abnorm.Psychol. 104, 532-536.

Bradley, B.P., Mogg,K., \& Williams, R. (1995b).Implicit and explicit memory for emotioncongruent information in clinical depression and anxiety. Behav.Res.Ther. 34, 865-879.

Braver TS, Reynolds JR \& Donaldson DI (2003). Neural mechanisms of transient and sustained cognitive control during task switching. Neuron, 39, 713-726.

Bremner, J. D., Bolus, R., \& Mayer, E. A. (2007). Psychometric Properties of the Early Trauma Inventory-Self Report. The Journal of Nervous and Mental Disease, 195(3), 211-218.

Brigman JL, Mathur P, Harvey-White J, Izquierdo A, Saksida LM, Bussey, et al. (2009)

Pharmacological or genetic inactivation of the serotonin transporter improves reversal learning in mice. Cerebral Cortex, 20(8)-1955-63

Broomfield NM, Davies R, MacMahon K, Ali F \& Cross S (2005). Further evidence of attention bias for negative information in late life depression. Geriatric Psychiatry, 22(2), 175-80.

Brown HD, Osisioma S, Sweeney JA, Ragozzino ME \& Washington DC (2008). The selective serotonin reuptake inhibitor, escitalopram, enhances probabilistic reversal learning in rats. SFN Abstract.

Brown, K. W., Weinstein, N., \& Creswell, J. D. (2012). Trait mindfulness modulates neuroendocrine and affective responses to social evaluative threat. Psychoneuroendocrinology, 37(12), 2037-2041.

Bruder GE, Keilp JG, Xu H, Shikhan M, Gorman JM \& Gilliam TC (2005). Catechol-OMethyltransferase (COMT) Genotypes and Working Memory: Associations with Differing Cognitive Operations. Biological Psychiatry, 58, 901-07. 
Bueller JA, Aftab M, Sen S, Gomez-Hassan D, Burmeister M \& Zubieta JK (2005). BDNF Val66Met Allele Is Associated with Reduced Hippocampal Volume in Healthy Subjects. Biological Psychiatry, 59(9), 812-15.

Buhle, J. T., Silvers, J. A., Wager, T. D., Lopez, R., Onyemekwu, C., Kober, H., ... \& Ochsner, K. N. (2014). Cognitive reappraisal of emotion: a meta-analysis of human neuroimaging studies. Cerebral Cortex, 24(11), 2981-2990.

Burnham KE, Baxter MG, Bainton JR, Southam E, Dawson LA, Bannerman DM, Sharp T (2010). Activation of 5-HT(6) receptors facilitates attentional set shifting.

Psychopharmacology, 208 (1), 13-21.

Butts KA, Floresco SB, \& Phillips AG (2013). Acute stress impairs set-shifting but not reversal learning. Behavior and Brain Research, 252, 222-229.

Byron, K., Khazanchi, S., \& Nazarian, D. (2010). The relationship between stressors and creativity: a meta-analysis examining competing theoretical models. Journal of Applied Psychology, 95(1), 201.

Cahill, L., Gorski, L., \& Le, K. (2003). Enhanced human memory consolidation with postlearning stress: Interaction with the degree of arousal at encoding. Learning \& Memory, 10(4), 270-274.

Cain R, Wasserman MC, Waterhouse BD \& McGaughy JA (2011). Atomoxetine facilitates Attentional Set Shifting in adolescent rats. Developmental Cognitive Neuroscience, 1(4), $552-559$.

Calaminus, C., \& Hauber, W. (2007). Intact discrimination reversal learning but slowed responding to reward-predictive cues after dopamine $\mathrm{d} 1$ and $\mathrm{d} 2$ receptor blockade in the nucleus accumbens of rats. Psychopharmacology, 191(3), 551-566. 
Calaminus, C., \& Hauber, W. (2008). Guidance of instrumental behavior under reversal conditions requires dopamine $\mathrm{d} 1$ and $\mathrm{d} 2$ receptor activation in the orbitofrontal cortex. Neuroscience, 154(4), 1195-1204.

Caldwell, W., McInnis, O. A., McQuaid, R. J., Liu, G., Stead, J. D., Anisman, H., \& Hayley, S. (2013). The role of the Val66Met polymorphism of the brain derived neurotrophic factor gene in coping strategies relevant to depressive symptoms. PloS one, 8(6), e65547.

Canli T \& Lesch KP (2007): Long story short: The serotonin transporter in emotion regulation and social cognition. Nature Neuroscience, 10, 1103-1109.

Carballedo, A., Morris, D., Zill, P., Fahey, C., Reinhold, E., Meisenzahl, E., ... \& Frodl, T. (2013). Brain-derived neurotrophic factor Val66Met polymorphism and early life adversity affect hippocampal volume. American Journal of Medical Genetics Part B: Neuropsychiatric Genetics, 162(2), 183-190.

Carver, C.S., Scheier, M.F. \& Weintraub, J.K. (1989) Assessing coping strategies: a theoretically based approach, J. Pers. Soc. Psychol. 59, 73-81.

Caseras, X., Garner, M., Bradley, B. P., \& Mogg, K. (2007). Biases in visual orienting tonegative and positive scenes in dysphoria: an eye movement study. Journal of Abnormal Psychology, 116(3), 491-497.

Castane A, Theobald DE \& Robbins TW (2010). Selective lesions of the dorsomedial striatum impair serial spatial reversal learning in rats. Behavioral Brain Research, 210, 74-83.

Ceaser, A. E., Goldberg, T. E., Egan, M. F., McMahon, R. P., Weinberger, D. R., \& Gold, J. M. (2008). Set-shifting ability and schizophrenia: a marker of clinical illness or an intermediate phenotype? Biological Psychiatry, 64(9), 782-788. 
Cerqueira JJ, Mailliet F, Almeida OF, Jay TM \& Sousa N (2007). The Prefrontal Cortex as a Key Target of the Maladaptive Response to Stress. Journal of Neuroscience, 27(11), 2781-87/

Chamberlain SR, Muller U, Blackwell AD, Clark L, Robbins TW \& Sahakian BJ (2006).

Neurochemical modulation of response inhibition and probabilistic learning in humans. Science, 311, 861-863.

Chamberlain, S. R., Fineberg, N. A., Blackwell, A. D., Robbins, T. W., \& Sahakian, B. J. (2006). Motor inhibition and cognitive flexibility in obsessive-compulsive disorder and trichotillomania. American Journal of Psychiatry. 163(7), 1282-4

Chamberlain SR \& Robbins TW (2013). Noradrenergic modulation of cognition: therapeutic implications. Journal for Psychopharmacology, 27(8), 694-718.

Chelune, G. J., \& Baer, R. A. (1986). Developmental norms for the Wisconsin Card Sorting test. Journal of clinical and experimental neuropsychology, 8(3), 219-228.

Cheng, C. (2001). Assessing coping flexibility in real-life and laboratory settings: A multimethod approach. Journal of Personality and Social Psychology, 80, 814-833.

Cheng, C. (2003). Cognitive and motivational processes underlying coping flexibility: A dualprocess model. Journal of Personality and Social Psychology, 84, 425-438.

Cheng, C., Lau, H.P., \& Chan, M.P. (2014). Coping flexibility and psychological adjustment to stressful life changes: a meta-analytic review. Psychological Bulletin, 140(6): 1582-607.

Chen J, Lipska BK, Halim N, Ma QD, Matsumoto M, Melhem S et al., (2005). Functional Analysis of Genetic Variation in Catechol-O-Methyltransferase (COMT): Effects on mRNA, Protein, and Enzyme Activity in Postmortem Human Brain. American Journal of human Genetics, 75(5), 807-821. 
Chen ZY, Jing D, Bath KG, Ieraci A, Khan T, Siao CJ et al., (2006). Genetic Variant BDNF (Val66Met) Polymorphism Alters Anxiety-Related Behavior. Science, 314(5796), 140-43.

Chiba S, Numakawa T et al., (2012). Chronic restraint stress causes anxiety- and depression-like behaviors, downregulates glucocorticoid receptor expression, and attenuates glutamate release induced by brain-derived neurotrophic factor in the prefrontal cortex. Progress in Neuro-Psychopharmacology and Biological Psychiatry, 39(1), 112-119.

Chudasama Y, Daniels TE, Gorrin DP, Rhodes SEV, Rudebeck PH \& Murray EA (2012). The role of the anterior cingulate cortex in choices based on reward value and reward contingency. Cerebral Cortex, 23(12), 2884-98.

Clarke HF, Dalley JW, Crofts HS, Robbins TW \& Roberts AC (2004) Cognitive inflexibility after prefrontal serotonin depletion. Science, 304, 878-880.

Clarke HF, Walker SC, Crofts HS, Dalley JW, Robbins TW \& Roberts AC (2005). Prefrontal serotonin depletion affects reversal learning but not attentional set shifting. Journal of Neuroscience, 25, 532-538.

Clarke HF, Walker SC, Dalley JW, Robbins TW, et al (2007). Cognitive inflexibility after prefrontal serotonin depletion is behaviorally and neurochemically specific. Cerebral Cortex, 17, 18-27.

Clarke HF, Hill GJ, Robbins TW \& Roberts AC (2011). Dopamine, but not serotonin, regulates reversal learning in the marmoset caudate nucleus. Journal of Neuroscience, 31, 4290-4297. Clarke HF, Dalley JW, Crofts HS, Robbins TW \& Roberts AC (2004). Cognitive inflexibility after prefrontal serotonin depletion. Science, 304, 878-880. 
Clarke HF, Robbins TW \& Roberts AC (2008). Lesions of the Medial Striatum in Monkeys Produce Perseverative Impairments during Reversal Learning Similar to Those Produced by Lesions of the Orbitofrontal Cortex. The Journal of Neuroscience, 28(43), 10972-10982. Cohen MX \& Ranganath C (2005). Behavioral and neural predictors of upcoming decisions. Cognitive Affective and Behavioral Neuroscience, 5, 117-126.

Cohen MX, Elger CE \& Weber B (2008). Amygdala tractography predicts functional connectivity and learning during feedback-guided decision-making, Neuroimage, 39, 13961407.

Colzato LS, Waszak F, Nieuwenhuis S, Posthuma D \& Hommel B (2010). The flexible mind is associated with the catechol-O-methyltransferase (COMT) Val158Met polymorphism: evidence for a role of dopamine in the control of task-switching. Neuropsychologia, 48(9), 2764-2768.

Compton, R. J., Hofheimer, J., \& Kazinka, R. (2013). Stress regulation and cognitive control: evidence relating cortisol reactivity and neural responses to errors. Cognitive, Affective \& Behavioral Neuroscience, 13(1), 152-163.

Cook SC \& Wellman CL (2004). Chronic stress alters dendritic morphology in rat medial prefrontal cortex. Journal of Neurobiology, 60(2), 236-48.

Cools AR, Van Den Bercken JHL, Horstink MWI, Van Spaendonck KPM, Berger HJC (1984).Cognitive and motor shifting aptitude disorder in Parkinson's disease. Journal of Neurology Neurosurgery and Psychiatry, 47, 443-53.

Cools R, Barker RA, Sahakian BJ \& Robbins TW (2001a). Enhanced or impaired cognitive function in Parkinson's disease as a function of dopaminergic medication and task demands. Cerebral Cortex, 11, 1136-43. 
Cools R, Barker RA, Sahakian BJ \& Robbins TW (2001b). Mechanisms of cognitive set flexibility in Parkinson's disease. Brain, 124: 2503-12.

Cools R, Clark L, Owen AM, \& Robbins TW (2002) Defining the neural mechanisms of probabilistic reversal learning using event-related functional magnetic resonance imaging. Journal of Neuroscience, 22, 4563-4567.

Cools R, Barker RA, Sahakian BJ \& Robbins TW (2003). L-Dopa medication remediates cognitive inflexibility, but increases impulsivity in patients with Parkinson's disease. Neuropsychologia, 41, 1431-41.

Cools R, Clark L \& Robbins TW (2004). Differential responses in human striatum and prefrontal cortex to changes in object and rule relevance. Journal of Neuroscience, 24, 112935.

Cools R, Ivry R \& D’Esposito M (2006). The human striatum is necessary for responding to changes in stimulus relevance. Journal of Cognitive Neuroscience, 18, 1959-72.

Cools R (2006). Dopaminergic modulation of cognitive function-implications for L-DOPA treatment in Parkinson's disease. Neuroscience and Biobehavioral Reviews, 30, 1-23.

Cools R (2008). Role of dopamine in the motivational and cognitive control of behaviour. The Neuroscientist, 14, 381-95.

Cool R \& D'Esposito N (2011). Inverted-U-shaped dopamine actions on human working memory and cognitive control. Biological Psychiatry, 69(12), 133-25.

Cooney R. E., Joormann J., Eugene F., Dennis E. L., Gotlib I. H. (2010). Neural correlates of rumination in depression. Cogn. Affect. Behav. Neurosci. 10, 470-478

Cosgrove, K. P., Mazure, C. M., \& Staley, J. K. (2007). Evolving knowledge of sex differences in brain structure, function, and chemistry. Biological psychiatry, 62(8), 847-855. 
Cragg, L \& Chevalier, N (2012). The processes underlying flexibility in childhood. Quarterly Journal of Experimental Psychology, 65(2), 209-232.

Crayton JW, Joshi I, Gulati A, Aroro RC \& Wolf (1996). Effect of corticosterone on serotonin and catecholamine receptors and uptake sites in rat frontal cortex. Brain Research, 2(29), $260-62$.

Cunha, C., Brambilla, R., \& Thomas, K. L. (2010). A simple role for BDNF in learning and memory?. Frontiers in molecular neuroscience, $3,1$.

D’Amore DE, Tracey BA \& Parikh V (2013). Exogenous BDNF facilitates strategy set-shifting by modulating glutamate dynamics in the dorsal striatum. Neuropharmacology, 75, 312-323.

Damier P, Hirsh EC, Agid Y \& Graybiel AM (1999). The substantia nigra of the human brain II. Patterns of loos of dopamine-containing neurons in Parkinson's disease. Brain, 122, 143748.

Danet M, Lapiz-Bluhm S \& Morilak DA (2010). A cognitive deficit induced in rats by chronic intermittent cold stress is reversed by chronic antidepressant treatment. International Journal of Neuropsychopharmacology, 1-13.

Darvas M \& Palmiter RD (2011). Contributions of striatal dopamine signaling to the modulation of cognitive flexibility. Biological Psychiatry, 69, 704-747.

Davis M, Rainnie D \& Cassell M (1994). Neurotransmission in the rat amygdala related to fear and anxiety. Trends in Neuroscience, 17(5), 208-14.

Davis M (1992). The role of the amygdala in fear and anxiety. Annual Reviews of Neuroscience, $15,353-75$.

Davis M \& Whalen PJ (2001). The amygdala: vigilance and emotion. Molecular Psychiatry, 6(1), 13-34. 
Davis, R.N., \& Nolen-Hoeksema, S. (2000). Cognitive inflexibility among ruminators and nonruminators. Cognitive Therapy \& Research, 24, 699-711.

De Steno DA \& Schmauss CA (2009) Role for dopamine D2 receptors in reversal learning. Neuroscience, 162, 118-127.

Deary, I. J., Corley, J., Gow, A. J., Harris, S. E., Houlihan, L. M., Marioni, R. E., ... \& Starr, J. M. (2009). Age-associated cognitive decline. British medical bulletin, 92(1), 135-152.

Dennis, J.P., \& Wal, J.S.V. (2010). The Cognitive Flexibility Inventory: Instrument development and estimates of reliability and validity. Cognitive Therapy and Research, 34, 241-253.

Denson, T. F., Creswell, J. D., Terides, M. D., \& Blundell, K. (2014). Cognitive reappraisal increases neuroendocrine reactivity to acute social stress and physical pain. Psychoneuroendocrinology, 49, 69-78.

Deveney, C. M., \& Deldin, P. J. (2006). Cognitive flexibility for emotional information in major depressive disorder and non-psychiatric controls. Emotion, 6(3), 429-437.

Dias R, Robbins TW \& Roberts AC (1996a). Dissociation in prefrontal cortex of affective and attentional shifts. Nature, 380, 69-72.

Dias R, Robbins TW \& Roberts AC (1996b) Primate analogue of the Wisconsin Card Sort Test: effects of excitotoxic lesions of the prefrontal cortex in the marmoset. Behavioral Neuroscience, 110, 872- 886.

Dias R, Robbins TW \& Roberts AC (1997). Dissociable Forms of Inhibitory Control within Prefrontal Cortex with an Analog of the Wisconsin Card Sort Test: Restriction to Novel Situations and Independence from "On-Line” Processing. Journal of Neuroscience, 17(23), 9285-9297. 
Dickerson, S. S., \& Kemeny, M. E. (2004). Acute stressors and cortisol responses: a theoretical integration and synthesis of laboratory research. Psychological bulletin, 130(3), 355.

Ditcher GS, Felder JN \& Smoski MJ (2009). Affective context interferes with cognitive control in unipolar depression: An fMRI investigation. Journal of Affective Disorders, 114(1-3), $131-142$.

Ditzen, B., Schmidt, S., Strauss, B., Nater, U. M., Ehlert, U., \& Heinrichs, M. (2008). Adult attachment and social support interact to reduce psychological but not cortisol responses to stress. Journal of psychosomatic research, 64(5), 479-486.

Donaldson C, Lam D \& Mathews A (2007). Rumination and attention in major depression. Behaviour Research and Therapy, 45(11), 2664-2678.

Dove A, Pollmann S, Schubert T, Wiggins CJ \& von Cramon DY (2000). Prefrontal cortex activation in task switching: an event-related fMRI study. Cognition and Brain Research, 9, 103-109.

Downes JJ, Roberts AC, Sahakian BJ, Evenden JL, Morris RG \& Robbins TW (1989). Impaired extra-dimensional shift performance in medicated and unmedicated Parkinson's disease: evidence for a specific attentional dysfunction. Neuropsychologia, 27, 1329-43.

Dias R, Robbins TW \& Roberts AC (1996a). Dissociation in prefrontal cortex of affective and attentional shifts. Nature, 380, 69-72.

Dias R, Robbins TW, Roberts AC (1996b). Primate analogue of the Wisconsin Card Sorting Test: effects of excitotoxic lesions of the prefrontal cortex in the marmoset. Behavioral Neuroscience, 110, 872-86.

Dias R, Robbins TW \& Roberts AC (1997). Dissociable forms of inhibitory control within prefrontal cortex with an analog of the Wisconsin Card Sort Test: restriction to novel 
situations and independence from "on-line" processing. Journal of Neuroscience, 17, 928597.

Dickerson, S. S., \& Kemeny, M. E. (2004). Acute stressors and cortisol responses: a theoretical integration and synthesis of laboratory research. Psychological bulletin, 130(3), 355.

Downes JJ, Roberts AC, Sahakian BJ, Evenden JL, Morris RG \& Robbins TW (1989): Impaired extra-dimensional shift performance in medicated and unmedicated Parkinson's disease:

Evidence for a specific attentional dysfunction. Neuropsychologia, 27, $1329-43$.

Drevets \& Raichle (1992). Neuroanatomical circuits in depression: Implications for treatment mechanisms. Psychopharmacology Bulletin, 28, 261-274

Drevets WC, Price JL, Simpson JR Jr, Todd RD, Reich T, Vannier M et al (1997) Subgenual prefrontal cortex abnormalities in mood disorders. Nature 386:824-827.

Drevets WC (2000). Neuroimaging studies of mood disorders. Biological Psychiatry, 48, 813829.

Drevets WC (2001) Neuroimaging and neuropathological studies of depression: implications for the cognitive-emotional features of mood disorders. Current Opinion in Neurobiology, 11, $240-249$.

Drevets WC, Gadde K, Krishnan KRR (2004) Neuroimaging studies of depression. In: Charney DS, Nestler EJ, Bunney BS (eds) The neurobiological foundation of mental illness, 2nd edn. Oxford University Press, New York.

Drevets WC \& Price JL (2005) Neuroimaging and neuropathological studies of mood disorders. In: Licinio JWM (ed) Biology of depression: from novel insights to therapeutic strategies. Wiley-VCH Verlag GmbH \& Co., Weinheim. 
Drevets WC, Thase ME, Moses-Kolko EL, Price J, Frank E, Kupfer DJ et al (2007) Serotonin1A receptor imaging in recurrent depression: replication and literature review. Nuclear Medicine Biol, 34:865-877

Drevets WC, Price JL \& Furey ML (2008). Brain structural and functional abnormalities in mood disorders: implications for neurocircuitry models of depression. Brain Structure and Function, 213(1-2), 93-118.

Drewe EA (1974). The effect of type and area of brain lesion on Wisconsin card sorting test performance. Cortex, 10, 159-170.

Devinsky O, Morrel MJ \& Vogt BA (1995). Contributions of anterior cingulate cortex to behaviour. Brain 118, 279-306.

Eizenman, M., Yu, L. H., Grupp, L., Eizenman, E., Ellenbogen, M., Gemar, M., et al. (2003). A naturalistic visual scanning approach to assess selective attention in major depressive disorder. Psychiatry Research, 118, 117-128.

Egan MF, Goldberg TE, Kolachana BS, Callicot JH, Mazzanti CM, Straub RE, et al (2001). Effect of COMTval108/158 met genotype on frontal lobe function and risk for schizophrenia. Proclaims of the National Academy of Science, 98, 6917-6922.

Egan NF, Kojima M, Callicott JH, Goldberg TE, Kolachana BS, Bertolino A et al., (2003). The BDNF val66met Polymorphism Affects Activity-Dependent Secretion of BDNF and Human Memory and Hippocampal Function. Cell, 112, 257-269.

Ehring, T., Zetsche, U., Weidacker, K., Wahl, K., Schonfeld, S., \& Ehlers, A. (2011). The Perseverative Thinking Questionnaire (PTQ): validation of a content-independent measure of repetitive negative thinking. Journal of Behavior Therapy and Experimental Psychiatry, 42(2), 225-232. 
Elliot R, Sahakian BJ, McKay AP, Herrod JJ, Robbins TW \& Paykel ES (1996).

Neuropsychological impairments in unipolar depression: the influence of perceived failure on subsequent performance. Psychological Medicine, 26(5), 975-89.

Elzinga, B. M., Molendijk, M. L., Voshaar, R. C. O., Bus, B. A., Prickaerts, J., Spinhoven, P., \& Penninx, B. J. (2011). The impact of childhood abuse and recent stress on serum brainderived neurotrophic factor and the moderating role of BDNF Val66Met. Psychopharmacology, 214(1), 319-328.

Eslinger PJ (1998) Neurological and neuropsychological bases of empathy. European Journal of Neurology, 39, 193-199.

Evers EA, Cools R, Clark L, Van Der Veen FM, Jolles J, et al. (2005). Serotonergic modulation of prefrontal cortex during negative feedback in probabilistic reversal learning. Neuropsychopharmacology, 30, 1138-47.

Evers EA, Van Der Veen FM, van Deursen JA, Schmitt JA, Deutz NE, Jolles J (2006). The effect of acute tryptophan depletion on the BOLD response during performance monitoring and response inhibition in healthy male volunteers. Psychopharmacology, 187, 200-8.

Evers EA, van dV FM, Fekkes D \& Jolles J (2007). Serotonin and cognitive flexibility: neuroimaging studies into the effect of acute tryptophan depletion in healthy volunteers. Current Medicinal Chemistry, 14, 2989-2995.

Fellows LK \& Farah M (2003). Ventromedial frontal cortex mediates affective shifting in humans: evidence from a reversal learning paradigm, Brain, 126, 1830-1837.

Fossati, P., Ergis, A. M., \& Allilaire, J. F. (2001). Executive functioning in unipolar depression: a review. L'Encephale, 28(2), 97-107. 
Fox MT, Barense MD \& Baxter MG (2003) Perceptual attentional set-shifting is impaired in rats with neurotoxic lesions of posterior parietal cortex. Journal of Neuroscience, 23, 676-681.

Floresco SB, Magyar O, Ghods-Sharifi S, Vexelman C \& Tse MT (2006) Multiple dopamine receptor subtypes in the medial prefrontal cortex of the rat regulate set-shifting. Neuropsychopharmacology, 31, 297-309.

Floresco SB \& Magyar O (2006). Mesocortisol dopamine modulation of executive functions: beyond working memory. Psychopharmacology, 188, 567-585.

Foland-Ross LC \& Gotlib IH (2012). Cognitive and neural aspects of information processing in major depressive disorder: an integrative perspective. Frontiers in Psychology, 12(3), 489.

Folkman S \& Lazarus RS (1986). Stress processes and depressive symptomatology. Journal of Abnormal Psychology, 95(2), 107-113.

Fossati P, Amar G, Raoux N, Ergis AM \& Allilaire JF (1999). Executive functioning and verbal memory in young patients with unipolar depression and schizophrenia. Psychiatry Research, 89(3), 171-87.

Fox MD, Snyder AZ, Vincent JL, Corbetta M, Van Essen DC \& Raichle ME (2005). The human brain is intrinsically organized into dynamic, anticorrelated functional networks. Proc Natl Acad Sci U S A, 102, 9673-9678.

Francis DD, Zaharia MD, Shanks N, Anisman H (1995) Stress-induced disturbances in Morris water-maze performance: interstrain variability. Physiology and Behavior, 58, 57-65.

Frank M. J., Moustafa A. A., Haughey H. M., Curran T., Hutchison K. E. (2007). Genetic triple dissociation reveals multiple roles for dopamine in reinforcement learning. Proc. Natl. Acad. Sci., 104, 16311-16316. 
Freedman, S. A., Gluck, N., Tuval-Mashiach, R., Brandes, D., Peri, T., \& Shalev, A. Y. (2002). Gender differences in responses to traumatic events: A prospective study. Journal of Traumatic Stress, 15(5), 407-413.

Fresco, D.M., Williams, N.L., \& Nugent, N.I. (2006). Flexibility and negative affect: examining the associations of explanatory flexibility and coping flexibility to each other and to depression and anxiety. Cognitive Therapy and Research, 30(2), 201-210.

Fresco DM, Rytwinski NK \& Craighead LW (2007). Explanatory Flexibility and Negative Life Events Interact to Predict Depression Symptoms. Journal of Social and Clinical Psychology, $26(5), 595-608$.

Fu CH, Williams SC, Cleare AJ et al., (2004). Attenuation of the Neural Response to Sad Faces in Major Depression by Antidepressant Treatment. Archives in General Psychiatry, 61, 877889.

Furr A, Lapiz-Bluhm MD \& Morilak DA (2011). 5-HT2A receptors in the orbitofrontal cortex facilitate reversal learning and contribute to the beneficial cognitive effects of chronic citalopram treatment in rats. The International Journal of Neuropsychopharmacology, 15, $1295-1305$.

Garnesfki N \& Kraaij V (2006). Cognitive emotion regulation questionnaire - development of a short 18-item version (CERQ-short). Personality and Individual Differences, 41, 1045-1053.

Gaab, J., Blättler, N., Menzi, T., Pabst, B., Stoyer, S., \& Ehlert, U. (2003). Randomized controlled evaluation of the effects of cognitive-behavioral stress management on cortisol responses to acute stress in healthy subjects. Psychoneuroendocrinology, 28(6), 767-779. 
Gatt, J. M., Nemeroff, C. B., Dobson-Stone, C., Paul, R. H., Bryant, R. A., Schofield, P. R., ... \& Williams, L. M. (2009). Interactions between BDNF Val66Met polymorphism and early life stress predict brain and arousal pathways to syndromal depression and anxiety. Molecular psychiatry, 14(7), 681-695.

Gauntlett-Gilbert J, Roberts RC \& Brown VJ (1999). Mechanisms underlying attentional-set shifting in Parkinson's disease. Neuropsychologia, 37, 605-16.

George, M.S., Ketter, T.A., Parekh, P.I., Rosinsky, N., Ring, H.A., Pazzaglia, P.J., Marangell, L.B., Callahan, A.M., Post, R.M., (1997). Blunted left cingulate activation in mood disorder subjects during a response interference task (the Stroop). J. Neuropsychiatry Clin. Neurosci. $9(1), 55-63$.

Ghahremani DG, Monterosso J, Jentsch JD, Bilder RM \& Poldrack RA (2010). Neural components underlying behavioral flexibility in human reversal learning. Cerebral Cortex. $20,1843-1852$.

Goldin, P. R., McRae, K., Ramel, W., \& Gross, J. J. (2008). The neural bases of emotion regulation: reappraisal and suppression of negative emotion. Biological psychiatry, 63(6), $577-586$.

Golightly KL, Lloyd JA, Hobson JE, Gallagher P, Mercer G and Young AH (2001). Acute tryptophan depletion in schizophrenia. Psychological Medicine, 31, 75-84.

Gotlib IH, Krasnoperova E, Yue DL, Joormann J (2004). Attentional biases for negative interpersonal stimuli in clinical depression. J Abnorm Psychol; 113:127-35

Gotlib IH \& Joorman J (2005). Cognition and Depression: Current Status and Future Directions. Annual Reviews in Clinical Psychology, 6, 285-312. 
Gotlib, I.H., \& Joormann, J. (2010). Cognition and depression: current status and future directions. Annual review of clinical psychology, 6, 285.

Ghods-Sharifi S, Haluk DM \& Floresco SB (2008). Differential effects of inactivation of the orbitofrontal cortex on strategy set-shifting and reversal learning. Neurobiology of Learning and Memory, 89, 567-573.

Grant DA \& Berg EA (1948). A behavioral analysis of degree of reinforcement and ease of shifting to new responses in a Weigl-type card-sorting problem. Journal of Experimental Psychology, 38, 404-411.

Grant MM, Thase ME \& Sweeney (2001). Cognitive Disturbance in Outpatient Depressed Younger Adults: Evidence of Modest Impairment. Biological Psychiatry, 50(1), 35-43.

Grattan LM, Bloomer RH, Archambault FX \& Eslinger PJ (1994). Cognitive flexibility and empathy after frontal lobe lesion. Neuropsychiatry, Neuropsychology and Behavioral Neurology, 7(4), 251-259.

Gross, J.J. (2002) Emotion regulation: affective, cognitive, and social consequences. Psychophysiology 39, 281-291.

Gross JJ \& John OP (2003). Individual differences in two emotion regulation processes: implications for affect, relationships, and well-being. Journal of Personality and Social Psychology, 85(2). 348-62.

Gruenewald, Tara L., Margaret E. Kemeny, Najib Aziz, and John L. Fahey (2004). Acute threat to the social self: shame, social self-esteem, and cortisol activity. Psychosomatic medicine, 66 (6), 915-924. 
Gusnard DA, Akbudak E, Shulman GL \& Raichle ME (2001). Medial prefrontal cortex and selfreferential mental activity: relation to a default mode of brain function. Proc. Natl. Acad. Sci. U.S.A. 98, 4259-4264

Haluk DM \& Floresco SB (2009). Ventral striatal dopamine modulation of different forms of behavioral flexibility. Neuropsychocopharmacology, 34(8), 2041-52.

Hamilton JP \& Gotlib IH (2008). Neural substrates of increased memory sensitivity for negative stimuli in major depression. Biological Psychiatry, 63, 1155-1162.

Hamilton JP, Furman DJ, Chang C, Thomason ME, Dennis E \& Gotlib IH (2011). Default-mode and task-positive network activity in major depressive disorder: implications for adaptive and maladaptive rumination. Biological Psychiatry 70, 327-333.

Hampshire A \& Owen AM (2006). Fractionating attentional control using events-related fMRI. Cerebral Cortex, 16, 1679-1689.

Hampshire A, Chaudhry AM, Owen AM \& Roberts AC (2012). Dissociable roles for lateral orbitofrontal cortex and lateral prefrontal cortex during preference driven reversal learning. NeuroImage, 59, 4102-12.

Hankin, B. L., Mermelstein, R., \& Roesch, L. (2007). Sex differences in adolescent depression: Stress exposure and reactivity models. Child development, 78(1), 279-295.

Hariri AR, Goldberg TE, Mattay VS, Kolachana BS, Callicott JH, Egan MF et al., (2003). BrainDerived Neurotrophic Factor val66met Polymorphism Affects Human Memory-Related Hippocampal Activity and Predicts Memory Performance. Journal of Neuroscience, 23(17), $6690-94$. 
Harvey PO, Fossati P, Pochon JB, Levy R, LeBastard G, Lehericy S, et al., (2005). Cognitive control and brain resources in major depression: An fMRI study using the n-back task. Neuroimage, 26, 860-69.

Hasler G, Fromm S, Luckenbaugh DA, Carlson PJ, Waldeck T, Geraci M et al (2008) Neural response to catecholamine depletion in unmedicated, remitted subjects with major depressive disorder and healthy subjects. Arch Gen Psychiatry, 65(5), 521-31.

Hatcher PD, Brown VJ, Tait DS, Bate S, Overend P, Hagan JJ et al (2005). 5-HT6 receptor antagonists improve performance in an attentional set shifting task in rats.

Psychopharmacology, 181, 253-259.

Hayes, A. F. (2013). Introduction to mediation, moderation, and conditional process analysis: A regression-based approach. Guilford Press.

Heaton, R.K., Chelune, G.J., Talley, J.L., Kay, G.C., Curtiss, G. (1993). Wisconsin card sorting test manual. Psychological Assessment Resources, Odessa, FL (1993)

Hirvonen, M., Laakso, A., Nagren, K., Rinne, J.O., Pohjalainen, T. \& Hietala, J. (2004) C957T polymorphism of the dopamine D2 receptor (DRD2) gene affects striatal DRD2 availability in vivo. Mol Psychiatry 9, 1060-1061.

Ho BC, Wassink TH, O’Leary DSO, Sheffield VC \& Anderson NC (2005). Catechol-O-methyl transferase Val158Met gene polymorphism in schizophrenia: working memory, frontal lobe MRI morphology and frontal cerebral blood flow. Molecular Psychiatry, 10, 287-298.

Holmes A \& Wellman CL (2009). Stress-induced prefrontal reorganization and executive dysfunction in rodents. Neuroscience \& Biobehavioural Reviews, 33(6), 773-83. 
Homberg JR, Pattij T, Janssen MC, Ronken E, De Boer SF, Schoffelmeer AN et al (2007). Serotonin transporter deficiency in rats improves inhibitory control but not behavioural flexibility. European Journal of Neuroscience, 26, 2066-2073.

Hornak J, O’Doherty J, Branham J, Rolls ET, Morris RG, Bullock PR \& Polkey CE (2004) Reward-related reversal learning after surgical excisions in orbitofrontal or dorsolateral prefrontal cortex in humans. Journal of Cognitive Neuroscience, 16, 463-478.

Hosang, G. M., Shiles, C., Tansey, K. E., McGuffin, P., \& Uher, R. (2014). Interaction between stress and the BDNF Val66Met polymorphism in depression: a systematic review and metaanalysis. BMC medicine, 12(1), 7.

Hsieh PC, Yeh TL, Lee IH, Huang HC, Chen PS, Yang YK, Chiu NT, Lu RB, Liao MH (2010)

Correlation between errors on the Wisconsin Card Sorting Test and the availability of striatal dopamine transporters in healthy volunteers. Journal of Psychiatry and Neuroscience, 35, 90-94.

Hugdahl K, Rund BR, Lund A, Asbjornsen A, Egeland J, Ersland L, Landro NI, Roness A, Stordal KI, Sundet K \& Thomsen T (2004). Brain activation measured with fMRI during a mental arithmetic task in schizophrenia and major depression. Am. J. Psychiatry 161 (2), 286- 293.

Hughes JH, Gallagher P, Stewart ME, Matthews D, Kelly TP, Young AH (2003) The effects of acute tryptophan depletion on neuropsychological function. Journal of Psychopharmacology, 17, $300-309$.

Hupbach, A. \& Fieman, R. (2012). Moderate Stress Enhances Immediate and Delayed Retrieval of Educationally Relevant Material in Healthy Young Men. Behavioral Neuroscience, 126(6), 819-825. 
Hurtubise J \& Howland JG (2017). Effects of stress on behavioral flexibility in rodents.

Neuroscience, 345, 176-192

Husain MM, McDonald WM, Doraiswamy PM et al (1991) A magnetic resonance imaging study of putamen nuclei in major depression. Psychiatry Res 40:95-99.

Hyafil A, Summerfield C \& Koechlin E (2009). Two mechanisms of task switching in the prefrontal cortex. The Journal of Neuroscience, 29(16), 5135-5142.

Ionesca, T (2012). Exploring the nature of cognitive flexibility. New Ideas in Psychology, 30, 190-200.

Ineichen, C., igrist,H.,Spinelli,S.,Lesch,K.P.,Sautter,E.,Seifritz,E.,etal.(2012). Establishing a probabilistic reversal learning test in mice: evidence for the processes mediating reward-stay and punishment-shift behaviour and for their modulation by serotonin. Neuropharmacology $63,1012-1021$.

Ingram, R.E., Miranda, J., \& Segal, Z. (2006). Cognitive vulnerability to depression. New York: Guilford Press.

Izquierdo A, Wiedholz LM, Millstein RA, Yang RJ, Bussey TJ, Saksida LM et al., (2006).

Genetic and dopaminergic modulation of reversal learning in a touchscreen-based operant procedure for mice. Behavioural Brain Research, 171(2), 181-88.

Izquierdo A, Wellman CL \& Holmes A (2006b). Brief Uncontrollable Stress Causes Dendritic Retraction in Infralimbic Cortex and Resistance to Fear Extinction in Mice. Journal of Neuroscience, 26(21), 5733-38.

Izquierdo A, Newman TK, Higley JD, Murray EA (2007). Genetic modulation of cognitive flexibility and socioemotional behavior in rhesus monkeys. Proclaims of the National Academy of Science, 104, 14128-14133. 
Izquierdo A, Carlos K, Ostrander S, Rodriguez D, McCall-Craddolph A, Yagnik G \& Zhuo F. (2012). Impaired reward learning and intact motivation after serotonin depletion in rats. Behavioural Brain Research, 233, 494-499.

Izquierdo A, Darling C, Manos N, Kim C, Ostrander S, \& Cazares V (2013). Basolateral Amygdala Lesions Facilitate Reward Choices after Negative Feedback in Rats. The Journal of Neuroscience, 33(9), 4105-09.

Jamieson, J. P., Nock, M. K., \& Mendes, W. B. (2012). Mind over matter: reappraising arousal improves cardiovascular and cognitive responses to stress. Journal of Experimental Psychology: General, 141(3), 417.

Jedema HP, Gianaros PJ, Greer PJ, Kerr DD, Liu S, Higley JD et al. (2009) Cognitive impact of genetic variation of the serotonin transporter in primates is associated with differences in brain morphology rather than serotonin neurotransmission. Molecular Psychiatry, 15(5), $512-22$.

Jett JD \& Morilak DA (2013). Too much of a good thing: blocking noradrenergic facilitation in medial prefrontal cortex prevents the detrimental effects of chronic stress on cognition. Neuropsychopharmacology, 38(4), 585-95.

Joober R (2002). Catechol-O-methyltransferase Val-108/158-Met gene variants associated with performance on the Wisconsin card sorting test. Archives in General Psychiatry, 59, 662.

Jocham G, Klein TA, Neumann J, von Cramon DY, Reuter M, Ullsperger M (2009) Dopamine DRD2 polymorphism alters reversal learning and associated neural activity. Journal of Neuroscience, 29, 3695-3704.

Joorman, J. (2004). Attentional bias in dysphoria: The role of inhibitory processes. Cognition \& Emotion, 18, 125-147. 
Joorman J, Yoon L \& Gotlib IH (2007a). Cognitive inhibition in depression. Applied and Preventative Psychology, 12, 128-39.

Joormann, J., Talbot, L., \& Gotlib, I. H. (2007b). Biased processing of emotional information in girls at risk for depression. Journal of Abnormal Psychology, 116, 135- 143.

Joormann, J., \& Gotlib, I. H. (2007). Selective attention to emotional faces following recovery from depression. Journal of Abnormal Psychology, 116, 80-85.

Jorm, A. F., Anstey, K. J., Christensen, H., \& Rodgers, B. (2004). Gender differences in cognitive abilities: The mediating role of health state and health habits. Intelligence, 32(1), 7-23.

Kashdan, T. B., \& Rottenberg, J. (2010). Psychological flexibility as a fundamental aspect of health. Clinical psychology review, 30(7), 865-878.

Kehagia AA, Murray GK \& Robbins TW (2010). Learning and cognitive flexibility: frontostriatal function and monoamenergic modulation. Current Opinion in Neurobiology, 20, 199-204.

Kelly O, Matheson K, Ravindran A, Merali Z \& Anisman H (2007). Ruminative coping among patients with dysthymia before and after pharmacotherapy. Depression \& Anxiety, 24(4), $233-43$.

Kellough JL, Beevers CG, Ellis AJ \& Wells TT (2008). Time course of selective attention in clinically depressed young adults: An eye tracking study. Behaviour Research and Therapy, $1238-1243$.

Kendler, K.S., Karkiwski LM, \& Prescott, CA (1999). Causal relationship between stressful life events and the onset of major depression. The American Journal of Psychiatry, 156(6), $837-841$ 
Kendler, K. S., Thornton, L. M., \& Prescott, C. A. (2001). Gender differences in the rates of exposure to stressful life events and sensitivity to their depressogenic effects. American Journal of Psychiatry, 158(4), 587-593.

Kerns JG, Cohen JD, MacDonald III AW, Cho RY, Stenger VA \& Carter CS (2004). Anterior cingulate conflict monitoring and adjustments in control. Science 303, 1023-1026.

Kessler, R. C., McGonagle, K. A., Swartz, M., Blazer, D. G., \& Nelson, C. B. (1993). Sex and depression in the National Comorbidity Survey I: Lifetime prevalence, chronicity and recurrence. Journal of affective disorders, 29(2-3), 85-96.

Kim C, Johnson NF, Cilles SE \& Gold BT (2011). Common and distinct mechanisms of cognitive flexibility in prefrontal cortex. Journal of Neuroscience, 31, 4771-4779.

Kimberg DY, D’Esposito M \& Martha J (1997). Effects of bromocriptine on human subjects depends on working memory capacity

Kish SJ, Shannak K \& Hornykiewicz O (1988). Uneven pattern of dopamine loss in the striatum of patients with idiopathic Parkinson's disease: pathophysiologic and clinical implications. New England Journal of Medicine, 318, 876-80.

Klanker M, Feenstra M \& Denys D (2013). Dopamenerguc control of cognitive flexibility in humans and animals. Frontiers in Neuroscience, 7, 1-24.

Klein TA, Neumann J, Reuter M, Hennig J, von Cramon DY \& Ullsperger M (2007). Genetically determined differences in learning from errors. Science 318, 1642-1645.

Ko JH, Ptito A, Monchi O, Cho SS, Van ET, Pellechia G et al., (2009). Increased dopamine release in the right anterior cingulate cortex during performance of sorting task: a [11C] FLB 457 PET study. Neuroimage, 46, 516-21. 
Koechlin E \& Hyafil A (2007). Anterior prefrontal function and the limits of human decisionmaking. Science, 318, 594-598.

Konishi S, Nakajima K, Uchida I, Kameyama M, Nakahara K, Sekihara K, Miyashita Y (1998). Transient activation of inferior prefrontal cortex during cognitive set shifting. Nature Neuroscience, 1 (1), 80-84.

Konishi S, Kawazu M, Uchida I, Kikyo H, Asakura I, Miyashita Y (1999a). Contribution of working memory to transient activation in human inferior prefrontal cortex during performance of the Wisconsin Card Sorting Test. Cerebral Cortex, 9, 745-753.

Konishi S, Nakajima K, Uchida I, Kikyo H, Kameyama M, Miyashita Y (1999b) Common inhibitory mechanism in human inferior prefrontal cortex revealed by event-related functional MRI. Brain, 122, 981-991.

Koster, E. H., De Lissnyder, E., Derakshan, N., \& De Raedt, R. (2011). Understanding depressive rumination from a cognitive science perspective: The impaired disengagement hypothesis. Clinical psychology review, 31(1), 138-145.

Kovalchuk, Y., Hanse, E., Kafitz, K. W., \& Konnerth, A. (2002). Postsynaptic induction of BDNF-mediated long-term potentiation. Science, 295(5560), 1729-1734.

Kringelbach ML \& Rolls ET (2004). The functional neuroanatomy of the human orbitofrontal cortex: evidence from neuroimaging and neuropsychology. Progress in Neurobiology, 34172.

Krishnan KRR, McDonald WM, Escalona PR et al (1992) Magnetic resonanace imaging of the caudate nuclei in depression: preliminary observations. Arch Gen Psychiatry 49:553-557. 
Krugel LK, Biele G, Mohr P, Li SC \& Keekeren HR (2009). Genetic variation in dopaminergic neuromodulation influences the ability to rapidly and flexibly adapt decisions. PNAS, 106(42), 17951-56.

Kruzich PJ, GrandyDK (2004) Dopamine D2 receptors mediate two-odor discrimination and reversal learning in C57BL/6 mice. BMC Neuroscience, 5, 12.

Kudielka, B. M., Hellhammer, D. H., Kirschbaum, C., Harmon-Jones, E., \& Winkielman, P. (2007). Ten years of research with the Trier Social Stress Test—revisited. Social neuroscience: Integrating biological and psychological explanations of social behavior, $56-83$.

Kuhlmann, S., Piel, M., \& Wolf, O. T. (2005). Impaired memory retrieval after psychosocial stress in healthy young men. The Journal of Neuroscience, 25(11), 2977-2982.

Kujawa AJ, Torpey D, Kim J, Hajak G, Rose S, Gotlib IH \& Klein DN (2011). Attentional Biases for Emotional Faces in Young Children of Mothers with Chronic or Recurrent Depression. Journal of Abnormal Child Psychology, 39(1), 125-35.

Lane HY, Liu YC, Huang CL, Hsieh CL, Chang YL, Chang L, Chang YC, Chang WH (2008). Prefrontal executive function and $\mathrm{D}(1), \mathrm{D}(3), 5-\mathrm{HT}(2 \mathrm{~A})$ and 5-HT(6) receptor gene variations in healthy adults. Journal of Psychiatry and Neuroscience, 33, 47-53.

Lapiz MD \& Morilak DA (2006). Noradrenergic modulation of cognitive function in rat medial prefrontal cortex as measured by attentional set shifting capability. Neuroscience 137, 10391049.

Lapiz MDS, Bondi CO \& Moralik DA (2007). Chronic Treatment with Desipramine Improves Cognitive Performance of Rats in an Attentional Set-Shifting Test. Neuropsychopharmacology, 32, 1000-1010. 
Lapiz MD, Soto-Pina AE, Hensler JG \& Morilak DA (2009). Chronic intermittent cold stress and serotonin depletion induce deficits of reversal learning in an attentional set-shifting test in rats. Psychopharmacology, 202, 329-341.

Lazarus, R.S. \& Folkman, S. (1984). Stress, appraisal, and coping. New York: Springer.

Lazarus, R. S. (1999). Stress and emotion: A new synthesis. New York: Springer.

Lazarus, R. S. (2006). Stress and emotion: A new synthesis. Springer Publishing Company.

LeDoux JE (2000). Emotion Circuits in the Brain. Annual Review of Neuroscience, 23, 155-84.

Lee B, Groman S, London ED, Jentsch JD (2007): Dopamine D2/D3 receptors play a specific role in the reversal of a learned visual discrimination in monkeys. Neuropsychopharmacology, 32, 2125-2134.

Lie, CH, Spetch, K, Marshall, JC, and Fink, GR (2006). Using fMRI to decompose the neural processes underlying the Wisconsin Card Sorting Test. NeuroImage, 30, 1038-1049.

Liston C, Miller MM, Goldwater DS, Radley JJ, Rocher AB, Hof PR, Morrison JH, McEwen BS (2006). Stress-induced alterations in prefrontal cortical dendritic morphology predict selective impairments in perceptual attentional set-shifting. Journal of Neuroscience, 26, $7870-7874$.

Liston C, McEwen BS \& Casey BJ (2009). Psychosocial stress reversibly disrupts prefrontal processing and attentional control. Proc Natl Acad Sci USA, 106, 912-917.

Logue, S. F., \& Gould, T. J. (2014). The neural and genetic basis of executive function: attention, cognitive flexibility, and response inhibition. Pharmacology Biochemistry and Behavior, 123, 45-54. 
Lovic, V., \& Fleming, A. S. (2004). Artificially-reared female rats show reduced prepulse inhibition and deficits in the attentional set shifting task-reversal of effects with maternal-like licking stimulation. Behavioural brain research, 148(1), 209-219.

Luethi, M., Meier, B., \& Sandi, C. (2008). Stress Effects on Working Memory, Explicit Memory, and Implicit Memory for Neutral and Emotional Stimuli in Healthy Men. Frontiers in Behavioral Neuroscience, 2, 5.

Lupien, S.J., Maheu, F., Tu, M., Fiocco, A., \& Schramek, T.E. (2007). The effects of stress and stress hormones on human cognition: Implications for the field of brain and cognition. Brain and Cognition, 65, 209-237.

Lupien, S. J., McEwen, B. S., Gunnar, M. R., \& Heim, C. (2009). Effects of stress throughout the lifespan on the brain, behaviour and cognition. Nature Reviews Neuroscience, 10(6), 434445.

MacDonald AW, Cohen JD, Stenger VA, Carter CS. 2000. Dissociating the role of dorsolateral prefrontal cortex and anterior cingulate cortex in cognitive control. Science 288:1835-38. Malhotra AK, Kestler LJ, Mazzanti C, Bates JA, Goldberg T, GoldmanD (2002). A functional polymorphism in the COMT gene and performance on a test of prefrontal cognition. American Journal of Psychiatry, 159, 652- 654.

Marin, M. F., Lord, C., Andrews, J., Juster, R. P., Sindi, S., Arsenault-Lapierre, G., ... \& Lupien, S. J. (2011). Chronic stress, cognitive functioning and mental health. Neurobiology of learning and memory, 96(4), 583-595.

Matheson K \& Anisman A (2003). Systems of coping associated with dysphoria, anxiety and depressive illness: a multivariate profile perspective. Stress, 6(3), 223-34. 
Masaki D, Yokoyama C, Kinoshita S, Tsuchida H, Nakatomi Y, Yoshimoto K et al (2006). Relationship between limbic and cortical 5-HT neurotransmission and acquisition and reversal learning in a go/no-go task in rats. Psychopharmacology (Berl) 189: 249-258.

Mathews A \& MacLeod C (2005). Cognitive vulnerability to emotional disorders. Annual Reviews of Clinical Psychology, 1, 167-195.

Mattay VS, Goldberg TE, Fera F, Hariri AR, Tessitore A, Egan MF et al., (2003). Catechol Omethyltransferase val158met genotype and individual variation in the brain response to amphetamine. PNAS, 100(10), 6186-91.

Matsuo K, Glahn DC, Peluso MAM, Hatch JP et al., (2007). Prefrontal hyperactivation during working memory task in untreated individuals with major depressive disorder. Molecular Psychiatry, 12, 158-166.

Mayberg HS, Brannan SK, Mahurin RK, Jerabek PA, Brickman JS, Tekell JL et al (1997) Cingulate function in depression: a potential predictor of treatment response. NeuroReport $8: 1057-1061$

Mayberg, H. S., Liotti, M., Brannan, S. K., McGinnis, S., Mahurin, R. K., Jerabek, P. A., et al. (1999). Reciprocal limbic-cortical function and negative mood: Converging PET findings in depression and normal sadness. American Journal of Psychiatry, 156, 675-682.

Mayberg HS, Lozano AM, Voon V, Mcneely HE, Seminowicz D, Hamani C et al (2005) Deep brain stimulation for treatmentresistant depression. Neuron 45:651-660

Mednick, S. A. (1968). The Remote Associates Test. The Journal of Creative Behavior, 2(3), 213-214.

McAlonan K \& Brown VJ (2003). Orbital prefrontal cortex mediates reversal learning and not attentional set shifting in the rat, Behavioral Brain Research, 146, 97-103. 
McEwen BS (1999). Stress and hippocampal plasticity. Annual Reviews of Neuroscience, 22, $105-22$.

McEwen, B. S., \& Morrison, J. H. (2013). The brain on stress: vulnerability and plasticity of the prefrontal cortex over the life course. Neuron, 79(1), 16-29.

McGaughy J, Ross RS \& Eichenbaum H (2008). Noradrenergic, but not cholinergic, deafferentation of prefrontal cortex impairs attentional set-shifting. Neuroscience, 153(1), $63-71$.

McLean SL, Woolley ML, Thomas D, Neill JC (2009b) Role of 5-HT receptor mechanisms in sub-chronic PCP-induced reversal learning deficits in the rat. Psychopharmacology 206(3), 403-414.

McNab F \& Klingberg T (2007). Prefrontal cortex and basal ganglia control access to working memory. Nature Neuroscience, 103-07.

Mehta MA, Swainson R, Ogilvie AD, Sahakian BJ, Robbins TW (2001) Improved short-term spatial memory but impaired reversal learning following the dopamine D2 agonist bromocriptine in human volunteers. Psychopharmacology, 159, 10-20.

Mehta MA, Manes FF, Magnolfi G, Sahakian BJ \& Robbins TW (2004). Impaired set-shifting and dissociable effects on tests of spatial working memory following the dopamine $\mathrm{D}(2)$ receptor antagonist sulpiride in human volunteers. Psychopharmacology, 176, 331-342.

Meins E, McCarthy-Jones S, Fernyhough C, Lewis G, Bental RP, \& Alloy LB (2012). Assessing negative cognitive style: Developemnt of and validation of a Short-Form version of the Cognitive Style Questionnaire. Personality and Individual Differences, 52(5-2), 581-585. 
Mendelsohn D, Riedel WJ \& Sambeth A (2009). Effects of acute tryptophan depletion on memory, attention and executive functions: a systematic review. Neuroscience and Biobehavioral Reviews, 33, 926-952.

Merriam E, Thase M, Haas G, Keshavan M \& Sweeney JA (1999). Prefrontal cortical dysfunction in depression determined by Wisconsin card sorting test performance. Am J Psychiatry, 156:780-782.

Mikolajczak, M., Roy, E., Luminet, O., Fillée, C., \& de Timary, P. (2007). The moderating impact of emotional intelligence on free cortisol responses to stress.

Psychoneuroendocrinology, 32(8), 1000-1012.

Mikolajczak, M., Roy, E., Luminet, O., \& De Timary, P. (2008). Resilience and hypothalamicpituitary-adrenal axis reactivity under acute stress in young men: Short Communication. Stress, 11(6), 477-482.

Miller EK \& Cohen JD (2001). An integrative theory of prefrontal cortex function. Annual Review of Neuroscience, 24, 167-202.

Milner B (1963). Effects of different brain lesions on card sorting: The role of the frontal lobes. Archives of Neurology, 9, 100-110.

Mittal, C., Griskevicius, V., Simpson, J. A., Sung, S., \& Young, E. S. (2015). Cognitive adaptations to stressful environments: When childhood adversity enhances adult executive function. Journal of personality and social psychology, 109(4), 604.

Miyake A \& Shah P (1999). Toward unified theories of working memory: emerging general consensus, unresolved theoretical issues, and future research directions. In: Miyake, A., Shah, P.(Eds.), Models of Working Memory: Mechanisms of Active Maintenance and Executive Control. Cambridge University Press, Cambridge. 
Monchi O, Petrides M, Petre V, Worsley K \& Dagher A (2001). Wisconsin Card Sorting revisited: distinct neural circuits participating in different stages of the task identified by event-related functional magnetic resonance imaging. Journal of Neuroscience, 21 (19), 7733-7741.

Monchi O, Ko JH \& Strafella AP (2006). Striatal dopamine release during performance of executive functions: a [(11)C] raclopride PET study. Neuroimage, 33, 907-12.

Monsell S (2003). Task Switching. Trends in Cognitive Sciences, 7(3), 134-140.

Monsell S, Sumner P \& Waters H (2003). Task-set reconfiguration with predictable and unpredictable task switches. Memory and Cognition, 31(3), 327-342.

Montague RP, Hyman SE \& Cohen JD (2004). Computational roles for dopamine in behavioural control. Nature, 431, 760-67.

Mueller, S.T. \& Piper, B.J. (2014). The Psychology Experiment Building Language (PEBL) and PEBL Test Battery. Journal of Neuroscience Methods, 222, 250-9.

Murphy FC, Smith KA, Cowen PJ, Robbins TW \& Sahakian BJ (2002). The effects of tryptophan depletion on cognitive and affective processing in healthy volunteers. Psychopharmacology, 163: 42-53.

Murphy FC, Michael A, Robbins TW, Sahakian BJ (2003). Neuropsychological impairment in patients with major depressive disorder: the effects of feedback on task performance. Psychol Med 33: 455-467

Nakahara K, Hayashi T, Konishi S \& Miyashita S (2002). Functional MRI of Macaque Monkeys Performing a Cognitive Set-Shifting Task, Science, 295, 1532-1536. 
Nashiro K, Sakaki M, Nga L \& Mather M (2012). Differential brain activity during emotional versus nonemotional reversal learning. Journal of Cognitive Neuroscience, 24(8), 17941805.

Naegeli KJ, O’Connor JA, Banerjee P \& Morilak DA (2013). Effects of milnacipran on cognitive flexibility following chronic stress in rats. European Journal Pharmacology, 703, 62-66.

Nelson H (1976) A modified card sorting response sensitive to frontal lobe defects. Cortex, 12, 313-324.

Newman LA, Darling J \& McGaughy J (2008). Atomoxitine reverses attentional deficits produced by noradrenergic deafferention of medial prefrontal cortex. Psychopharmacology, 200, 39-50.

Newman LA \& McGaughy J (2011). Attentional effects of lesions to the anterior cingulate cortex: how prior reinforcement influences distractibility. Behavioral Neuroscience, 125(3), $360-71$.

Ng CW, Noblejas MI, Rodefer JS, Smith CB \& Poremba A (2007). Double Dissociation of Attentional Resources: Prefrontal Versus Cingulate Cortices, Journal of Neuroscience, 27(45), 12123-31.

Nikiforuk A \& Popik P (2011). Long-lasting cognitive deficit induced by stress is alleviated by acute administration of antidepressants. Psychopharmacology, 36(1), 28-39.

Nikiforuk A (2012). Dopamine D1 receptor modulation of set-shifting: the role of stress exposure. Behavioural Pharmacology, 23(4), 434-38.

Nolan KA, Bilder RM, Lachman HM, Volavka J (2004) Catechol O-methyltransferase Val158Met polymorphism in schizophrenia: differential effects of Val and Met alleles on cognitive stability and flexibility. American Journal of Psychiatry, 161, 359-361 
Nolen-Hoeksema S (2000). The Role of Rumination in Depressive Disorders and Mixed Anxiety/Depressive Symptoms. Journal of Abnormal Psychology, 109(3), 504-511.

Nolen-Hoeksema, S. (2001). Gender differences in depression. Current directions in psychological science, 10(5), 173-176.

Nolen-Hoeksema S, Wisco BE \& Lyubomirsky S (2008). Rethinking Rumination. Perspectives in Psychological Science, 3(5), 400-24.

O’Doherty J, Kringlebach ML, Rolls ET, Hornak J \& Andrews C (2001). Abstract reward and punishment representations in the human orbital frontal cortex. Nature Neuroscience, 4, 95102.

O’Doherty J, Critchley H, Deichmann R, \& Dolan RJ (2003). Dissociating Valence of Outcome from Behavioral Control in Human Orbital and Ventral Prefrontal Cortices. The Journal of Neuroscience, 23(21), 7931-39.

Ochsner KN \& Gross JJ (2005). The cognitive control of emotion. Trends in Cognitive Science, 9(5), 242-49.

Ochsner, K. N., \& Gross, J. J. (2008). Cognitive emotion regulation: Insights from social cognitive and affective neuroscience. Current directions in psychological science, 17(2), $153-158$.

Ochsner, K. N., Silvers, J. A., \& Buhle, J. T. (2012). Functional imaging studies of emotion regulation: a synthetic review and evolving model of the cognitive control of emotion. Annals of the New York Academy of Sciences, 1251(1), E1-E24.

Okada, G., Okamoto, Y., Morinobu, S., Yamawaki, S., Yokota, N., (2003). Attenuated left prefrontal activation during a verbal fluency task in patients with depression. Neuropsychobiology 47 (1), 21- 26. 
Ortega, L. A., Tracy, B. A., Gould, T. J., \& Parikh, V. (2013). Effects of chronic low-and highdose nicotine on cognitive flexibility in C57BL/6J mice. Behavioural brain research, 238, 134-145.

Owen AM, James M, Leigh PN, Summers BA, Marsden CD, Quinn NP, Lange KW, Robbins TW (1991). Fronto-striatal cognitive deficits at different stages of Parkinson's disease. Brain, 115, 1727-51.

Owen AM, Roberts AC, Hodges JR, Summers BA, Polkey CE, Robbins TW (1993). Contrasting mechanisms of impaired attentional set-shifting in patients with frontal lobe damage or Parkinson's disease. Brain, 116, 1159-75.

Oualian C \& Gisquet-Verrier P (2010). The differential involvement of the prelimbic and infralimbic cortices in response conflict affects behavioral flexibility in rats trained in a new automated strategy-switching task. Learning and Memory, 17, 654-68.

Owen AM, Herrod NJ, Menon DK, Clark JC, Downey SP, Carpenter TA et al., (1999). Redefining the functional organization of working memory processes within human lateral prefrontal cortex. European Journal of Neuroscience, 11(2), 567-74.

Paelecke-Habermann, Y., Pohl, J., \& Leplow, B. (2005). Attention and executive functions in remitted major depression patients. Journal of affective disorders, 89(1), 125-135.

Pang, P.T. \& Lu, B. (2004) Regulation of late-phase LTP and long-term memory in normal and aging hippocampus: role of secreted proteins tPA and BDNF. Ageing Res Rev3, 407-430.

Paradiso, S., Lamberty, G. J., Garvey, M. J., et al (1997) Cognitive impairment in the euthymic phase of chronic unipolar depression. Journal of Nervous and Mental Disease, 185, 748 -754. 
Park SB, Coull JT, McShane RH, Young AH, Sahakian BJ, Robbins TW et al., (1994).

Tryptophan depletion in normal volunteers produces selective impairments in learning and memory. Neuropharmacology, 33, 575-588.

Pattwell, S. S., Bath, K. G., Perez-Castro, R., Lee, F. S., Chao, M. V., \& Ninan, I. (2012). The BDNF Val66Met polymorphism impairs synaptic transmission and plasticity in the infralimbic medial prefrontal cortex. The Journal of Neuroscience, 32(7), 2410-2421.

Parikh, V., Naughton, S. X., Yegla, B., \& Guzman, D. M. (2016). Impact of partial dopamine depletion on cognitive flexibility in BDNF heterozygous mice. Psychopharmacology, 233(8), 1361-1375.

Peacock, E. J., \& Wong, P. T. (1990). The stress appraisal measure (SAM): A multidimensional approach to cognitive appraisal. Stress Medicine, 6(3), 227-236.

Pechtel P \& Pizzagalli DA (2011). Effects of early life stress on cognitive and affectivefunction: an integrated review of human literature. Psychopharmacology, 214-55-70.

Perroud, N., Courtet, P., Vincze, I., Jaussent, I., Jollant, F., Bellivier, F., ... \& Malafosse, A. (2008). Interaction between BDNF Val66Met and childhood trauma on adult's violent suicide attempt. Genes, Brain and Behavior, 7(3), 314-322.

Peterson DA, Elliott C, Song DD, Makeig S, Sejnowski TJ \& Poizner H (2009). Probablistic reversal learning is impaired in Parkinson's disease. Neuroscience, 163(4), 1092-1101.

Petrides M (2005). Lateral prefrontal cortex: architecture and functional organization. Philosophical transactions of the Royal Society of London, 360(1456), 781-95.

Pezawas, L., Verchinski, B. A., Mattay, V. S., Callicott, J. H., Kolachana, B. S., Straub, R. E., ... \& Weinberger, D. R. (2004). The brain-derived neurotrophic factor val66met 
polymorphism and variation in human cortical morphology. The Journal of Neuroscience, 24(45), 10099-10102.

Phelps, E. A., and Anderson, A. K. (1997). Emotional memory: does the amygdala do? Current Opinions in Biology, 7:311-314.

Phillips ML, Drevets WC, Rauch SL \& Lane R (2003). Neurobiology of emotion perception II: implications for major psychiatric disorders. Biological Psychiatry, 54(5), 515-528.

Pizzagalli DA, Iosifescu D, HAllett LA, Ratner KG \& Fava M (2009a). Reduced Hedonic Capacity in Major Depressive Disorder: Evidence from a Probabilistic Reward Task. Journal of Psychiatric Research, 43(1), 76-87.

Pizzagalli DA, Holmes AJ, Dillon DG, Goetz EL, Birk JL, Bogdan R et al (2009b) Reduced caudate and nucleus accumbens response to rewards in unmedicated subjects with major depressive disorder. Am J Psychiatry 166:702-710

Plessow F, FKiesel A \& Kirshbaum C (2011). The stressed prefrontal cortex and goal-directed behaviour: acute psychosocial stress impairs the flexible implementation of task goals. Experimental Brain Research, 216, 397-408.

Poo MM (2001). Neurotrophins as synaptic modulators. Nature Reviews Neuroscience, 2, 2432.

Preacher, K.J., Curran, P.J., and Bauer, D.J. (2006). Computational tools for probing interaction effects in multiple linear regression, multilevel modeling, and latent curve analysis. $J$. Educ. Behav. Stat. 31, 437-448.

Preacher, K.J., Rucker, D.D., Hayes, A.F. (2007). Assessing moderated mediation hypotheses: Theory, methods, and prescriptions. Multivariate Behav. Res. 42, 185-227. 
Preacher, K. J., \& Hayes, A. F. (2008). Asymptotic and resampling strategies for assessing and comparing indirect effects in multiple mediator models. Behavior research methods, 40(3), 879-891.

Pruessner, J. C., Kirschbaum, C., Meinlschmid, G., \& Hellhammer, D. H. (2003). Two formulas for computation of the area under the curve represent measures of total hormone concentration versus time-dependent change. Psychoneuroendocrinology, 28(7), 916931.

Purcell R, Maruff P, Kyrios M, Pantelis C (1997). Neuropsycho-logical function in young patients with unipolar major depression. Psychol Med, 27, 1277-1285

Pzawas L, Verchinski BA, Mattay VS, Callicot JH, Kolachana BS, Straub RE et al., (2004). The Brain-Derived Neurotrophic Factor val66met Polymorphism and Variation in Human Cortical Morphology. Journal of Neuroscience, 24(45), 10099-10102.

Raichle M. E., Macleod A. M., Snyder A. Z., Powers W. J., Gusnard D. A., Shulman G. L. (2001). A default mode of brain function. Proc. Natl. Acad. Sci. U.S.A. 98, 676-682.

Ramos BP, Colgan L, Nou E, Ovadia S, Wilson SR \& Arnsten AF (2005). The Beta-1 Adrenergic Antagonist, Betaxolol, Improves Working Memory Performance in Rats and Monkeys. Biological Psychiatry, 58(11), 894-90.

Ragozzino ME, Detrick S \& Kesner RP (1999). Involvement of the Prelimbic-Infralimbic Areas of the Rodent Prefrontal Cortex in Behavioral Flexibility for Place and Response Learning. Journal of Neuroscience, 19(11), 4585-4594.

Ragozzino ME (2002). The effects of dopamine D1 receptor blockade in the prelimbicinfralimbic areas on behavioural flexibility. Learning and Memory, 9, 18-28. 
Ragozzino ME, Ragozzino KE, Mizumori SJ, Kesner RP (2002). Role of the dorsomedial striatum in behavioral flexibility for response and visual cue discrimination learning. Behavioral Neuroscience, 116, 105-15.

Ragozzina ME, Kim J, Hassert D, et al., (2003). The contribution of the rat prelimbic-infralimbic areas to different forms of task switching. Behavioral Neuroscience, 117, 1054-1065.

Ragozzino ME \& Rozman S (2007). The effect of rate anterior cingulate inactivation on cognitive flexibility, Behavioral Neuroscience, 121 (4), 698-706.

Ramel,W.,Goldin,P.R.,Eyler,L. T.,Brown,G.G.,Gotlib,I.H., and McQuaid,J.R.(2007). Amygdala reactivity and mood-congruent memory in individuals at risk for depressive relapse. Biol.Psychiatry 61, 231-239.

Raspopow, K., Abizaid, A., Matheson, K., \& Anisman, H. (2010). Psychosocial stressor effects on cortisol and ghrelin in emotional and non-emotional eaters: influence of anger and shame. Hormones and Behavior, 58(4), 677-684.

Ravindran, A.V., Anisman, H., Merali, Z., Charbonneau, Y., Telner, J., Bialik, R.J., Wiens, A., Ellis, J. and Griffiths, J. (1999) Treatment of primary dysthymia with cognitive therapy and pharmacotherapy: clinical symptoms and functional impairments, Am. J. Psychiatry 156, $1608-1617$.

Ravindran, A.V., Matheson, K., Griffiths, J., Merali, Z. and Anisman, H. (2002) Stress, coping, uplifts, and quality of life in subtypes of depression: a conceptual model and emerging data, J. Affect. Disord. 71, 121-130.

Ravinilde B, Videbech P, Clemmensen K, Egander A, Rasmussen NA \& Rosenberg R (2002). Cognitive deficits in major depression. Scandinavian Journal of Psychology, 43(3), 239-251. 
Ray R. D., Ochsner K. N., Cooper J. C., Robertson E. R., Gabrieli J. D., Gross J. J. (2005). Individual differences in trait rumination and the neural systems supporting cognitive reappraisal. Cogn. Affect. Behav. Neurosci. 5, 156-168.

Reuter M, Ott U, Vaitl D \& Hennig J (2007). Impaired executive control is associated with a variation in the promoter region of the tryptophan hydroxylase 2 gene. Journal of Cognitive Neuroscience, 19, 401-408.

Ridderinkof, KR, Ullsperger M, Crone EA, Nieuwenhuis S (2004). The role of the medial frontal cortex in cognitive control. Science, 306 (5695), 443-447.

Roberts, A. C., Robbins, T. W., \& Everitt, B. J. (1988). The effects of intradimensional and extradimensional shifts on visual discrimination learning in humans and nonhuman primates. Quarterly Journal of Experimental Psychology, 40B, 321-341.

Robbins TW (2007). Shifting and stopping: front-striatal substrates, neurochemical modulation and clinical implications. Philisophical Transaction of the Royal Society Biological Sciences, 362(1481), 917-32.

Robbins TW \& Arnsten AF (2009). The neuropsychopharmacology of fronto-executive function: monoaminergic modulation. Annual Reviews in Neuroscience, 32, 267-87

Robinson OJ, Cools R \& Sahakian BJ (2012). Tryptophan depletion disinhibits punishment but not reward prediction: implications for resilience. Psychopharmacology, 219, 599-605.

Rodefer JS, Nguyen TN, Karlsson JJ \& Arnt J (2008). Reversal of Subchronic PCP-Induced Deficits in Attentional Set Shifting in Rats by Sertindole and a 5HT6 Receptor Antagonist: Comparison Among Antipsychotics. Neuropsychopharmacology, 33(11), 2657-2666. 
Robinson, M.S., \& Alloy, L.B. (2003). Negative cognitive styles and stress-reactive rumination interact to predict depression; a prospective study. Cognitive Therapy and Research, 27(3), $275-292$.

Rodriguez-Jimenez R, Hoenicka J, Jimenez-Arriero MA, Ponce G, Bagney A, Aragues M et al., (2006). Performance in the Wisconsin Card Sorting Test and the C957T Polymorphism of the DRD2 Gene in Healthy Volunteers. Neuropsychobiology, 54(3), 166-70.

Rogers RD, Blackshaw AJ, Middleton HC, Matthews K, Hawtin K, Crowley C, et al. (1999). Tryptophan depletion impairs stimulus-reward learning while methylphenidate disrupts attentional control in healthy young adults: implications for the monoaminergic basis of impulsive behaviour. Psychopharmacology, 146, 482-491.

Rogers RD, Andrews TC, Grasby PM, Brooks DJ \& Robbins TW (2000). Contrasting cortical and subcortical activations produced by attentional-set shifting and reversal learning in humans. Journal of Cognitive Neuroscience, 12, 142-162.

Rolls ET, Hornack J, Wade D \& McGrath J (1994). Emotion-related learning in patients with social and emotional changes associated with frontal lobe damage. Journal Neurology, Neurosurgery and Psychiatry, 57, 1518-1524.

Rolls, E. T. (1999). The brain and emotion. Oxford: OUP.

Roozendaal, B., McEwen, B.S., \& Chatterji, S. (2009). Stress, memory, and the amygdala. Nature Reviews Neuroscience, 10, 423-433.

Rosa, A., Peralta, V., Cuesta, M.J., Zarzuela, A., Serrano, F., Martinez-Larrea, A. \& Fananas, L. (2004) New evidence of association between COMT gene and prefrontal neurocognitive function in healthy individuals from sibling pairs discordant for psychosis. Am J Psychiatry $161,1110-1112$. 
Rowe JB, Saunders JR, Durantou F \& Robbins TW (1996). Systemic idazoxan impairs performance in a non-reversal shift test: implications for the role of the central noradrenergic systems in selective attention. Journal of Psychopharmacology, 10, 188-194.

Rowe JB, Toni I, Josephs O, Frackowiak RSJ \& Passingham RE (2000) The prefrontal cortex: response selection or maintenance within working memory? Science, 288, 1656 -1660.

Rudolph, K. D., \& Hammen, C. (1999). Age and gender as determinants of stress exposure, generation, and reactions in youngsters: A transactional perspective. Child development, 70(3), 660-677.

Rybakowski JK, Borkowska A, Czerski PM, Skibinski M \& Hauser J (2003). Polymorphism of the brain-derived neurotrophic factor gene and performance on a cognitive prefrontal test in bipolar patients. Bipolar Disorders, 5(6), 468-472.

Rybakowski, J. K., Borkowska, A., Skibinska, M., Szczepankiewicz, A., Kapelski, P., Leszczynska-Rodziewicz, A. ... \& Hauser, J. (2006). Prefrontal cognition in schizophrenia and bipolar illness in relation to Val66Met polymorphism of the brainderived neurotrophic factor gene. Psychiatry and clinical neurosciences, 60(1), 70-76.

Sakata K, Martinowich K, Woo NH, Schloesser RJ, Jimenez DV, Ji Y et al., (2013). Role of activity-dependent BDNF expression in hippocampal-prefrontal cortical regulation of behavioral perseverance. Proclaims of the National Academy of Science, 110(37), 15103-08.

Savitz, J., Solms, M., \& Ramesar, R. (2006). The molecular genetics of cognition: dopamine, COMT and BDNF. Genes, Brain and Behavior, 5(4), 311-328.

Schoofs, D., Wolf, O. T., \& Smeets, T. (2009). Cold pressor stress impairs performance on working memory tasks requiring executive functions in healthy young men. Behavioral Neuroscience, 123, $1066-1075$. 
Schoofs, D., Preuss, D., \& Wolf, O.T. (2008). Psychosocial stress induces working memory impairments in an n-back paradigm. Psychoneuroendocrinology, 33(5), 643-53.

Schultz W, Dayan P \& Montague PR (1997). A neural substrate of prediction and reward. Science, 275(5306), 1593-99.

Shimizu E, Hashimoto K, Olamura N, Koike K, Komatsu N, Kumakiri C, et al (2003). Alterations of serum levels of brain-derived neurotrophic factor (BDNF) in depressed patients with or without antidepressants. Biological Psychiatry, 54, 70-75.

Seamans JK \& Yang CR (2004). The principle features and mechanisms of dopamine in the prefrontal cortex. Progress in Neurobiology, 74(1), 1-58.

Shields, G. S., Kuchenbecker, S. Y., Pressman, S. D., Sumida, K. D., \& Slavich, G. M. (2016). Better cognitive control of emotional information is associated with reduced proinflammatory cytokine reactivity to emotional stress. Stress, 19(1), 63-68.

Siegle GJ, Steinhauer SR, Thase ME, Stenger VA, Carter CS (2002): Can't shake that feeling: fMRI assessment of sustained amygdala activity in response to emotional information in depressed individuals. Biol Psychiatry 51: 693-707.

Siegle GJ, Thompson W, Carter CS, Steinhauer SR \& Thase ME (2007). Increased Amygdala and Decreased Dorsolateral Prefrontal BOLD Responses in Unipolar Depression: Related and Independent Features. Biological Psychiatry, 61(2), 198-209.

Sheline YI, Barch DM, Donnelly JM, Ollinger JM, Snyder AZ \& Mintun MA (2001). Increased amygdala response to masked emotional faces in depressed subjects resolves with antidepressant treatment: an fMRI study. Biology Psychiatry, 50:651-658. 
Smith AG, Neill JC \& Costall B (1999). The dopamine D3/D2 receptor agonist 7-OH-DPAT induces cognitive impairment in the marmoset. Pharmacology Biochemistry and Behavior, $63(2), 201-211$.

Smith AB, Taylor E, Brammer M \& Rubia K (2004). Neural correlates of switching set as measured in fast, event-related functional magnetic imaging. Human Brain Mapping, 21, 247-256.

Smith, S. M., \& Vale, W. W. (2006). The role of the hypothalamic-pituitary-adrenal axis in neuroendocrine responses to stress. Dialogues in Clinical Neuroscience, 8(4), 383-395.

Snyder, H. R. (2013). Major depressive disorder is associated with broad impairments on neuropsychological measures of executive function: a meta-analysis and review. Psychological bulletin, 139(1), 81.

Spann, M. N., Mayes, L. C., Kalmar, J. H., Guiney, J., Womer, F. Y., Pittman, B., ... Blumberg, H. P. (2012). Childhood Abuse and Neglect and Cognitive Flexibility in Adolescents. Child Neuropsychology, 18(2), 182-189.

Spielberger, C. D., Gorsuch, R. L., Lushene, R., Vagg, P. R., \& Jacobs, G. A. (1983). Manual for the State-Trait Anxiety Inventory. Palo Alto, CA: Consulting Psychologists Press.

Sridharan D, Levitin DJ \& Menon V (2008). A critical role for the right fronto-insular cortex in switching between central-executive and default-mode networks. Proc Natl Acad Sci U S A, $105,12569-12574$.

Stalnaker TA, Franz TM, Singh T \& Schoenbaum G (2007). Basolateral amygdala lesions abolish orbitofrontal-dependent reversal impairments. Neuron 54, 51-58. 
Stanton, A. L., Kirk, S. B., Cameron, C. L., \& Danoff-Burg, S. (2000). Coping through emotional approach: Scale construction and validation. Journal of Personality and Social Psychology, 74, 1078-1092.

Stauble, M.R., Thompson, L.A., \& Morgan, G. (2013). Increases in cortisol are positively associated with gains in encoding and maintenance working memory performance in young men. Stress, 16(4), 402-10.

Steinvorth, S, Levine B \& Corkin S (2005). Medial temporal lobe structures are needed to reexperience remote autobiographical memories: evidence from HM and WR. Neuropsychologia, 43, 479-496.

Stefani MR, Groth K, Moghaddam B (2003) Glutamate receptors in the rat medial prefrontal cortex regulate set-shifting ability. Behavioural Neuroscience, 117, 728-737.

Stelzel C, Fiebach CJ, Cools R, Tafazoli S \& D’Esposito M (2013). Dissociable fronto-striatal effects of dopamine D2 receptor stimulation on cognitive versus motor flexibility. Cortex, 49(10), 2799-811.

Stewart, J. G., Mazurka, R., Bond, L., Wynne-Edwards, K. E., \& Harkness, K. L. (2013). Rumination and impaired cortisol recovery following a social stressor in adolescent depression. Journal of abnormal child psychology, 41(7), 1015-1026.

Stroud, L. R., Salovey, P., \& Epel, E. S. (2002). Sex differences in stress responses: social rejection versus achievement stress. Biological psychiatry, 52(4), 318-327.

Stuss, D. T. (1992). Biological and psychological development of executive functions. Brain and cognition, 20(1), 8-23

Stuss DT, Levine B, Alexander MP, Hong J, Palumbo C, Hamer L, et al., (2000). Wisconsin Card Sorting Test performance in patients with focal frontal and posterior brain damage: 
effects of lesion location and test structure on separable cognitive processes.

Neuropsychologia, 38, 388-402.

Sweeney JA, Kmiec JA \& Kupfer DJ (2000). Neuropsychologic impairments in bipolar and unipolar mood disorders on the CANTAB neurocognitive battery. Biological Psychiatry, $48(7), 674-84$

Tait DS, Brown VJ, Farovik A, Theobald DE, Dalley JW \& Robbins TW (2007). Lesions of the dorsal noradrenergic bundle impair attentional set-shifting in the rat. European Journal of Neuroscience, 25, 3719-3724.

Takahashi H, Kato M, Takano H, Arakawa R, Okumura M, Otsuka T et al., (2008). Differential contributions of prefrontal and hippocampal dopamine $\mathrm{D}(1)$ and $\mathrm{D}(2)$ receptors in human cognitive functions. Journal of Neuroscience, 28, 12032-38.

Talbot PS, Watson DR, Barrett SL \& Cooper SJ (2006). Rapid tryptophan depletion improves decision-making cognition in healthy humans without affecting reversal learning or set shifting. Neuropsychopharmacology, 31, 1519-1525.

Taerk E, Grizenko N, Amor LB, Lageix P, Mbekou V, Deguzman R et al., (2004). Catechol-OMethyltransferase (COMT) Val108/158 Met polymorphism does not modulate executive function in children with ADHD. BMC Medical Genetics, 5(30),

Thai CA, Zhang Y \& Howland JG (2013). Effects of acute restraint stress on set-shifting and reversal learning in male rats. Cognitive, Affective, \& Behavioral Neuroscience, 13(1), 164173.

Takao K, Nagatani T, Kitamura Y \& Yamawaki S (1997). Effects of corticosterone on 5-HT1A and 5-HT2 receptor binding and on the receptor-mediated behavioral responses of rats. European Journal of Pharmacology, 333(2-3), 123-28. 
Tavares JV, Clark L, Furey ML, Williams GB, Sahakian BJ \& Drevets WC (2008). Neural basis of abnormal response to negative feedback in unmedicated mood disorders. Neuroimage, 42(3), 1118-1126.

Thomas, A. W., Caporale, N., Wu, C., \& Wilbrecht, L. (2016). Early maternal separation impacts cognitive flexibility at the age of first independence in mice. Developmental cognitive neuroscience, 18, 49-56.

Thorpe SJ, Rolls ET \& Maddison S (1983). Neuronal activity in the orbitofrontal cortex of the behaving monkey. Experimental Brain Research 49, 93-115.

Treynor W., Gonzalez R., Nolen-Hoeksema S. (2003). Rumination reconsidered: a psychometric analysis. Cognit. Ther. Res. 27, 247-259.10.1023

Trivedi, M. H., \& Greer, T. L. (2014). Cognitive dysfunction in unipolar depression: implications for treatment. Journal of affective disorders, 152, 19-27.

Tsai SJ, Younger Y, Chen TJ, Chen TJ, Liou Y, Chen MC et al., (2003). Association study of a functional catechol-O-methyltransferase-gene polymorphism and cognitive function in healthy females. Neuroscience Letters, 338(2), 123-126.

Tunbridge EM, Bannerman DM, Sharp T \& Harrison PJ (2004). Catechol-O-methyltransferase inhibition improves set shifting performance and elevates stimulated dopamine release in the rat prefrontal cortex. Journal of Neuroscience, 24, 5331-35.

Vallender EJ, Lynch L, Novak MA \& Miller GM (2009). Polymorphisms in the 3' UTR of the serotonin transporter are associated with cognitive flexibility in rhesus macaques. American Journal of Medical Genetics Part B: Neuropsychiatric Genetics, 150B, 467-475.

van der Plasse G \& Feenstra MG (2008). Serial reversal learning and acute tryptophan depletion. Behavioral Brain Research, 186, 23-31. 
van Holstein M, Aarts E, van der Schaaf ME, Geurts DE, Verkes RJ, Franke B, et al., (2011). Human cognitive flexibility depends on dopamine D2 receptor signaling. Psychopharmacology, 218, 567-578.

van Laere K, Nuttin B, Gabriels L, Dupont P, Rasmussen S, Greenberg BD et al (2006). Metabolic imaging of anterior capsular stimulation in refractory obsessive-compulsive disorder: a key role for the subgenual anterior cingulate and ventral striatum. J Nucl Med 47:740-747.

Vriezekolk, J.E., van Lankveld, W.G.J.M., Eijsbouts, A.M.M., van Helmond, T., Geenen, R., \& van den Ende, C.H.M. (2012). The coping flexibility questionnaire: development and initial validation in patients with chronic rheumatic diseases. Rheumatology International, 32, 2383-2391.

Walsh CM, Booth V, Poe GR (2011) Spatial and reversal learning in the Morris water maze are largely resistant to six hours of REM sleep deprivation following training. Learning and Memory, 18, 422-34.

Walter H, Wolf RC, Spitzer M \& Vasic N (2007) Increased left prefrontal activation in patients with unipolar depression: an event-related, parametric, performance-controlled fMRI study. $J$ Affect Disord 101:175-185.

Wang X, Zhong P, Gu Z \& Yan Z (2003). Regulation of NMDA receptors by dopamine D4 signaling in prefrontal cortex. Journal of Neuroscience, 23, 9852-9861.

Wang L, Jiao J, Dulawa SC (2011) Infant maternal separation impairs adult cognitive performance in BALB/cJ mice. Psychopharmacology, 216(2), 207-18. 
Watson, D., Clark, L. A., \& Tellegen, A. (1988). Development and validation of brief measures of positive and negative affect: the PANAS scales. Journal of personality and social psychology, 54(6), 1063.

Weiss, E. M., Kemmler, G., Deisenhammer, E. A., Fleischhacker, W. W., \& Delazer, M. (2003). Sex differences in cognitive functions. Personality and individual differences, 35(4), 863875.

Wellman CL (2001). Dendritic Reorganization in Pyramidal Neurons in Medial Prefrontal Cortex after Chronic Corticosterone Administration. Journal of Neurobiology, 29, 245-253.

Whatley, S.L., Foreman, A.C., \& Richards, S. (1998). The relationship of coping style to dysphoria, anxiety, and anger. Psychological Reports, 83(3), 783-791.

Winter S, Dieckmann M \& Schwabe K (2009). Dopamine in the prefrontal cortex regulates rat behavioural flexibility to changing reward value. Behavioural Brain Research, 198, 206213.

Xue X, Shoa S, Wang W \& Shoa F (2013). Maternal Separation Induces Alterations in Reversal Learning and Brain-Derived Neurotrophic Factor Expression in Adult Rats. Neuropsychobiology, 68(4), 243-49.

Yiend, J. (2010). The effects of emotion on attention: a review of attentional processing of emotional information. Cognition and Emotion, 24(1), 3-47.

Yu H \& Chen ZY (2011). The role of BDNF in depression on the basis of its location in the neural circuitry. Acta Pharmacol Sin 32:3-11.

Zeni CP, Trmontina S, Znie TA, Coelho R, Pheula G, Bernardi J et al., (2013) The Val66Met Polymorphism at the BDNF Gene does not Influence Wisconsin Card Sorting Test Results 
in Children and Adolescents with Bipolar Disorder. Revista Brasileira de Psiquiatria, 35(1), 44-50.

Zoccola, P. M., Quas, J. A., \& Yim, I. S. (2010). Salivary cortisol responses to a psychosocial laboratory stressor and later verbal recall of the stressor: The role of trait and state rumination. Stress, 13(5), 435-443.

Zwick, W.R., \& Velicer, W.F. (1986). Comparison of five rules for determining the number of components to retain. Psychological Bulletin, 99(3), 432-442. 


\section{Appendix A: Measures}

\section{Cognitive Flexibility Questionnaire - 26 item (CFQ-26)}

The purpose of this questionnaire is to determine what individuals generally think, feel, and do when experiencing stressful situations. Of course, you may respond differently depending on the situation, but think of what you usually do when you are under a lot of stress. Using the scale below, indicate the extent to which agree or disagree with the following statements. Please answer according to what you actually think/feel/do rather than what you think is the correct response.

\begin{tabular}{ccccccc}
$\begin{array}{c}\text { Strongly } \\
\text { Disagree }\end{array}$ & Disagree & $\begin{array}{c}\text { Slightly } \\
\text { Disagree }\end{array}$ & Neutral & $\begin{array}{c}\text { Slightly } \\
\text { Agree }\end{array}$ & Agree & $\begin{array}{c}\text { Strongly } \\
\text { Agree }\end{array}$ \\
\hline 1 & 2 & 3 & 4 & 5 & 6 & 7
\end{tabular}

\section{Generally, in stressful situations...}

\begin{tabular}{|c|c|c|c|c|c|c|c|c|}
\hline & $\begin{array}{l}\text { I weigh many options before choosing how to } \\
\text { take action. }\end{array}$ & 1 & 2 & 3 & 4 & 5 & 6 & 7 \\
\hline 2. & $\begin{array}{l}\text { I feel like I lose control over my thoughts and } \\
\text { emotions. }\end{array}$ & 1 & 2 & 3 & 4 & 5 & 6 & 7 \\
\hline 3. & $\begin{array}{l}\text { Putting a positive spin on a bad experience } \\
\text { comes fairly easy to me. }\end{array}$ & 1 & 2 & 3 & 4 & 5 & 6 & 7 \\
\hline
\end{tabular}

$\begin{array}{lllllllll}\text { 4. I approach the situation from multiple angles. } & 1 & 2 & 3 & 4 & 5 & 6 & 7 \\ \text { 5. It's difficult for me to let go of intrusive } & 1 & 2 & 3 & 4 & 5 & 6 & 7\end{array}$
thoughts or emotions.

6. I consider the situation for multiple $\quad \begin{array}{llllllll} & 1 & 2 & 3 & 4 & 5 & 6 & 7\end{array}$ viewpoints before responding.

7. I take the time to think of several ways to best $\quad \begin{array}{lllllllll}1 & 2 & 3 & 4 & 5 & 6 & 7\end{array}$ cope with the situation before acting.
8. I find it easy to set-aside unpleasant thought $\quad \begin{array}{lllllllll}1 & 2 & 3 & 4 & 5 & 6 & 7\end{array}$ or emotions.

9. It is easy for me to ignore distracting $\quad \begin{array}{llllllll}1 & 2 & 3 & 4 & 5 & 6 & 7\end{array}$ thoughts.
10. I can easily think of multiple coping options $\begin{array}{lllllll}1 & 2 & 3 & 4 & 5 & 6 & 7\end{array}$ before deciding how to respond.

11. I take the time to see things from different perspectives before reacting. 
12. I get easily distracted by upsetting thoughts or

$\begin{array}{lllllll}1 & 2 & 3 & 4 & 5 & 6 & 7\end{array}$

feelings.

13. It's hard for me to ignore negative emotions $\quad \begin{array}{lllllllll}1 & 2 & 3 & 4 & 5 & 6 & 7\end{array}$ once they have been provoked.
14. I take the time to think of more than one way $\quad \begin{array}{lllllllll}1 & 2 & 3 & 4 & 5 & 6 & 7\end{array}$ to resolve the problem
15. I control my thoughts and feelings by putting $\quad \begin{array}{lllllllll}1 & 2 & 3 & 4 & 5 & 6 & 7\end{array}$ the situation in context.

\begin{tabular}{|c|c|c|c|c|c|c|c|}
\hline $\begin{array}{l}\text { 16. I can remain in control over my thoughts and } \\
\text { emotions. }\end{array}$ & 1 & 2 & 3 & 4 & 5 & 6 & 7 \\
\hline 17. I can easily suppress upsetting memories. & 1 & 2 & 3 & 4 & 5 & 6 & 7 \\
\hline $\begin{array}{l}\text { 18. It is easy for me to shift my attention to other } \\
\text { things if I am upset. }\end{array}$ & 1 & 2 & 3 & 4 & 5 & 6 & 7 \\
\hline $\begin{array}{l}\text { 19. I find it easy to look for something positive, } \\
\text { even when I am stressed. }\end{array}$ & 1 & 2 & 3 & 4 & 5 & 6 & 7 \\
\hline
\end{tabular}

$\begin{array}{llllllll}\text { 20. I have a hard time managing my emotions. } & 1 & 2 & 3 & 4 & 5 & 6 & 7 \\ \text { 21. I control negative thoughts and emotions by } & 1 & 2 & 3 & 4 & 5 & 6 & 7\end{array}$
modifying the way I think about the situation.

22. My thoughts and emotions interfere with my $\quad \begin{array}{lllllllll}1 & 2 & 3 & 4 & 5 & 6 & 7\end{array}$ ability to concentrate.

23. It is easy for me to reassess a negative $\quad \begin{array}{lllllllll} & 1 & 2 & 3 & 4 & 5 & 6 & 7\end{array}$ experience into a positive one.

$\begin{aligned} & \text { 24. I manage my thoughts or feelings by } \\ & \text { reframing the situation. }\end{aligned}$
$\begin{aligned} & \text { 25. It's hard for me to shift my attention away } \\ & \text { from negative thoughts or feelings. }\end{aligned}$
$\begin{aligned} & \text { 26. It's hard for me to put things in perspective } \\ & \text { when I'm upset. }\end{aligned}$




\section{Cognitive Flexibility Questionnaire - 44 item (CFQ-44)}

The purpose of this questionnaire is to determine what individuals generally think, feel, and do when experiencing stressful situations. Of course, you may respond differently depending on the situation, but think of what you usually do when you are under a lot of stress. Using the scale below, indicate the extent to which agree or disagree with the following statements. Please answer according to what you actually think/feel/do rather than what you think is the correct response.

\begin{tabular}{ccccccc}
$\begin{array}{c}\text { Strongly } \\
\text { Disagree }\end{array}$ & Disagree & $\begin{array}{c}\text { Slightly } \\
\text { Disagree }\end{array}$ & Neutral & $\begin{array}{c}\text { Slightly } \\
\text { Agree }\end{array}$ & Agree & $\begin{array}{c}\text { Strongly } \\
\text { Agree }\end{array}$ \\
\hline 1 & 2 & 3 & 4 & 5 & 6 & 7
\end{tabular}

Generally, in stressful situations...

\begin{tabular}{|c|c|c|c|c|c|c|c|}
\hline $\begin{array}{l}\text { 1. I weigh out many options before choosing } \\
\text { how to take action. }\end{array}$ & 1 & 2 & 3 & 4 & 5 & 6 & 7 \\
\hline 2. I can't focus on anything when I am upset. & 1 & 2 & 3 & 4 & 5 & 6 & 7 \\
\hline $\begin{array}{l}\text { 3. It's hard to think of different ways of dealing } \\
\text { with the situation }\end{array}$ & 1 & 2 & 3 & 4 & 5 & 6 & 7 \\
\hline $\begin{array}{l}\text { 4. I control my thoughts and feelings by putting } \\
\text { the situation in context. }\end{array}$ & 1 & 2 & 3 & 4 & 5 & 6 & 7 \\
\hline $\begin{array}{l}\text { 5. I anticipate the consequences of my actions } \\
\text { before acting. }\end{array}$ & 1 & 2 & 3 & 4 & 5 & 6 & 7 \\
\hline $\begin{array}{l}\text { 6. I can remain in control over my thoughts and } \\
\text { emotions. }\end{array}$ & 1 & 2 & 3 & 4 & 5 & 6 & 7 \\
\hline $\begin{array}{l}\text { 7. It's difficult let go of intrusive thoughts or } \\
\text { emotions. }\end{array}$ & 1 & 2 & 3 & 4 & 5 & 6 & 7 \\
\hline $\begin{array}{l}\text { 8. It's hard for me to put things in perspective } \\
\text { when I'm upset. }\end{array}$ & 1 & 2 & 3 & 4 & 5 & 6 & 7 \\
\hline $\begin{array}{l}\text { 9. My thoughts and emotions become too much } \\
\text { to deal with. }\end{array}$ & 1 & 2 & 3 & 4 & 5 & 6 & 7 \\
\hline 10. I have a hard time managing my emotions. & 1 & 2 & 3 & 4 & 5 & 6 & 7 \\
\hline $\begin{array}{l}\text { 11. I take the time to see things from different } \\
\text { perspectives before reacting. }\end{array}$ & 1 & 2 & 3 & 4 & 5 & 6 & 7 \\
\hline $\begin{array}{l}\text { 12. I keep playing the situation over and over } \\
\text { again in my head }\end{array}$ & 1 & 2 & 3 & 4 & 5 & 6 & 7 \\
\hline
\end{tabular}


13. I consider the situation for multiple

$\begin{array}{lllllll}1 & 2 & 3 & 4 & 5 & 6 & 7\end{array}$
viewpoints before responding.

\begin{tabular}{|c|c|c|c|c|c|c|c|}
\hline $\begin{array}{l}\text { 14. I feel like I lose control over my thoughts and } \\
\text { emotions. }\end{array}$ & 1 & 2 & 3 & 4 & 5 & 6 & 7 \\
\hline 15. It's hard for me to shift my attent & 1 & 2 & 3 & 4 & 5 & 6 & 7 \\
\hline
\end{tabular}
from negative thoughts or feelings.

$\begin{aligned} & \text { 16. I find it easy to look for something positive, } \\ & \text { even when I am stressed. }\end{aligned}$
$\begin{aligned} & \text { 17. I control negative thoughts and emotions by } \\ & \text { 17 }\end{aligned}$
modifying the way I think about the situation.
18. I get rid of negative thinking by re-evaluating $\quad \begin{array}{lllllllll}1 & 2 & 3 & 4 & 5 & 6 & 7\end{array}$ the situation.
19. I think of a plan of what to do best before $\quad \begin{array}{lllllllll}1 & 2 & 3 & 4 & 5 & 6 & 7\end{array}$ acting.
20. It is easy for me to ignore distracting $\quad \begin{array}{lllllllll} & 1 & 2 & 3 & 4 & 5 & 6 & 7\end{array}$ thoughts.
21. It's hard for me to ignore negative emotions $\quad \begin{array}{lllllllll}1 & 2 & 3 & 4 & 5 & 6 & 7\end{array}$ once they have been provoked.
22. I can think of multiple coping options before $\quad \begin{array}{lllllllll}1 & 2 & 3 & 4 & 5 & 6 & 7\end{array}$ deciding how to respond.
23. I look for many strategies of dealing with the $\quad \begin{array}{lllllllll}1 & 2 & 3 & 4 & 5 & 6 & 7\end{array}$ situation.

24. I get rid of negative emotions by changing the
way I think about the situation.
25. I play out the consequences of various actions
2
before choosing the best one.

\begin{tabular}{|c|c|c|c|c|c|c|c|}
\hline $\begin{array}{l}\text { 26. I get easily distracted by upsetting thoughts } \\
\text { or feelings. }\end{array}$ & 1 & 2 & 3 & 4 & 5 & 6 & 7 \\
\hline 27. I approach the situation from multiple angles. & 1 & 2 & 3 & 4 & 5 & 6 & 7 \\
\hline $\begin{array}{l}\text { 28. My thoughts and emotions interfere with my } \\
\text { ability to concentrate. }\end{array}$ & 1 & 2 & 3 & 4 & 5 & 6 & 7 \\
\hline $\begin{array}{l}\text { 29. I take the time to think of more than one way } \\
\text { to resolve the problem. }\end{array}$ & 1 & 2 & 3 & 4 & 5 & 6 & 7 \\
\hline
\end{tabular}


30. It is easy for me to shift my attention to other

$\begin{array}{lllllll}1 & 2 & 3 & 4 & 5 & 6 & 7\end{array}$
things if I am upset.

31. I can easily deal with my thoughts and

$\begin{array}{lllllll}1 & 2 & 3 & 4 & 5 & 6 & 7\end{array}$
feelings.

$\begin{aligned} & \text { 32. I play out the situation in my mind before } \\ & \text { responding. }\end{aligned}$
$\begin{aligned} & \text { 33. I manage my thoughts or feelings by } \\ & \text { reframing the situation. }\end{aligned}$

$\begin{aligned} & \text { 34. I find it difficult to think of many options for } \\ & \text { resolving the situation. }\end{aligned}$
$\begin{aligned} & \text { 35. The same thoughts keep going through my } \\ & \text { mind again and again. }\end{aligned}$

36. I can't stop dwelling on my feelings. $\quad \begin{array}{lllllllll}7 & 2 & 3 & 4 & 5 & 6 & 7\end{array}$

37. My thoughts repeat themselves over and over $\quad \begin{array}{lllllllll}1 & 2 & 3 & 4 & 5 & 6 & 7\end{array}$ again.
38. Putting a positive spin on a bad experience $\quad \begin{array}{lllllllll}1 & 2 & 3 & 4 & 5 & 6\end{array}$ comes fairly easy to me.
39. I have difficulty controlling my thoughts and $\quad \begin{array}{lllllllll}1 & 2 & 3 & 4 & 5 & 6 & 7\end{array}$ emotions.

\begin{tabular}{|c|c|c|c|c|c|c|c|}
\hline $\begin{array}{l}\text { 40. I can't think about anything else except for } \\
\text { the situation. }\end{array}$ & 1 & 2 & 3 & 4 & 5 & 6 & 7 \\
\hline $\begin{array}{l}\text { 41. I find it easy to set-aside unpleasant thought } \\
\text { or emotions. }\end{array}$ & 1 & 2 & 3 & 4 & 5 & 6 & 7 \\
\hline $\begin{array}{l}\text { 42. It is easy for me to reassess a negative } \\
\text { experience into a positive one. }\end{array}$ & 1 & 2 & 3 & 4 & 5 & 6 & 7 \\
\hline 43. I can easily to suppress upsetting memories. & 1 & 2 & 3 & 4 & 5 & 6 & 7 \\
\hline $\begin{array}{l}\text { 44. I take the time to think of several ways to best } \\
\text { cope with the situation before acting. }\end{array}$ & 1 & 2 & 3 & 4 & 5 & 6 & 7 \\
\hline
\end{tabular}




\section{Stress Appraisal Measure (SAM) - for studies 1 and 4}

This questionnaire is concerned with your thoughts about various aspects of the situation you have just experienced. Specifically, the employment task. There are no right or wrong answers. Please respond according to how you view this situation right NOW. Please answer ALL questions. Answer each question by CIRCLING the appropriate number corresponding to the following scale.

$\begin{array}{rrrcc}1 & 2 & 3 & 4 & 5 \\ \text { Not at all } & \text { Slightly } & \text { Moderately } & \text { Considerably } & \text { Extremely }\end{array}$

1. Was this a totally hopeless situation?

$\begin{array}{lllll}1 & 2 & 3 & 4 & 5\end{array}$

2. Did this situation create tension in me?

$\begin{array}{lllll}1 & 2 & 3 & 4 & 5\end{array}$

3. Was the outcome of this situation uncontrollable by anyone? $\quad \begin{array}{llllll}1 & 2 & 3 & 4 & 5\end{array}$

4. Was there someone or some agency you can turn to for

help if I need it?

5. Did this situation make you feel anxious?

6. Did this situation have important consequences for you?

7. Was this going to have a positive impact on you?

8. How eager were you to tackle this problem?

9. How much will you be affected by the outcome of this situation?

10. To what extent can you become a stronger person because

$\begin{array}{lllll}1 & 2 & 3 & 4 & 5 \\ 1 & 2 & 3 & 4 & 5 \\ 1 & 2 & 3 & 4 & 5 \\ 1 & 2 & 3 & 4 & 5 \\ 1 & 2 & 3 & 4 & 5\end{array}$

of this problem?

11. Will the outcome of this situation be negative?

12. Did you have the ability to do well in this situation?

13. Did this situation have serious implications for you? 


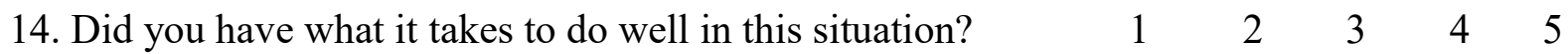

15. Was there help available to you for dealing with this problem? $\quad \begin{array}{llllll}2 & 2 & 3 & 4 & 5\end{array}$

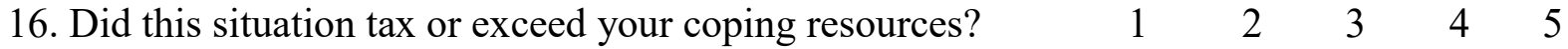

17. Are there sufficient resources available to help you in dealing? $1 \quad \begin{array}{lllll}2 & 3 & 4 & 5\end{array}$

18. Is this beyond anyone's power to do anything about this situation?

$\begin{array}{lllll}1 & 2 & 3 & 4 & 5\end{array}$

19. To what extent were you excited thinking about the outcome of this situation?

$\begin{array}{lllll}1 & 2 & 3 & 4 & 5\end{array}$

20. How threatening is this situation?

$\begin{array}{lllll}1 & 2 & 3 & 4 & 5\end{array}$

21. Is the problem unresolvable by anyone?

$\begin{array}{lllll}1 & 2 & 3 & 4 & 5\end{array}$

22. Will you be able to overcome the problem?

$\begin{array}{lllll}1 & 2 & 3 & 4 & 5\end{array}$

23. Is there anyone who can help you manage the problem?

$\begin{array}{lllll}1 & 2 & 3 & 4 & 5\end{array}$

24. To what extent did you perceive this situation as stressful? $\quad \begin{array}{lllllll}1 & 2 & 3 & 4 & 5\end{array}$

25. Did you have the skills necessary to achieve a successful outcome to this situation?

$\begin{array}{lllll}1 & 2 & 3 & 4 & 5\end{array}$

24. To what extent did this event require coping efforts

$\begin{array}{llllllll}\text { on your part? } & 1 & 2 & 3 & 4 & 5\end{array}$

27. Did this situation have long-term consequences for you? $\quad \begin{array}{llllll}1 & 2 & 3 & 4 & 5\end{array}$

28. Is this going to have a negative impact on you? $\quad 1 \quad \begin{array}{lllll}2 & 3 & 4 & 5\end{array}$ 


\section{Stress Appraisal Measure (SAM) - for study 3C}

This questionnaire is concerned with your thoughts about various aspects of the situation identified previously. There are no right or wrong answers. Please respond according to how you view this situation right NOW. Please answer ALL questions. Answer each question by CIRCLING the appropriate number corresponding to the following scale.

$\begin{array}{ccccc}1 & 2 & 3 & 4 & 5 \\ \text { Not at all } & \text { Slightly } & \text { Moderately } & \text { Considerably } & \text { Extremely }\end{array}$

1. Is this a totally hopeless situation?

2. Does this situation create tension in me?

3. Is the outcome of this situation uncontrollable by anyone?

4. Is there someone or some agency I can turn to for help if I need it?

5. Does this situation make me feel anxious?

6. Does this situation have important consequences for me?

7. Is this going to have a positive impact in me?

8. How eager am I to tackle this problem?

9. How much will I be affected by the outcome of this situation?

10. To what extent can I become a stronger person because of this problem?

11. Will the outcome of this situation be negative?

12. Do I have the ability to do well in this situation?

13. Does this situation have serious implications for me?

14. Do I have what it takes to do well in this situation?

15. Is there help available to me for dealing with this problem?

16. Does this situation tax or exceed my coping resources?

17. Are there sufficient resources available to help me in dealing?

18. Is this beyond anyone's power to do anything about this situation?

$\begin{array}{lllll}1 & 2 & 3 & 4 & 5 \\ 1 & 2 & 3 & 4 & 5 \\ 1 & 2 & 3 & 4 & 5\end{array}$

$\begin{array}{lllll}1 & 2 & 3 & 4 & 5\end{array}$

$\begin{array}{lllll}1 & 2 & 3 & 4 & 5\end{array}$

$\begin{array}{lllll}1 & 2 & 3 & 4 & 5\end{array}$

$\begin{array}{lllll}1 & 2 & 3 & 4 & 5\end{array}$

$\begin{array}{lllll}1 & 2 & 3 & 4 & 5\end{array}$

$\begin{array}{lllll}1 & 2 & 3 & 4 & 5\end{array}$

$\begin{array}{lllll}1 & 2 & 3 & 4 & 5\end{array}$

$\begin{array}{lllll}1 & 2 & 3 & 4 & 5\end{array}$

$\begin{array}{lllll}1 & 2 & 3 & 4 & 5\end{array}$

$\begin{array}{lllll}1 & 2 & 3 & 4 & 5\end{array}$

$\begin{array}{lllll}1 & 2 & 3 & 4 & 5\end{array}$

$\begin{array}{lllll}1 & 2 & 3 & 4 & 5\end{array}$

$\begin{array}{lllll}1 & 2 & 3 & 4 & 5\end{array}$

$\begin{array}{lllll}1 & 2 & 3 & 4 & 5\end{array}$

$\begin{array}{lllll}1 & 2 & 3 & 4 & 5\end{array}$

19. To what extent am I excited thinking about the outcome of this 
situation?

20. How threatening is this situation?

21. Is the problem unresolvable by anyone?

22. Will I be able to overcome the problem?

23. Is there anyone who can help me manage the problem?

24. To what extent do I perceive this situation as stressful?

25. Do I have the skills necessary to achieve a successful outcome to this situation?

26. To what extent does this event require coping efforts on my part?

27. Does this situation have long-term consequences for me?

28. Is this going to have a negative impact on me?

$\begin{array}{lllll}1 & 2 & 3 & 4 & 5 \\ 1 & 2 & 3 & 4 & 5 \\ 1 & 2 & 3 & 4 & 5 \\ 1 & 2 & 3 & 4 & 5 \\ 1 & 2 & 3 & 4 & 5\end{array}$

$\begin{array}{lllll}1 & 2 & 3 & 4 & 5\end{array}$

$\begin{array}{lllll}1 & 2 & 3 & 4 & 5\end{array}$

$\begin{array}{lllll}1 & 2 & 3 & 4 & 5\end{array}$

$\begin{array}{lllll}1 & 2 & 3 & 4 & 5\end{array}$

$\begin{array}{lllll}1 & 2 & 3 & 4 & 5\end{array}$




\section{Positive and negative Affect Schedule 20 item (PANAS-20)}

This scale consists of a number of words that describe different feelings and emotions. Read each item and then list the number from the scale below next to each word.

Indicate to what extent you feel this way right now.

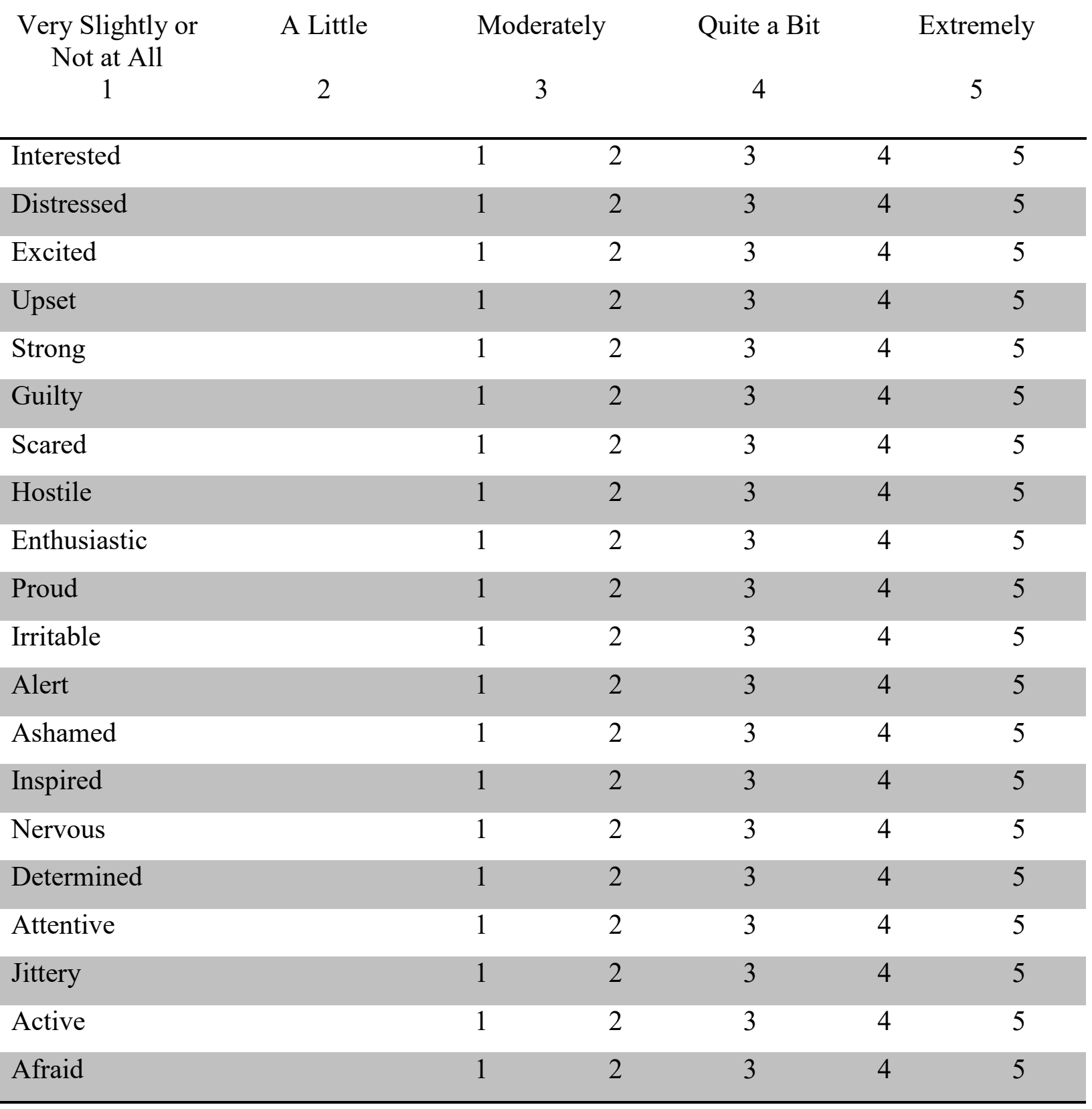




\section{Positive and Negative Affect Schedule - Expanded Version (PANAS-X)}

Using the rating scale beside each item, please indicate how much each adjective describes how you feel at the moment. There are no right or wrong answers, we just want you to be as honest as possible in indicating how you're feeling right now.

$\begin{array}{llllllllll}\text { Active } & \text { Not at all } & 0 & 1 & 2 & 3 & 4 & 5 & 6 & \text { Extremely } \\ \text { Afraid } & \text { Not at all } & 0 & 1 & 2 & 3 & 4 & 5 & 6 & \text { Extremely } \\ \text { Alert } & \text { Not at all } & 0 & 1 & 2 & 3 & 4 & 5 & 6 & \text { Extremely } \\ \text { Angry } & \text { Not at all } & 0 & 1 & 2 & 3 & 4 & 5 & 6 & \text { Extremely } \\ \text { Annoyed } & \text { Not at all } & 0 & 1 & 2 & 3 & 4 & 5 & 6 & \text { Extremely } \\ \text { Anxious } & \text { Not at all } & 0 & 1 & 2 & 3 & 4 & 5 & 6 & \text { Extremely } \\ \text { Ashamed } & \text { Not at all } & 0 & 1 & 2 & 3 & 4 & 5 & 6 & \text { Extremely } \\ \text { Attentive } & \text { Not at all } & 0 & 1 & 2 & 3 & 4 & 5 & 6 & \text { Extremely } \\ \text { Confused } & \text { Not at all } & 0 & 1 & 2 & 3 & 4 & 5 & 6 & \text { Extremely } \\ \text { Contempt } & \text { Not at all } & 0 & 1 & 2 & 3 & 4 & 5 & 6 & \text { Extremely } \\ \text { Depressed } & \text { Not at all } & 0 & 1 & 2 & 3 & 4 & 5 & 6 & \text { Extremely } \\ \text { Determined } & \text { Not at all } & 0 & 1 & 2 & 3 & 4 & 5 & 6 & \text { Extremely } \\ \text { Disdain } & \text { Not at all } & 0 & 1 & 2 & 3 & 4 & 5 & 6 & \text { Extremely } \\ \text { Disgust } & \text { Not at all } & 0 & 1 & 2 & 3 & 4 & 5 & 6 & \text { Extremely } \\ \text { Distressed } & \text { Not at all } & 0 & 1 & 2 & 3 & 4 & 5 & 6 & \text { Extremely } \\ \text { Embarrassed } & \text { Not at all } & 0 & 1 & 2 & 3 & 4 & 5 & 6 & \text { Extremely } \\ \text { Enraged } & \text { Not at all } & 0 & 1 & 2 & 3 & 4 & 5 & 6 & \text { Extremely } \\ \text { Enthusiastic } & \text { Not at all } & 0 & 1 & 2 & 3 & 4 & 5 & 6 & \text { Extremely } \\ \text { Excited } & \text { Not at all } & 0 & 1 & 2 & 3 & 4 & 5 & 6 & \text { Extremely } \\ \text { Frustrated } & \text { Not at all } & 0 & 1 & 2 & 3 & 4 & 5 & 6 & \text { Extremely } \\ \text { Guilty } & \text { Not at all } & 0 & 1 & 2 & 3 & 4 & 5 & 6 & \text { Extremely } \\ \text { Happy } & \text { Not at all } & 0 & 1 & 2 & 3 & 4 & 5 & 6 & \text { Extremely } \\ \text { Helpless } & \text { Not at all } & 0 & 1 & 2 & 3 & 4 & 5 & 6 & \text { Extremely }\end{array}$




\begin{tabular}{|c|c|c|c|c|c|c|c|c|c|}
\hline Hostile & Not at all & 0 & 1 & 2 & 3 & 4 & 5 & 6 & Extremely \\
\hline Humiliated & Not at all & 0 & 1 & 2 & 3 & 4 & 5 & 6 & Extremely \\
\hline Indifferent & Not at all & 0 & 1 & 2 & 3 & 4 & 5 & 6 & Extremely \\
\hline Infuriated & Not at all & 0 & 1 & 2 & 3 & 4 & 5 & 6 & Extremely \\
\hline Inspired & Not at all & 0 & 1 & 2 & 3 & 4 & 5 & 6 & Extremely \\
\hline Interested & Not at all & 0 & 1 & 2 & 3 & 4 & 5 & 6 & Extremely \\
\hline Irritable & Not at all & 0 & 1 & 2 & 3 & 4 & 5 & 6 & Extremely \\
\hline Jittery & Not at all & 0 & 1 & 2 & 3 & 4 & 5 & 6 & Extremely \\
\hline Nervous & Not at all & 0 & 1 & 2 & 3 & 4 & 5 & 6 & Extremely \\
\hline Proud & Not at all & 0 & 1 & 2 & 3 & 4 & 5 & 6 & Extremely \\
\hline Regretful & Not at all & 0 & 1 & 2 & 3 & 4 & 5 & 6 & Extremely \\
\hline Responsible & Not at all & 0 & 1 & 2 & 3 & 4 & 5 & 6 & Extremely \\
\hline $\mathrm{Sad}$ & Not at all & 0 & 1 & 2 & 3 & 4 & 5 & 6 & Extremely \\
\hline Scared & Not at all & 0 & 1 & 2 & 3 & 4 & 5 & 6 & Extremely \\
\hline Strong & Not at all & 0 & 1 & 2 & 3 & 4 & 5 & 6 & Extremely \\
\hline Unhappy & Not at all & 0 & 1 & 2 & 3 & 4 & 5 & 6 & Extremely \\
\hline Upset & Not at all & 0 & 1 & 2 & 3 & 4 & 5 & 6 & Extremely \\
\hline Worried & Not at all & 0 & 1 & 2 & 3 & 4 & 5 & 6 & Extremely \\
\hline
\end{tabular}




\section{Beck Depression Inventory (BDI)}

On this questionnaire are groups of statements. Please read the entire group of statements in each category. Then pick out ONE statement in that group which best describes the way you feel. Check off the number beside the statement you have chosen.

1. _ $\quad 0=$ I do not feel sad

$1=$ I feel sad or blue

_ $2 \mathrm{a}=\mathrm{I}$ am blue or sad all of the time and I can't snap out of it

$2 \mathrm{~b}=\mathrm{I}$ am so sad or unhappy that it is very painful

$3=\mathrm{I}$ am so sad or unhappy that I can't stand it

2. _ $0=$ I am not particularly pessimistic or discouraged about the future

$1=$ I feel discouraged about the future

$2 \mathrm{a}=\mathrm{I}$ feel I have nothing to look forward to

$2 \mathrm{~b}=\mathrm{I}$ feel I won't every get over my troubles

$3=$ I feel that the future is hopeless and things cannot improve

3. 0 _ 0 I do not feel like a failure

$1=$ I feel I have failed more than the average person

$2 \mathrm{a}=\mathrm{I}$ feel I have accomplished very little that is worthwhile or that means anything

$2 \mathrm{~b}=$ As I look back on my life, all I can see is a lot of failures

$3=$ I feel I am a complete failure as a person

4. $\_$_ $0=$ I am not particularly dissatisfied

$1 \mathrm{a}=\mathrm{I}$ feel bored most of the time

$1 \mathrm{~b}=\mathrm{I}$ don't enjoy things the way I used to

2 _ 2 I don't get satisfaction out of anything anymore

$3=$ I am dissatisfied with everything

5. _ 0 _ 0 I don't feel particularly guilty

$1=$ I feel bad or unworthy a good part of the time

$2 \mathrm{a}=\mathrm{I}$ feel quite guilty

$2 \mathrm{~b}=\mathrm{I}$ feel bad or unworthy practically of the time now

$3=$ I feel as though I am very bad or worthless

6. _ $0=$ I don't feel I am being punished

1 - I have a feeling that something bad may happen to me

$2=$ I feel I am being punished or will be punished

$3 \mathrm{a}=\mathrm{I}$ feel I deserve to be punished

$3 b=$ I want to be punished

7. $0=$ I don't feel disappointed in myself

$1 \mathrm{a}=\mathrm{I}$ am disappointed in myself

$1 \mathrm{~b}=$ I don't like myself 
$2=$ I am disgusted with myself

$3=$ I hate myself

8. $\quad 0 \quad$ _ $\quad$ I do not feel I am any worse than anybody else

$1=$ I am very critical of myself for my weaknesses or mistakes

_ $2 \mathrm{a}=$ I blame myself for everything that goes wrong

$2 \mathrm{~b}=\mathrm{I}$ feel I have many bad faults

9. $0=1$ don't have thoughts of harming myself

$1=$ I have thoughts of harming myself but I would not carry them out

$2 \mathrm{a}=\mathrm{I}$ feel I would be better off dead

$2 \mathrm{~b}=\mathrm{I}$ have definite plans about committing suicide

$2 \mathrm{c}=\mathrm{I}$ feel my family would be better off if I were dead

3 = I would kill myself if I could

10. $\quad 0=$ I don't cry anymore than usual

$1=\mathrm{I}$ cry more now than I used to

$2=\mathrm{I}$ cry all the time now. I can't stop it

$3=$ I used to be able to cry but now I can't cry at all even though I want to

11. $0=$ I am no more irritable than usual

$1=$ I am more irritable than usual

$2=$ I am much more irritable than usual

$3=$ I am irritable all the time

12. _ $0=$ I have not lost interest in other people

$1=\mathrm{I}$ am less interested in other people than I used to be

2 - I have lost most of my interest in other people and I have little feeling for them

3 = I have lost all my interest in other people and don't care about them at all

13. $0=$ I make decisions about as well as ever

$1=\mathrm{I}$ am less sure of myself now and try to put off making decisions

2 - I can't make decisions anymore without help

$3=$ I can't make decisions at all anymore

14. $0=$ I don't feel I look any worse than I used to

$1=\mathrm{I}$ am worried that I am looking old or unattractive

$2=$ I feel that there are permanent changes in my appearance and they make me look unattractive

$3=$ I feel that I am ugly or repulsive looking

15.__ 0 _ I can work about as well as before

$1 \mathrm{a}=$ It takes extra effort to get started at doing something

$1 \mathrm{~b}=\mathrm{I}$ don't work as well as I used to

2 - I have to push myself very hard to do anything

3 = I can't do any work at all 
16. _ $0=$ I can sleep as well as usual

$1=$ I wake up more tired in the morning than I used to

2 = I wake up 1-2 hours earlier than usual and find it hard to get back to sleep

3 = I wake up early every day and can't get more than 5 hours sleep

17. 0 _ 0 I don't get any more tired than usual

$1=$ I get tired more easily than I used to

$2=$ I get tired from doing anything

3 = I get too tired to do anything

18a. __ $0=$ My appetite is no worse than usual

$1=$ My appetite is not as good as it used to be

2 = My appetite is much worse now

3 = I have no appetite at all any more

18b. __ $0=\mathrm{I}$ am not eating more than usual

$1=$ I am eating a little more than usual

$2=$ I am eating somewhat more than usual

$3=$ I am eating a lot more than usual

18c. $0=$ I have had no change in food preferences lately

$1=$ I have been craving more carbohydrates (starches or sweets lately)

$2=\mathrm{I}$ have had irresistible craving for sweets and starches lately

19a. _ $0=$ I haven't lost much weight, if any, lately

$1=$ I have lost more than 5 pounds

$2=$ I have lost more than 10 pounds

3 = I have lost more than 15 pounds

19b. _ 0 = I have not gained any weight lately

$1=$ I have gained more than 5 pounds

$2=$ I have gained more than 10 pounds

3 = I have gained more than 15 pounds

20. _ $0=$ I am no more concerned about my health than usual

$1=\mathrm{I}$ am concerned about aches and pains or upset stomach or constipation or other unpleasant feelings in my body

2 = I am so concerned with how I feel or what I feel that it's hard to think of much else

3 = I am completely absorbed in what I feel

21. $0=$ I have not noticed any recent change in my interest in sex

$1=\mathrm{I}$ am less interested in sex than I used to be

$2=\mathrm{I}$ am much less interested in sex now 
$3=$ I have lost interest in sex completely

22. _ $0=$ I have not had any increase in nightly sleep length lately

$1=\mathrm{I}$ have had at least 1-hour increase in sleep length

$2=$ I have had at least 2-hour increase in sleep length

$3=\mathrm{I}$ have had at least 3-hour increase in sleep length

My average nightly sleep length in the past week is:

hours

23. $0=\mathrm{I}$ am not feeling more fatigued than usual

$1=\mathrm{I}$ feel more fatigued than usual lately, but it does not interfere with my daily functioning

2 = I feel more fatigued than usual lately, and it interferes somewhat with my daily functioning

3 = I feel more fatigued than usual lately, and it significantly interferes with my daily functioning

24. _ $0=$ I have not had any mood swings or slumps lately

$1=$ I have had some mood swings or slumps lately but very minor

$2=\mathrm{I}$ have had more mood swings or slumps than usual

3 = I have had severe mood swings or slumps lately 


\section{Early Trauma Inventory - Short Report (ETI-SR)}

Most people have experienced a traumatic event at some point in their life. For this survey, we are interested in the types of traumatic events that you may have experienced, and how old you were when you experienced it. For each question, please indicate how many times you have experienced the event (if at all), and at what age range, by circling the appropriate number.

Part 1. General Traumas

\section{Were you ever exposed to a life-threatening natural disaster?}

\begin{tabular}{l|c|c|c|c|c|c}
\hline Age 0 to 5 & $\begin{array}{c}0 \\
\text { Never }\end{array}$ & $\begin{array}{c}1 \\
\text { Once }\end{array}$ & $\begin{array}{c}2 \\
2-3 \text { times }\end{array}$ & $\begin{array}{c}3 \\
4-5 \text { times }\end{array}$ & $\begin{array}{c}4 \\
6-10 \\
\text { times }\end{array}$ & More than 10 times \\
\hline Age 6 to 12 & $\begin{array}{c}0 \\
\text { Never }\end{array}$ & $\begin{array}{c}1 \\
\text { Once }\end{array}$ & $\begin{array}{c}2 \\
2-3 \text { times }\end{array}$ & $\begin{array}{c}3 \\
4-5 \text { times }\end{array}$ & $\begin{array}{c}4 \\
6-10 \\
\text { times }\end{array}$ & $\begin{array}{c}5 \\
\text { More than } 10 \text { times }\end{array}$ \\
\hline Age 13 to 18 & $\begin{array}{c}1 \\
\text { Never }\end{array}$ & $\begin{array}{c}1 \\
\text { Once }\end{array}$ & $\begin{array}{c}2 \\
2-3 \text { times }\end{array}$ & $\begin{array}{c}3 \\
4-5 \text { times }\end{array}$ & $\begin{array}{c}4 \\
6-10 \\
\text { times }\end{array}$ & More than 10 times \\
\hline Age 18+ & $\begin{array}{c}1 \\
\text { Never }\end{array}$ & Once & $\begin{array}{c}2 \\
2-3 \text { times }\end{array}$ & $\begin{array}{c}3 \\
4-5 \text { times } \\
6-10 \\
\text { times }\end{array}$ & More than 10 times \\
\hline
\end{tabular}

\section{Were you involved in a serious accident?}

\begin{tabular}{l|c|c|c|c|c|c}
\hline Age 0 to 5 & $\begin{array}{c}0 \\
\text { Never }\end{array}$ & $\begin{array}{c}1 \\
\text { Once }\end{array}$ & $\begin{array}{c}2 \\
2-3 \text { times }\end{array}$ & $\begin{array}{c}3 \\
4-5 \text { times }\end{array}$ & $\begin{array}{c}4 \\
6-10 \\
\text { times }\end{array}$ & More than 10 times \\
\hline Age 6 to 12 & $\begin{array}{c}0 \\
\text { Never }\end{array}$ & $\begin{array}{c}1 \\
\text { Once }\end{array}$ & $\begin{array}{c}2 \\
2-3 \text { times }\end{array}$ & $\begin{array}{c}3 \\
4-5 \text { times }\end{array}$ & $\begin{array}{c}4 \\
6-10 \\
\text { times }\end{array}$ & $\begin{array}{c}5 \\
\text { More than } 10 \text { times }\end{array}$ \\
\hline Age 13 to 18 & 0 & $\begin{array}{c}1 \\
\text { Once }\end{array}$ & $\begin{array}{c}2 \\
2-3 \text { times }\end{array}$ & $\begin{array}{c}3 \\
4-5 \text { times }\end{array}$ & $\begin{array}{c}4 \\
6-10 \\
\text { times }\end{array}$ & More than 10 times \\
\hline Age 18+ & $\begin{array}{c}1 \\
\text { Never }\end{array}$ & Once & $\begin{array}{c}2 \\
2-3 \text { times }\end{array}$ & $\begin{array}{c}3 \\
4-5 \text { times } \\
6-10 \\
\text { times }\end{array}$ & More than 10 times \\
\hline
\end{tabular}

\section{Did you ever suffer a serious personal injury or illness?}

\begin{tabular}{l|c|c|c|c|c|c}
\hline Age 0 to 5 & $\begin{array}{c}0 \\
\text { Never }\end{array}$ & $\begin{array}{c}1 \\
\text { Once }\end{array}$ & $\begin{array}{c}2 \\
2-3 \text { times }\end{array}$ & $\begin{array}{c}3 \\
4-5 \text { times }\end{array}$ & $\begin{array}{c}4 \\
6-10 \\
\text { times }\end{array}$ & More than 10 times \\
\hline Age 6 to 12 & $\begin{array}{c}0 \\
\text { Never }\end{array}$ & $\begin{array}{c}1 \\
\text { Once }\end{array}$ & $\begin{array}{c}2 \\
2-3 \text { times }\end{array}$ & $\begin{array}{c}3 \\
4-5 \text { times }\end{array}$ & $\begin{array}{c}4 \\
6-10 \\
\text { times }\end{array}$ & $\begin{array}{c}5 \\
\text { More than 10 times }\end{array}$ \\
\hline Age 13 to 18 & $\begin{array}{c}1 \\
\text { Never }\end{array}$ & $\begin{array}{c}1 \\
\text { Once }\end{array}$ & $\begin{array}{c}2 \\
2-3 \text { times }\end{array}$ & $\begin{array}{c}3 \\
4-5 \text { times }\end{array}$ & $\begin{array}{c}4 \\
6-10 \\
\text { times }\end{array}$ & More than 10 times
\end{tabular}




\begin{tabular}{|c|c|c|c|c|c|c|}
\hline Age $18+$ & $\begin{array}{c}0 \\
\text { Never }\end{array}$ & $\begin{array}{c}1 \\
\text { Once }\end{array}$ & $\begin{array}{c}2 \\
2-3 \text { times }\end{array}$ & $\begin{array}{c}3 \\
4-5 \text { times }\end{array}$ & $\begin{array}{c}4 \\
6-10 \\
\text { times }\end{array}$ & $\begin{array}{l}5 \\
\text { More than } 10 \text { times }\end{array}$ \\
\hline \multicolumn{7}{|c|}{ 4. Did you ever experience the death or serious illness of a parent or a primary caretaker? } \\
\hline Age 0 to 5 & $\begin{array}{c}0 \\
\text { Never }\end{array}$ & $\begin{array}{c}1 \\
\text { Once }\end{array}$ & $\begin{array}{c}2 \\
2-3 \text { times }\end{array}$ & $\begin{array}{c}3 \\
4-5 \text { times }\end{array}$ & $\begin{array}{c} \\
6-10 \\
\text { times }\end{array}$ & $\begin{array}{c}5 \\
\text { More than } 10 \text { times }\end{array}$ \\
\hline Age 6 to 12 & $\begin{array}{c}0 \\
\text { Never }\end{array}$ & $\begin{array}{c}1 \\
\text { Once }\end{array}$ & $\begin{array}{c}2 \\
2-3 \text { times }\end{array}$ & $\begin{array}{c}3 \\
4-5 \text { times }\end{array}$ & $\begin{array}{c}4 \\
6-10 \\
\text { times } \\
\end{array}$ & $\begin{array}{c}5 \\
\text { More than } 10 \text { times }\end{array}$ \\
\hline Age 13 to 18 & $\begin{array}{c}0 \\
\text { Never }\end{array}$ & $\begin{array}{c}1 \\
\text { Once }\end{array}$ & $\begin{array}{c}2 \\
2-3 \text { times }\end{array}$ & $\begin{array}{c}3 \\
4-5 \text { times }\end{array}$ & $\begin{array}{c}4 \\
6-10 \\
\text { times } \\
\end{array}$ & $\begin{array}{c}5 \\
\text { More than } 10 \text { times }\end{array}$ \\
\hline Age $18+$ & $\begin{array}{c}0 \\
\text { Never }\end{array}$ & $\begin{array}{c}1 \\
\text { Once }\end{array}$ & $\begin{array}{c}2 \\
2-3 \text { times }\end{array}$ & $\begin{array}{c}3 \\
4-5 \text { times }\end{array}$ & $\begin{array}{c}4 \\
6-10 \\
\text { times } \\
\end{array}$ & $\begin{array}{c}5 \\
\text { More than } 10 \text { times }\end{array}$ \\
\hline \multicolumn{7}{|c|}{ 5. Did you experience the divorce or separation of your parents? } \\
\hline Age 0 to 5 & $\begin{array}{c}0 \\
\text { Never }\end{array}$ & $\begin{array}{c}1 \\
\text { Once }\end{array}$ & $\begin{array}{c}2 \\
2-3 \text { times }\end{array}$ & $\begin{array}{c}3 \\
4-5 \text { times }\end{array}$ & $\begin{array}{c} \\
6-10 \\
\text { times }\end{array}$ & $\begin{array}{c}5 \\
\text { More than } 10 \text { times }\end{array}$ \\
\hline Age 6 to 12 & $\begin{array}{c}0 \\
\text { Never }\end{array}$ & $\begin{array}{c}1 \\
\text { Once }\end{array}$ & $\begin{array}{c}2 \\
2-3 \text { times }\end{array}$ & $\begin{array}{c}3 \\
4-5 \text { times }\end{array}$ & $\begin{array}{c}4 \\
6-10 \\
\text { times } \\
\end{array}$ & $\begin{array}{c}5 \\
\text { More than } 10 \text { times }\end{array}$ \\
\hline Age 13 to 18 & $\begin{array}{c}0 \\
\text { Never }\end{array}$ & $\begin{array}{c}1 \\
\text { Once }\end{array}$ & $\begin{array}{c}2 \\
2-3 \text { times }\end{array}$ & $\begin{array}{c}3 \\
4-5 \text { times }\end{array}$ & $\begin{array}{c}4 \\
6-10 \\
\text { times }\end{array}$ & $\begin{array}{c}5 \\
\text { More than } 10 \text { times }\end{array}$ \\
\hline Age $18+$ & $\begin{array}{c}0 \\
\text { Never }\end{array}$ & $\begin{array}{c}1 \\
\text { Once }\end{array}$ & $\begin{array}{c}2 \\
2-3 \text { times }\end{array}$ & $\begin{array}{c}3 \\
4-5 \text { times }\end{array}$ & $\begin{array}{c}4 \\
6-10 \\
\text { times } \\
\end{array}$ & $\begin{array}{c}5 \\
\text { More than } 10 \text { times }\end{array}$ \\
\hline
\end{tabular}

6. Did you experience the death or serious injury of a sibling?

\begin{tabular}{l|c|c|c|c|c|c}
\hline Age 0 to 5 & $\begin{array}{c}0 \\
\text { Never }\end{array}$ & $\begin{array}{c}1 \\
\text { Once }\end{array}$ & $\begin{array}{c}2 \\
2-3 \text { times }\end{array}$ & $\begin{array}{c}3 \\
4-5 \text { times }\end{array}$ & $\begin{array}{c}4 \\
6-10 \\
\text { times }\end{array}$ & More than 10 times \\
\hline Age 6 to 12 & $\begin{array}{c}0 \\
\text { Never }\end{array}$ & $\begin{array}{c}1 \\
\text { Once }\end{array}$ & $\begin{array}{c}2 \\
2-3 \text { times }\end{array}$ & $\begin{array}{c}3 \\
4-5 \text { times }\end{array}$ & $\begin{array}{c}4 \\
6-10 \\
\text { times }\end{array}$ & $\begin{array}{c}5 \\
\text { More than } 10 \text { times }\end{array}$ \\
\hline Age 13 to 18 & $\begin{array}{c}1 \\
\text { Never }\end{array}$ & $\begin{array}{c}1 \\
\text { Once }\end{array}$ & $\begin{array}{c}2 \\
2-3 \text { times }\end{array}$ & $\begin{array}{c}3 \\
4-5 \text { times }\end{array}$ & $\begin{array}{c}4 \\
6-10 \\
\text { times }\end{array}$ & More than 10 times \\
\hline Age 18+ & $\begin{array}{c}1 \\
\text { Never }\end{array}$ & Once & $\begin{array}{c}2 \\
2-3 \text { times }\end{array}$ & $\begin{array}{c}3 \\
4-5 \text { times } \\
\text { times }\end{array}$ & More than 10 times \\
\hline
\end{tabular}

\section{Did you ever experience the death or serious injury of a friend?}

\begin{tabular}{l|c|c|c|c|c|c}
\hline Age 0 to 5 & 0 & 1 & 2 & 3 & 4 & 5 \\
& Never & Once & $2-3$ times & $4-5$ times & & More than 10 times
\end{tabular}




\begin{tabular}{|c|c|c|c|c|c|c|}
\hline & & & & & $\begin{array}{c}6-10 \\
\text { times } \\
\end{array}$ & \\
\hline Age 6 to 12 & $\begin{array}{c}0 \\
\text { Never }\end{array}$ & $\begin{array}{c}1 \\
\text { Once }\end{array}$ & $\begin{array}{c}2 \\
2-3 \text { times }\end{array}$ & $\begin{array}{c}3 \\
4-5 \text { times }\end{array}$ & $\begin{array}{c}4 \\
6-10 \\
\text { times }\end{array}$ & $\begin{array}{c}5 \\
\text { More than } 10 \text { times }\end{array}$ \\
\hline Age 13 to 18 & $\begin{array}{c}0 \\
\text { Never }\end{array}$ & $\begin{array}{c}1 \\
\text { Once }\end{array}$ & $\begin{array}{c}2 \\
2-3 \text { times }\end{array}$ & $\begin{array}{c}3 \\
4-5 \text { times }\end{array}$ & $\begin{array}{c}4 \\
6-10 \\
\text { times }\end{array}$ & $\begin{array}{c}5 \\
\text { More than } 10 \text { times }\end{array}$ \\
\hline Age $18+$ & $\begin{array}{c}0 \\
\text { Never }\end{array}$ & $\begin{array}{c}1 \\
\text { Once }\end{array}$ & $\begin{array}{c}2 \\
2-3 \text { times }\end{array}$ & $\begin{array}{c}3 \\
4-5 \text { times }\end{array}$ & $\begin{array}{c}4 \\
6-10 \\
\text { times } \\
\end{array}$ & $\begin{array}{c}5 \\
\text { More than } 10 \text { times }\end{array}$ \\
\hline
\end{tabular}

\begin{tabular}{|c|c|c|c|c|c|c|}
\hline \multicolumn{7}{|c|}{ 8. Did you ever witness violence towards others, including family members? } \\
\hline Age 0 to 5 & $\begin{array}{c}0 \\
\text { Never }\end{array}$ & $\begin{array}{c}1 \\
\text { Once }\end{array}$ & $\begin{array}{c}2 \\
2-3 \text { times }\end{array}$ & $\begin{array}{c}3 \\
4-5 \text { times }\end{array}$ & $\begin{array}{c}4 \\
6-10 \\
\text { times }\end{array}$ & $\begin{array}{c}5 \\
\text { More than } 10 \text { times }\end{array}$ \\
\hline Age 6 to 12 & $\begin{array}{c}0 \\
\text { Never }\end{array}$ & $\begin{array}{c}1 \\
\text { Once }\end{array}$ & $\begin{array}{c}2 \\
2-3 \text { times }\end{array}$ & $\begin{array}{c}3 \\
4-5 \text { times }\end{array}$ & $\begin{array}{c}4 \\
6-10 \\
\text { times } \\
\end{array}$ & $\begin{array}{c}5 \\
\text { More than } 10 \text { times }\end{array}$ \\
\hline Age 13 to 18 & $\begin{array}{c}0 \\
\text { Never }\end{array}$ & $\begin{array}{c}1 \\
\text { Once }\end{array}$ & $\begin{array}{c}2 \\
2-3 \text { times }\end{array}$ & $\begin{array}{c}3 \\
4-5 \text { times }\end{array}$ & $\begin{array}{c}4 \\
6-10 \\
\text { times }\end{array}$ & $\begin{array}{c}5 \\
\text { More than } 10 \text { times }\end{array}$ \\
\hline Age $18+$ & $\begin{array}{c}0 \\
\text { Never }\end{array}$ & $\begin{array}{c}1 \\
\text { Once }\end{array}$ & $\begin{array}{c}2 \\
2-3 \text { times }\end{array}$ & $\begin{array}{c}3 \\
4-5 \text { times }\end{array}$ & $\begin{array}{c}4 \\
6-10 \\
\text { times }\end{array}$ & $\begin{array}{c}5 \\
\text { More than } 10 \text { times }\end{array}$ \\
\hline
\end{tabular}

\begin{tabular}{|c|c|c|c|c|c|c|}
\hline \multicolumn{7}{|c|}{$\begin{array}{l}\text { 9. Did anyone in your family ever suffer from mental or psychiatric illness or have a } \\
\text { "breakdown"? }\end{array}$} \\
\hline Age 0 to 5 & $\begin{array}{c}0 \\
\text { Never }\end{array}$ & $\begin{array}{c}1 \\
\text { Once }\end{array}$ & $\begin{array}{c}2 \\
2-3 \text { times }\end{array}$ & $\begin{array}{l}3 \\
4-5 \text { times }\end{array}$ & $\begin{array}{c}4 \\
6-10 \\
\text { times }\end{array}$ & $\begin{array}{c}5 \\
\text { More than } 10 \text { times }\end{array}$ \\
\hline Age 6 to 12 & $\begin{array}{c}0 \\
\text { Never }\end{array}$ & $\begin{array}{c}1 \\
\text { Once }\end{array}$ & $\begin{array}{l}2 \\
2-3 \text { times }\end{array}$ & $\begin{array}{l}3 \\
4-5 \text { times }\end{array}$ & $\begin{array}{c}4 \\
6-10 \\
\text { times } \\
\end{array}$ & $\begin{array}{c}5 \\
\text { More than } 10 \text { times }\end{array}$ \\
\hline Age 13 to 18 & $\begin{array}{c}0 \\
\text { Never }\end{array}$ & $\begin{array}{c}1 \\
\text { Once }\end{array}$ & $\begin{array}{c}2 \\
2-3 \text { times }\end{array}$ & $\begin{array}{c}3 \\
\text { 4-5 times }\end{array}$ & $\begin{array}{c} \\
6-10 \\
\text { times } \\
\end{array}$ & $\begin{array}{c}5 \\
\text { More than } 10 \text { times }\end{array}$ \\
\hline Age $18+$ & $\begin{array}{c}0 \\
\text { Never }\end{array}$ & $\begin{array}{c}1 \\
\text { Once }\end{array}$ & $\begin{array}{c}2 \\
2-3 \text { times }\end{array}$ & $\begin{array}{c}3 \\
4-5 \text { times }\end{array}$ & $\begin{array}{c}4 \\
6-10 \\
\text { times }\end{array}$ & $\begin{array}{c}5 \\
\text { More than } 10 \text { times }\end{array}$ \\
\hline
\end{tabular}

10. Did your parents or primary caretaker have a problem with alcoholism or drug abuse?
\begin{tabular}{|l|c|c|c|c|c|c}
\hline Age 0 to 5 & $\begin{array}{c}0 \\
\text { Never }\end{array}$ & $\begin{array}{c}1 \\
\text { Once }\end{array}$ & $\begin{array}{c}2 \\
2-3 \text { times }\end{array}$ & $\begin{array}{c}3 \\
4-5 \text { times }\end{array}$ & $\begin{array}{c}4 \\
6-10 \\
\text { times }\end{array}$ & $\begin{array}{c}5 \\
\text { More than } 10 \text { times }\end{array}$ \\
\hline Age 6 to 12 & $\begin{array}{c}0 \\
\text { Never }\end{array}$ & $\begin{array}{c}1 \\
\text { Once }\end{array}$ & $\begin{array}{c}2 \\
2-3 \text { times }\end{array}$ & $\begin{array}{c}3 \\
4-5 \text { times }\end{array}$ & $\begin{array}{c}4 \\
6-10 \\
\text { times }\end{array}$ & More than 10 times \\
\hline Age 13 to 18 & 0 & 1 & 2 & 3 & 4 & 5 \\
\hline
\end{tabular}




\begin{tabular}{|c|c|c|c|c|c|c|}
\hline & Never & Once & 2-3 times & 4-5 times & $\begin{array}{c}6-10 \\
\text { times } \\
\end{array}$ & More than 10 times \\
\hline Age $18+$ & $\begin{array}{c}0 \\
\text { Never }\end{array}$ & $\begin{array}{c}1 \\
\text { Once }\end{array}$ & $\begin{array}{c}2 \\
2-3 \text { times }\end{array}$ & $\begin{array}{c}3 \\
4-5 \text { times }\end{array}$ & $\begin{array}{c}4 \\
6-10 \\
\text { times }\end{array}$ & $\stackrel{5}{\text { More than } 10 \text { times }}$ \\
\hline \multicolumn{7}{|c|}{ 11. Did you ever see someone murdered? } \\
\hline Age 0 to 5 & $\begin{array}{c}0 \\
\text { Never }\end{array}$ & $\begin{array}{c}1 \\
\text { Once }\end{array}$ & $\begin{array}{c}2 \\
2-3 \text { times }\end{array}$ & $\begin{array}{c}3 \\
4-5 \text { times }\end{array}$ & $\begin{array}{c}4 \\
6-10 \\
\text { times } \\
\end{array}$ & $\begin{array}{c}5 \\
\text { More than } 10 \text { times }\end{array}$ \\
\hline Age 6 to 12 & $\begin{array}{c}0 \\
\text { Never }\end{array}$ & $\begin{array}{c}1 \\
\text { Once }\end{array}$ & $\begin{array}{c}2 \\
2-3 \text { times }\end{array}$ & $\begin{array}{c}3 \\
4-5 \text { times }\end{array}$ & $\begin{array}{c}4 \\
6-10 \\
\text { times } \\
\end{array}$ & $\begin{array}{c}5 \\
\text { More than } 10 \text { times }\end{array}$ \\
\hline Age 13 to 18 & $\begin{array}{c}0 \\
\text { Never }\end{array}$ & $\begin{array}{c}1 \\
\text { Once }\end{array}$ & $\begin{array}{c}2 \\
2-3 \text { times }\end{array}$ & $\begin{array}{c}3 \\
4-5 \text { times }\end{array}$ & $\begin{array}{c}4 \\
6-10 \\
\text { times }\end{array}$ & $\begin{array}{c}5 \\
\text { More than } 10 \text { times }\end{array}$ \\
\hline Age $18+$ & $\begin{array}{c}0 \\
\text { Never }\end{array}$ & $\begin{array}{c}1 \\
\text { Once }\end{array}$ & $\begin{array}{c}2 \\
2-3 \text { times }\end{array}$ & $\begin{array}{c}3 \\
4-5 \text { times }\end{array}$ & $\begin{array}{c}4 \\
6-10 \\
\text { times }\end{array}$ & $\begin{array}{c}5 \\
\text { More than } 10 \text { times }\end{array}$ \\
\hline
\end{tabular}

\section{Part 2: Physical Punishment}

\section{Were you ever slapped in the face with an open hand?}

\begin{tabular}{|c|c|c|c|c|c|c|}
\hline Age 0 to 5 & $\begin{array}{c}0 \\
\text { Never }\end{array}$ & $\begin{array}{c}1 \\
\text { Once }\end{array}$ & $\begin{array}{c}2 \\
2-3 \text { times }\end{array}$ & $\begin{array}{c}3 \\
4-5 \text { times }\end{array}$ & $\begin{array}{c} \\
6-10 \\
\text { times }\end{array}$ & $\begin{array}{c}5 \\
\text { More than } 10 \text { times }\end{array}$ \\
\hline Age 6 to 12 & $\begin{array}{c}0 \\
\text { Never }\end{array}$ & $\begin{array}{c}1 \\
\text { Once }\end{array}$ & $\begin{array}{c}2 \\
2-3 \text { times }\end{array}$ & $\begin{array}{c}3 \\
4-5 \text { times }\end{array}$ & $\begin{array}{c} \\
6-10 \\
\text { times } \\
\end{array}$ & $\begin{array}{c}5 \\
\text { More than } 10 \text { times }\end{array}$ \\
\hline Age 13 to 18 & $\begin{array}{c}0 \\
\text { Never }\end{array}$ & $\begin{array}{c}1 \\
\text { Once }\end{array}$ & $\begin{array}{c}2 \\
2-3 \text { times }\end{array}$ & $\begin{array}{c}3 \\
4-5 \text { times }\end{array}$ & $\begin{array}{c}4 \\
6-10 \\
\text { times } \\
\end{array}$ & $\begin{array}{c}5 \\
\text { More than } 10 \text { times }\end{array}$ \\
\hline Age $18+$ & $\begin{array}{c}0 \\
\text { Never }\end{array}$ & $\begin{array}{c}1 \\
\text { Once }\end{array}$ & $\begin{array}{c}2 \\
2-3 \text { times }\end{array}$ & $\begin{array}{c}3 \\
4-5 \text { times }\end{array}$ & $\begin{array}{c}4 \\
6-10 \\
\text { times }\end{array}$ & $\begin{array}{c}5 \\
\text { More than } 10 \text { times }\end{array}$ \\
\hline
\end{tabular}

2. Were you ever burned with hot water, a cigarette or something else?

\begin{tabular}{l|c|c|c|c|c|c}
\hline Age 0 to 5 & $\begin{array}{c}0 \\
\text { Never }\end{array}$ & $\begin{array}{c}1 \\
\text { Once }\end{array}$ & $\begin{array}{c}2 \\
2-3 \text { times }\end{array}$ & $\begin{array}{c}3 \\
4-5 \text { times }\end{array}$ & $\begin{array}{c}4 \\
6-10 \\
\text { times }\end{array}$ & More than 10 times \\
\hline Age 6 to 12 & $\begin{array}{c}0 \\
\text { Never }\end{array}$ & $\begin{array}{c}1 \\
\text { Once }\end{array}$ & $\begin{array}{c}2 \\
2-3 \text { times }\end{array}$ & $\begin{array}{c}3 \\
4-5 \text { times }\end{array}$ & $\begin{array}{c}4 \\
6-10 \\
\text { times }\end{array}$ & More than 10 times \\
\hline Age 13 to 18 & $\begin{array}{c}0 \\
\text { Never }\end{array}$ & $\begin{array}{c}1 \\
\text { Once }\end{array}$ & $\begin{array}{c}2 \\
2-3 \text { times }\end{array}$ & $\begin{array}{c}3 \\
4-5 \text { times }\end{array}$ & $\begin{array}{c}4 \\
6-10 \\
\text { times }\end{array}$ & More than 10 times
\end{tabular}




\begin{tabular}{|c|c|c|c|c|c|c|}
\hline Age $18+$ & $\begin{array}{c}0 \\
\text { Never }\end{array}$ & $\begin{array}{c}1 \\
\text { Once }\end{array}$ & $\begin{array}{c}2 \\
2-3 \text { times }\end{array}$ & $\begin{array}{c}3 \\
4-5 \text { times }\end{array}$ & $\begin{array}{c}4 \\
6-10 \\
\text { times }\end{array}$ & $\begin{array}{c}5 \\
\text { More than } 10 \text { times }\end{array}$ \\
\hline \multicolumn{7}{|c|}{ 3.Were you ever punched or kicked? } \\
\hline Age 0 to 5 & $\begin{array}{c}0 \\
\text { Never }\end{array}$ & $\begin{array}{c}1 \\
\text { Once }\end{array}$ & $\begin{array}{c}2 \\
2-3 \text { times }\end{array}$ & $\begin{array}{c}3 \\
4-5 \text { times }\end{array}$ & $\begin{array}{c} \\
6-10 \\
\text { times }\end{array}$ & $\begin{array}{c}5 \\
\text { More than } 10 \text { times }\end{array}$ \\
\hline Age 6 to 12 & $\begin{array}{c}0 \\
\text { Never }\end{array}$ & $\begin{array}{c}1 \\
\text { Once }\end{array}$ & $\begin{array}{c}2 \\
2-3 \text { times }\end{array}$ & $\begin{array}{c}3 \\
4-5 \text { times }\end{array}$ & $\begin{array}{c}4 \\
6-10 \\
\text { times } \\
\end{array}$ & $\begin{array}{c}5 \\
\text { More than } 10 \text { times }\end{array}$ \\
\hline Age 13 to 18 & $\begin{array}{c}0 \\
\text { Never }\end{array}$ & $\begin{array}{c}1 \\
\text { Once }\end{array}$ & $\begin{array}{c}2 \\
2-3 \text { times }\end{array}$ & $\begin{array}{c}3 \\
4-5 \text { times }\end{array}$ & $\begin{array}{c}4 \\
6-10 \\
\text { times }\end{array}$ & $\begin{array}{c}5 \\
\text { More than } 10 \text { times }\end{array}$ \\
\hline Age $18+$ & $\begin{array}{c}0 \\
\text { Never }\end{array}$ & $\begin{array}{c}1 \\
\text { Once }\end{array}$ & $\begin{array}{c}2 \\
2-3 \text { times }\end{array}$ & $\begin{array}{c}3 \\
4-5 \text { times }\end{array}$ & $\begin{array}{c}4 \\
6-10 \\
\text { times } \\
\end{array}$ & $\begin{array}{c}5 \\
\text { More than } 10 \text { times }\end{array}$ \\
\hline \multicolumn{7}{|c|}{ 4. Were you ever hit with an object that was thrown at you? } \\
\hline Age 0 to 5 & $\begin{array}{c}0 \\
\text { Never }\end{array}$ & $\begin{array}{c}1 \\
\text { Once }\end{array}$ & $\begin{array}{c}2 \\
2-3 \text { times }\end{array}$ & $\begin{array}{c}3 \\
4-5 \text { times }\end{array}$ & $\begin{array}{c}4 \\
6-10 \\
\text { times } \\
\end{array}$ & $\begin{array}{c}5 \\
\text { More than } 10 \text { times }\end{array}$ \\
\hline Age 6 to 12 & $\begin{array}{c}0 \\
\text { Never }\end{array}$ & $\begin{array}{c}1 \\
\text { Once }\end{array}$ & $\begin{array}{c}2 \\
2-3 \text { times }\end{array}$ & $\begin{array}{c}3 \\
4-5 \text { times }\end{array}$ & $\begin{array}{c}4 \\
6-10 \\
\text { times } \\
\end{array}$ & $\begin{array}{c}5 \\
\text { More than } 10 \text { times }\end{array}$ \\
\hline Age 13 to 18 & $\begin{array}{c}0 \\
\text { Never }\end{array}$ & $\begin{array}{c}1 \\
\text { Once }\end{array}$ & $\begin{array}{c}2 \\
2-3 \text { times }\end{array}$ & $\begin{array}{c}3 \\
4-5 \text { times }\end{array}$ & $\begin{array}{c}4 \\
6-10 \\
\text { times } \\
\end{array}$ & $\begin{array}{c}5 \\
\text { More than } 10 \text { times }\end{array}$ \\
\hline Age $18+$ & $\begin{array}{c}0 \\
\text { Never }\end{array}$ & $\begin{array}{c}1 \\
\text { Once }\end{array}$ & $\begin{array}{c}2 \\
2-3 \text { times }\end{array}$ & $\begin{array}{c}3 \\
4-5 \text { times }\end{array}$ & $\begin{array}{c}4 \\
6-10 \\
\text { times }\end{array}$ & $\begin{array}{c}5 \\
\text { More than } 10 \text { times }\end{array}$ \\
\hline \multicolumn{7}{|c|}{ 5.Were you ever pushed or shoved? } \\
\hline Age 0 to 5 & $\begin{array}{c}0 \\
\text { Never }\end{array}$ & $\begin{array}{c}1 \\
\text { Once }\end{array}$ & $\begin{array}{c}2 \\
2-3 \text { times }\end{array}$ & $\begin{array}{c}3 \\
4-5 \text { times }\end{array}$ & $\begin{array}{c}4 \\
6-10 \\
\text { times } \\
\end{array}$ & $\begin{array}{c}5 \\
\text { More than } 10 \text { times }\end{array}$ \\
\hline Age 6 to 12 & $\begin{array}{c}0 \\
\text { Never }\end{array}$ & $\begin{array}{c}1 \\
\text { Once }\end{array}$ & $\begin{array}{c}2 \\
2-3 \text { times }\end{array}$ & $\begin{array}{c}3 \\
4-5 \text { times }\end{array}$ & $\begin{array}{c}4 \\
6-10 \\
\text { times }\end{array}$ & $\begin{array}{c}5 \\
\text { More than } 10 \text { times }\end{array}$ \\
\hline Age 13 to 18 & $\begin{array}{c}0 \\
\text { Never }\end{array}$ & $\begin{array}{c}1 \\
\text { Once }\end{array}$ & $\begin{array}{c}2 \\
2-3 \text { times }\end{array}$ & $\begin{array}{c}3 \\
4-5 \text { times }\end{array}$ & $\begin{array}{c}4 \\
6-10 \\
\text { times }\end{array}$ & $\begin{array}{c}5 \\
\text { More than } 10 \text { times }\end{array}$ \\
\hline Age $18+$ & $\begin{array}{c}0 \\
\text { Never }\end{array}$ & $\begin{array}{c}1 \\
\text { Once }\end{array}$ & $\begin{array}{c}2 \\
2-3 \text { times }\end{array}$ & $\begin{array}{c}3 \\
4-5 \text { times }\end{array}$ & $\begin{array}{c}4 \\
6-10 \\
\text { times }\end{array}$ & $\begin{array}{c}5 \\
\text { More than } 10 \text { times }\end{array}$ \\
\hline
\end{tabular}


Part 3: Emotional Abuse

1. Were you often put down or ridiculed?

\begin{tabular}{|c|c|c|c|c|c|c|}
\hline Age 0 to 5 & $\begin{array}{c}0 \\
\text { Never }\end{array}$ & $\begin{array}{c}1 \\
\text { Once }\end{array}$ & $\begin{array}{c}2 \\
2-3 \text { times }\end{array}$ & $\begin{array}{c}3 \\
4-5 \text { times }\end{array}$ & $\begin{array}{c}4 \\
6-10 \\
\text { times }\end{array}$ & $\begin{array}{c}5 \\
\text { More than } 10 \text { times }\end{array}$ \\
\hline Age 6 to 12 & $\begin{array}{c}0 \\
\text { Never }\end{array}$ & $\begin{array}{c}1 \\
\text { Once }\end{array}$ & $\begin{array}{c}2 \\
2-3 \text { times }\end{array}$ & $\begin{array}{c}3 \\
4-5 \text { times }\end{array}$ & $\begin{array}{c}4 \\
6-10 \\
\text { times }\end{array}$ & $\begin{array}{c}5 \\
\text { More than } 10 \text { times }\end{array}$ \\
\hline Age 13 to 18 & $\begin{array}{c}0 \\
\text { Never }\end{array}$ & $\begin{array}{c}1 \\
\text { Once }\end{array}$ & $\begin{array}{c}2 \\
2-3 \text { times }\end{array}$ & $\begin{array}{c}3 \\
4-5 \text { times }\end{array}$ & $\begin{array}{c}4 \\
6-10 \\
\text { times }\end{array}$ & $\begin{array}{c}5 \\
\text { More than } 10 \text { times }\end{array}$ \\
\hline Age $18+$ & $\begin{array}{c}0 \\
\text { Never }\end{array}$ & $\begin{array}{c}1 \\
\text { Once }\end{array}$ & $\begin{array}{c}2 \\
2-3 \text { times }\end{array}$ & $\begin{array}{c}3 \\
4-5 \text { times }\end{array}$ & $\begin{array}{c}4 \\
6-10 \\
\text { times }\end{array}$ & $\begin{array}{c}5 \\
\text { More than } 10 \text { times }\end{array}$ \\
\hline
\end{tabular}

2. Were you often ignored or made to feel that you didn't count?

\begin{tabular}{|c|c|c|c|c|c|c|}
\hline Age 0 to 5 & $\begin{array}{c}0 \\
\text { Never }\end{array}$ & $\begin{array}{c}1 \\
\text { Once }\end{array}$ & $\begin{array}{c}2 \\
2-3 \text { times }\end{array}$ & $\begin{array}{c}3 \\
4-5 \text { times }\end{array}$ & $\begin{array}{c}4 \\
6-10 \\
\text { times }\end{array}$ & $\begin{array}{c}5 \\
\text { More than } 10 \text { times }\end{array}$ \\
\hline Age 6 to 12 & $\begin{array}{c}0 \\
\text { Never }\end{array}$ & $\begin{array}{c}1 \\
\text { Once }\end{array}$ & $\begin{array}{c}2 \\
2-3 \text { times }\end{array}$ & $\begin{array}{c}3 \\
4-5 \text { times }\end{array}$ & $\begin{array}{c}4 \\
6-10 \\
\text { times } \\
\end{array}$ & $\begin{array}{c}5 \\
\text { More than } 10 \text { times }\end{array}$ \\
\hline Age 13 to 18 & $\begin{array}{c}0 \\
\text { Never }\end{array}$ & $\begin{array}{c}1 \\
\text { Once }\end{array}$ & $\begin{array}{c}2 \\
2-3 \text { times }\end{array}$ & $\begin{array}{c}3 \\
4-5 \text { times }\end{array}$ & $\begin{array}{c} \\
6-10 \\
\text { times }\end{array}$ & $\begin{array}{c}5 \\
\text { More than } 10 \text { times }\end{array}$ \\
\hline Age $18+$ & $\begin{array}{c}0 \\
\text { Never }\end{array}$ & $\begin{array}{c}1 \\
\text { Once }\end{array}$ & $\begin{array}{c}2 \\
2-3 \text { times }\end{array}$ & $\begin{array}{c}3 \\
4-5 \text { times }\end{array}$ & $\begin{array}{c}4 \\
6-10 \\
\text { times } \\
\end{array}$ & $\begin{array}{c}5 \\
\text { More than } 10 \text { times }\end{array}$ \\
\hline
\end{tabular}

\begin{tabular}{|c|c|c|c|c|c|c|}
\hline \multicolumn{7}{|c|}{ 3. Were you often told you were no good? } \\
\hline Age 0 to 5 & $\begin{array}{c}0 \\
\text { Never }\end{array}$ & $\begin{array}{c}1 \\
\text { Once }\end{array}$ & $\begin{array}{c}2 \\
2-3 \text { times }\end{array}$ & $\begin{array}{c}3 \\
4-5 \text { times }\end{array}$ & $\begin{array}{c}4 \\
6-10 \\
\text { times }\end{array}$ & $\begin{array}{c}5 \\
\text { More than } 10 \text { times }\end{array}$ \\
\hline Age 6 to 12 & $\begin{array}{c}0 \\
\text { Never }\end{array}$ & $\begin{array}{c}1 \\
\text { Once }\end{array}$ & $\begin{array}{c}2 \\
2-3 \text { times }\end{array}$ & $\begin{array}{c}3 \\
4-5 \text { times }\end{array}$ & $\begin{array}{c}4 \\
6-10 \\
\text { times }\end{array}$ & $\begin{array}{c}5 \\
\text { More than } 10 \text { times }\end{array}$ \\
\hline Age 13 to 18 & $\begin{array}{c}0 \\
\text { Never }\end{array}$ & $\begin{array}{c}1 \\
\text { Once }\end{array}$ & $\begin{array}{c}2 \\
2-3 \text { times }\end{array}$ & $\begin{array}{c}3 \\
4-5 \text { times }\end{array}$ & $\begin{array}{c} \\
6-10 \\
\text { times }\end{array}$ & $\begin{array}{c}5 \\
\text { More than } 10 \text { times }\end{array}$ \\
\hline Age $18+$ & $\begin{array}{c}0 \\
\text { Never }\end{array}$ & $\begin{array}{c}1 \\
\text { Once }\end{array}$ & $\begin{array}{c}2 \\
2-3 \text { times }\end{array}$ & $\begin{array}{c}3 \\
4-5 \text { times }\end{array}$ & $\begin{array}{c} \\
6-10 \\
\text { times }\end{array}$ & $\begin{array}{c}5 \\
\text { More than } 10 \text { times }\end{array}$ \\
\hline
\end{tabular}

4. Most of the time were you treated in a cold, uncaring way or made to feel like you were not loved?

\begin{tabular}{l|c|c|c|c|c|c}
\hline Age 0 to 5 & 0 & 1 & 2 & 3 & 4 & 5 \\
Never & Once & $2-3$ times & $\begin{array}{c}4-5 \text { times } \\
6-10 \\
\text { times }\end{array}$ & More than 10 times
\end{tabular}




\begin{tabular}{l|c|c|c|c|c|c} 
Age 6 to 12 & $\begin{array}{c}0 \\
\text { Never }\end{array}$ & $\begin{array}{c}1 \\
\text { Once }\end{array}$ & $\begin{array}{c}2 \\
2-3 \text { times }\end{array}$ & $\begin{array}{c}3 \\
4-5 \text { times }\end{array}$ & $\begin{array}{c}4 \\
6-10 \\
\text { times }\end{array}$ & $\begin{array}{c}5 \\
\text { Agore than } 10 \text { times }\end{array}$ \\
\hline Age 13 to 18 & $\begin{array}{c}0 \\
\text { Never }\end{array}$ & $\begin{array}{c}1 \\
\text { Once }\end{array}$ & $\begin{array}{c}2 \\
2-3 \text { times }\end{array}$ & $\begin{array}{c}3 \\
4-5 \text { times }\end{array}$ & $\begin{array}{c}4 \\
6-10 \\
\text { times }\end{array}$ & More than 10 times \\
\hline Age 18+ & $\begin{array}{c}1 \\
\text { Never }\end{array}$ & $\begin{array}{c}1 \\
\text { Once }\end{array}$ & $\begin{array}{c}2 \\
2-3 \text { times }\end{array}$ & $\begin{array}{c}3 \\
4-5 \text { times }\end{array}$ & $\begin{array}{c}4 \\
6-10 \\
\text { times }\end{array}$ & More than 10 times \\
\hline
\end{tabular}

\section{Did your parents or caretakers often fail to understand you or your needs?}

\begin{tabular}{|c|c|c|c|c|c|c|}
\hline Age 0 to 5 & $\begin{array}{c}0 \\
\text { Never }\end{array}$ & $\begin{array}{c}1 \\
\text { Once }\end{array}$ & $\begin{array}{c}2 \\
2-3 \text { times }\end{array}$ & $\begin{array}{c}3 \\
4-5 \text { times }\end{array}$ & $\begin{array}{c} \\
6-10 \\
\text { times }\end{array}$ & $\begin{array}{c}5 \\
\text { More than } 10 \text { times }\end{array}$ \\
\hline Age 6 to 12 & $\begin{array}{c}0 \\
\text { Never }\end{array}$ & $\begin{array}{c}1 \\
\text { Once }\end{array}$ & $\begin{array}{c}2 \\
2-3 \text { times }\end{array}$ & $\begin{array}{c}3 \\
4-5 \text { times }\end{array}$ & $\begin{array}{c}4 \\
6-10 \\
\text { times } \\
\end{array}$ & $\begin{array}{c}5 \\
\text { More than } 10 \text { times }\end{array}$ \\
\hline Age 13 to 18 & $\begin{array}{c}0 \\
\text { Never }\end{array}$ & $\begin{array}{c}1 \\
\text { Once }\end{array}$ & $\begin{array}{c}2 \\
2-3 \text { times }\end{array}$ & $\begin{array}{c}3 \\
4-5 \text { times }\end{array}$ & $\begin{array}{c}4 \\
6-10 \\
\text { times } \\
\end{array}$ & $\begin{array}{c}5 \\
\text { More than } 10 \text { times }\end{array}$ \\
\hline Age $18+$ & $\begin{array}{c}0 \\
\text { Never }\end{array}$ & $\begin{array}{c}1 \\
\text { Once }\end{array}$ & $\begin{array}{c}2 \\
2-3 \text { times }\end{array}$ & $\begin{array}{c}3 \\
4-5 \text { times }\end{array}$ & $\begin{array}{c}4 \\
6-10 \\
\text { times }\end{array}$ & $\begin{array}{c}5 \\
\text { More than } 10 \text { times }\end{array}$ \\
\hline
\end{tabular}

\section{Part 4: Sexual Events}

1. Were you ever touched in an intimate or private part of your body (e.g. breast, thighs, genitals) in a way that surprised you or made you feel uncomfortable?

\begin{tabular}{|c|c|c|c|c|c|c|}
\hline Age 0 to 5 & $\begin{array}{c}0 \\
\text { Never }\end{array}$ & $\begin{array}{c}1 \\
\text { Once }\end{array}$ & $\begin{array}{c}2 \\
2-3 \text { times }\end{array}$ & $\begin{array}{c}3 \\
4-5 \text { times }\end{array}$ & $\begin{array}{c}4 \\
6-10 \\
\text { times }\end{array}$ & $\begin{array}{c}5 \\
\text { More than } 10 \text { times }\end{array}$ \\
\hline Age 6 to 12 & $\begin{array}{c}0 \\
\text { Never }\end{array}$ & $\begin{array}{c}1 \\
\text { Once }\end{array}$ & $\begin{array}{c}2 \\
2-3 \text { times }\end{array}$ & $\begin{array}{c}3 \\
4-5 \text { times }\end{array}$ & $\begin{array}{c}4 \\
6-10 \\
\text { times } \\
\end{array}$ & $\begin{array}{c}5 \\
\text { More than } 10 \text { times }\end{array}$ \\
\hline Age 13 to 18 & $\begin{array}{c}0 \\
\text { Never }\end{array}$ & $\begin{array}{c}1 \\
\text { Once }\end{array}$ & $\begin{array}{c}2 \\
2-3 \text { times }\end{array}$ & $\begin{array}{c}3 \\
4-5 \text { times }\end{array}$ & $\begin{array}{c}4 \\
6-10 \\
\text { times } \\
\end{array}$ & $\begin{array}{c}5 \\
\text { More than } 10 \text { times }\end{array}$ \\
\hline Age $18+$ & $\begin{array}{c}0 \\
\text { Never }\end{array}$ & $\begin{array}{c}1 \\
\text { Once }\end{array}$ & $\begin{array}{c}2 \\
2-3 \text { times }\end{array}$ & $\begin{array}{c}3 \\
4-5 \text { times }\end{array}$ & $\begin{array}{c}4 \\
6-10 \\
\text { times } \\
\end{array}$ & $\begin{array}{c}5 \\
\text { More than } 10 \text { times }\end{array}$ \\
\hline
\end{tabular}

\section{Did you ever experience someone rubbing their genitals against you?}

\begin{tabular}{l|c|c|c|c|c|c}
\hline Age 0 to 5 & $\begin{array}{c}0 \\
\text { Never }\end{array}$ & $\begin{array}{c}1 \\
\text { Once }\end{array}$ & $\begin{array}{c}2 \\
2-3 \text { times }\end{array}$ & $\begin{array}{c}3 \\
4-5 \text { times }\end{array}$ & $\begin{array}{c}4 \\
6-10 \\
\text { times }\end{array}$ & $\begin{array}{c}5 \\
\text { More than } 10 \text { times }\end{array}$ \\
\hline Age 6 to 12 & $\begin{array}{c}0 \\
\text { Never }\end{array}$ & $\begin{array}{c}1 \\
\text { Once }\end{array}$ & $\begin{array}{c}2 \\
2-3 \text { times }\end{array}$ & $\begin{array}{c}3 \\
4-5 \text { times }\end{array}$ & $\begin{array}{c}4 \\
6-10 \\
\text { times }\end{array}$ & More than 10 times \\
\hline Age 13 to 18 & 0 & 1 & 2 & 3 & 4 & 5
\end{tabular}




\begin{tabular}{l|c|c|c|c|c|c} 
& Never & Once & $2-3$ times & $4-5$ times & $\begin{array}{c}6-10 \\
\text { times }\end{array}$ & More than 10 times \\
\hline Age 18+ & $\begin{array}{c}0 \\
\text { Never }\end{array}$ & $\begin{array}{c}1 \\
\text { Once }\end{array}$ & $\begin{array}{c}2 \\
2-3 \text { times }\end{array}$ & $\begin{array}{c}3 \\
4-5 \text { times }\end{array}$ & $\begin{array}{c}4 \\
6-10 \\
\text { times }\end{array}$ & More than 10 times \\
\hline
\end{tabular}

\begin{tabular}{|c|c|c|c|c|c|c|}
\hline \multicolumn{7}{|c|}{$\begin{array}{l}\text { 3. Were you ever forced or coerced to touch another person in an intimate or private part of their } \\
\text { body? }\end{array}$} \\
\hline Age 0 to 5 & $\begin{array}{c}0 \\
\text { Never }\end{array}$ & $\begin{array}{c}1 \\
\text { Once }\end{array}$ & $\begin{array}{c}2 \\
2-3 \text { times }\end{array}$ & $\begin{array}{c}3 \\
4-5 \text { times }\end{array}$ & $\begin{array}{c} \\
6-10 \\
\text { times }\end{array}$ & $\begin{array}{c}5 \\
\text { More than } 10 \text { times }\end{array}$ \\
\hline Age 6 to 12 & $\begin{array}{c}0 \\
\text { Never }\end{array}$ & $\begin{array}{c}1 \\
\text { Once }\end{array}$ & $\begin{array}{c}2 \\
2-3 \text { times }\end{array}$ & $\begin{array}{c}3 \\
4-5 \text { times }\end{array}$ & $\begin{array}{c} \\
6-10 \\
\text { times }\end{array}$ & $\begin{array}{c}5 \\
\text { More than } 10 \text { times }\end{array}$ \\
\hline Age 13 to 18 & $\begin{array}{c}0 \\
\text { Never }\end{array}$ & $\begin{array}{c}1 \\
\text { Once }\end{array}$ & $\begin{array}{c}2 \\
2-3 \text { times }\end{array}$ & $\begin{array}{c}3 \\
4-5 \text { times }\end{array}$ & $\begin{array}{c}4 \\
6-10 \\
\text { times }\end{array}$ & $\begin{array}{c}5 \\
\text { More than } 10 \text { times }\end{array}$ \\
\hline Age $18+$ & $\begin{array}{c}0 \\
\text { Never }\end{array}$ & $\begin{array}{c}1 \\
\text { Once }\end{array}$ & $\begin{array}{c}2 \\
2-3 \text { times }\end{array}$ & $\begin{array}{c}3 \\
4-5 \text { times }\end{array}$ & $\begin{array}{c}4 \\
6-10 \\
\text { times }\end{array}$ & $\begin{array}{c}5 \\
\text { More than } 10 \text { times }\end{array}$ \\
\hline
\end{tabular}

\begin{tabular}{|c|c|c|c|c|c|c|}
\hline \multicolumn{7}{|c|}{ 4. Did anyone ever have genital sex with you against your will? } \\
\hline Age 0 to 5 & $\begin{array}{c}0 \\
\text { Never }\end{array}$ & $\begin{array}{c}1 \\
\text { Once }\end{array}$ & $\begin{array}{c}2 \\
2-3 \text { times }\end{array}$ & $\begin{array}{c}3 \\
4-5 \text { times }\end{array}$ & $\begin{array}{c}4 \\
6-10 \\
\text { times }\end{array}$ & $\begin{array}{c}5 \\
\text { More than } 10 \text { times }\end{array}$ \\
\hline Age 6 to 12 & $\begin{array}{c}0 \\
\text { Never }\end{array}$ & $\begin{array}{c}1 \\
\text { Once }\end{array}$ & $\begin{array}{c}2 \\
2-3 \text { times }\end{array}$ & $\begin{array}{c}3 \\
4-5 \text { times }\end{array}$ & $\begin{array}{c}4 \\
6-10 \\
\text { times }\end{array}$ & $\begin{array}{c}5 \\
\text { More than } 10 \text { times }\end{array}$ \\
\hline Age 13 to 18 & $\begin{array}{c}0 \\
\text { Never }\end{array}$ & $\begin{array}{c}1 \\
\text { Once }\end{array}$ & $\begin{array}{c}2 \\
2-3 \text { times }\end{array}$ & $\begin{array}{c}3 \\
4-5 \text { times }\end{array}$ & $\begin{array}{c}4 \\
6-10 \\
\text { times }\end{array}$ & $\begin{array}{c}5 \\
\text { More than } 10 \text { times }\end{array}$ \\
\hline Age $18+$ & $\begin{array}{c}0 \\
\text { Never }\end{array}$ & $\begin{array}{c}1 \\
\text { Once }\end{array}$ & $\begin{array}{c}2 \\
2-3 \text { times }\end{array}$ & $\begin{array}{c}3 \\
4-5 \text { times }\end{array}$ & $\begin{array}{c}4 \\
6-10 \\
\text { times }\end{array}$ & $\begin{array}{c}5 \\
\text { More than } 10 \text { times }\end{array}$ \\
\hline
\end{tabular}

5. Were you ever forced or coerced to perform oral sex on someone against your will?

\begin{tabular}{|c|c|c|c|c|c|c|}
\hline Age 0 to 5 & $\begin{array}{c}0 \\
\text { Never }\end{array}$ & $\begin{array}{c}1 \\
\text { Once }\end{array}$ & $\begin{array}{c}2 \\
2-3 \text { times }\end{array}$ & $\begin{array}{c}3 \\
4-5 \text { times }\end{array}$ & $\begin{array}{c}4 \\
6-10 \\
\text { times }\end{array}$ & $\begin{array}{c}5 \\
\text { More than } 10 \text { times }\end{array}$ \\
\hline Age 6 to 12 & $\begin{array}{c}0 \\
\text { Never }\end{array}$ & $\begin{array}{c}1 \\
\text { Once }\end{array}$ & $\begin{array}{c}2 \\
2-3 \text { times }\end{array}$ & $\begin{array}{c}3 \\
4-5 \text { times }\end{array}$ & $\begin{array}{c}4 \\
6-10 \\
\text { times }\end{array}$ & $\begin{array}{c}5 \\
\text { More than } 10 \text { times }\end{array}$ \\
\hline Age 13 to 18 & $\begin{array}{c}0 \\
\text { Never }\end{array}$ & $\begin{array}{c}1 \\
\text { Once }\end{array}$ & $\begin{array}{c}2 \\
2-3 \text { times }\end{array}$ & $\begin{array}{c}3 \\
4-5 \text { times }\end{array}$ & $\begin{array}{c}4 \\
6-10 \\
\text { times }\end{array}$ & $\begin{array}{c}5 \\
\text { More than } 10 \text { times }\end{array}$ \\
\hline Age $18+$ & $\begin{array}{c}0 \\
\text { Never }\end{array}$ & $\begin{array}{c}1 \\
\text { Once }\end{array}$ & $\begin{array}{c}2 \\
2-3 \text { times }\end{array}$ & $\begin{array}{c}3 \\
4-5 \text { times }\end{array}$ & $\begin{array}{c}4 \\
6-10 \\
\text { times }\end{array}$ & $\begin{array}{c}5 \\
\text { More than } 10 \text { times }\end{array}$ \\
\hline
\end{tabular}

6. Were you ever forced or coerced to kiss someone in a sexual rather than an affectionate way? 


\begin{tabular}{|c|c|c|c|c|c|c|}
\hline Age 0 to 5 & $\begin{array}{c}0 \\
\text { Never }\end{array}$ & $\begin{array}{c}1 \\
\text { Once }\end{array}$ & $\begin{array}{c}2 \\
2-3 \text { times }\end{array}$ & $\begin{array}{c}3 \\
4-5 \text { times }\end{array}$ & $\begin{array}{c}4 \\
6-10 \\
\text { times }\end{array}$ & $\begin{array}{c}5 \\
\text { More than } 10 \text { times }\end{array}$ \\
\hline Age 6 to 12 & $\begin{array}{c}0 \\
\text { Never }\end{array}$ & $\begin{array}{c}1 \\
\text { Once }\end{array}$ & $\begin{array}{c}2 \\
2-3 \text { times }\end{array}$ & $\begin{array}{c}3 \\
4-5 \text { times }\end{array}$ & $\begin{array}{c}4 \\
6-10 \\
\text { times }\end{array}$ & $\begin{array}{c}5 \\
\text { More than } 10 \text { times }\end{array}$ \\
\hline Age 13 to 18 & $\begin{array}{c}0 \\
\text { Never }\end{array}$ & $\begin{array}{c}1 \\
\text { Once }\end{array}$ & $\begin{array}{c}2 \\
2-3 \text { times }\end{array}$ & $\begin{array}{c}3 \\
4-5 \text { times }\end{array}$ & $\begin{array}{c}4 \\
6-10 \\
\text { times }\end{array}$ & $\begin{array}{c}5 \\
\text { More than } 10 \text { times }\end{array}$ \\
\hline Age $18+$ & $\begin{array}{c}0 \\
\text { Never }\end{array}$ & $\begin{array}{c}1 \\
\text { Once }\end{array}$ & $\begin{array}{c}2 \\
2-3 \text { times }\end{array}$ & $\begin{array}{c}3 \\
4-5 \text { times }\end{array}$ & $\begin{array}{c}4 \\
6-10 \\
\text { times }\end{array}$ & $\begin{array}{c}5 \\
\text { More than } 10 \text { times }\end{array}$ \\
\hline
\end{tabular}




\section{State-Trait Anxiety Inventory (STAI)}

DIRECTIONS: A number of statements which people have used to describe themselves are given below. Read each statement and then circle the number to the right of the statement to indicate how you feel right now, that is, at this moment. There are no right or wrong answers. Do not spend too much time on any one statement but give the answer which seems to describe your present feelings best.

Not at all

1

Somewhat so

2

1. I feel calm

2. I feel secure

3. I am tense

4. I feel strained

5. I feel at ease

6. I feel upset

7. I am presently worrying over possible. misfortunes.

8. I feel satisfied...

9. I feel frightened

10. I feel comfortable

11. I feel self-confident

12. I feel nervous.

13. I am jittery

14. I feel indecisive

15. I am relaxed

16. I feel content

17. I am worried

18. I feel confused

19. I feel steady

20. I feel pleasant
Moderately so

3
Very much

4

4

4

4

4

4

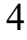

4

4

4

4

4

4

4

4

4

4

4

4

4

4 


\section{Ruminative Responses Scale (RRS)}

Using the scale provided choose the corresponding numerical representation that best describes your own general attitude and feelings when faced with problems. Please fill in the appropriate number in the space provided next to each question.

1

Almost

Never
2

Sometimes
3

Often
4

Almost

Always

Please indicate what you generally do, not what you think you should do.

\section{When you encounter problems, you:}

1. think about how alone you feel

2. think "I won't be able to do my job if I don't snap out of this."

3. think about your feelings of fatigue and achiness

4. think about how hard it is to concentrate

5. think "What am I doing to deserve this?"

6. think about how passive and unmotivated you feel

7. analyze recent events to try to understand why you are depressed

8. think about how you don't seem to feel anything anymore

9. think "Why can't I get going?"

10. think "Why do I always react this way?"

11. go away by yourself and think about why you feel this way

12. write down what you are thinking and analyze it

13. think about a recent situation, wishing it had gone better

14. think "I won't be able to concentrate if I keep feeling this way."

15. think "Why do I have problems other people don't have?"

16. think "Why can't I handle things better?" 
17. think about how sad you feel

18. think about all your shortcomings, failings, faults, mistakes

19. think about how you don't feel up to doing anything

20. analyze your personality to try to understand why you are depressed

21. go someplace alone to think about your feelings

22. think about how angry you are with yourself 


\section{Emotion Regulation Questionnaire (ERQ)}

\section{Instructions and Items}

We would like to ask you some questions about your emotional life, in particular, how you control (that is, regulate and manage) your emotions. The questions below involve two distinct aspects of your emotional life. One is your emotional experience, or what you feel like inside. The other is your emotional expression, or how you show your emotions in the way you talk, gesture, or behave. Although some of the following questions may seem similar to one another, they differ in important ways. For each item, please answer using the following scale:

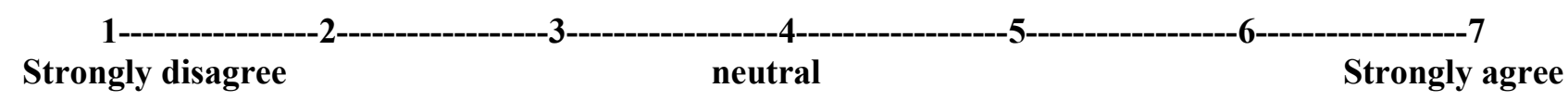

1. When I want to feel more positive emotion (such as joy or amusement), I change what I'm thinking about.

2. I I k keep my emotions to myself.

3. When I want to feel less negative emotion (such as sadness or anger), I change what I'm thinking about.

4. When I am feeling positive emotions, I am careful not to express them.

5. W__ When I'm faced with a stressful situation, I make myself think about it in a way that helps me stay calm.

6. I I control my emotions by not expressing them.

7.___ When I want to feel more positive emotion, I change the way I'm thinking about the situation.

8. I I control my emotions by changing the way I think about the situation I'm in.

9.___ When I am feeling negative emotions, I make sure not to express them.

10. When I want to feel less negative emotion, I change the way I'm thinking about the situation. 


\section{Coping Flexibility Questionnaire (COFLEX)}

People may be faced with changes in their lives: difficult or stressful situations and wishes or goals which cannot be realized as they would prefer. How people cope with these changes differs from one individual to the other. Below you will find statements of how individuals cope with these changes and deal with difficulties. Please indicate to which extent these statements apply to you by ticking the first answer that comes to mind.

When confronted with an important problem

1. I can easily change my approach if necessary

2. I think of different options when a solution is not

$\begin{array}{cccc}\begin{array}{c}\text { Seldom } \\ \text { or } \\ \text { never }\end{array} & \text { Sometimes } & \text { Often } & \begin{array}{c}\text { Almost } \\ \text { always }\end{array} \\ 1 & 2 & 3 & 4 \\ 1 & 2 & 3 & 4\end{array}$

successful

3. I immediately change my approach if a certain

1

2

3

4 approach fails

4. I adjust my strategy as soon as I notice that my

approach fails

5. I think about the effort it will to take to achieve a certain goal

6. I have enough strategies to deal with the problem

7. I am flexible in my approach towards a problem

8. I question myself what is really important to me

9. I have enough different options to quickly solve a problem

10. I usually take some time to think about what I am going to do

11. I question myself whether my approach to the problem is the best solution

12. I find it is a challenge to adapt to changing circumstances

13. I easily think of a different approach that suits the changing situation 


\section{Perseverative Thinking Questionnaire (PTQ)}

In this questionnaire, you will be asked to describe how you typically think about negative experiences or problems. Please read the following statements and rate the extent to which they apply to you when you think about negative experiences or problems.

Never Rarely Sometimes Often Almost

1. The same thoughts keep going through my

$0 \quad 1$

2

3

Always mind again and again.

2. Thoughts intrude into my mind.

3. I can't stop dwelling on them.

4. I think about many problems without solving any of them.

5. I can't do anything else while thinking

$\begin{array}{lllll}0 & 1 & 2 & 3 & 4 \\ 0 & 1 & 2 & 3 & 4 \\ 0 & 1 & 2 & 3 & 4\end{array}$
about my problems.

6. My thoughts repeat themselves.

7. Thoughts come to my mind without me

$\begin{array}{lllll}0 & 1 & 2 & 3 & 4\end{array}$
wanting them to.

8. I get stuck on certain issues and can't move $\quad \begin{array}{llllll}0 & 1 & 2 & 3 & 4\end{array}$ on.

9. I keep asking myself questions without finding an answer.

10. My thoughts prevent me from focusing on other things.

11. I keep thinking about the same issue all the time.

12. Thoughts just pop into my mind.

13. I feel driven to continue dwelling on the

1

$\begin{array}{lll}2 & 3 & 4\end{array}$
same issue.

14. My thoughts are not much help to me.

15. My thoughts take up all my attention.

$\begin{array}{lllll}0 & 1 & 2 & 3 & 4 \\ 0 & 1 & 2 & 3 & 4\end{array}$




\section{Cognitive Flexibility Inventory (CFI)}

Please use the scale below to indicate the extent to which you agree or disagree with the following statements.

Strongly

Disagree

Disagree Disagree Neutral

1
2
3

4

Somewhat Agree Agree Strongly 5

I am good at "sizing up" situations.

I have a hard time making decisions when faced

with difficult situations.

I consider multiple options before making a decision.

$\begin{array}{lllllll}1 & 2 & 3 & 4 & 5 & 6 & 7\end{array}$

When I encounter difficult situations, I feel like I am losing control.

$\begin{array}{lllllll}1 & 2 & 3 & 4 & 5 & 6 & 7\end{array}$

I like to look at difficult situations from many

different angles.

$\begin{array}{lllllll}1 & 2 & 3 & 4 & 5 & 6 & 7\end{array}$

I seek additional information not immediately available before attributing causes to behavior.

$\begin{array}{lllllll}1 & 2 & 3 & 4 & 5 & 6 & 7\end{array}$

When encountering difficult situations, I become so stressed that I can not think of a way to resolve $\begin{array}{lllllll}1 & 2 & 3 & 4 & 5 & 6 & 7\end{array}$ the situation.

I try to think about things from another person's point of view.

$\begin{array}{lllllll}1 & 2 & 3 & 4 & 5 & 6 & 7\end{array}$

I find it troublesome that there are so many

different

$\begin{array}{lllllll}1 & 2 & 3 & 4 & 5 & 6 & 7\end{array}$

ways to deal with difficult situations.

I am good at putting myself in others' shoes.

When I encounter difficult situations, I just don't know what to do.

$\begin{array}{lllllll}1 & 2 & 3 & 4 & 5 & 6 & 7\end{array}$

$\begin{array}{lllllll}1 & 2 & 3 & 4 & 5 & 6 & 7\end{array}$


It is important to look at difficult situations from

many angles.

$\begin{array}{lllllll}1 & 2 & 3 & 4 & 5 & 6 & 7\end{array}$

When in difficult situations, I consider multiple

options before deciding how to behave.

$\begin{array}{lllllll}1 & 2 & 3 & 4 & 5 & 6 & 7\end{array}$

I often look at a situation from different

viewpoints.

$\begin{array}{lllllll}1 & 2 & 3 & 4 & 5 & 6 & 7\end{array}$

I am capable of overcoming the difficulties in life that I face.

$\begin{array}{lllllll}1 & 2 & 3 & 4 & 5 & 6 & 7\end{array}$

I consider all the available facts and information

when attributing causes to behavior.

$\begin{array}{lllllll}1 & 2 & 3 & 4 & 5 & 6 & 7\end{array}$

I feel I have no power to change things in difficult situations.

$\begin{array}{lllllll}1 & 2 & 3 & 4 & 5 & 6 & 7\end{array}$

When I encounter difficult situations, I stop and

try to think of several ways to resolve it.

$\begin{array}{lllllll}1 & 2 & 3 & 4 & 5 & 6 & 7\end{array}$

I can think of more than one way to resolve a

difficult situation I'm confronted with.

$\begin{array}{lllllll}1 & 2 & 3 & 4 & 5 & 6 & 7\end{array}$

I consider multiple options before responding to difficult situations.

$\begin{array}{lllllll}1 & 2 & 3 & 4 & 5 & 6 & 7\end{array}$




\section{Cognitive Style Questionnaire - Short Form (CSQ-SF)}

Please try vividly to imagine yourself in each of the situations that follow. Picture each situation as clearly as you can as if the events were happening to you right now. Place yourself in each situation and decide what you feel would have caused that situation if it actually happened to you. Although the events may have many causes, we want you to choose only one - the thing you feel would be the major cause of the situation if it actually happened to you.

It is important to remember that there are no right or wrong answers to the questions. The important thing is to answer the questions in a way that corresponds to what you would think and feel if the situations occurred in your life. 


\section{Imagine you are getting along badly with your parents}

Think carefully about the reason for you getting along badly with your parents, then answer the questions below

Strongly

Agree

1. Getting along badly with my parents is caused by other people or circumstances*

2. The reason I get on badly with my parents causes problems in all areas of my life

3. My parents and I will start afresh in the future and forget about the reason for getting along badly*

4. Getting along badly with my parents will lead to other negative things $\square$ happening to me

5. Getting along badly with my parents means there is something wrong $\square$ with me as a person

6. It is my fault if I am getting along badly with my parents

7. The reason I get on badly with my parents does not stop me from enjoying other things*

8. The reason for getting along badly will stop me from getting along $\square$ well with my parents in the future

9. Getting along badly with my parents does not say anything about me as a person*
Strongly

Disagree

. 


\section{Imagine your class reacts negatively to an important talk you have to give as part of your coursework}

Think carefully about the reason for the class reacting negatively to your talk, then answer the questions below

Strongly

Agree

1. It is not my fault that people reacted negatively*

2. The reason people reacted negatively to my talk will cause failures in all areas of my life

3. The reason people reacted negatively to this talk means that others will react negatively to talks I give in the future

4. People reacting negatively to my talk will not lead to other negative $\square$ things happening to me*

5. The fact that people reacted negatively to this talk says nothing about me as a person*

6. The reason for the negative reaction was not caused by other people $\square$ or circumstances

7. The reason that caused people to react negatively applies only to this talk*

8. Talks I give in the future will not be affected by the reason people reacted badly to this talk*

9. The fact that people reacted negatively to this talk says a lot about me $\square$ as a person
as person 
3. Imagine that during the first year of working in the career of your choice, you receive a negative evaluation of your job performance

Think carefully about the reason for you getting a negative evaluation, then answer the questions below

Strongly

Agree

1. Other people or circumstances did not cause me to get a negative evaluation

2. The reason I got a negative evaluation applies only to this judgement $\square$ about my job performance*

3. My job evaluations in the future will be affected by the same reason $\square$ that caused this negative evaluation

4. This negative evaluation will lead to other negative things happening to me

5. This negative evaluation means there is something wrong with me as a person

6. It is my fault that I got this negative evaluation

7. The reason for getting this negative evaluation will not cause failures in all areas of my life*

8. The reason for this negative evaluation will not impact on my future $\square$ job evaluations*

9. The fact that I got a negative evaluation says nothing about me as a person*

\section{Undecide Disagree}

Strongly

Disagree 


\section{Imagine you go to a party and people are not interested in you}

Think carefully about the reason for people not being interested in you, then answer the questions below

\section{Strongly \\ Agree}

1. It is not my fault that people are not interested in $\mathrm{me}^{*}$

2. The reason for people not being interested in me applies only to this party*

3. If I go to a party like this in the future, things will be different and people will be interested in me*

4. People not being interested in me at this party will not lead to other $\square$ negative things happening to $\mathrm{me}^{*}$

5. People not being interested in me at this party does not mean there is something wrong with me as a person*

6. The reason for people not being interested in me was not caused by $\square$ other people or events at the party

7. The reason for people not being interested in me at this party will cause problems in all areas of my life

8. The reason people weren't interested in me at this party will cause people at parties in the future not to be interested in me

9. People not being interested in me at this party says a lot about me as a person
Agree

Undecided Disagree

Strongly

Disagree 


\section{Imagine you really want to be in an intimate, romantic relationship, but you aren't}

Think carefully about the reason for you not being in a relationship, then answer the questions below

Strongly

Agree

1. Other people or circumstances have caused me not to be in an intimate, romantic relationship*

2. The reason that causes me not to be in a relationship applies only to intimate, romantic relationships*

3. The reason that causes me not to be in a relationship will have no impact on my future relationships*

4. Not being in an intimate, romantic relationship will lead to other negative things happening to me

5. Not being in an intimate, romantic relationship means there is something wrong with me as a person

6. It is my fault if I am not in an intimate, romantic relationship

7. The reason that causes me not to be in a relationship leads to problems in all areas of my life

8. The reason that causes me not to be in a relationship will mean I will not have an intimate, romantic relationship in the future

9. Not being in intimate, romantic relationship says nothing about me $\square$ as a person* 
6. Imagine that in an important class, you can't complete all the work that your teacher expects of you

Think carefully about the reason for you failing to complete the work, then answer the questions below

Strongly

Agree
Undecided
Strongly

Disagree

1 It is not my fault that I failed to complete the work*

2 The reason for not completing the work will cause problems in al areas of my life

3 The reason I failed to complete the work will cause similar failure in $\square$ completing work in classes in the future

4 Failing to complete the work will not lead to other negative things happening to $\mathrm{me}^{*}$

5 Failing to complete this work says a lot about me as a person

6 Other people or events caused me not to complete the work*

7 The reason for failing to complete the work applies only to this class* $\square$

8 The reason I failed to complete this work will not impact on whether $\square$ I complete work in future classes*

9. Not completing this work says nothing about me as a person* 
7. Imagine a person you'd really like to develop a close friendship does not want to be friends

with you

Think carefully about the reason for the person not wanting to be friends with you, then answer the questions below

Strongly

Agree

1 Other people or circumstances have caused this person not to want $\square$ to be my friend*

2 The reason that causes this person not to want to be my friend applies only to this friendship*

3 The reason that causes this person not to want to be my friend will always prevent this person from becoming my friend

4 This person not wanting to be my friend will lead to other negative things happening to me

5 This person not wanting to be my friend means there is something wrong with me as a person

6 It is my fault that this person does not want to be my friend

7 The reason that causes this person not to want to be my friend leads to problems in all areas of my life

8 This person may feel differently about the reason that caused them not $\square$ to want to be my friend, and become my friend in the future*

9 This person not wanting to be my friend says nothing about me as a person*

\section{Agree Undecided Disagree}

Strongly

Disagree 


\section{Imagine you are unhappy}

Think carefully about the reason for you being unhappy, then answer the questions below

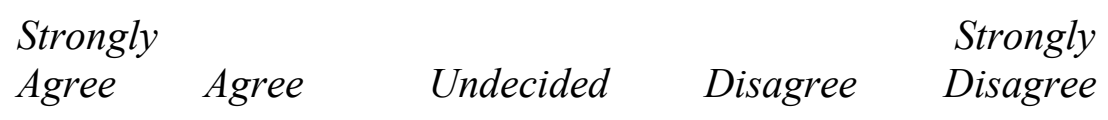

1. My unhappiness is caused by other people or circumstances*

2. The reason for my unhappiness affects only my mood*

3. The reason for my unhappiness will always cause me to be unhappy $\square$

4. Being unhappy will not lead to other negative things happening to me* $\square$

5. Being unhappy does not mean there's something wrong with me as a $\square$ person*

6. It is my fault that I am unhappy

7. The reason I'm unhappy causes problems in all areas of my life

8. The reason why I'm unhappy will go away and never cause me to be $\square$ unhappy in the future*

9. Being unhappy says a lot about me as a person 


\section{Dysfunctional Attitudes Scale - Short Form (DAS-SF)}

The sentences below describe people's attitudes. Circle the number which best describes how much each sentence describes your attitude. Your answer should describe the way you think most of the time.

\begin{tabular}{|c|c|c|}
\hline $\begin{array}{l}\text { Totally } \\
\text { Agree }\end{array}$ & Agree & Disagree \\
\hline
\end{tabular}

1. If I don't set the highest standards for rate person.

2. My value as a person depends greatly

3. People will probably think less of me if I

4. I am nothing if a person I love doesn't love me.

5. If other people know what you are really like, they will think less of you.

6. If I fail at my work, then I am a failure as a person.

7. My happiness depends more on other people than it does me.

8. I cannot be happy unless most people I know admire me.

9. It is best to give up your own interests 


\section{Survey of Coping Profiles Endorsed (SCOPE)}

The purpose of this questionnaire is to find out how people deal with their problems or the stresses in their lives. The following are activities that you may have done. After each activity, please indicate the extent to which you would use this as a way of dealing with problems or stresses in recent weeks.

Ordinarily, in recent weeks have you

Never Seldom Sometimes Often Almost always

1. accepted that there was nothing you could do to change your situation?

$\begin{array}{llllllll}\text { 2. } & \text { tried to just take whatever came your way? } & 0 & 1 & 2 & 3 & 4\end{array}$

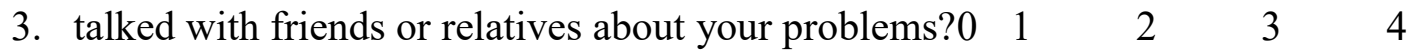

4. tried to do things which you typically enjoy? $\quad \begin{array}{lllllll}0 & 1 & 2 & 3 & 4\end{array}$

5. sought out information that would help you $\quad \begin{array}{lllllll}0 & 1 & 2 & 3 & 4\end{array}$ resolve your problems?

$\begin{array}{llllllll}\text { 6. blamed others for creating your problems } & & 0 & 1 & 2 & 3 & 4\end{array}$ or making them worse?

7. sought the advice of others to resolve your problems? $0 \begin{array}{lllll}1 & 2 & 3 & 4\end{array}$

$\begin{array}{llllllll}\text { 8. blamed yourself for your problems? } & 0 & 1 & 2 & 3 & 4\end{array}$

$\begin{array}{llllllll}\text { 9. } \text { exercised? } & 0 & 1 & 2 & 3 & 4\end{array}$

10. fantasized or thought about unreal things $\quad \begin{array}{lllllll}0 & 1 & 2 & 3 & 4\end{array}$

(eg., the perfect revenge, or winning a million dollars) to feel better?

$\begin{array}{lllllll}\text { 11. been very emotional compared to your usual self? } & 0 & 1 & 2 & 3 & 4\end{array}$

12. gone over your problems in your mind $\quad \begin{array}{llllll}0 & 1 & 2 & 3 & 4\end{array}$ over and over again?

13. asked others for help?

$\begin{array}{lllll}0 & 1 & 2 & 3 & 4\end{array}$

14. thought about your problems a lot? $\quad \begin{array}{llllll}0 & 1 & 2 & 3 & 4\end{array}$

15. became involved in recreation or pleasure activities? $0 \begin{array}{llllll}0 & 1 & 2 & 3 & 4\end{array}$

16. worried about your problems a lot? $\quad \begin{array}{llllll}0 & 1 & 2 & 3 & 4\end{array}$

17. tried to keep your mind off things that $\quad \begin{array}{llllll}0 & 1 & 2 & 3 & 4\end{array}$ 
are upsetting you?

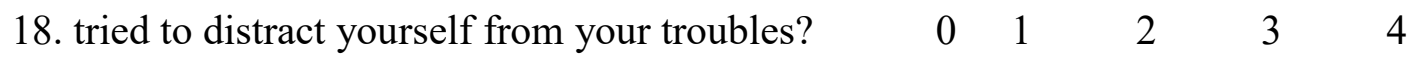

$\begin{array}{lllllll}\text { 19. avoided thinking about your problems? } & 0 & 1 & 2 & 3 & 4\end{array}$

$\begin{array}{llllllll}\text { 20. made plans to overcome your problems? } & 0 & 1 & 2 & 3 & 4\end{array}$

$\begin{array}{lllllll}\text { 21. told jokes about your situation? } & 0 & 1 & 2 & 3 & 4\end{array}$

22. thought a lot about who is responsible for $\quad \begin{array}{lllllll}0 & 1 & 2 & 3 & 4\end{array}$ your problems (besides yourself)?

23. shared humorous stories etc. to cheer yourself $\quad \begin{array}{llllllll}0 & 1 & 2 & 3 & 4\end{array}$ and others up?

24. told yourself that other people have dealt with $\quad \begin{array}{lllllll}0 & 1 & 2 & 3 & 4\end{array}$ problems such as yours?

25. thought a lot about how you have brought $\quad \begin{array}{llllll}0 & 1 & 2 & 3 & 4\end{array}$ your problems on yourself?

$\begin{array}{llllllll}26 . & \text { decided to wait and see how things turn out? } & 0 & 1 & 2 & 3 & 4\end{array}$

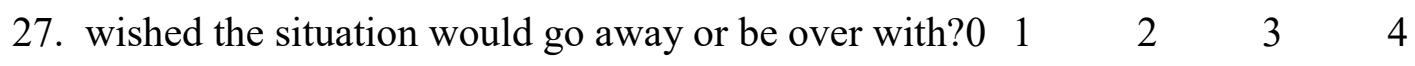

28. decided that your current problems are a $\quad \begin{array}{llllllll}0 & & & 1 & 2 & 3 & 4\end{array}$

result of your own past actions?

29. gone shopping? $\quad \begin{array}{lllllll}0 & 1 & 2 & 3 & 4\end{array}$

30. asserted yourself and taken positive action on $\quad \begin{array}{llllllll}0 & 1 & 2 & 3 & 4\end{array}$

problems that are getting you down?

31. sought reassurance and moral support from others? $\begin{array}{lllllll}0 & 1 & 2 & 3 & 4\end{array}$

$\begin{array}{llllllll}\text { 32. resigned yourself to your problems? } & 0 & 1 & 2 & 3 & 4\end{array}$

33. thought about how your problems have been $\quad \begin{array}{llllllll}0 & 1 & 2 & 3 & 4\end{array}$ caused by other people?

$\begin{array}{lllllllll}\text { 34. daydreamed about how things may turn out? } & 0 & 1 & 2 & 3 & 4\end{array}$

$\begin{array}{lllllllll}\text { 35. been very emotional in how you react, } & & 0 & 1 & 2 & 3 & 4\end{array}$ even to little things?

36. decided that you can grow and learn through $\quad \begin{array}{llllllll}0 & 1 & 2 & 3 & 4\end{array}$ your problems? 
$\begin{array}{llllllll}\text { 37. told yourself that other people have problems } & & 0 & 1 & 2 & 3 & 4\end{array}$ like your own?

$\begin{array}{lllllll}\text { 38. wished I was a stronger person or better at dealing } & 0 & 1 & 2 & 3 & 4\end{array}$ with problems?

39. looked for how you can learn something out of $\begin{array}{lllllll}0 & 1 & 2 & 3 & 4\end{array}$ your bad situation?

40. asked for God's guidance?

41. kept your feelings bottled up inside?

42. found yourself crying more than usual?

43. tried to act as if you were not upset?

44. prayed for help?

45. gone out?

46. held in your feelings?

47. tried to act as if you weren't feeling bad?

48. taken steps to overcome your problems?

49. made humorous comments or wise cracks?

50 . told others that you were depressed or

$\begin{array}{lllll}0 & 1 & 2 & 3 & 4 \\ 0 & 1 & 2 & 3 & 4 \\ 0 & 1 & 2 & 3 & 4 \\ 0 & 1 & 2 & 3 & 4 \\ 0 & 1 & 2 & 3 & 4 \\ 0 & 1 & 2 & 3 & 4 \\ 0 & 1 & 2 & 3 & 4 \\ 0 & 1 & 2 & 3 & 4 \\ 0 & 1 & 2 & 3 & 4 \\ 0 & 1 & 2 & 3 & 4 \\ 0 & 1 & 2 & 3 & 4\end{array}$
emotionally upset? 